\title{
CLIMATOLOGICAL DATA FOR THE RICE-GROWING AREAS \\ ALONG THE NORTH COAST OF PUERTO RICO
}

By

Angel Román-Más and Bruce Green

U.S. GEOLOGICAL SURVEY

OPEN-FILE DATA REPORT 86-53

Prepared in cooperation with the PUERTO RICO DEPARTMENT OF AGRICULTURE

San Juan, Puerto Rico 1987 


\title{
DEPARTMENT OF THE INTERIOR
}

\author{
DONALD PAUL HODEL, Secretary
}

\author{
U.S. GEOLOGICAL SURVEY \\ Dallas L. Peck, Director
}

For additional information

write to:

Chief, Carlbbean District, WRD

U.S. Geologlcal Survey

GPO BOX 4424

San Juan, Puerto Rlco 00936
Coples of this report can be purchased from:

U.S. Geological Survey Books and Open-FIle Reports Federal Center, Bldg. 41 Box 25425

Denver, Colorado 80225 


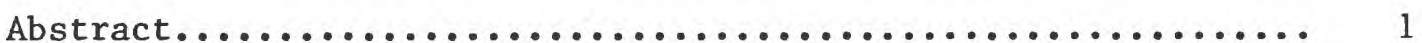

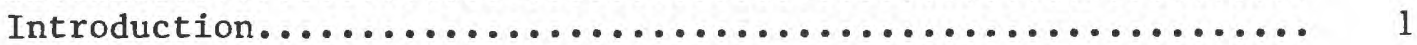

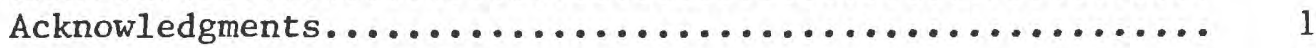

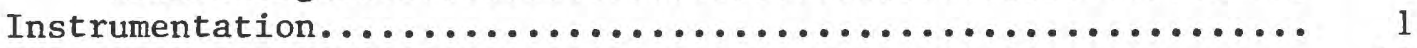

Significance of weather data for rice cultivation........... 11

References.................................. 90

\section{ILLUSTRATIONS}

Figure 1. Map showing location of rice-growing areas along the north coast of Puerto Rico and weather

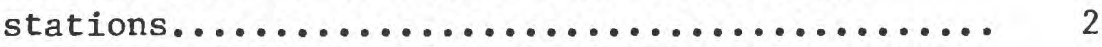

2. Graph showing daily rainfall at the Vega Baja, Manat $i$ and Arecibo weather stations........... 3

3. Graph showing daily maximum, minimum, and mean temperatures at the Vega Baja, Manati, and Arecibo weather stations.................... 4

4. Graph showing daily wind velocity at the Vega Baja, Manati, and Arecibo weather stations......... 5

5. Graph showing daily pan evaporation at the Vega Baja, Manati, and Arecibo weather stations..... 6

6. Photographs showing two 8-inch nonrecording rainfall gages (copper and plastic and models).. 7

7. Photographs showing a submersible minimummaximum thermometer, Model E8-11 (A) and a Weather Service medium-sized shelter (B)....... 9

8. Photograph showing a 5-digit odometer type, 3cup anemometer....................... 10

9. Photographs showing a U.S. Weather Service class A land pan (top) and a hook-gage in a stilling well (bottom).................. 12

10 Photograph showing a micrometer hook-gage........ 13

\section{TABLES}

Table 1. Weather data and statistical analysis for the Vega Baja weather station.................14-43

2. Weather data and statistical analysis for the Manati weather station.................44-68

3. Weather data and statistical analysis for the Arecibo weather station.................69-89 


\section{CONVERSION TABLE}

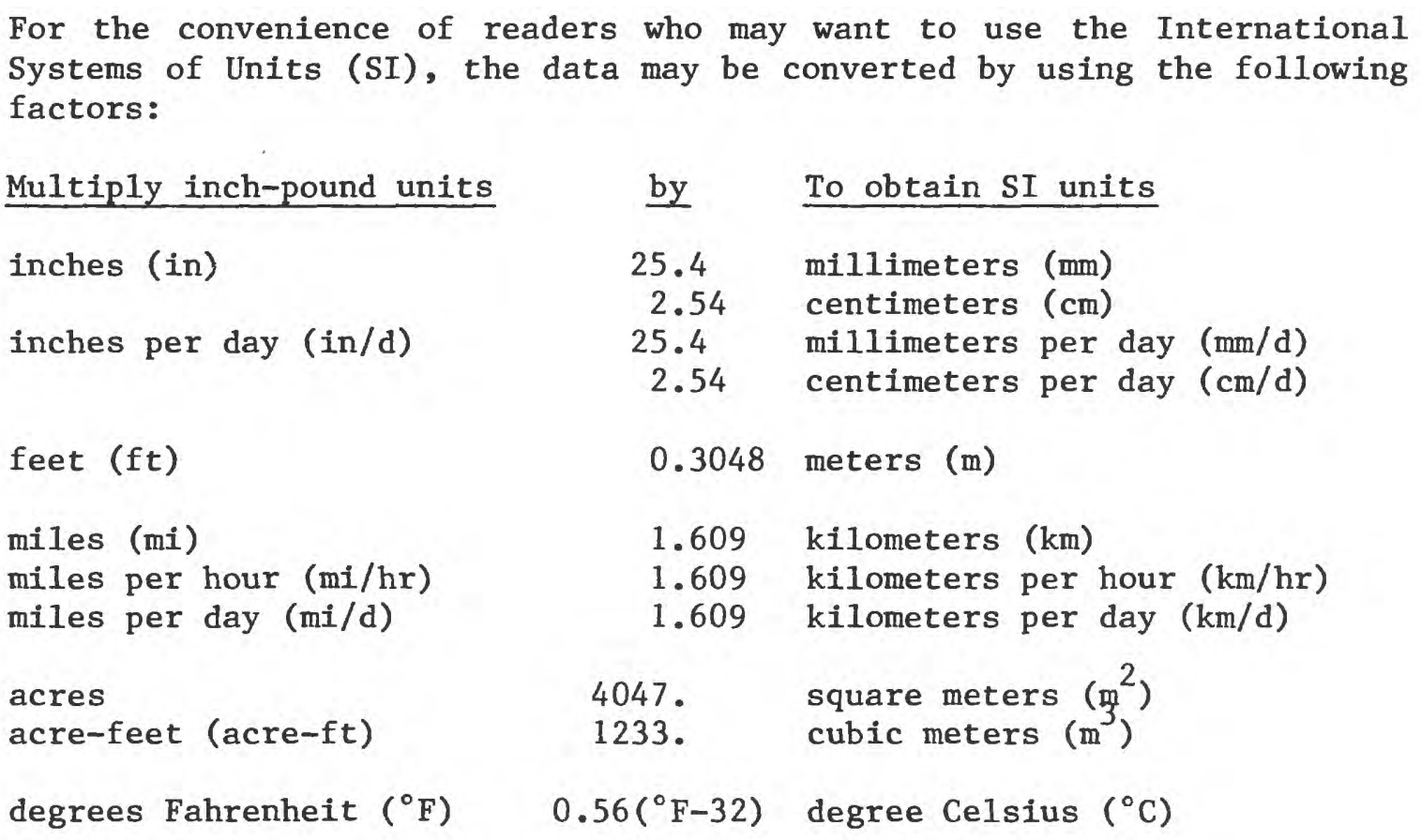

\section{Specific Combinations}

1 Acre-ft $=226.2 \mathrm{gal} / \mathrm{min}$, during one day

$1 \mathrm{ft}^{3} / \mathrm{s}=448.8 \mathrm{gal} / \mathrm{min}$

$1 \mathrm{ft}^{3} / \mathrm{s}=0.65 \mathrm{Mga} 1 / \mathrm{d}$ 


\title{
CLIMATOLOGICAL DATA FOR \\ THE RICE-GROWING AREAS ALONG \\ THE NORTH COAST OF PUERTO RICO
}

\author{
By \\ Angel J. Román-Más and Bruce Green
}

\begin{abstract}
Rainfal1, temperature, wind velocity and pan evaporation data were collected from May 1983 to September 1985, in the rice-growing areas of Vega Baja, Manatí, and Arecibo in northern Puerto Rico. Daily values and statistics including mean, standard deviation, extremes, and totals for each month of record were compiled. Descriptions of equipment installation and operation, data processing, and significance of climatological data for rice cultivation are presented.
\end{abstract}

\section{INTRODUCTION}

In 1983, the Caribbean District office of the U.S. Geological Survey, Water Resources Division (USGS) in cooperation with the Puerto Rico Department of Agriculture (PRDOA), began an investigation to determine the quantity of water required for irrigation in the rice-growing areas of Arecibo, Manatí, and Vega Baja (fig. 1). As part of this investigation, a weather station was located at each area (fig. 2, 3, and 4). Rainfall, temperature, wind velocity, and pan evaporation data were collected daily from May 1983 to September 1985. Climatological data was used to define the prevailing weather conditions during the crop cycles measured.

This report summarizes the rainfall, temperature, wind velocity, and pan evaporation data collected at each weather station (fig. 2, 3, 4, and 5). Means and extreme values, standard deviations, and totals for the month ( $t a b l e s ~ 1,2$, and 3) are included.

\section{Acknowledgments}

The authors gratefully acknow1edge the assistance provided by hydrologic technicians José Merced and Ana Sánchez in the instrument installation as well as in the collection and processing of the data.

\section{INSTRUMENTATION}

Each station was equipped with a rain gage, a maximum-minimum thermometer, an evaporation pan, and an anemometer. Equipment installation and operation as well as data collection by the USGS technical staff was in accordance with standard National Oceanographic and Atmospheric Administration (NOAA) methods and procedures criteria. 


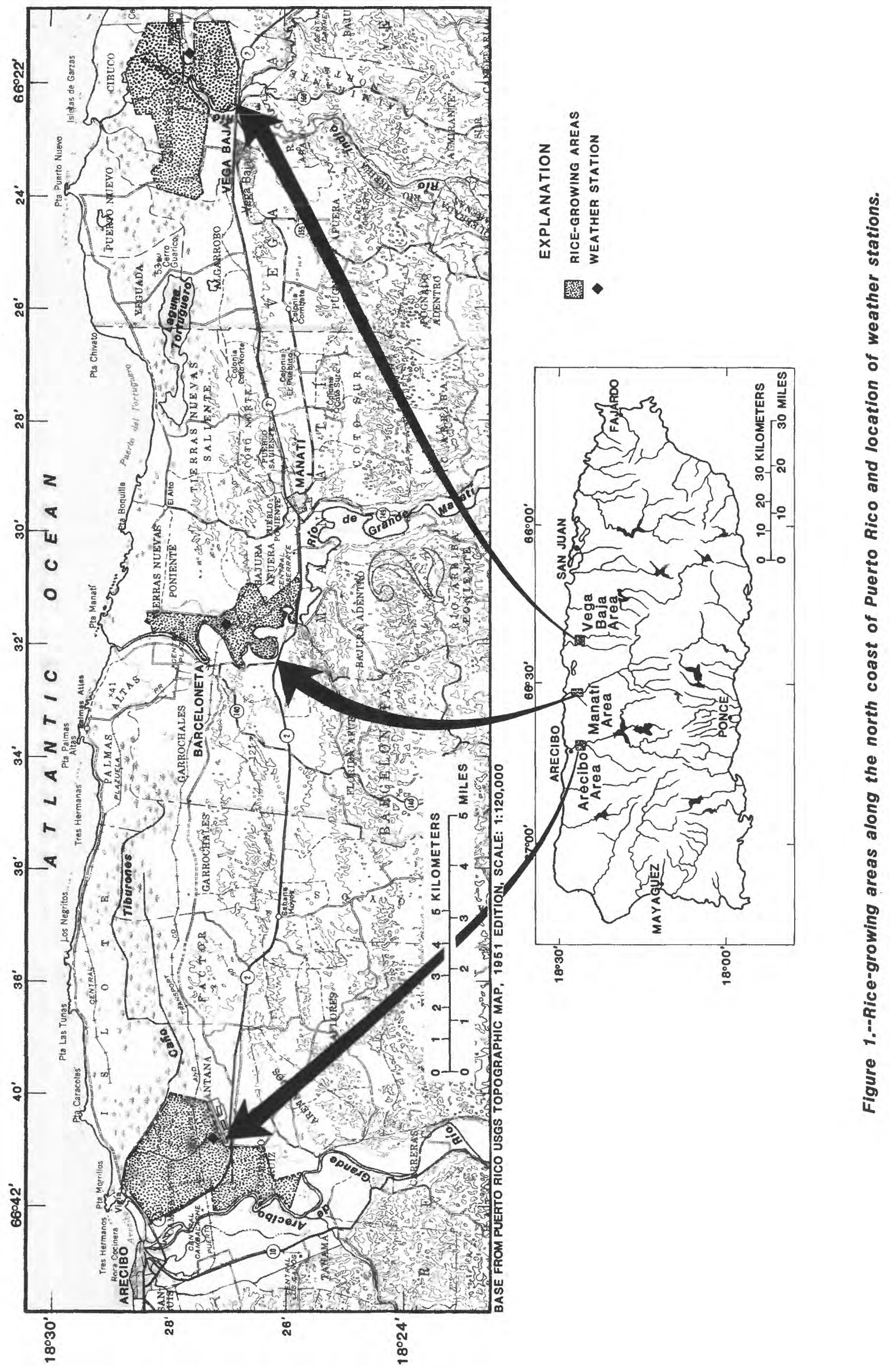



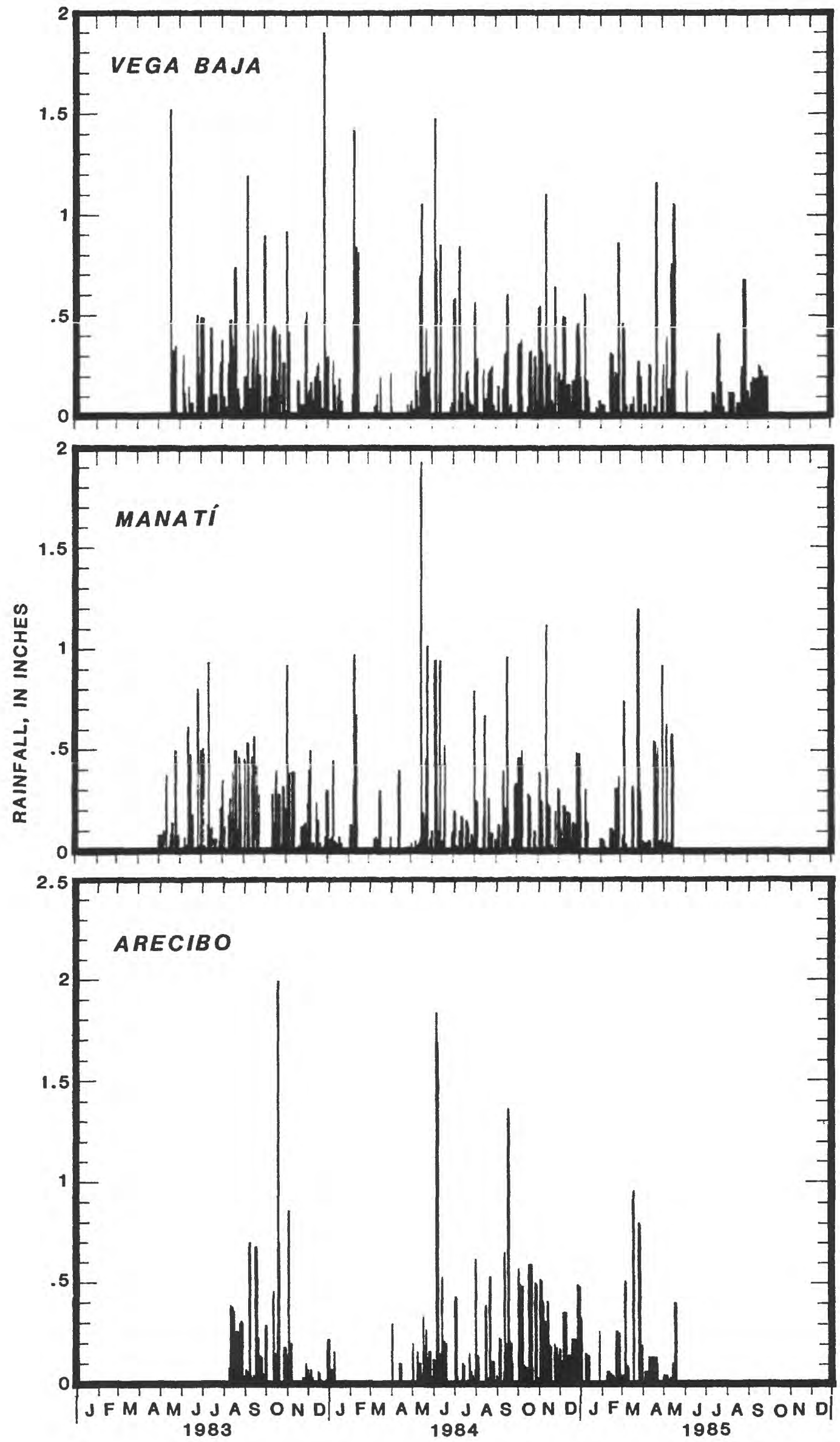

Figure 2.--Daily rainfall at the Vega Baja, Manat, and Arecibo weather stations. 

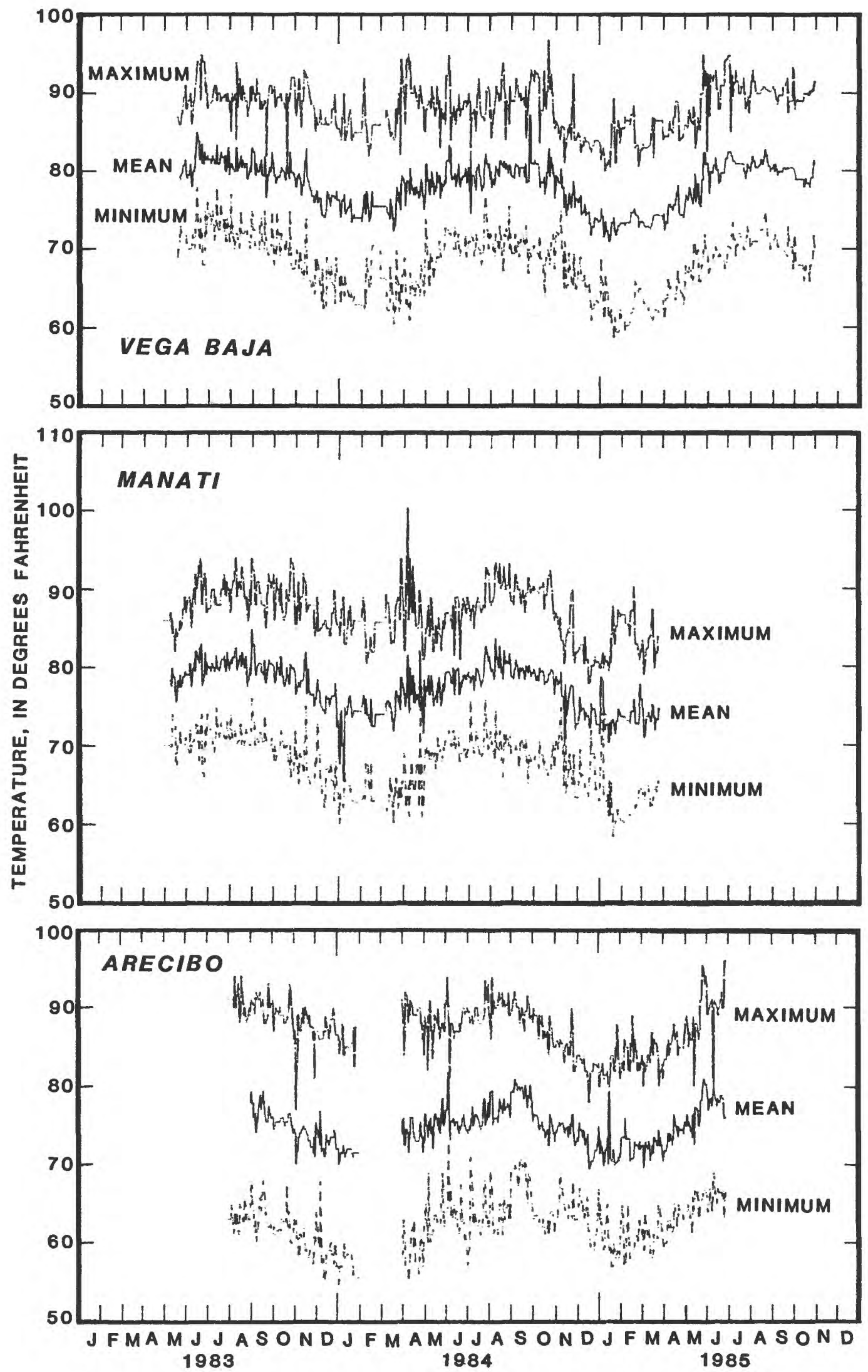

Figure 3.--Daily maximum, minimum, and mean temperatures at the Vega Baja, Manati, and Arecibo weather statlons. 

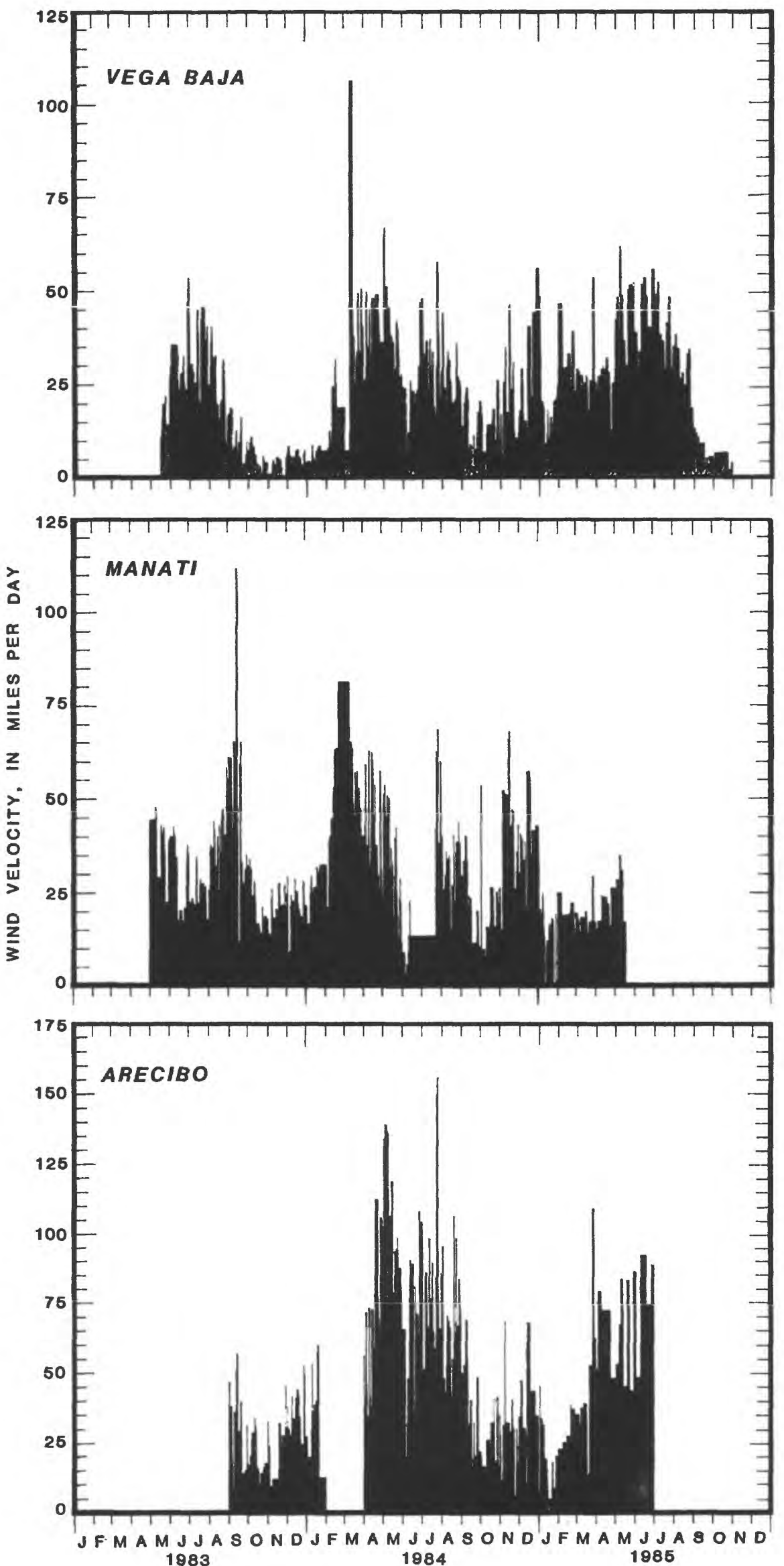

Figure 4.--Daily wind velocity at the Vega Baja, Manat, and Arecibo weather stations. 

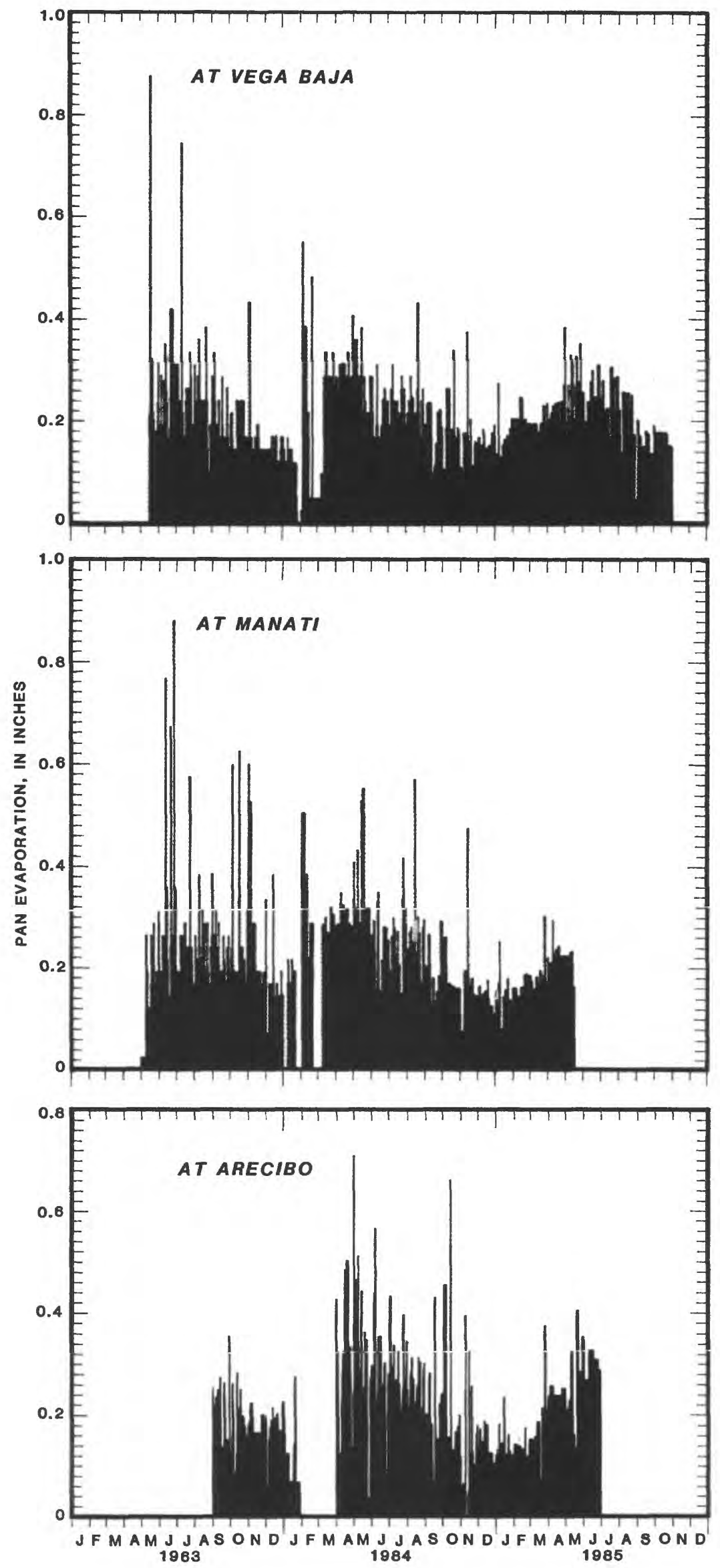

Flgure 5.--Daily pan evaporation at the Vega Baja, Manati, and Arecibo weather stations. 


\section{INSTRUMENTATION--Continued}

Rainfall was measured with an 8-inch non-recording gage made of either plastic or metal. The gage consists of an overflow can, measuring tub, rainfall funnel, measuring stick, and support ( $\mathrm{fig}$. 6). The top of the funnel is exactly 8-inches inside diameter. The funnel empties the rainfall into a cylindrical measuring tub. To facilitate measurement of rainfall to hundredths of an inch, the measuring tube has a cross-section area that is one-tenth the top area of the funnel. The scale of the

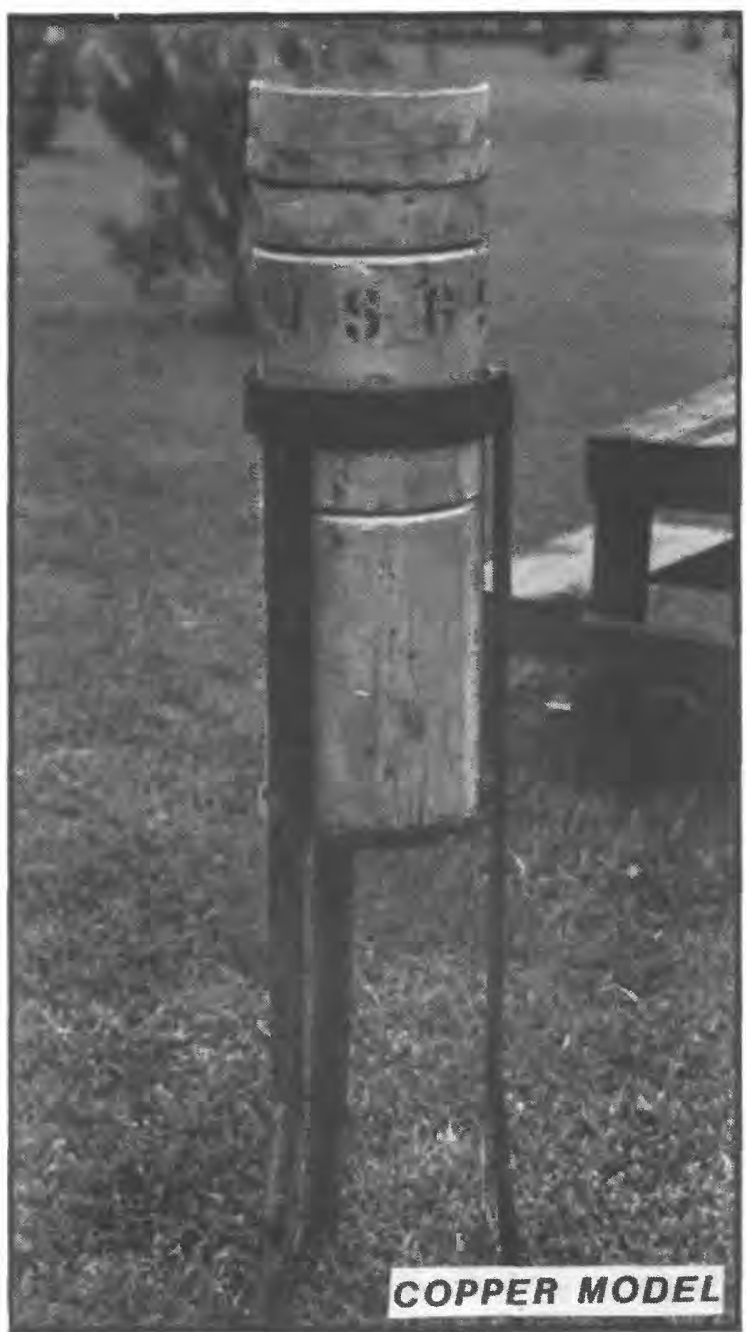

measuring stick used with the tube is expanded 10 times, and since the scale is graduated to hundredths of an inch, the correct rainfall depth of water in the tube is read directly to hundredths from the stick. The measuring tube is 20 inches in height and holds exactly 2.00 inches of rainfall. Any additional rainfall will overflow into the outer can. Water from the can is poured into an empty tube for direct measurement with the stick. The measuring tube of the plastic model has been graduated to hundredths of an inch to read directly without using the stick.

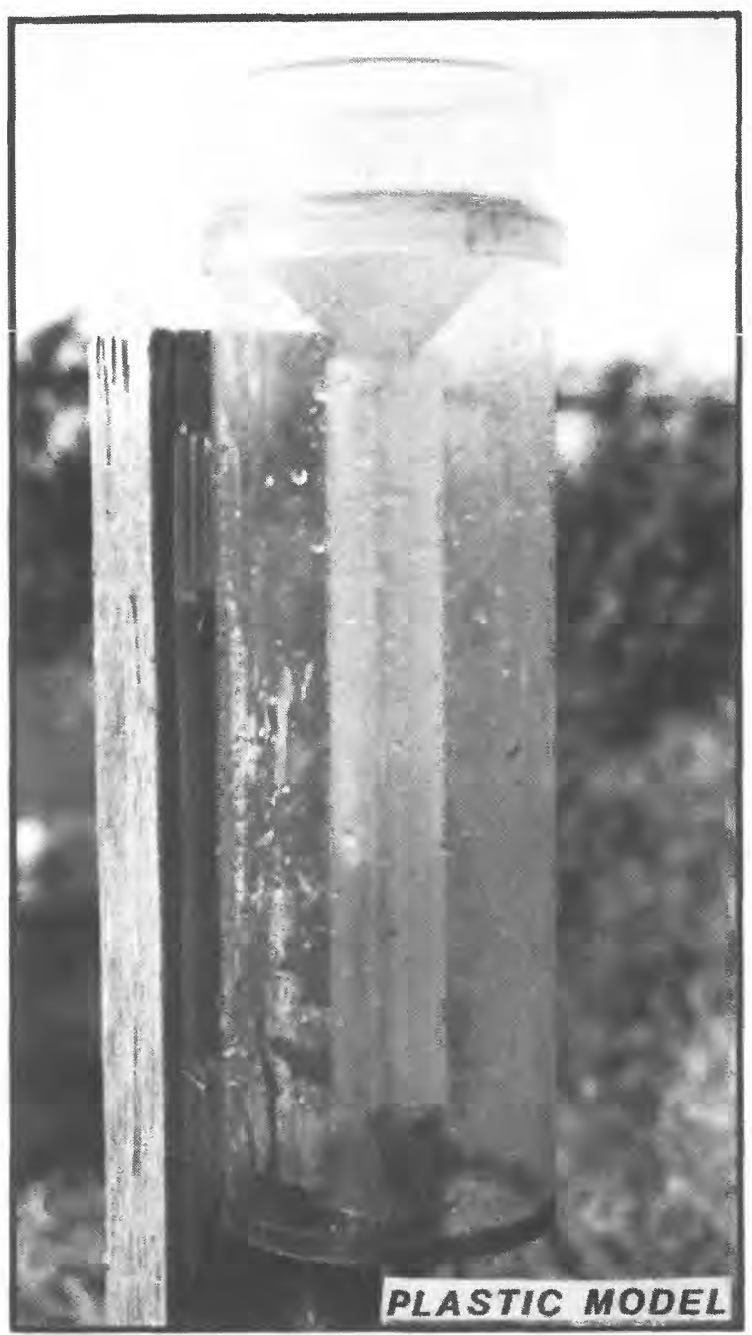

Figure 6.--Two 8-inch nonrecording rainfall gages (copper and plastic models). 


\section{INSTRUMENTATION--Continued}

The Weather Measure Model E8-11 (Use of trade names by the USGS does not imply endoresement of the products depicted), Submersible Minimum-Maximum Thermometer was used to measure minimum and maximum temperatures. It consists of a plastic backing with a glass $U$ tube containing two metal indices - one to indicate minimum temperature and one to indicate maximum temperature. In addition, the glass U tube contains a liquid filling and a mercury column. Two temperature scales are permanently marked on the U tube, one for low temperatures and the other for high temperatures (fig. 7). In operation, as the temperature changes, the liquid filling located above the mercury columns in both legs of the $U$ tube, expands or contracts depending upon whether or not the temperature is rising or falling. If the temperature is rising, the mercury column in the right-hand leg of the $U$ tube rises. If the temperature is falling, the mercury column in the left-hand leg of the $U$ tube rises. As the mercury column rises, in either the lefthand or right-hand leg of the $U$ tube, it pushes the metal index up higher in the tube. When the mercury column falls, in either leg of the $U$ tube, the metal index remains in place as an indication of the highest or lowest temperature which was reached during the observation period. The bottom edge of the metal index is always read in order to obtain the maximum or minimum temperature.

At the completion of an observation, the metal indices are reset with a small magnet until their lower edges are in contact with the mercury column. The thermometers were mounted on a vertical post near the center of a U.S. National Weather Service (USNWS) medium-sized type shelter. The shelter has louvered sides with a double top designed to protect the thermometers from precipitation, condensation, and radiation. The louvered construction permits air to circulate freely through the shelter.

A five-digit odometer type, 3-cup anemometer was used for measuring wind movement. It is mounted on the wooden evaporation pan support in order to measure the wind movement over the pan. The five digits appearing in the window of the meter (fig. 8) indicate the total wind movement to tenths of a mile for any total from zero to 10,000 miles. The right-hand digit indicates tenths of a mile. Ten thousand miles is indicated as five zeros (00000) on the meter and coincides with zero miles for the succeeding 10,000-mile cycle of operation. If 24-hour wind movement computation is desired, the previous reading has to be subtracted from the current day reading.

The U.S. National Weather Service "Class A" land Pan was used to measure evaporation. This instrument is used at all official USNWS and cooperative stations in the United States. It is made of unpainted galvanized iron, $4 \mathrm{ft}$ in diameter, and 10 inches deep (fig. 9). The bottom, supported on a wooden frame, is raised 6-inches above the ground surface. The water surface, maintained between 2- and 3-inches below the rim of the pan, is measured daily with a hook-gage in a stilling well. The daily evaporation is computed as the difference between observed water levels, correlated for any precipitation measured in an adjacent or standard rain gage. 

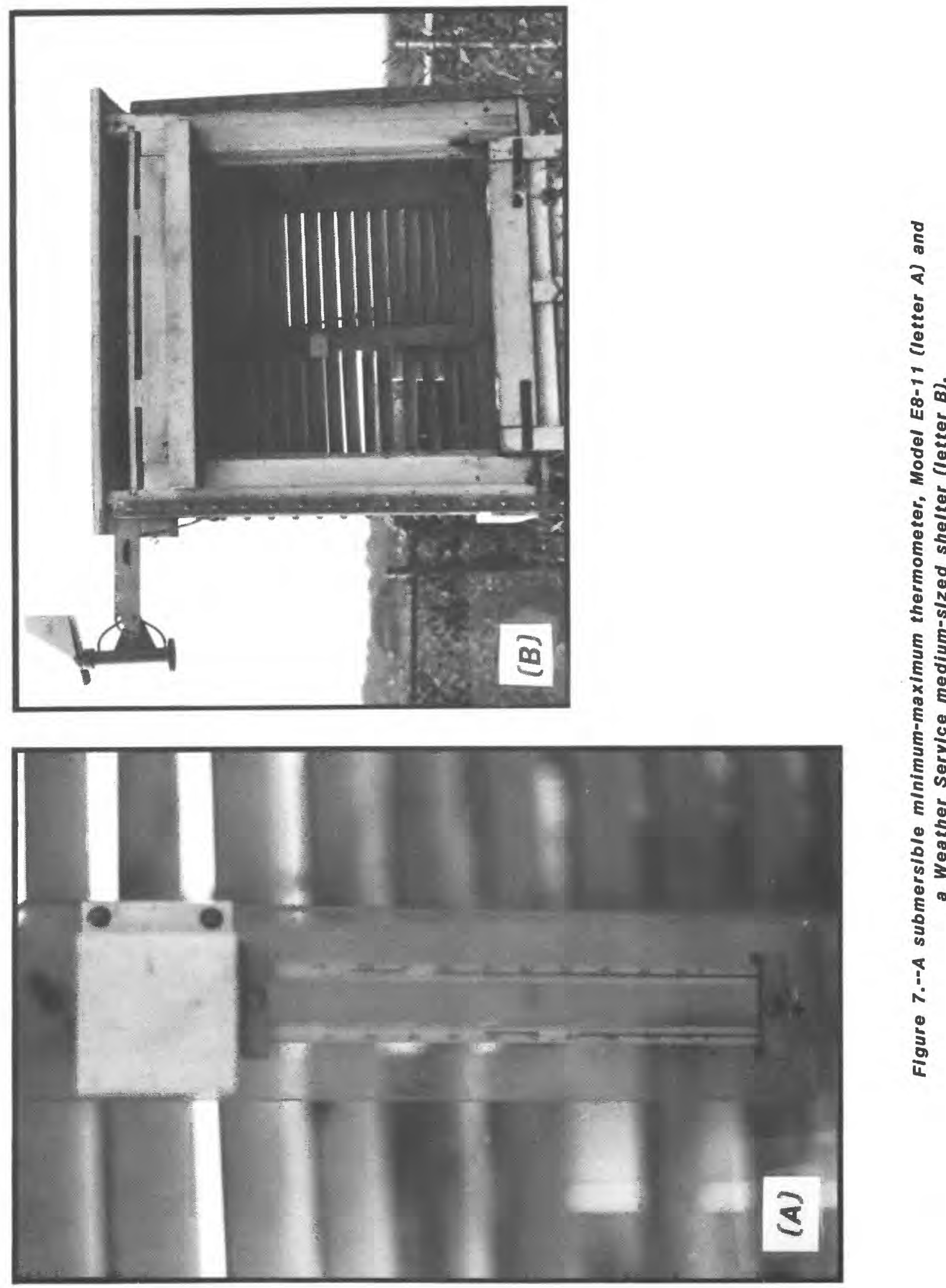


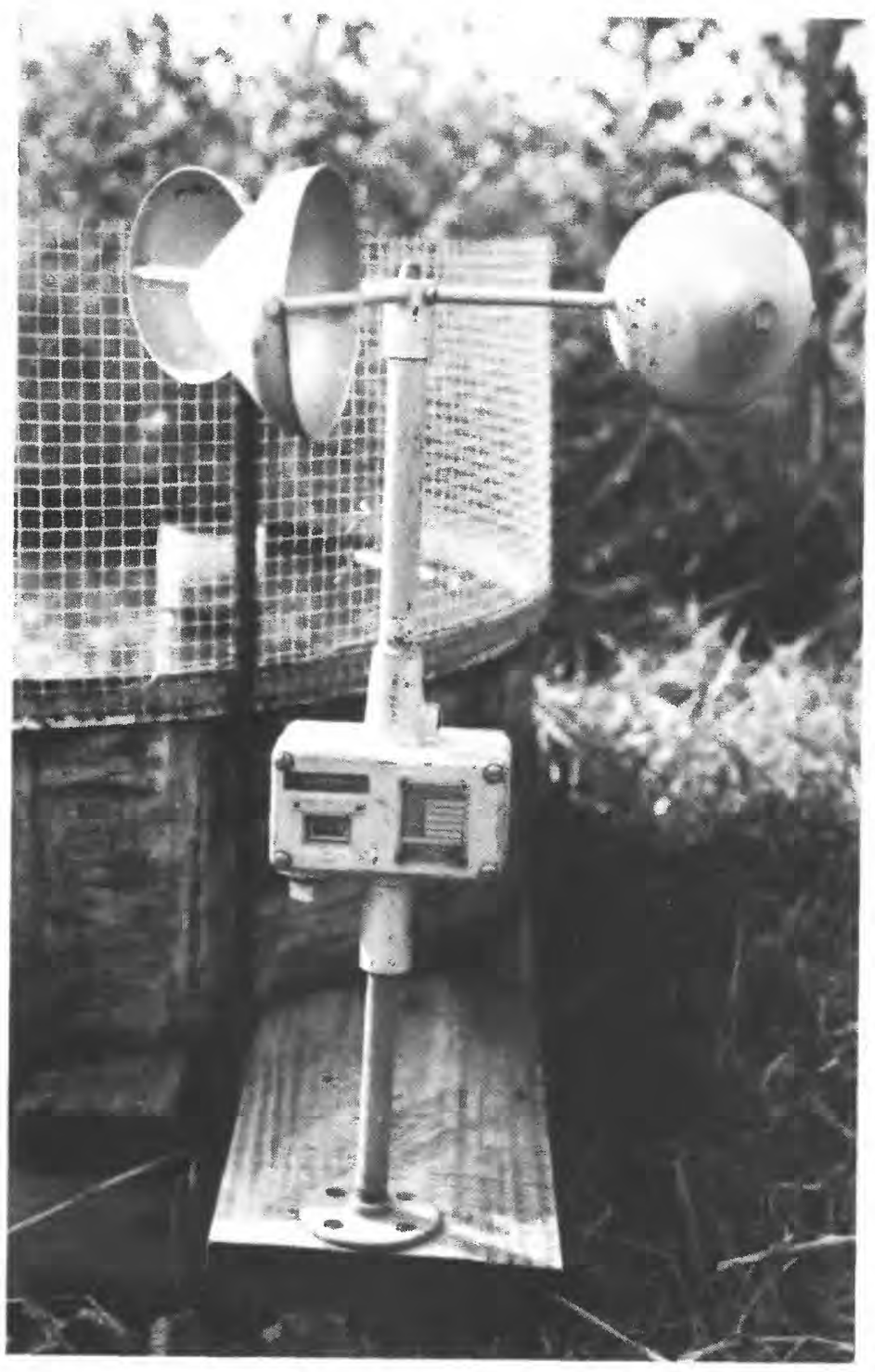

Flgure 8.--A 5-digit odometer type, 3-cup anemometer. 


\section{INSTRUMENTATION--Continued}

A micrometer hook-gage (fig. 10) was used to measure *water level in the evaporation pan. The gage consists of a hook in the end of a sten that is graduated to tenths of inches over a range of several inches. The stem is constructed with double threads throughout its range of adjustment. The threads have a pitch of one-tenth of an inch. A three-legged spider and adjusting-nut assembly supports the hook and provides for adjustment of the height of the hook when the gage is installed on the stilling we11. The adjusting nut is threaded to screw on the stem of the hook. After assembly, the gage is placed on the stilling well with the three legs of the spider resting on the top rim of the well and the hook centered in the well. The adjusting nut is free to turn within the spider so that it may be used conveniently to adjust the height of the hook. The relative height of the hook in the well is indicated by the scales of the gage.

\section{SIGNIFICANCE OF CLIMATOLOGICAL DATA FOR RICE CULTIVATION}

\footnotetext{
Climate is a critical factor in defining the suitability of specific areas for rice cultivation. As an example, rice grows well when partially submerged in water. In comparison to other grains, it requires large amounts of water for maximum yield (weeds control). In addition to accessibility to large supplies of water
}

(rainfall, river, lakes, aquifers) areas suitable for rice cultivation should have warm temperatures, low winds, and low evaporation rates (Vicente-Chandler, 1977).

The total amount of water required by a crop for its normal growth is defined as the water lost by evapotranspiration and infiltration, plus the amount of water required by the irrigation technique utilized (in the case of rice continuous flooding). Experiments conducted by Silva and VicenteChandler (1982, p. 185) and RománMás (1985, p.16) indicated that about 2.90 acre-ft/acre of the 4 acre-ft/acre (73 percent) of water required for rice irrigation is evapotranspired. The use of pan evaporation values to estimated actual evapotranspiration is a widely accepted technique. A series of correlations utilizing temperature, wind velocity, and pan evaporation data might provide a theoretical model by which evapotranspiration can be estimated for other areas.

Irrigation required is the quantity of water other than from precipitation that has to be supplied for crop production. For a rice crop it can be determined by subtracting the precipitation during a period of 120 consecutive days (which is approximately the duration of a crop cycle for rice) from the water required. Data collected indicate that rainfall provides from 0.28 acre-ft/acre ( 7 percent) to 2.43 (61 percent) of the total water required. This assumes an efficient use of the rainfa1l, which may or may not be possible. 

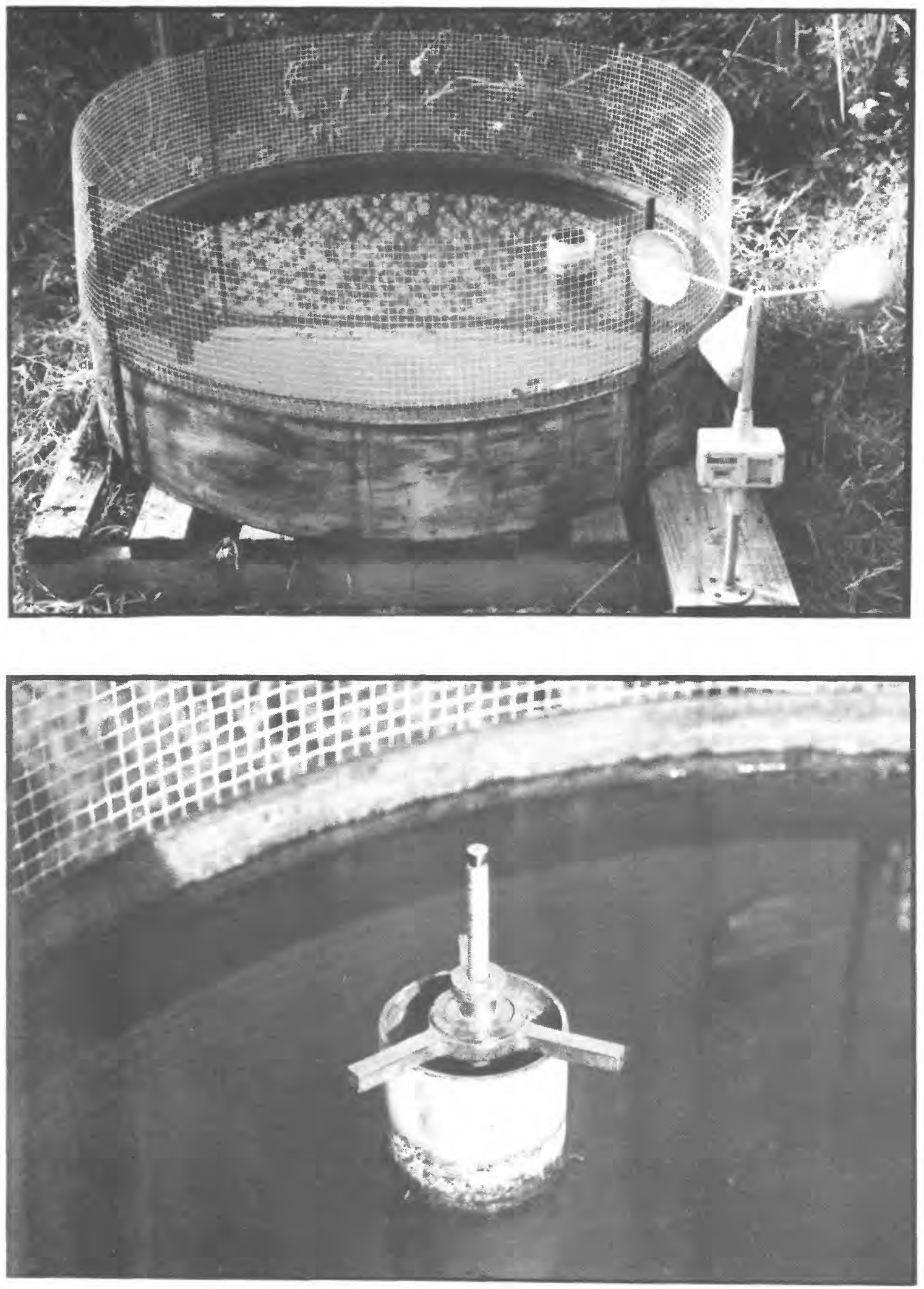

Figure 9.--U.S. Weather service class A land pan (Top) and a hook-gage in a stilling well (Bottom). 


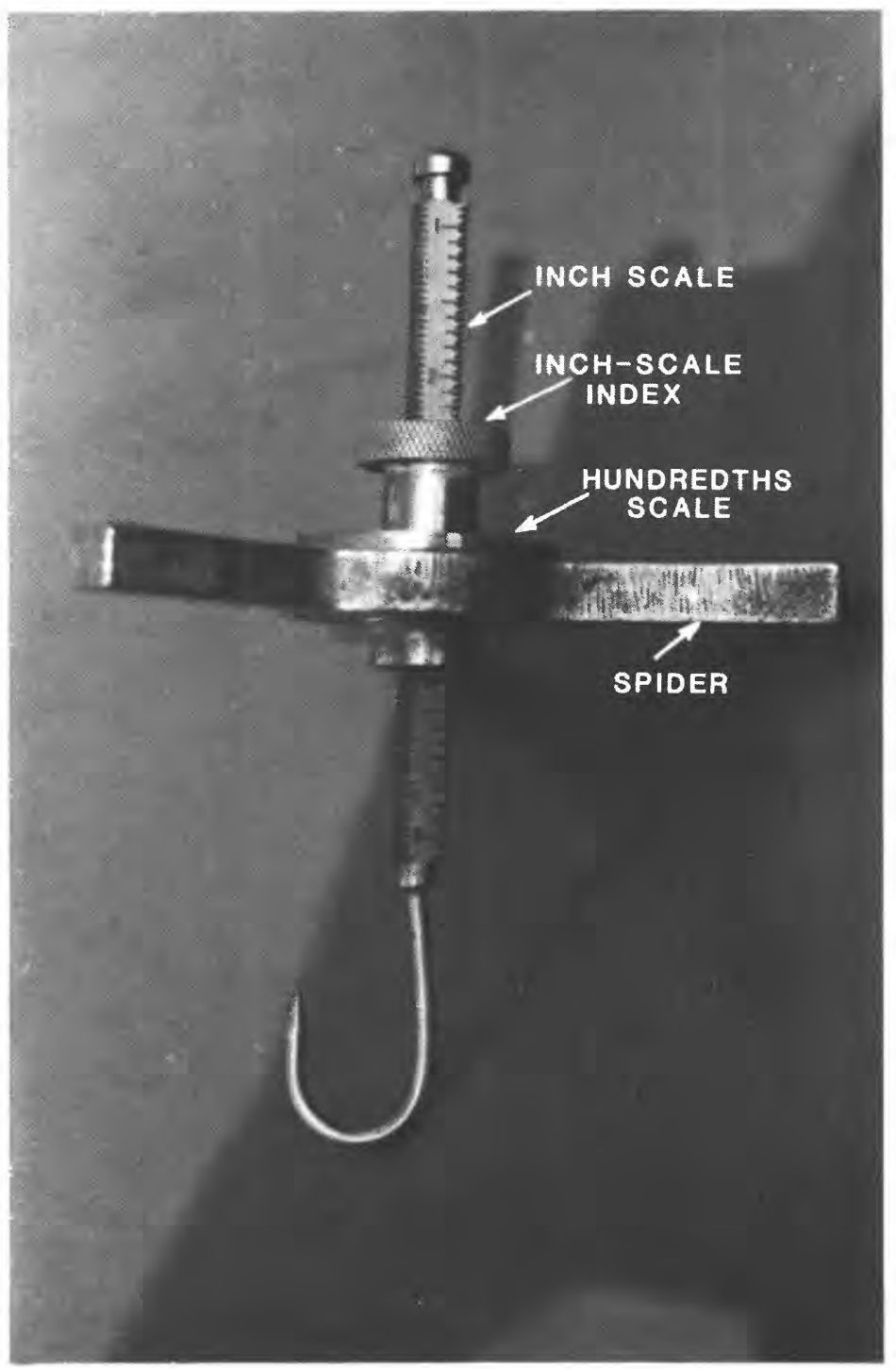

Figure 10.--A micrometer hook-gage. 
Table 1. Weather data and statistical analyses for the Vega Baja weather station May 1983

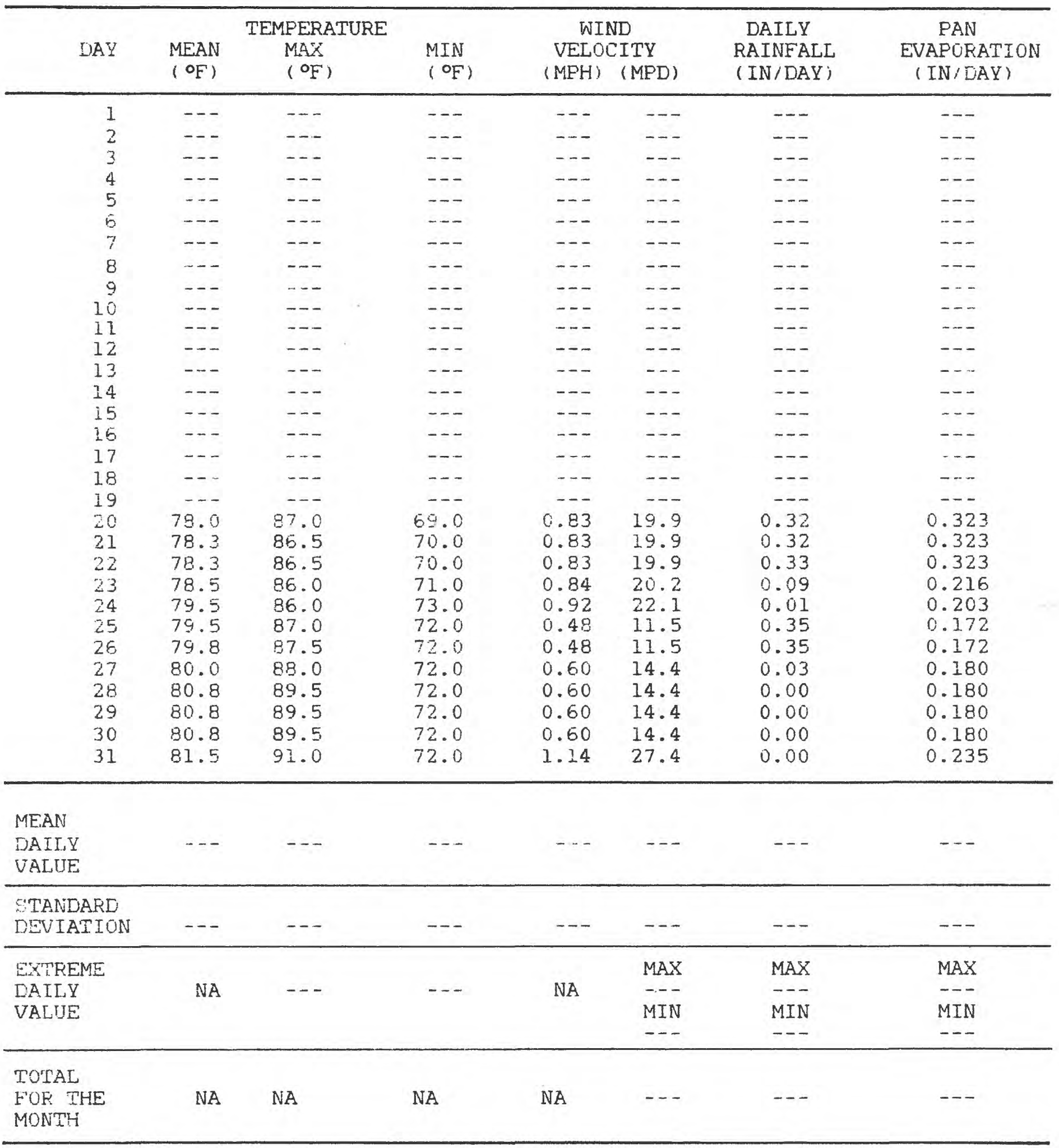


Table 1. (Continued)

VEGA BAJA JUNE 1983

\begin{tabular}{|c|c|c|c|c|c|c|c|}
\hline DAY & $\begin{array}{l}\text { MEAN } \\
\left({ }^{\circ} \mathrm{F}\right)\end{array}$ & $\begin{array}{c}\text { EMPERA: } \\
\text { MAX } \\
\left({ }^{\circ} \mathrm{F}\right)\end{array}$ & $\begin{array}{l}\text { MIN } \\
\left({ }^{\circ} F\right)\end{array}$ & $\begin{array}{l}\text { WII } \\
\text { VELO } \\
\text { (MPH) }\end{array}$ & $\begin{array}{l}\text { ID } \\
\text { (MPY) } \\
\text { (MPD) }\end{array}$ & $\begin{array}{c}\text { DAILY } \\
\text { RAINFALL } \\
\text { (IN/DAY) }\end{array}$ & $\begin{array}{c}\text { PAN } \\
\text { EVAPORATION } \\
(\text { IN/DAY })\end{array}$ \\
\hline $\begin{array}{l}1 \\
2 \\
3 \\
4 \\
5 \\
6 \\
7 \\
8 \\
9 \\
10 \\
11 \\
12 \\
13 \\
14 \\
15 \\
16 \\
17 \\
18 \\
19 \\
20 \\
21 \\
22 \\
23 \\
24 \\
25 \\
26 \\
27 \\
28 \\
29 \\
30\end{array}$ & $\begin{array}{l}80.0 \\
79.0 \\
79.5 \\
79.5 \\
79.5 \\
80.0 \\
81.0 \\
79.0 \\
78.0 \\
79.5 \\
80.0 \\
80.0 \\
80.5 \\
82.0 \\
83.0 \\
85.0 \\
84.5 \\
83.8 \\
83.8 \\
82.0 \\
81.5 \\
82.5 \\
83.5 \\
81.0 \\
81.0 \\
81.0 \\
81.0 \\
82.5 \\
81.5 \\
81.5\end{array}$ & $\begin{array}{l}88.0 \\
88.0 \\
89.0 \\
89.0 \\
89.0 \\
70.0 \\
90.0 \\
88.0 \\
86.0 \\
87.0 \\
89.0 \\
89.0 \\
91.0 \\
91.0 \\
92.0 \\
93.0 \\
94.0 \\
94.0 \\
94.0 \\
94.0 \\
89.0 \\
94.0 \\
95.0 \\
94.0 \\
94.0 \\
94.0 \\
94.0 \\
90.0 \\
91.0 \\
90.0\end{array}$ & $\begin{array}{l}72.0 \\
70.0 \\
70.0 \\
70.0 \\
70.0 \\
70.0 \\
72.0 \\
70.0 \\
70.0 \\
72.0 \\
71.0 \\
71.0 \\
70.0 \\
73.0 \\
74.0 \\
78.0 \\
75.0 \\
72.5 \\
72.5 \\
70.0 \\
74.0 \\
71.0 \\
72.0 \\
68.0 \\
68.0 \\
68.0 \\
68.0 \\
75.0 \\
72.0 \\
73.0\end{array}$ & $\begin{array}{l}1.20 \\
1.50 \\
1.50 \\
1.50 \\
1.50 \\
1.48 \\
1.49 \\
1.49 \\
1.49 \\
1.39 \\
1.39 \\
1.39 \\
1.01 \\
0.89 \\
0.99 \\
0.92 \\
1.17 \\
1.17 \\
1.17 \\
1.17 \\
1.37 \\
1.18 \\
0.99 \\
0.99 \\
0.99 \\
0.99 \\
0.78 \\
1.96 \\
2.23 \\
1.85\end{array}$ & $\begin{array}{l}28.8 \\
35.9 \\
35.9 \\
35.9 \\
35.9 \\
35.5 \\
35.8 \\
35.8 \\
35.8 \\
35.8 \\
35.8 \\
33.3 \\
24.1 \\
21.5 \\
24.0 \\
22.1 \\
28.1 \\
28.1 \\
28.1 \\
28.1 \\
32.9 \\
28.4 \\
23.8 \\
23.8 \\
23.8 \\
3.8 \\
18.8 \\
47.0 \\
53.5 \\
44.4\end{array}$ & $\begin{array}{l}0.00 \\
0.00 \\
0.00 \\
0.00 \\
0.00 \\
0.31 \\
0.08 \\
0.12 \\
0.05 \\
0.00 \\
0.00 \\
0.00 \\
0.00 \\
0.15 \\
0.03 \\
0.00 \\
0.07 \\
0.07 \\
0.07 \\
0.01 \\
0.00 \\
0.00 \\
0.00 \\
0.00 \\
0.05 \\
0.50 \\
0.30 \\
0.00 \\
0.18 \\
0.21\end{array}$ & $\begin{array}{l}0.314 \\
0.192 \\
0.192 \\
0.192 \\
0.192 \\
0.291 \\
0.188 \\
0.188 \\
0.188 \\
0.280 \\
0.280 \\
0.280 \\
0.229 \\
0.353 \\
0.262 \\
0.236 \\
0.165 \\
0.165 \\
0.165 \\
0.165 \\
0.186 \\
0.112 \\
0.420 \\
0.420 \\
0.420 \\
0.420 \\
0.133 \\
0.163 \\
0.286 \\
0.307\end{array}$ \\
\hline $\begin{array}{l}\text { MEAN } \\
\text { DAILY } \\
\text { VALUE }\end{array}$ & 81.2 & 90.3 & 71.4 & 1.30 & 31.8 & 0.07 & 0.246 \\
\hline $\begin{array}{l}\text { STANDARD } \\
\text { DEVIATION }\end{array}$ & 1.73 & 4.56 & 2.29 & 0.325 & 9.25 & 0.120 & 0.089 \\
\hline $\begin{array}{l}\text { EXTREME } \\
\text { DAILY } \\
\text { VALUE }\end{array}$ & NA & 95.0 & 68.0 & NA & $\begin{array}{l}\text { MAX } \\
53.5 \\
\text { MIN } \\
03.8\end{array}$ & $\begin{array}{l}\text { MAX } \\
0.31 \\
\text { MIN } \\
0.00\end{array}$ & $\begin{array}{l}\text { MAX } \\
0.420 \\
\text { MIN } \\
0.133\end{array}$ \\
\hline $\begin{array}{l}\text { TOTAL } \\
\text { FOR THE } \\
\text { MONTH }\end{array}$ & NA & NA & NA & NA & 953.3 & 2.10 & 7.384 \\
\hline
\end{tabular}


Table 1. (Cont/nued)

VEGA BAJA JULY 1983

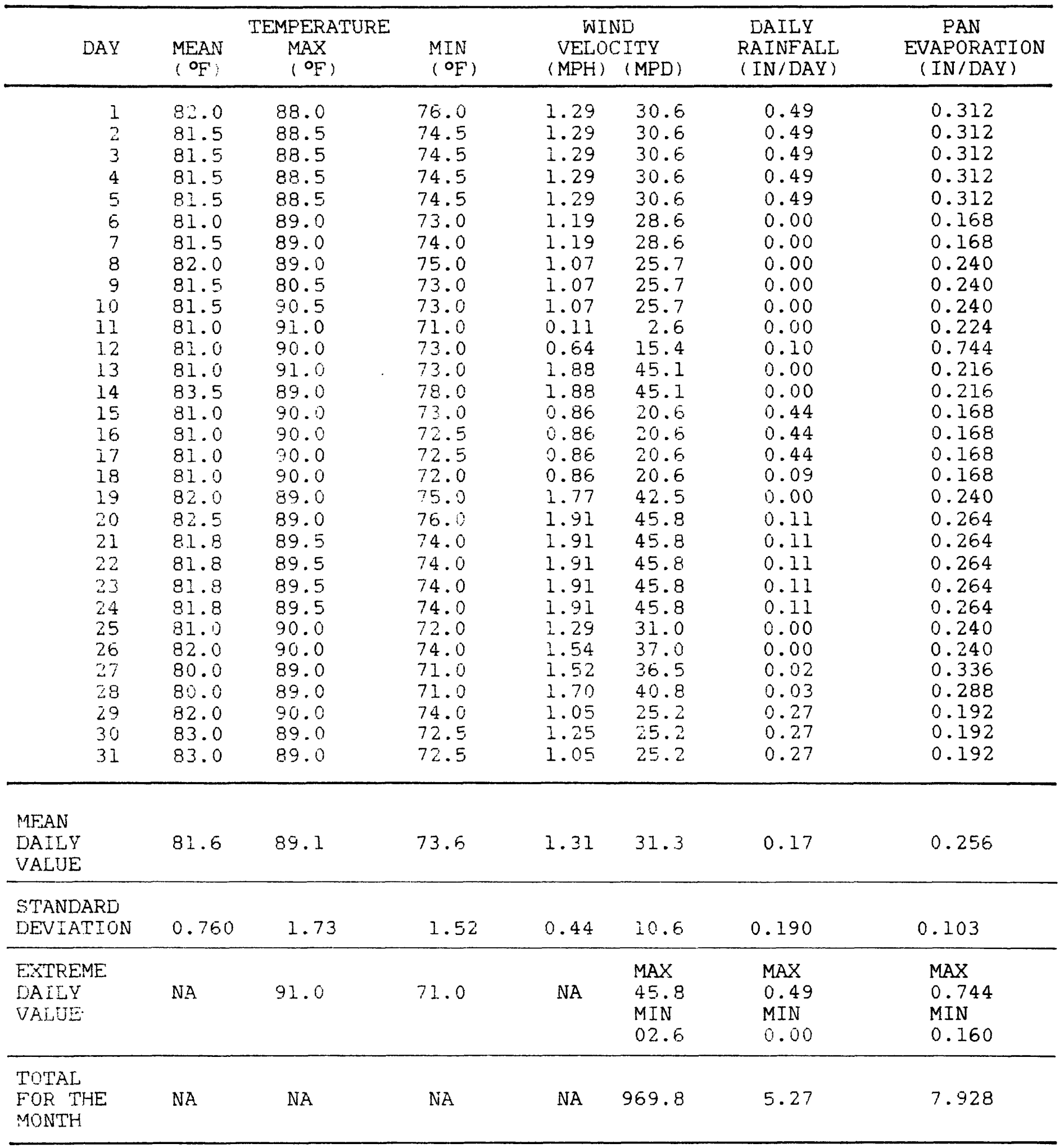


Table 1. (Continued)

VEGA BAJA AUGUST 1983

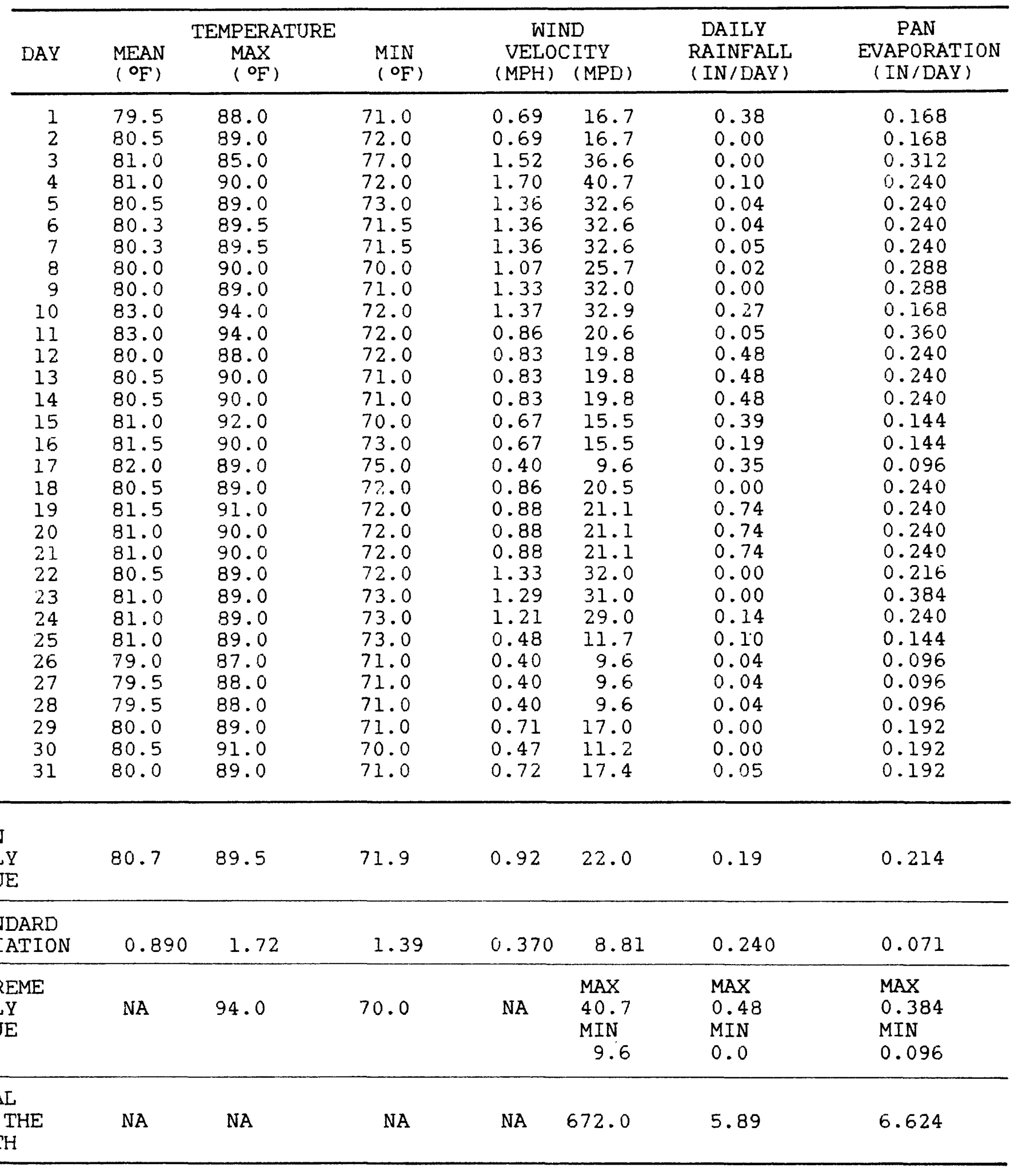


Table 1. (Continued)

VEGA BAJA SEPTEMBER 1983

\begin{tabular}{|c|c|c|c|c|c|c|c|}
\hline DAY & $\begin{array}{l}\text { MEAN } \\
\left(O^{\circ}\right)\end{array}$ & $\begin{array}{l}\text { TEMPERA } \\
\text { MAX } \\
\left({ }^{\circ} \mathrm{F}\right)\end{array}$ & $\begin{array}{l}\text { MIN } \\
(\mathrm{OF})\end{array}$ & $\begin{array}{l}\text { WII } \\
\text { VELOC } \\
(\mathrm{MPH})\end{array}$ & $\begin{array}{l}\text { ND } \\
\text { CITY } \\
\text { (MPD) }\end{array}$ & $\begin{array}{l}\text { DAILY } \\
\text { RAINFALL } \\
\text { (IN/DAY) }\end{array}$ & $\begin{array}{c}\text { PAN } \\
\text { EVAPORATION } \\
(\text { IN/DAY })\end{array}$ \\
\hline $\begin{array}{l}1 \\
2 \\
3 \\
4 \\
5 \\
6 \\
7 \\
8 \\
9 \\
10 \\
11 \\
12 \\
13 \\
14 \\
15 \\
16 \\
17 \\
18 \\
19 \\
20 \\
21 \\
22 \\
23 \\
24 \\
25 \\
26 \\
27 \\
28 \\
29 \\
30\end{array}$ & $\begin{array}{l}83.0 \\
83.0 \\
82.5 \\
82.5 \\
82.5 \\
79.5 \\
79.5 \\
81.0 \\
80.0 \\
80.0 \\
80.0 \\
80.0 \\
80.5 \\
81.0 \\
81.5 \\
79.5 \\
79.5 \\
79.5 \\
79.5 \\
82.0 \\
80.0 \\
76.5 \\
79.0 \\
79.5 \\
79.5 \\
80.0 \\
80.5 \\
80.0 \\
80.5 \\
82.5\end{array}$ & $\begin{array}{l}91.0 \\
91.0 \\
90.0 \\
90.0 \\
90.0 \\
89.0 \\
89.0 \\
92.0 \\
90.0 \\
90.0 \\
90.0 \\
90.0 \\
88.0 \\
88.5 \\
89.0 \\
88.0 \\
88.5 \\
88.5 \\
89.0 \\
89.0 \\
88.0 \\
82.0 \\
88.0 \\
89.5 \\
89.5 \\
91.0 \\
91.0 \\
91.0 \\
91.0 \\
90.0\end{array}$ & $\begin{array}{l}75.0 \\
75.0 \\
72.5 \\
72.5 \\
72.5 \\
70.0 \\
70.0 \\
70.0 \\
70.0 \\
70.0 \\
70.0 \\
70.0 \\
73.0 \\
73.5 \\
74.0 \\
71.0 \\
70.5 \\
70.5 \\
70.0 \\
75.0 \\
72.0 \\
71.0 \\
70.0 \\
69.5 \\
69: 5 \\
69.0 \\
70.0 \\
70.0 \\
70.0 \\
75.0\end{array}$ & $\begin{array}{l}0.73 \\
0.78 \\
0.78 \\
0.78 \\
0.78 \\
0.32 \\
0.12 \\
0.32 \\
0.35 \\
0.35 \\
0.35 \\
0.53 \\
0.29 \\
0.29 \\
0.19 \\
0.40 \\
0.40 \\
0.40 \\
0.68 \\
0.52 \\
0.01 \\
0.01 \\
0.15 \\
0.15 \\
0.15 \\
0.15 \\
0.26 \\
0.26 \\
0.40 \\
0.29\end{array}$ & $\begin{array}{r}17.4 \\
18.8 \\
18.8 \\
18.8 \\
18.8 \\
7.6 \\
3.0 \\
7.7 \\
8.4 \\
8.4 \\
8.4 \\
12.8 \\
6.9 \\
6.9 \\
4.7 \\
9.6 \\
9.6 \\
9.6 \\
16.4 \\
12.5 \\
2.2 \\
2.2 \\
3.7 \\
3.7 \\
3.7 \\
3.7 \\
6.2 \\
6.2 \\
9.6 \\
7.0\end{array}$ & $\begin{array}{l}0.05 \\
0.20 \\
0.20 \\
0.20 \\
0.20 \\
0.15 \\
1.20 \\
0.00 \\
0.14 \\
0.14 \\
0.14 \\
0.02 \\
0.29 \\
0.29 \\
0.43 \\
0.14 \\
0.14 \\
0.14 \\
0.26 \\
0.28 \\
0.46 \\
0.02 \\
0.21 \\
0.21 \\
0.21 \\
0.00 \\
0.00 \\
0.00 \\
0.00 \\
0.09\end{array}$ & $\begin{array}{l}0.192 \\
0.288 \\
0.288 \\
0.288 \\
0.288 \\
0.336 \\
0.240 \\
0.096 \\
0.192 \\
0.192 \\
0.192 \\
0.168 \\
0.240 \\
0.240 \\
0.096 \\
0.168 \\
0.168 \\
0.168 \\
0.288 \\
0.192 \\
0.096 \\
0.120 \\
0.168 \\
0.168 \\
0.168 \\
0.168 \\
0.264 \\
0.264 \\
0.168 \\
0.144\end{array}$ \\
\hline $\begin{array}{l}\text { MEAN } \\
\text { DAILY } \\
\text { VALUE }\end{array}$ & 80.5 & 83.4 & 71.4 & 0.37 & 9.1 & 0.19 & 0.202 \\
\hline $\begin{array}{l}\text { STANDARD } \\
\text { DEVIATION }\end{array}$ & 1.41 & 1.74 & 1.89 & 0.230 & 5.26 & 0.220 & 0.064 \\
\hline $\begin{array}{l}\text { EXTREME } \\
\text { DAILY } \\
\text { VALUE }\end{array}$ & NA & 92.0 & 69.5 & NA & $\begin{array}{l}\text { MAX } \\
18.8 \\
\text { MIN } \\
2.2\end{array}$ & $\begin{array}{l}\text { MAX } \\
1.20 \\
\text { MIN } \\
0.0\end{array}$ & $\begin{array}{l}\text { MAX } \\
0.336 \\
\text { MIN } \\
0.096\end{array}$ \\
\hline $\begin{array}{l}\text { TOTAL } \\
\text { FOR THE } \\
\text { MONTH }\end{array}$ & NA & NA & NA & NA & 273.3 & 5.70 & 6.000 \\
\hline
\end{tabular}


Table 1. (Cont/nued)

VEGA BAJA OCTOBER 1983

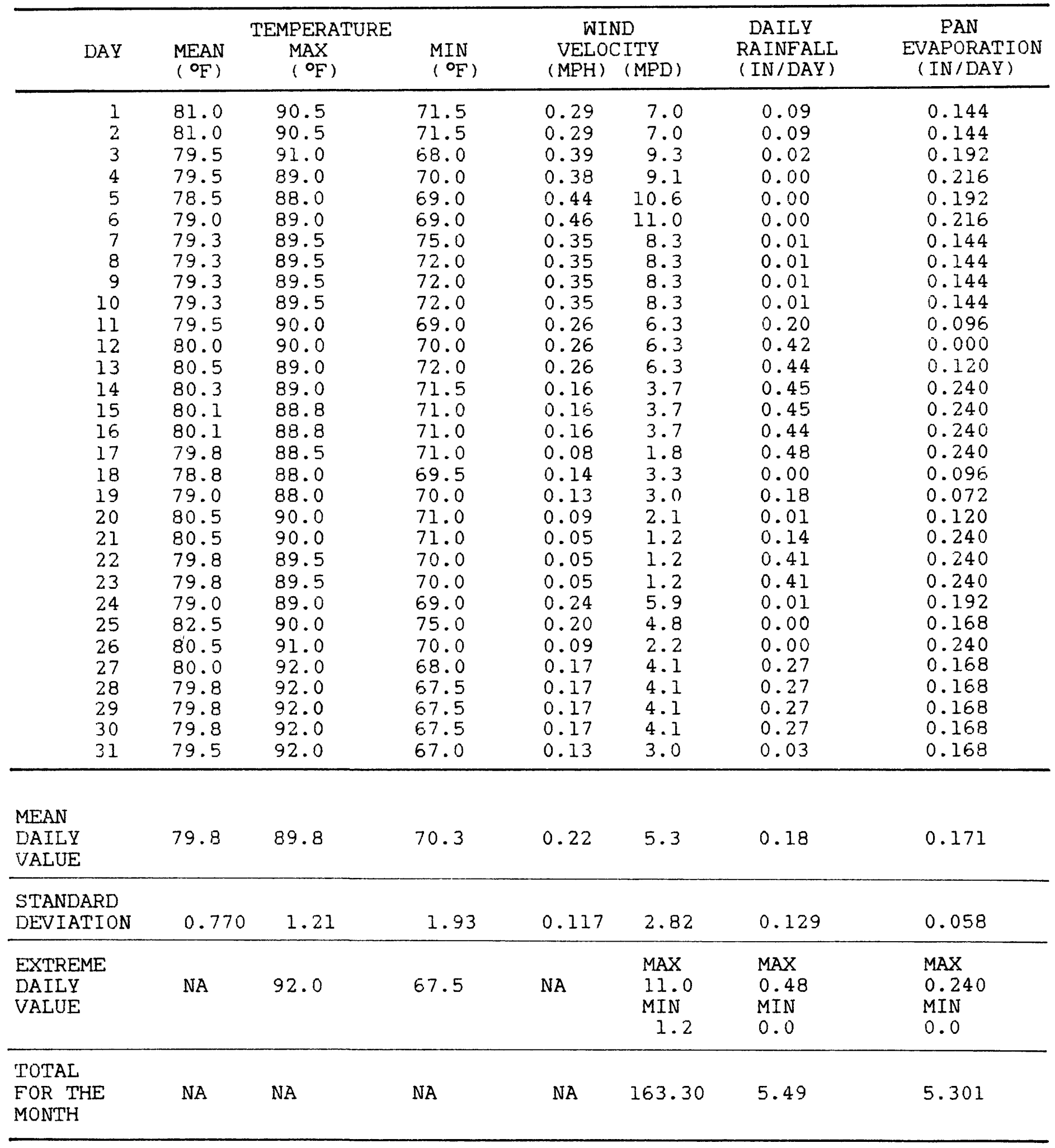


Table 1. (Continued)

VEGA BAJA NOVEMBER 1983

\begin{tabular}{|c|c|c|c|c|c|c|c|}
\hline DAY & $\begin{array}{l}\text { MEAN } \\
\left({ }^{\circ} \mathrm{F}\right)\end{array}$ & $\begin{array}{l}\text { EMPERA } \\
\text { MAX } \\
\left({ }^{\circ} \mathrm{F}\right)\end{array}$ & $\begin{array}{l}\text { MIN } \\
(\text { OF })\end{array}$ & $\begin{array}{l}\text { WIN } \\
\text { VELOC } \\
(\mathrm{MPH})\end{array}$ & $\begin{array}{l}\text { JD } \\
\text { ITY } \\
(M P D)\end{array}$ & $\begin{array}{c}\text { DAILY } \\
\text { RAINEALL } \\
(\text { IN/DAY })\end{array}$ & $\begin{array}{c}\text { PAN } \\
\text { EVAPORATION } \\
(\text { IN/DAY })\end{array}$ \\
\hline $\begin{array}{l}1 \\
2 \\
3 \\
4 \\
5 \\
6 \\
7 \\
8 \\
9 \\
10 \\
11 \\
12 \\
13 \\
14 \\
15 \\
16 \\
17 \\
18 \\
19 \\
20 \\
21 \\
22 \\
23 \\
24 \\
25 \\
26 \\
27 \\
28 \\
29 \\
30\end{array}$ & $\begin{array}{l}78.8 \\
78.0 \\
78.0 \\
79.0 \\
79.4 \\
79.4 \\
79.8 \\
79.0 \\
78.5 \\
76.8 \\
78.2 \\
78.2 \\
78.2 \\
79.5 \\
81.3 \\
81.3 \\
83.0 \\
79.3 \\
78.7 \\
78.7 \\
78.0 \\
77.8 \\
77.5 \\
76.8 \\
76.8 \\
76.8 \\
76.8 \\
76.0 \\
76.0 \\
77.0\end{array}$ & $\begin{array}{l}90.0 \\
88.0 \\
87.0 \\
88.0 \\
90.0 \\
90.0 \\
92.0 \\
88.0 \\
88.0 \\
85.5 \\
89.0 \\
89.0 \\
89.0 \\
93.0 \\
92.5 \\
92.5 \\
92.0 \\
92.0 \\
91.0 \\
91.0 \\
90.0 \\
89.5 \\
89.0 \\
88.5 \\
88.5 \\
88.5 \\
88.5 \\
88.0 \\
87.0 \\
85.0\end{array}$ & $\begin{array}{l}67.5 \\
68.0 \\
69.0 \\
70.0 \\
69.0 \\
69.0 \\
67.5 \\
70.0 \\
69.0 \\
68.0 \\
67.0 \\
67.0 \\
67.0 \\
66.0 \\
70.0 \\
70.0 \\
74.0 \\
66.5 \\
66.0 \\
66.0 \\
66.0 \\
66.0 \\
66.0 \\
65.0 \\
65.0 \\
66.0 \\
65.0 \\
64.0 \\
65.0 \\
69.0\end{array}$ & $\begin{array}{l}0.00 \\
0.07 \\
0.01 \\
0.04 \\
0.04 \\
0.04 \\
0.15 \\
0.20 \\
0.20 \\
0.15 \\
0.15 \\
0.15 \\
0.15 \\
0.23 \\
0.23 \\
0.23 \\
0.22 \\
0.20 \\
0.20 \\
0.20 \\
0.11 \\
0.11 \\
0.02 \\
0.02 \\
0.02 \\
0.02 \\
0.02 \\
0.20 \\
0.12 \\
0.25\end{array}$ & $\begin{array}{l}3.1 \\
1.0 \\
0.2 \\
1.0 \\
1.0 \\
1.0 \\
3.6 \\
4.7 \\
4.7 \\
3.6 \\
3.6 \\
3.6 \\
3.6 \\
5.5 \\
5.5 \\
5.5 \\
5.2 \\
4.9 \\
4.9 \\
4.9 \\
2.7 \\
2.7 \\
0.5 \\
0.5 \\
0.5 \\
0.5 \\
0.5 \\
5.6 \\
2.8 \\
6.0\end{array}$ & $\begin{array}{l}0.03 \\
0.00 \\
0.92 \\
0.42 \\
0.42 \\
0.42 \\
0.02 \\
0.01 \\
0.00 \\
0.01 \\
0.00 \\
0.01 \\
0.00 \\
0.01 \\
0.00 \\
0.00 \\
0.00 \\
0.18 \\
0.18 \\
0.18 \\
0.03 \\
0.03 \\
0.06 \\
0.06 \\
0.06 \\
0.06 \\
0.06 \\
0.00 \\
0.48 \\
0.00\end{array}$ & $\begin{array}{l}0.168 \\
0.168 \\
0.096 \\
0.432 \\
0.432 \\
0.432 \\
0.144 \\
0.168 \\
0.120 \\
0.144 \\
0.144 \\
0.144 \\
0.144 \\
0.168 \\
0.168 \\
0.168 \\
0.048 \\
0.192 \\
0.192 \\
0.192 \\
0.144 \\
0.144 \\
0.144 \\
0.144 \\
0.144 \\
0.144 \\
0.144 \\
0.120 \\
0.072 \\
0.144\end{array}$ \\
\hline $\begin{array}{l}\text { MEAN } \\
\text { DAILY } \\
\text { VALUE }\end{array}$ & 78.4 & 89.3 & 67.4 & 0.12 & 3.1 & 0.12 & 0.174 \\
\hline $\begin{array}{l}\text { STANDARD } \\
\text { DEVIATION }\end{array}$ & 1.56 & 1.99 & 2.11 & 0.080 & 1.930 & 0.210 & 0.091 \\
\hline $\begin{array}{l}\text { EXTREME } \\
\text { DAILY } \\
\text { VALUE }\end{array}$ & NA & 92.5 & 64.0 & NA & $\begin{array}{l}\text { MAX } \\
6.0 \\
\text { MIN } \\
0.2\end{array}$ & $\begin{array}{l}\text { MAX } \\
0.92 \\
\text { MIN } \\
0.0\end{array}$ & $\begin{array}{l}\text { MAX } \\
0.432 \\
\text { MIN } \\
0.048\end{array}$ \\
\hline $\begin{array}{l}\text { TOTAL } \\
\text { FOR THE } \\
\text { MONTH }\end{array}$ & $\mathrm{NA}$ & NA & $\mathrm{NA}$ & $\mathrm{NA}$ & 93.4 & 3.68 & 5.270 \\
\hline
\end{tabular}


Table 1. (Continued)

VEGA BAJA DECEMBER 1983

\begin{tabular}{|c|c|c|c|c|c|c|c|}
\hline DAY & $\begin{array}{l}\text { MEAN } \\
\left({ }^{\circ} \mathrm{F}\right)\end{array}$ & $\begin{array}{l}\text { TEMPERATURE } \\
\text { MAX } \\
(\text { OF })\end{array}$ & $\begin{array}{l}\text { MIN } \\
\left({ }^{\circ} \mathrm{F}\right)\end{array}$ & $\begin{array}{l}\text { WII } \\
\text { VELOC } \\
(\mathrm{MPH})\end{array}$ & $\begin{array}{l}\mathrm{ND} \\
\mathrm{CITY} \\
(\mathrm{MPD})\end{array}$ & $\begin{array}{l}\text { DAILY } \\
\text { RAINFALL } \\
\text { (IN/DAY) }\end{array}$ & $\begin{array}{c}\text { PAN } \\
\text { EVAPORATION } \\
(\text { IN/DAY })\end{array}$ \\
\hline $\begin{array}{l}1 \\
2 \\
3 \\
4 \\
5 \\
6 \\
7 \\
8 \\
9 \\
10 \\
11 \\
12 \\
13 \\
14 \\
15 \\
15 \\
17 \\
18 \\
19 \\
20 \\
21 \\
22 \\
23 \\
24 \\
25 \\
26 \\
27 \\
28 \\
29 \\
30 \\
31\end{array}$ & $\begin{array}{l}77.0 \\
77.5 \\
76.0 \\
76.0 \\
76.5 \\
76.5 \\
76.5 \\
75.5 \\
74.5 \\
74.3 \\
74.3 \\
74.3 \\
74.0 \\
74.5 \\
75.0 \\
77.5 \\
77.0 \\
77.0 \\
76.5 \\
76.0 \\
75.5 \\
77.0 \\
76.8 \\
76.8 \\
76.8 \\
76.8 \\
76.5 \\
76.5 \\
77.5 \\
76.3 \\
76.3\end{array}$ & $\begin{array}{l}87.0 \\
86.0 \\
86.0 \\
86.0 \\
86.0 \\
87.0 \\
88.0 \\
86.0 \\
86.0 \\
86.0 \\
86.0 \\
86.0 \\
86.0 \\
87.0 \\
86.0 \\
86.0 \\
86.0 \\
86.0 \\
86.0 \\
86.0 \\
86.0 \\
86.0 \\
88.0 \\
88.0 \\
88.0 \\
88.0 \\
90.0 \\
86.0 \\
86.0 \\
85.5 \\
85.5\end{array}$ & $\begin{array}{l}67.0 \\
69.0 \\
68.0 \\
68.0 \\
67.0 \\
66.0 \\
65.0 \\
65.0 \\
63.0 \\
62.5 \\
62.5 \\
62.5 \\
62.0 \\
62.0 \\
64.0 \\
69.0 \\
68.0 \\
68.0 \\
67.0 \\
66.0 \\
67.0 \\
68.0 \\
65.5 \\
65.5 \\
65.5 \\
65.5 \\
63.0 \\
67.0 \\
69.0 \\
67.0 \\
67.0\end{array}$ & $\begin{array}{l}0.28 \\
0.35 \\
0.35 \\
0.35 \\
0.24 \\
0.24 \\
0.13 \\
0.13 \\
0.23 \\
0.23 \\
0.23 \\
0.23 \\
0.15 \\
0.30 \\
0.30 \\
0.32 \\
0.32 \\
0.32 \\
0.24 \\
0.17 \\
0.22 \\
0.14 \\
0.14 \\
0.14 \\
0.14 \\
0.14 \\
0.25 \\
0.30 \\
0.18 \\
0.18 \\
0.18\end{array}$ & $\begin{array}{l}6.6 \\
8.5 \\
8.5 \\
8.5 \\
5.8 \\
5.8 \\
3.2 \\
3.2 \\
5.6 \\
5.6 \\
5.6 \\
5.6 \\
3.6 \\
7.2 \\
7.2 \\
7.6 \\
7.6 \\
7.6 \\
5.8 \\
4.2 \\
5.3 \\
3.4 \\
3.4 \\
3.4 \\
3.4 \\
3.4 \\
6.1 \\
7.4 \\
4.2 \\
4.2 \\
4.2\end{array}$ & $\begin{array}{l}0.52 \\
0.11 \\
0.11 \\
0.11 \\
0.13 \\
0.13 \\
0.17 \\
0.17 \\
0.08 \\
0.08 \\
0.08 \\
0.08 \\
0.02 \\
0.16 \\
0.17 \\
0.25 \\
0.25 \\
0.27 \\
0.03 \\
0.00 \\
0.18 \\
0.04 \\
0.04 \\
0.04 \\
0.04 \\
0.04 \\
0.19 \\
0.33 \\
0.29 \\
0.29 \\
0.29\end{array}$ & $\begin{array}{l}0.120 \\
0.144 \\
0.144 \\
0.144 \\
0.024 \\
0.024 \\
0.144 \\
0.144 \\
0.144 \\
0.144 \\
0.144 \\
0.144 \\
0.168 \\
0.168 \\
0.168 \\
0.144 \\
0.144 \\
0.144 \\
0.168 \\
0.144 \\
0.168 \\
0.120 \\
0.120 \\
0.120 \\
0.120 \\
0.120 \\
0.096 \\
0.144 \\
0.168 \\
0.168 \\
0.168\end{array}$ \\
\hline $\begin{array}{l}\text { MEAN } \\
\text { DAILY } \\
\text { VALUE }\end{array}$ & 76.1 & 86.5 & 65.8 & 0.23 & 5.5 & 0.15 & 0.135 \\
\hline $\begin{array}{l}\text { STANDARD } \\
\text { DEVIATION }\end{array}$ & 1.04 & 1.00 & 2.18 & 0.073 & 1.75 & 0.114 & 0.035 \\
\hline $\begin{array}{l}\text { EXTREME } \\
\text { DAILY } \\
\text { VALUE }\end{array}$ & NA & 90.0 & 62.0 & NA & $\begin{array}{l}\text { MAX } \\
8.5 \\
\text { MIN } \\
3.2\end{array}$ & $\begin{array}{l}\text { MAX } \\
0.52 \\
\text { MIN } \\
0.04\end{array}$ & $\begin{array}{l}\text { MAX } \\
0.168 \\
\text { MIN } \\
0.024\end{array}$ \\
\hline $\begin{array}{l}\text { TOTAL } \\
\text { FOR THE } \\
\text { MONTH }\end{array}$ & NA & NA & NA & NA & 168.3 & 4.65 & 4.340 \\
\hline
\end{tabular}


Table 1. (Continued)

VEGA BAJA JANUARY 1984

\begin{tabular}{|c|c|c|c|c|c|c|c|}
\hline DAY & $\begin{array}{l}\text { MEAN } \\
\left({ }^{\circ} \mathrm{F}\right)\end{array}$ & $\begin{array}{c}\text { EMPERA } \\
\text { MAX } \\
\left({ }^{\circ} \mathrm{F}\right)\end{array}$ & $\begin{array}{l}\text { MIN } \\
\left({ }^{\circ} \mathrm{F}\right)\end{array}$ & $\begin{array}{l}\text { WII } \\
\text { VELOC } \\
\text { (MPH) }\end{array}$ & $\begin{array}{l}\text { ND } \\
\text { IIY } \\
(M P D)\end{array}$ & $\begin{array}{c}\text { DAILY } \\
\text { RAINFALL } \\
\text { (IN/DAY) }\end{array}$ & $\begin{array}{c}\text { PAN } \\
\text { EVAPORATION } \\
\text { (IN/DAY) }\end{array}$ \\
\hline $\begin{array}{l}1 \\
2 \\
3 \\
4 \\
5 \\
6 \\
7 \\
8 \\
9 \\
10 \\
11 \\
12 \\
13 \\
14 \\
15 \\
16 \\
17 \\
18 \\
19 \\
20 \\
21 \\
22 \\
23 \\
24 \\
25 \\
26 \\
27 \\
28 \\
29 \\
30 \\
31\end{array}$ & $\begin{array}{l}76.3 \\
76.3 \\
75.0 \\
74.0 \\
75.5 \\
76.3 \\
76.3 \\
76.3 \\
77.0 \\
75.0 \\
74.5 \\
74.8 \\
75.0 \\
75.3 \\
75.3 \\
75.5 \\
76.0 \\
76.5 \\
75.0 \\
73.5 \\
74.0 \\
74.0 \\
74.5 \\
74.0 \\
74.0 \\
74.0 \\
74.0 \\
74.0 \\
74.0 \\
74.0 \\
74.0\end{array}$ & $\begin{array}{l}85.5 \\
85.5 \\
85.0 \\
85.0 \\
84.0 \\
87.0 \\
87.0 \\
87.0 \\
90.0 \\
84.0 \\
84.0 \\
84.5 \\
85.0 \\
85.0 \\
86.0 \\
87.0 \\
87.5 \\
88.0 \\
86.0 \\
84.0 \\
84.5 \\
84.5 \\
85.0 \\
85.0 \\
85.0 \\
85.0 \\
85.0 \\
85.0 \\
85.0 \\
85.0 \\
85.0\end{array}$ & $\begin{array}{l}67.0 \\
67.0 \\
65.0 \\
63.0 \\
67.0 \\
65.5 \\
65.5 \\
65.5 \\
64.0 \\
67.0 \\
65.0 \\
65.0 \\
65.0 \\
64.5 \\
64.5 \\
64.0 \\
64.5 \\
65.0 \\
64.0 \\
63.0 \\
63.5 \\
63.5 \\
64.0 \\
63.0 \\
63.0 \\
63.0 \\
63.0 \\
63.0 \\
63.0 \\
63.0 \\
63.0\end{array}$ & $\begin{array}{l}0.18 \\
0.18 \\
0.04 \\
0.19 \\
0.17 \\
0.17 \\
0.17 \\
0.17 \\
0.19 \\
0.37 \\
0.22 \\
0.22 \\
0.19 \\
0.19 \\
0.19 \\
0.30 \\
0.30 \\
0.33 \\
0.33 \\
0.36 \\
0.36 \\
0.36 \\
0.31 \\
0.31 \\
0.31 \\
0.31 \\
0.31 \\
0.31 \\
0.31 \\
0.31 \\
0.31\end{array}$ & $\begin{array}{l}4.2 \\
4.2 \\
1.0 \\
4.6 \\
4.1 \\
4.1 \\
4.1 \\
4.1 \\
4.6 \\
9.0 \\
5.3 \\
5.3 \\
4.6 \\
4.6 \\
4.6 \\
7.1 \\
7.1 \\
8.0 \\
8.0 \\
8.6 \\
8.6 \\
8.6 \\
7.3 \\
7.3 \\
7.3 \\
7.3 \\
7.3 \\
7.3 \\
7.3 \\
7.3 \\
7.3\end{array}$ & $\begin{array}{l}0.30 \\
0.30 \\
0.02 \\
0.00 \\
0.03 \\
0.03 \\
0.03 \\
0.03 \\
0.28 \\
0.04 \\
0.15 \\
0.16 \\
0.03 \\
0.03 \\
0.03 \\
0.10 \\
0.11 \\
0.19 \\
0.19 \\
0.08 \\
0.08 \\
0.08 \\
0.02 \\
0.00 \\
0.00 \\
0.00 \\
0.00 \\
0.00 \\
0.00 \\
0.00 \\
0.00\end{array}$ & $\begin{array}{l}0.168 \\
0.168 \\
0.144 \\
0.144 \\
0.120 \\
0.120 \\
0.120 \\
0.120 \\
0.120 \\
0.168 \\
0.144 \\
0.144 \\
0.120 \\
0.120 \\
0.120 \\
0.144 \\
0.144 \\
0.120 \\
0.136 \\
0.136 \\
0.136 \\
0.136 \\
0.136 \\
0.136 \\
0.136 \\
0.136 \\
0.136 \\
0.136 \\
0.136 \\
0.136 \\
0.136\end{array}$ \\
\hline $\begin{array}{l}\text { MEAN } \\
\text { DAILY } \\
\text { VALUE }\end{array}$ & 75.0 & 85.5 & 64.4 & 0.26 & 6.1 & 0.07 & 0.131 \\
\hline $\begin{array}{l}\text { STANDARD } \\
\text { DEVIATION }\end{array}$ & 0.986 & 1.33 & 1.32 & 0.080 & 1.89 & 0.091 & 0.015 \\
\hline $\begin{array}{l}\text { EXTREME } \\
\text { DAILY } \\
\text { VALUE }\end{array}$ & $\mathrm{NA}$ & 90.0 & 63.0 & $\mathrm{NA}$ & $\begin{array}{l}\text { MAX } \\
9.0 \\
\text { MIN } \\
1.0\end{array}$ & $\begin{array}{l}\text { MAX } \\
0.30 \\
\text { MIN } \\
0.0\end{array}$ & $\begin{array}{l}\text { MAX } \\
0.168 \\
\text { MIN } \\
0.120\end{array}$ \\
\hline $\begin{array}{l}\text { TOTAL } \\
\text { FOR THE } \\
\text { MONTH }\end{array}$ & $\mathrm{NA}$ & $\mathrm{NA}$ & NA & NA & 188.8 & 2.31 & 4.048 \\
\hline
\end{tabular}


Table 1. (Continued)

VEGA BAJA FEBRUARY 1984

\begin{tabular}{|c|c|c|c|c|c|c|c|}
\hline DAY & $\frac{\text { MEAN }}{\left(\mathrm{O}^{\circ}\right)}$ & $\begin{array}{l}\text { TEMPERATURE } \\
\text { MAX } \\
\text { (OF) }\end{array}$ & $\begin{array}{l}\text { MIN } \\
(\mathrm{OF})\end{array}$ & $\begin{array}{l}\text { WI } \\
\text { VELO } \\
\text { (MPH) }\end{array}$ & $\begin{array}{l}\text { ND } \\
\text { ITYY } \\
\text { (MPD) }\end{array}$ & $\begin{array}{l}\text { DAILY } \\
\text { RAINFALL } \\
\text { (IN/DAY) }\end{array}$ & $\begin{array}{l}\text { PAN } \\
\text { EVAPORATICN } \\
\text { (IN / DAY) }\end{array}$ \\
\hline $\begin{array}{l}1 \\
2 \\
3 \\
4 \\
5 \\
6 \\
7 \\
8 \\
9 \\
10 \\
11 \\
12 \\
13 \\
14 \\
15 \\
16 \\
17 \\
18 \\
19 \\
20 \\
21 \\
22 \\
23 \\
24 \\
25 \\
26 \\
27 \\
28 \\
29\end{array}$ & $\begin{array}{l}74.0 \\
74.0 \\
73.5 \\
75.3 \\
75.3 \\
77.0 \\
77.0 \\
74.5 \\
77.5 \\
77.0 \\
75.3 \\
75.3 \\
73.5 \\
74.0 \\
74.0 \\
76.0 \\
77.5 \\
75.5 \\
75.5 \\
75.5 \\
75.5 \\
75.5 \\
75.5 \\
75.5 \\
75.5 \\
75.5 \\
75.5 \\
75.5 \\
75.5\end{array}$ & $\begin{array}{l}85.0 \\
85.0 \\
85.0 \\
88.5 \\
88.5 \\
92.0 \\
89.0 \\
86.0 \\
86.0 \\
85.0 \\
83.5 \\
83.5 \\
82.0 \\
83.0 \\
83.0 \\
84.0 \\
85.0 \\
86.0 \\
86.0 \\
86.0 \\
86.0 \\
86.0 \\
86.0 \\
86.0 \\
86.0 \\
86.0 \\
86.0 \\
86.0 \\
86.0\end{array}$ & $\begin{array}{l}63.0 \\
63.0 \\
62.0 \\
62.0 \\
62.0 \\
52.0 \\
65.0 \\
63.0 \\
68.0 \\
69.0 \\
67.0 \\
67.0 \\
65.0 \\
65.0 \\
55.0 \\
68.0 \\
70.0 \\
70.5 \\
70.5 \\
70.5 \\
70.5 \\
70.5 \\
70.5 \\
70.5 \\
70.5 \\
70.5 \\
70.5 \\
70.5 \\
70.5\end{array}$ & $\begin{array}{l}0.31 \\
0.31 \\
0.33 \\
0.33 \\
0.33 \\
0.54 \\
0.26 \\
0.40 \\
0.34 \\
1.03 \\
1.03 \\
1.03 \\
0.77 \\
0.91 \\
1.34 \\
1.07 \\
0.79 \\
0.79 \\
0.79 \\
0.79 \\
0.79 \\
0.79 \\
0.79 \\
0.79 \\
0.79 \\
0.79 \\
0.79 \\
0.79 \\
0.79\end{array}$ & $\begin{array}{r}7.3 \\
7.3 \\
8.0 \\
8.0 \\
8.0 \\
12.8 \\
6.2 \\
9.6 \\
8.1 \\
24.7 \\
24.7 \\
24.7 \\
18.5 \\
21.7 \\
32.2 \\
25.7 \\
19.0 \\
19.0 \\
19.0 \\
19.0 \\
19.0 \\
19.0 \\
19.0 \\
19.0 \\
19.0 \\
19.0 \\
19.0 \\
19.0 \\
19.0\end{array}$ & $\begin{array}{l}0.00 \\
0.00 \\
0.00 \\
0.00 \\
0.00 \\
0.11 \\
0.00 \\
0.48 \\
1.43 \\
0.83 \\
0.83 \\
0.85 \\
0.52 \\
0.01 \\
0.82 \\
0.00 \\
0.00 \\
0.00 \\
0.00 \\
0.00 \\
0.00 \\
0.00 \\
0.00 \\
0.00 \\
0.00 \\
0.00 \\
0.00 \\
0.00 \\
0.00\end{array}$ & $\begin{array}{l}0.136 \\
0.136 \\
0.024 \\
0.024 \\
0.024 \\
0.552 \\
0.264 \\
0.336 \\
0.336 \\
0.384 \\
0.384 \\
0.384 \\
0.096 \\
0.216 \\
0.168 \\
0.048 \\
0.048 \\
0.048 \\
0.048 \\
0.048 \\
0.048 \\
0.048 \\
0.048 \\
0.048 \\
0.048 \\
0.048 \\
0.048 \\
0.048 \\
0.048\end{array}$ \\
\hline $\begin{array}{l}\text { MEAN } \\
\text { DAILY } \\
\text { VALUE }\end{array}$ & 75.4 & 85.7 & 67.3 & 0.71 & 17.0 & 0.20 & 0.164 \\
\hline $\begin{array}{l}\text { STANDARD } \\
\text { DEVIATION }\end{array}$ & 1.06 & 1.95 & 3.34 & 0.274 & 6.59 & 0.377 & 0.146 \\
\hline $\begin{array}{l}\text { EXTREME } \\
\text { DAILY } \\
\text { VALUE }\end{array}$ & NA & 92.0 & 62.0 & NA & $\begin{array}{l}\text { MAX } \\
25.7 \\
\text { MIN } \\
6.2\end{array}$ & $\begin{array}{l}\text { MAX } \\
1.43 \\
\text { MIN } \\
0.0\end{array}$ & $\begin{array}{l}\text { MAX } \\
0.552 \\
\text { MIN } \\
0.024\end{array}$ \\
\hline $\begin{array}{l}\text { TOTAL } \\
\text { FOR THE } \\
\text { MONTH }\end{array}$ & NA & $\mathrm{NA}$ & $\mathrm{NA}$ & NA & 494.50 & 5.88 & 4.784 \\
\hline
\end{tabular}


Table 1. (Continued)

VEGA BAJA MARCH 1984

\begin{tabular}{|c|c|c|c|c|c|c|c|}
\hline DAY & $\begin{array}{l}\text { MEAN } \\
(\text { OF })\end{array}$ & $\begin{array}{c}\text { TEMPERA? } \\
\text { MAX } \\
(\mathrm{OF})\end{array}$ & $\begin{array}{l}\text { MIN } \\
(\text { OF })\end{array}$ & $\begin{array}{r}\text { WI } \\
\text { VELO } \\
\text { (MPH) }\end{array}$ & $\begin{array}{l}\text { ND } \\
\text { CITY } \\
\text { (MPD) }\end{array}$ & $\begin{array}{c}\text { DAILY } \\
\text { RAINFALL } \\
\text { (IN/DAY) }\end{array}$ & $\begin{array}{c}\text { PAN } \\
\text { EVAPORATION } \\
(\text { IN / DAY })\end{array}$ \\
\hline $\begin{array}{l}1 \\
2 \\
3 \\
4 \\
5 \\
6 \\
7 \\
8 \\
9 \\
10 \\
11 \\
12 \\
13 \\
14 \\
15 \\
16 \\
17 \\
18 \\
19 \\
20 \\
21 \\
22 \\
23 \\
24 \\
25 \\
26 \\
27 \\
28 \\
29 \\
30 \\
31\end{array}$ & $\begin{array}{l}75.5 \\
75.5 \\
75.5 \\
75.5 \\
75.5 \\
75.5 \\
75.5 \\
75.5 \\
75.3 \\
74.6 \\
74.6 \\
74.6 \\
73.8 \\
76.5 \\
74.8 \\
75.5 \\
73.9 \\
73.9 \\
72.3 \\
73.5 \\
73.0 \\
76.5 \\
78.0 \\
77.0 \\
77.0 \\
76.0 \\
77.3 \\
75.0 \\
78.3 \\
79.5 \\
79.1\end{array}$ & $\begin{array}{l}86.5 \\
86.5 \\
86.5 \\
86.5 \\
86.5 \\
86.5 \\
86.5 \\
86.5 \\
88.0 \\
86.8 \\
86.8 \\
86.8 \\
85.5 \\
85.0 \\
84.5 \\
85.0 \\
84.5 \\
84.5 \\
84.0 \\
84.0 \\
84.0 \\
88.0 \\
86.0 \\
88.0 \\
88.0 \\
90.0 \\
90.0 \\
87.0 \\
91.0 \\
94.5 \\
91.3\end{array}$ & $\begin{array}{l}66.3 \\
66.3 \\
66.3 \\
66.3 \\
66.3 \\
66.3 \\
66.3 \\
66.3 \\
62.5 \\
62.3 \\
62.3 \\
62.3 \\
62.0 \\
68.0 \\
65.0 \\
66.0 \\
63.3 \\
63.3 \\
60.5 \\
63.0 \\
62.0 \\
65.0 \\
70.0 \\
66.0 \\
66.0 \\
62.0 \\
64.5 \\
63.0 \\
65.5 \\
64.5 \\
66.8\end{array}$ & $\begin{array}{l}0.31 \\
0.31 \\
0.31 \\
0.31 \\
0.31 \\
0.31 \\
0.31 \\
0.31 \\
4.44 \\
4.44 \\
4.44 \\
4.44 \\
2.00 \\
1.59 \\
1.59 \\
1.08 \\
1.08 \\
1.08 \\
1.38 \\
1.41 \\
1.43 \\
2.07 \\
1.42 \\
1.42 \\
1.42 \\
1.05 \\
2.12 \\
1.55 \\
1.70 \\
1.10 \\
1.10\end{array}$ & $\begin{array}{r}7.3 \\
7.3 \\
7.3 \\
7.3 \\
7.3 \\
7.3 \\
7.3 \\
7.3 \\
106.5 \\
106.5 \\
106.5 \\
106.5 \\
48.0 \\
38.2 \\
38.2 \\
25.8 \\
25.8 \\
25.8 \\
33.0 \\
33.9 \\
34.2 \\
49.7 \\
34.1 \\
34.1 \\
34.1 \\
25.1 \\
50.8 \\
37.1 \\
40.8 \\
26.3 \\
26.3\end{array}$ & $\begin{array}{l}0.00 \\
0.00 \\
0.00 \\
0.00 \\
0.00 \\
0.00 \\
0.00 \\
0.00 \\
0.05 \\
0.05 \\
0.06 \\
0.06 \\
0.11 \\
0.00 \\
0.02 \\
0.02 \\
0.03 \\
0.20 \\
0.00 \\
0.00 \\
0.00 \\
0.00 \\
0.00 \\
0.00 \\
0.00 \\
0.00 \\
0.00 \\
0.00 \\
0.00 \\
0.00 \\
0.00\end{array}$ & $\begin{array}{l}0.048 \\
0.048 \\
0.048 \\
0.048 \\
0.048 \\
0.048 \\
0.048 \\
0.048 \\
0.096 \\
0.096 \\
0.096 \\
0.096 \\
0.264 \\
0.288 \\
0.288 \\
0.336 \\
0.336 \\
0.336 \\
0.048 \\
0.288 \\
0.240 \\
0.264 \\
0.288 \\
0.288 \\
0.288 \\
0.216 \\
0.288 \\
0.264 \\
0.336 \\
0.264 \\
0.264\end{array}$ \\
\hline $\begin{array}{l}\text { MEAN } \\
\text { DAILY } \\
\text { VALUE }\end{array}$ & 75.6 & 86.9 & 64.7 & 1.54 & 37.0 & 0.02 & 0.192 \\
\hline $\begin{array}{l}\text { STANDARD } \\
\text { DEVIATION }\end{array}$ & 1.65 & 2.35 & 2.13 & 1.24 & 29.9 & 0.042 & 0.114 \\
\hline $\begin{array}{l}\text { EXTREME } \\
\text { DAILY } \\
\text { VALUE }\end{array}$ & NA & 94.5 & 60.5 & NA & $\begin{array}{l}\text { MAX } \\
106.5 \\
\text { MIN } \\
7.3\end{array}$ & $\begin{array}{l}\text { MAX } \\
0.20 \\
\text { MIN } \\
0.0\end{array}$ & $\begin{array}{l}\text { MAX } \\
0.336 \\
\text { MIN } \\
0.048\end{array}$ \\
\hline $\begin{array}{l}\text { TOTAL } \\
\text { FOR THE } \\
\text { MONTH }\end{array}$ & NA & NA & NA & $\mathrm{NA}$ & 1145.7 & 0.60 & 5.956 \\
\hline
\end{tabular}


Table 1. (Contlnued)

VEGA BAJA APRIL 1984

\begin{tabular}{|c|c|c|c|c|c|c|c|}
\hline DAY & $\begin{array}{l}\text { MEAN } \\
\left({ }^{\circ} \mathrm{F}\right)\end{array}$ & $\begin{array}{c}\text { TEMPERA } \\
\text { MAX } \\
\left({ }^{\circ} \mathrm{F}\right)\end{array}$ & $\begin{array}{l}\text { MIN } \\
(O F)\end{array}$ & $\begin{array}{r}\text { WI } \\
\text { VELO } \\
(M P H)\end{array}$ & $\begin{array}{l}\text { ND } \\
\text { CITY } \\
\text { (MPD) }\end{array}$ & $\begin{array}{l}\text { DAILY } \\
\text { RAINFALL } \\
\text { (IN/DAY) }\end{array}$ & $\begin{array}{c}\text { PAN } \\
\text { EVAPORATION } \\
(\text { IN / DAY })\end{array}$ \\
\hline $\begin{array}{l}1 \\
2 \\
3 \\
4 \\
5 \\
6 \\
7 \\
8 \\
9 \\
10 \\
11 \\
12 \\
13 \\
14 \\
15 \\
16 \\
17 \\
18 \\
19 \\
20 \\
21 \\
22 \\
23 \\
24 \\
25 \\
26 \\
27 \\
28 \\
29 \\
30\end{array}$ & $\begin{array}{l}79.1 \\
78.5 \\
78.5 \\
75.5 \\
77.5 \\
78.8 \\
78.7 \\
78.7 \\
78.5 \\
77.0 \\
80.5 \\
78.0 \\
78.0 \\
77.5 \\
77.5 \\
77.0 \\
78.0 \\
77.0 \\
78.0 \\
77.3 \\
77.3 \\
77.3 \\
76.5 \\
77.0 \\
78.0 \\
79.0 \\
78.5 \\
76.8 \\
76.8 \\
75.0\end{array}$ & $\begin{array}{l}91.3 \\
90.0 \\
88.0 \\
86.0 \\
92.0 \\
93.5 \\
94.3 \\
94.3 \\
95.0 \\
93.0 \\
94.0 \\
90.0 \\
90.0 \\
90.0 \\
90.0 \\
90.0 \\
91.0 \\
90.0 \\
90.0 \\
89.0 \\
89.0 \\
89.0 \\
88.0 \\
90.0 \\
91.0 \\
90.0 \\
89.0 \\
88.5 \\
88.5 \\
88.0\end{array}$ & $\begin{array}{l}66.8 \\
67.0 \\
69.0 \\
65.0 \\
63.0 \\
64.0 \\
63.0 \\
63.0 \\
62.0 \\
61.0 \\
67.0 \\
66.0 \\
66.0 \\
65.0 \\
65.0 \\
64.0 \\
65.0 \\
64.0 \\
66.0 \\
65.5 \\
65.5 \\
65.5 \\
65.0 \\
64.0 \\
55.0 \\
68.0 \\
68.0 \\
66.0 \\
66.0 \\
64.0\end{array}$ & $\begin{array}{l}0.00 \\
1.02 \\
1.13 \\
2.09 \\
1.13 \\
1.47 \\
1.47 \\
1.47 \\
1.88 \\
0.98 \\
1.38 \\
0.66 \\
2.01 \\
2.01 \\
2.01 \\
1.23 \\
1.75 \\
2.03 \\
2.05 \\
2.05 \\
2.05 \\
2.05 \\
1.83 \\
1.61 \\
1.23 \\
0.96 \\
1.52 \\
1.52 \\
1.52 \\
1.09\end{array}$ & $\begin{array}{l}37.4 \\
24.4 \\
27.1 \\
50.0 \\
27.1 \\
35.2 \\
35.2 \\
35.2 \\
45.2 \\
23.4 \\
33.2 \\
15.9 \\
48.2 \\
48.2 \\
48.2 \\
29.5 \\
42.1 \\
48.7 \\
49.2 \\
49.2 \\
49.2 \\
49.2 \\
44.0 \\
38.7 \\
29.4 \\
22.9 \\
36.5 \\
36.5 \\
36.5 \\
26.2\end{array}$ & $\begin{array}{l}0.00 \\
0.22 \\
0.05 \\
0.00 \\
0.00 \\
0.00 \\
0.00 \\
0.00 \\
0.00 \\
0.00 \\
0.00 \\
0.00 \\
0.00 \\
0.00 \\
0.00 \\
0.00 \\
0.00 \\
0.00 \\
0.01 \\
0.01 \\
0.01 \\
0.01 \\
0.00 \\
0.00 \\
0.00 \\
0.06 \\
0.00 \\
0.00 \\
0.00 \\
0.06\end{array}$ & $\begin{array}{l}0.264 \\
0.264 \\
0.288 \\
0.192 \\
0.144 \\
0.264 \\
0.264 \\
0.254 \\
0.312 \\
0.264 \\
0.312 \\
0.168 \\
0.312 \\
0.312 \\
0.312 \\
0.216 \\
0.264 \\
0.312 \\
0.288 \\
0.288 \\
0.288 \\
0.288 \\
0.288 \\
0.336 \\
0.216 \\
0.288 \\
0.264 \\
0.254 \\
0.264 \\
0.288\end{array}$ \\
\hline $\begin{array}{l}\text { MEAN } \\
\text { DAILY } \\
\text { VALUE }\end{array}$ & 77.7 & 90.4 & 65.1 & 1.51 & 37.4 & 0.01 & 0.269 \\
\hline $\begin{array}{l}\text { STANDARD } \\
\text { DEVIATION }\end{array}$ & 1.09 & 2.15 & 1.76 & 0.493 & 9.76 & 0.042 & 0.043 \\
\hline $\begin{array}{l}\text { EXTREME } \\
\text { DAILY } \\
\text { VALUE }\end{array}$ & $\mathrm{NA}$ & 95.0 & 61.0 & NA & $\begin{array}{l}\text { MAX } \\
50.0 \\
\text { MIN } \\
15.9\end{array}$ & $\begin{array}{l}\text { MAX } \\
0.22 \\
\text { MIN } \\
0.0\end{array}$ & $\begin{array}{l}\text { MAX } \\
0.336 \\
\text { MIN } \\
0.144\end{array}$ \\
\hline $\begin{array}{l}\text { TOTAL } \\
\text { FOR THE } \\
\text { MONTH }\end{array}$ & NA & NA & NA & NA & 1121.7 & 0.43 & 8.088 \\
\hline
\end{tabular}


Tabie 1. (Continued)

VEGA BAJA MAY 1984

\begin{tabular}{|c|c|c|c|c|c|c|c|}
\hline DAY & $\begin{array}{l}\text { MEAN } \\
\left({ }^{\circ} \mathrm{F}\right)\end{array}$ & $\begin{array}{l}\text { TEMPERATURE } \\
\text { MAX } \\
\left(\mathrm{O}^{\circ}\right)\end{array}$ & $\begin{array}{l}\text { MIN } \\
\left({ }^{\circ} \mathrm{F}\right)\end{array}$ & $\begin{array}{l}\text { WII } \\
\text { VELOC } \\
\text { (MPH) }\end{array}$ & $\begin{array}{l}\text { ND } \\
\text { CITY } \\
\text { (MPD) }\end{array}$ & $\begin{array}{l}\text { DAILY } \\
\text { RAINFALL } \\
\text { (IN/DAY) }\end{array}$ & $\begin{array}{c}\text { PAN } \\
\text { EVAPORATION } \\
\text { (IN/DAY) }\end{array}$ \\
\hline $\begin{array}{l}1 \\
2 \\
3 \\
4 \\
5 \\
6 \\
7 \\
8 \\
9 \\
10 \\
11 \\
12 \\
13 \\
14 \\
15 \\
16 \\
17 \\
18 \\
19 \\
20 \\
21 \\
22 \\
23 \\
24 \\
25 \\
26 \\
27 \\
28 \\
29 \\
30 \\
31\end{array}$ & $\begin{array}{l}75.5 \\
77.5 \\
81.0 \\
81.0 \\
78.8 \\
78.8 \\
77.5 \\
77.0 \\
79.0 \\
78.0 \\
80.0 \\
78.5 \\
78.5 \\
77.0 \\
78.0 \\
77.5 \\
78.0 \\
80.0 \\
79.3 \\
79.3 \\
73.5 \\
79.5 \\
76.5 \\
78.5 \\
77.5 \\
78.2 \\
78.2 \\
78.2 \\
78.8 \\
78.5 \\
79.5\end{array}$ & $\begin{array}{l}85.0 \\
85.0 \\
88.0 \\
88.0 \\
88.5 \\
88.5 \\
89.0 \\
89.0 \\
88.5 \\
88.0 \\
91.0 \\
89.5 \\
89.5 \\
88.0 \\
88.0 \\
87.0 \\
88.0 \\
91.0 \\
89.5 \\
89.5 \\
88.0 \\
89.0 \\
83.0 \\
88.0 \\
86.0 \\
86.8 \\
86.8 \\
86.8 \\
87.5 \\
86.0 \\
90.0\end{array}$ & $\begin{array}{l}66.0 \\
70.0 \\
74.0 \\
72.0 \\
69.0 \\
69.0 \\
66.0 \\
65.0 \\
69.5 \\
68.0 \\
69.0 \\
67.5 \\
67.5 \\
66.0 \\
68.0 \\
68.0 \\
68.0 \\
69.0 \\
69.0 \\
69.0 \\
69.0 \\
71.0 \\
70.0 \\
69.0 \\
69.0 \\
69.5 \\
69.5 \\
69.5 \\
70.0 \\
71.0 \\
73.0\end{array}$ & $\begin{array}{l}1.29 \\
2.79 \\
2.12 \\
2.13 \\
2.13 \\
2.13 \\
1.74 \\
1.89 \\
1.54 \\
1.90 \\
1.60 \\
1.60 \\
1.60 \\
1.83 \\
1.54 \\
1.52 \\
1.19 \\
1.22 \\
1.22 \\
1.22 \\
1.77 \\
1.73 \\
1.68 \\
1.44 \\
1.14 \\
1.14 \\
1.14 \\
1.14 \\
1.17 \\
1.03 \\
1.01\end{array}$ & $\begin{array}{l}30.9 \\
66.9 \\
50.8 \\
51.2 \\
51.2 \\
51.2 \\
41.9 \\
45.4 \\
37.0 \\
45.7 \\
38.4 \\
38.4 \\
38.4 \\
43.8 \\
36.9 \\
36.4 \\
28.5 \\
29.4 \\
29.4 \\
29.4 \\
42.5 \\
41.6 \\
40.3 \\
34.5 \\
27.4 \\
27.4 \\
27.4 \\
27.4 \\
28.0 \\
24.7 \\
24.2\end{array}$ & $\begin{array}{l}0.00 \\
0.08 \\
0.04 \\
0.03 \\
0.03 \\
0.04 \\
0.00 \\
0.23 \\
0.00 \\
0.13 \\
0.03 \\
0.04 \\
0.05 \\
0.07 \\
0.01 \\
0.00 \\
1.06 \\
0.20 \\
0.20 \\
0.20 \\
0.04 \\
0.10 \\
0.44 \\
0.01 \\
0.22 \\
0.22 \\
0.22 \\
0.24 \\
0.09 \\
0.00 \\
0.00\end{array}$ & $\begin{array}{l}0.192 \\
0.408 \\
0.192 \\
0.360 \\
0.360 \\
0.360 \\
0.312 \\
0.288 \\
0.360 \\
0.240 \\
0.288 \\
0.288 \\
0.288 \\
0.288 \\
0.288 \\
0.312 \\
0.384 \\
0.216 \\
0.216 \\
0.216 \\
0.288 \\
0.240 \\
0.240 \\
0.144 \\
0.216 \\
0.216 \\
0.216 \\
0.216 \\
0.168 \\
0.192 \\
0.288\end{array}$ \\
\hline $\begin{array}{l}\text { MEAN } \\
\text { DAILY } \\
\text { VALUE }\end{array}$ & 78.4 & 87.9 & 69.0 & 1.57 & 37.6 & 0.13 & 0.267 \\
\hline $\begin{array}{l}\text { STANDARD } \\
\text { DEVIATION }\end{array}$ & 1.18 & 1.72 & 1.91 & 0.410 & 9.86 & 0.200 & 0.067 \\
\hline $\begin{array}{l}\text { EXTREME } \\
\text { DAILY } \\
\text { VALUE }\end{array}$ & NA & 91.0 & 65.0 & $\mathrm{NA}$ & $\begin{array}{l}\text { MAX } \\
66.9 \\
\text { MIN } \\
24.2\end{array}$ & $\begin{array}{l}\text { MAX } \\
1.06 \\
\text { MIN } \\
0.0\end{array}$ & $\begin{array}{l}\text { MAX } \\
4.080 \\
\text { MIN } \\
0.144\end{array}$ \\
\hline $\begin{array}{l}\text { TOTAL } \\
\text { FOR THE } \\
\text { MONTH }\end{array}$ & NA & NA & NA & $\mathrm{NA}$ & 1166.6 & 4.07 & 8.277 \\
\hline
\end{tabular}


Table 1. (Continued)

VEGA BAJA JUNE 1984

\begin{tabular}{|c|c|c|c|c|c|c|c|}
\hline DAY & $\frac{\text { MEAN }}{\left({ }^{\circ} \mathrm{F}\right)}$ & $\begin{array}{c}\text { [EMPERA' } \\
\text { MAX } \\
\left({ }^{\circ} \mathrm{F}\right)\end{array}$ & $\begin{array}{l}\text { MIN } \\
\left({ }^{\circ} \mathrm{F}\right)\end{array}$ & $\begin{array}{c}\text { WII } \\
\text { VELOC } \\
(\mathrm{MPH})\end{array}$ & $\begin{array}{l}\text { JD } \\
\text { (MPY } \\
\text { (MPD) }\end{array}$ & $\begin{array}{c}\text { DAILY } \\
\text { RAINFALL } \\
\text { (IN/DAY ) }\end{array}$ & $\begin{array}{c}\text { PAN } \\
\text { EVAPORATION } \\
(\text { IN/DAY })\end{array}$ \\
\hline $\begin{array}{l}1 \\
2 \\
3 \\
4 \\
5 \\
6 \\
7 \\
8 \\
9 \\
10 \\
11 \\
12 \\
13 \\
14 \\
15 \\
16 \\
17 \\
18 \\
19 \\
20 \\
21 \\
22 \\
23 \\
24 \\
25 \\
26 \\
27 \\
28 \\
29 \\
30\end{array}$ & $\begin{array}{l}81.4 \\
81.4 \\
81.4 \\
81.4 \\
83.5 \\
81.0 \\
83.0 \\
73.8 \\
79.1 \\
79 \\
79.1 \\
79.3 \\
79.0 \\
79.0 \\
77.0 \\
79.5 \\
79.5 \\
79.5 \\
791.0 \\
77.0 \\
78.5 \\
78.0 \\
79.0 \\
79.0 \\
79.3 \\
79.3\end{array}$ & $\begin{array}{l}92.5 \\
92.5 \\
92.5 \\
92.5 \\
95.0 \\
90.0 \\
84.0 \\
86.5 \\
87.5 \\
87.5 \\
88.5 \\
87.0 \\
86.5 \\
86.0 \\
88.0 \\
88.0 \\
88.0 \\
90.0 \\
87.5 \\
87.0 \\
88.0 \\
88.0 \\
88.0 \\
88.0 \\
89.0 \\
89.0 \\
89.0 \\
89.0 \\
88.0 \\
89.0\end{array}$ & $\begin{array}{l}72.5 \\
72.5 \\
72.5 \\
72.5 \\
72.0 \\
72.0 \\
72.0 \\
71.0 \\
70.5 \\
70.5 \\
70.0 \\
71.0 \\
73.0 \\
70.0 \\
71.0 \\
71.0 \\
71.0 \\
72.0 \\
69.5 \\
69.0 \\
70.0 \\
70.0 \\
70.0 \\
70.0 \\
70.0 \\
70.0 \\
70.0 \\
72.0 \\
69.0 \\
70.0\end{array}$ & $\begin{array}{l}1.01 \\
1.01 \\
1.01 \\
1.01 \\
0.52 \\
0.10 \\
0.22 \\
0.51 \\
0.51 \\
0.51 \\
0.77 \\
1.10 \\
0.26 \\
0.97 \\
0.97 \\
0.97 \\
0.97 \\
0.44 \\
0.50 \\
0.95 \\
0.74 \\
0.99 \\
0.99 \\
0.99 \\
1.27 \\
1.27 \\
1.96 \\
1.08 \\
2.00 \\
2.00\end{array}$ & $\begin{array}{r}24.2 \\
24.2 \\
24.2 \\
24.2 \\
12.6 \\
2.4 \\
5.4 \\
12.2 \\
12.2 \\
12.2 \\
18.4 \\
26.3 \\
6.1 \\
23.4 \\
23.4 \\
23.4 \\
23.4 \\
10.5 \\
12.1 \\
22.8 \\
17.8 \\
23.7 \\
23.7 \\
23.7 \\
30.4 \\
30.4 \\
47.2 \\
26.0 \\
48.1 \\
48.1\end{array}$ & $\begin{array}{l}0.00 \\
0.00 \\
0.00 \\
0.01 \\
1.48 \\
0.05 \\
0.78 \\
0.01 \\
0.01 \\
0.01 \\
0.11 \\
0.34 \\
0.25 \\
0.00 \\
0.00 \\
0.00 \\
0.00 \\
0.01 \\
0.01 \\
0.00 \\
0.00 \\
0.00 \\
0.00 \\
0.00 \\
0.00 \\
0.00 \\
0.05 \\
0.02 \\
0.07 \\
0.07\end{array}$ & $\begin{array}{l}0.288 \\
0.288 \\
0.288 \\
0.288 \\
0.216 \\
0.120 \\
0.168 \\
0.168 \\
0.168 \\
0.168 \\
0.192 \\
0.312 \\
0.192 \\
0.168 \\
0.168 \\
0.168 \\
0.168 \\
0.144 \\
0.168 \\
0.192 \\
0.168 \\
0.242 \\
0.242 \\
0.242 \\
0.264 \\
0.264 \\
0.168 \\
0.168 \\
0.240 \\
0.240\end{array}$ \\
\hline $\begin{array}{l}\text { MEAN } \\
\text { DAILY } \\
\text { VALUE }\end{array}$ & 79.8 & 84.4 & 70.9 & 0.92 & 22.1 & 0.11 & 0.207 \\
\hline $\begin{array}{l}\text { STANDARD } \\
\text { DEVIATION }\end{array}$ & 1.33 & 2.28 & 1.13 & 0.465 & 11.2 & 0.296 & 0.052 \\
\hline $\begin{array}{l}\text { EXTREME } \\
\text { DAILY } \\
\text { VALUE }\end{array}$ & NA & 92.5 & 59.0 & NA & $\begin{array}{l}\text { MAX } \\
48.1 \\
\text { MIN } \\
2.4\end{array}$ & $\begin{array}{l}\text { MAX } \\
1.48 \\
\text { MIN } \\
0.0\end{array}$ & $\begin{array}{l}\text { MAX } \\
0.312 \\
\text { MIN } \\
0.120\end{array}$ \\
\hline $\begin{array}{l}\text { TOTAL } \\
\text { FOR THE } \\
\text { MONTH }\end{array}$ & $\mathrm{NA}$ & NA & NA & NA & 662.7 & 3.30 & 6.300 \\
\hline
\end{tabular}


Table 1. (Continued)

VEGA BAJA JULY 1984

\begin{tabular}{|c|c|c|c|c|c|c|c|}
\hline DAY & $\frac{\text { MEAN }}{\left({ }^{\circ} \mathrm{F}\right)}$ & $\begin{array}{l}\text { TEMPERATURE } \\
\text { MAX } \\
\left({ }^{\circ} \mathrm{F}\right)\end{array}$ & $\begin{array}{l}\text { MIN } \\
\left({ }^{\circ} \mathrm{F}\right)\end{array}$ & $\begin{array}{r}\text { WI } \\
\text { VELO } \\
(\mathrm{MPH})\end{array}$ & $\begin{array}{l}\text { ND } \\
\text { ITY } \\
\text { (MPD) }\end{array}$ & $\begin{array}{l}\text { DAILY } \\
\text { RAINFALI } \\
(\text { IN/DAY })\end{array}$ & $\begin{array}{c}\text { PAN } \\
\text { EVAPORATION } \\
\text { (IN/DAY })\end{array}$ \\
\hline $\begin{array}{l}1 \\
2 \\
3 \\
4 \\
5 \\
6 \\
7 \\
8 \\
9 \\
10 \\
11 \\
12 \\
13 \\
14 \\
15 \\
16 \\
17 \\
18 \\
19 \\
20 \\
21 \\
22 \\
23 \\
24 \\
25 \\
26 \\
27 \\
28 \\
29 \\
30 \\
31\end{array}$ & $\begin{array}{l}79.5 \\
80.5 \\
77.5 \\
77.5 \\
74.5 \\
79.5 \\
79.5 \\
79.5 \\
79.5 \\
78.5 \\
78.1 \\
77.6 \\
80.0 \\
80.5 \\
80.5 \\
81.0 \\
80.0 \\
79.0 \\
78.3 \\
77.5 \\
78.0 \\
78.0 \\
78.5 \\
80.3 \\
81.5 \\
82.3 \\
81.4 \\
81.0 \\
81.0 \\
80.5 \\
81.0\end{array}$ & $\begin{array}{l}88.7 \\
90.0 \\
89.0 \\
89.0 \\
78.0 \\
87.0 \\
87.5 \\
87.5 \\
88.0 \\
88.0 \\
86.5 \\
85.0 \\
88.0 \\
90.0 \\
90.0 \\
92.0 \\
90.0 \\
88.0 \\
87.5 \\
87.0 \\
87.0 \\
87.0 \\
87.0 \\
86.5 \\
87.0 \\
87.5 \\
90.3 \\
90.7 \\
90.7 \\
91.0 \\
90.0\end{array}$ & $\begin{array}{l}70.0 \\
71.0 \\
71.0 \\
71.0 \\
71.0 \\
72.0 \\
71.5 \\
71.5 \\
71.0 \\
59.0 \\
69.6 \\
70.2 \\
72.0 \\
71.0 \\
71.0 \\
70.0 \\
70.0 \\
70.0 \\
69.0 \\
68.0 \\
69.0 \\
69.0 \\
70.0 \\
74.0 \\
76.0 \\
77.0 \\
75.0 \\
72.5 \\
72.5 \\
70.0 \\
72.0\end{array}$ & $\begin{array}{l}2.00 \\
1.39 \\
1.39 \\
1.39 \\
0.86 \\
1.23 \\
1.53 \\
1.53 \\
1.06 \\
0.75 \\
0.75 \\
1.56 \\
1.21 \\
1.21 \\
1.21 \\
1.41 \\
1.41 \\
0.80 \\
0.80 \\
0.70 \\
0.70 \\
0.70 \\
1.28 \\
2.41 \\
2.30 \\
1.62 \\
0.93 \\
0.93 \\
0.93 \\
1.19 \\
0.68\end{array}$ & $\begin{array}{l}48.1 \\
33.3 \\
33.3 \\
33.3 \\
20.7 \\
36.8 \\
36.8 \\
36.8 \\
25.5 \\
17.9 \\
17.9 \\
37.5 \\
29.0 \\
29.0 \\
29.0 \\
33.9 \\
33.9 \\
19.2 \\
19.2 \\
16.8 \\
16.8 \\
16.8 \\
30.7 \\
57.9 \\
55.2 \\
38.9 \\
22.2 \\
22.2 \\
22.2 \\
28.6 \\
16.3\end{array}$ & $\begin{array}{l}0.07 \\
0.58 \\
0.58 \\
0.59 \\
0.00 \\
0.01 \\
0.01 \\
0.01 \\
0.08 \\
0.83 \\
0.84 \\
0.01 \\
0.11 \\
0.12 \\
0.12 \\
0.01 \\
0.01 \\
0.03 \\
0.03 \\
0.22 \\
0.22 \\
0.23 \\
0.18 \\
0.01 \\
0.08 \\
0.00 \\
0.06 \\
0.06 \\
0.06 \\
0.00 \\
0.03\end{array}$ & $\begin{array}{l}0.240 \\
0.192 \\
0.192 \\
0.192 \\
0.120 \\
0.264 \\
0.264 \\
0.264 \\
0.312 \\
0.168 \\
0.168 \\
0.240 \\
0.216 \\
0.216 \\
0.216 \\
0.240 \\
0.240 \\
0.216 \\
0.216 \\
0.168 \\
0.168 \\
0.168 \\
0.216 \\
0.264 \\
0.288 \\
0.240 \\
0.264 \\
0.264 \\
0.264 \\
0.192 \\
0.120\end{array}$ \\
\hline $\begin{array}{l}\text { MEAN } \\
\text { DAILY } \\
\text { VALUE }\end{array}$ & 79.4 & 88.1 & 71.2 & 1.22 & 29.5 & 0.17 & 0.219 \\
\hline $\begin{array}{l}\text { STANDARD } \\
\text { DEVIATION }\end{array}$ & 1.60 & 2.50 & 2.02 & 0.444 & 10.8 & 0.241 & 0.046 \\
\hline $\begin{array}{l}\text { EXTREME } \\
\text { DAILY } \\
\text { VALUE }\end{array}$ & NA & 92.0 & 68.0 & $\mathrm{NA}$ & $\begin{array}{l}\text { MAX } \\
57.9 \\
\text { MIN } \\
16.3\end{array}$ & $\begin{array}{l}\text { MAX } \\
0.84 \\
\text { MIN } \\
0.0\end{array}$ & $\begin{array}{l}\text { MAX } \\
0.312 \\
\text { MIN } \\
0.12\end{array}$ \\
\hline $\begin{array}{l}\text { TOTAL } \\
\text { FOR THE } \\
\text { MONTH }\end{array}$ & $\mathrm{NA}$ & NA & NA & $\mathrm{NA}$ & 915.7 & 5.31 & 6.820 \\
\hline
\end{tabular}


Table 1. (Continued)

VEGA BAJA AUGUST 1984

\begin{tabular}{|c|c|c|c|c|c|c|c|c|}
\hline DAY & $\begin{array}{c}\text { MEAN } \\
\left({ }^{\circ} \mathrm{F}\right)\end{array}$ & $\begin{array}{l}\text { TEMPERATURE } \\
\text { MAX } \\
\left({ }^{\circ}\right)\end{array}$ & $\begin{array}{l}\text { MIN } \\
(\mathrm{OF})\end{array}$ & & $\begin{array}{l}\text { WII } \\
\text { VELOC } \\
(\mathrm{MPH})\end{array}$ & $\begin{array}{l}\text { ID } \\
\text { (MPD) }\end{array}$ & $\begin{array}{l}\text { DAILY } \\
\text { RAINFALL } \\
\text { (IN/DAY) }\end{array}$ & $\begin{array}{c}\text { PAN } \\
\text { EVAPORATION } \\
\text { (IN/DAY) }\end{array}$ \\
\hline $\begin{array}{l}1 \\
2 \\
3 \\
4 \\
5 \\
6 \\
7 \\
8 \\
9 \\
10 \\
11 \\
12 \\
13 \\
14 \\
15 \\
16 \\
17 \\
18 \\
19 \\
20 \\
21 \\
22 \\
23 \\
24 \\
25 \\
26 \\
27 \\
28 \\
29 \\
30 \\
31\end{array}$ & $\begin{array}{l}79.0 \\
80.0 \\
77.9 \\
78.5 \\
78.5 \\
79.0 \\
78.3 \\
79.0 \\
80.8 \\
80.2 \\
80.2 \\
80.2 \\
79.5 \\
79.3 \\
81.5 \\
81.0 \\
81.3 \\
80.9 \\
80.9 \\
80.5 \\
81.3 \\
80.8 \\
80.3 \\
81.0 \\
80.4 \\
80.4 \\
79.8 \\
80.8 \\
80.8 \\
80.5 \\
79.5\end{array}$ & $\begin{array}{l}87.0 \\
88.0 \\
84.2 \\
86.1 \\
86.1 \\
88.0 \\
87.5 \\
89.0 \\
89.5 \\
91.0 \\
90.0 \\
90.0 \\
89.0 \\
89.0 \\
90.0 \\
89.0 \\
91.5 \\
92.8 \\
92.8 \\
94.0 \\
90.5 \\
89.8 \\
84.0 \\
89.0 \\
88.8 \\
88.8 \\
88.5 \\
86.0 \\
91.0 \\
89.0 \\
89.0\end{array}$ & $\begin{array}{l}71.0 \\
72.0 \\
71.5 \\
71.3 \\
71.3 \\
70.0 \\
69.0 \\
69.0 \\
72.0 \\
71.0 \\
71.0 \\
71.0 \\
70.0 \\
69.5 \\
73.0 \\
73.0 \\
71.0 \\
69.0 \\
69.0 \\
67.0 \\
72.0 \\
71.8 \\
71.5 \\
73.0 \\
72.0 \\
72.0 \\
71.0 \\
75.5 \\
70.5 \\
72.0 \\
70.0\end{array}$ & , & $\begin{array}{l}1.84 \\
1.84 \\
1.01 \\
1.01 \\
1.01 \\
0.79 \\
0.70 \\
1.27 \\
1.42 \\
1.32 \\
1.32 \\
1.32 \\
1.10 \\
1.09 \\
0.78 \\
0.50 \\
0.85 \\
0.85 \\
0.85 \\
0.86 \\
0.88 \\
0.88 \\
1.51 \\
1.14 \\
1.14 \\
1.14 \\
0.81 \\
1.09 \\
1.04 \\
0.73 \\
0.58\end{array}$ & $\begin{array}{l}44.2 \\
44.2 \\
24.2 \\
24.2 \\
24.2 \\
18.9 \\
16.7 \\
30.6 \\
34.0 \\
31.7 \\
31.7 \\
31.7 \\
26.4 \\
26.1 \\
18.7 \\
11.9 \\
20.3 \\
20.3 \\
20.3 \\
20.6 \\
21.2 \\
21.2 \\
36.3 \\
27.3 \\
27.3 \\
27.3 \\
19.4 \\
26.1 \\
24.8 \\
17.4 \\
14.0\end{array}$ & $\begin{array}{l}0.56 \\
0.57 \\
0.28 \\
0.28 \\
0.29 \\
0.00 \\
0.00 \\
0.00 \\
0.00 \\
0.01 \\
0.02 \\
0.02 \\
0.00 \\
0.24 \\
0.00 \\
0.02 \\
0.09 \\
0.09 \\
0.09 \\
0.12 \\
0.22 \\
0.23 \\
0.00 \\
0.24 \\
0.24 \\
0.25 \\
0.01 \\
0.00 \\
0.06 \\
0.03 \\
0.02\end{array}$ & $\begin{array}{l}0.192 \\
0.192 \\
0.216 \\
0.216 \\
0.216 \\
0.240 \\
0.240 \\
0.240 \\
0.288 \\
0.248 \\
0.248 \\
0.248 \\
0.240 \\
0.192 \\
0.168 \\
0.048 \\
0.216 \\
0.216 \\
0.216 \\
0.264 \\
0.432 \\
0.432 \\
0.240 \\
0.168 \\
0.168 \\
0.168 \\
0.168 \\
0.240 \\
0.216 \\
0.264 \\
0.192\end{array}$ \\
\hline
\end{tabular}

MEAN

DAILY

VALUE

$80.1 \quad 89.0$

71.1

$1.05 \quad 25.3$

0.13

0.227

STANDARD

DEVIATION

0.960

2.25

1.58

$0.314 \quad 7.56$

0.155

0.069

EXTREME

DAILY

NA $\quad 94.0$

67.0

NA

MAX

MAX

${ }^{44.2}{ }^{2}$

0.57

11.9

MIN

MAX

0.432

MIN

0.048

TOTAL

FOR THE

NA NA

NA

NA $\quad 783.2$

3.97

7.130

MONTH 
Table 1. (Continued)

VEGA BAJA SEPTEMBER 1984

\begin{tabular}{|c|c|c|c|c|c|c|c|}
\hline DAY & $\begin{array}{l}\text { MEAN } \\
\left({ }^{\circ} \mathrm{F}\right)\end{array}$ & $\begin{array}{l}\text { EMPERA } \\
\text { MAX } \\
\left({ }^{\circ}\right)\end{array}$ & $\begin{array}{l}\text { MIN } \\
(\circ F)\end{array}$ & $\begin{array}{l}\text { WII } \\
\text { VELO } \\
\text { (MPH) }\end{array}$ & $\begin{array}{l}\text { JD } \\
\text { ITY } \\
\text { (MPD) }\end{array}$ & $\begin{array}{l}\text { DAILY } \\
\text { RAINFALI } \\
\text { (IN/DAY) }\end{array}$ & $\begin{array}{l}\text { EAN } \\
\text { EJAPCRATION } \\
\text { (IN/DAY }\end{array}$ \\
\hline $\begin{array}{l}1 \\
2 \\
3 \\
4 \\
5 \\
6 \\
7 \\
8 \\
9 \\
10 \\
11 \\
12 \\
13 \\
14 \\
15 \\
16 \\
17 \\
18 \\
19 \\
20 \\
21 \\
22 \\
23 \\
24 \\
25 \\
26 \\
27 \\
28 \\
29 \\
30\end{array}$ & $\begin{array}{l}79.3 \\
79.3 \\
79.3 \\
79.0 \\
80.0 \\
81.0 \\
80.8 \\
80.7 \\
80.7 \\
80.5 \\
79.3 \\
79.9 \\
80.5 \\
80.5 \\
80.5 \\
80.5 \\
80.5 \\
79.9 \\
79.3 \\
81.0 \\
78.5 \\
78.7 \\
78.7 \\
78.8 \\
80.0 \\
87.3 \\
87.3 \\
81.0 \\
81.0 \\
81.0\end{array}$ & $\begin{array}{l}89.0 \\
89.0 \\
89.0 \\
89.0 \\
89.5 \\
90.0 \\
92.0 \\
91.0 \\
91.0 \\
90.0 \\
89.5 \\
89.8 \\
90.0 \\
89.0 \\
89.3 \\
89.3 \\
89.5 \\
89.0 \\
88.5 \\
91.0 \\
84.0 \\
87.3 \\
87.3 \\
90.5 \\
90.0 \\
91.5 \\
91.5 \\
93.0 \\
92.3 \\
92.3\end{array}$ & $\begin{array}{l}69.5 \\
69.5 \\
69.5 \\
59.0 \\
70.5 \\
72.0 \\
69.5 \\
70.3 \\
70.3 \\
71.0 \\
69.0 \\
70.0 \\
71.0 \\
72.0 \\
71.8 \\
71.8 \\
71.5 \\
70.8 \\
70.0 \\
71.0 \\
73.0 \\
70.0 \\
70.0 \\
67.0 \\
70.0 \\
69.5 \\
69.5 \\
69.0 \\
69.8 \\
69.8\end{array}$ & $\begin{array}{l}0.58 \\
0.58 \\
0.58 \\
0.82 \\
0.82 \\
0.94 \\
1.01 \\
1.01 \\
1.01 \\
0.89 \\
0.34 \\
0.34 \\
0.38 \\
0.37 \\
0.37 \\
0.37 \\
0.13 \\
0.13 \\
0.47 \\
0.07 \\
0.33 \\
0.33 \\
0.33 \\
0.45 \\
0.74 \\
0.74 \\
0.74 \\
0.86 \\
0.86 \\
0.86\end{array}$ & $\begin{array}{r}14.0 \\
14.0 \\
14.0 \\
20.2 \\
20.2 \\
22.5 \\
24.3 \\
24.3 \\
24.3 \\
21.3 \\
8.1 \\
8.1 \\
9.1 \\
8.8 \\
8.8 \\
8.8 \\
3.0 \\
3.0 \\
11.4 \\
1.6 \\
7.8 \\
7.8 \\
7.8 \\
10.7 \\
17.7 \\
17.7 \\
17.7 \\
20.7 \\
20.7 \\
20.7\end{array}$ & $\begin{array}{l}0.03 \\
0.03 \\
0.03 \\
0.15 \\
0.15 \\
0.01 \\
0.01 \\
0.01 \\
0.01 \\
0.01 \\
0.14 \\
0.14 \\
0.31 \\
0.31 \\
0.32 \\
0.32 \\
0.60 \\
0.61 \\
0.02 \\
0.02 \\
0.05 \\
0.06 \\
0.06 \\
0.00 \\
0.00 \\
0.00 \\
0.00 \\
0.01 \\
0.01 \\
0.01\end{array}$ & $\begin{array}{l}0.192 \\
0.192 \\
0.192 \\
0.164 \\
0.164 \\
0.231 \\
0.236 \\
0.236 \\
0.236 \\
0.178 \\
0.204 \\
0.204 \\
0.230 \\
0.085 \\
0.085 \\
0.085 \\
0.099 \\
0.099 \\
0.113 \\
0.106 \\
0.156 \\
0.156 \\
0.156 \\
0.217 \\
0.204 \\
0.204 \\
0.204 \\
0.222 \\
0.222 \\
0.222\end{array}$ \\
\hline $\begin{array}{l}\text { MEAN } \\
\text { DAILY } \\
\text { VALUE }\end{array}$ & 80.5 & 89.8 & 70.2 & 0.61 & 14.3 & 0.12 & 0.176 \\
\hline $\begin{array}{l}\text { STANDARD } \\
\text { DEVIATION }\end{array}$ & 1.98 & 1.74 & 1.18 & 0.282 & 6.83 & 0.167 & 0.051 \\
\hline $\begin{array}{l}\text { EXTREME } \\
\text { DAILY } \\
\text { VALUE }\end{array}$ & $\mathrm{NA}$ & 93.0 & 67.0 & $\mathrm{NA}$ & $\begin{array}{r}\text { MAX } \\
24.3 \\
M I N \\
1.6\end{array}$ & $\begin{array}{l}\text { MAX } \\
0.61 \\
\text { MIN } \\
0.00\end{array}$ & $\begin{array}{l}\text { MAX } \\
0.236 \\
\text { MIN } \\
0.095\end{array}$ \\
\hline $\begin{array}{l}\text { TOTAL } \\
\text { FOR THE } \\
\text { MONTH }\end{array}$ & NA & NA & $\mathrm{NA}$ & NA & 419.10 & 3.42 & 5.297 \\
\hline
\end{tabular}


Table 1. (Continued)

VEGA BAJA OCTOBER 1984

\begin{tabular}{|c|c|c|c|c|c|c|c|}
\hline DAY & $\begin{array}{l}\text { MEAN } \\
(O F)\end{array}$ & $\begin{array}{c}\text { TEMPERAT } \\
\text { MAX } \\
(\circ \mathrm{F})\end{array}$ & $\begin{array}{l}\text { MIN } \\
(O F)\end{array}$ & $\begin{array}{l}\text { WI } \\
\text { VELO } \\
\text { (MPH) }\end{array}$ & $\begin{array}{l}\text { VD } \\
\text { ITY } \\
\text { (MPD) }\end{array}$ & $\begin{array}{l}\text { DAILY } \\
\text { RAINFALL } \\
\text { (IN/DAY) }\end{array}$ & $\begin{array}{c}\text { PAN } \\
\text { EVAPORATION } \\
\text { (IN/DAY) }\end{array}$ \\
\hline $\begin{array}{l}1 \\
2 \\
3 \\
4 \\
5 \\
6 \\
7 \\
8 \\
9 \\
10 \\
11 \\
12 \\
13 \\
14 \\
15 \\
16 \\
17 \\
18 \\
19 \\
20 \\
21 \\
22 \\
23 \\
24 \\
25 \\
26 \\
27 \\
28 \\
29 \\
30 \\
31\end{array}$ & $\begin{array}{l}81.0 \\
81.0 \\
81.5 \\
81.0 \\
81.0 \\
81.0 \\
81.0 \\
81.0 \\
80.5 \\
77.8 \\
78.6 \\
78.6 \\
78.6 \\
78.6 \\
78.6 \\
79.3 \\
77.0 \\
78.0 \\
78.5 \\
79.9 \\
79.9 \\
81.3 \\
83.0 \\
79.3 \\
80.6 \\
81.8 \\
80.8 \\
80.8 \\
79.8 \\
79.3 \\
76.5\end{array}$ & $\begin{array}{l}92.3 \\
91.5 \\
93.0 \\
92.5 \\
92.5 \\
92.5 \\
92.5 \\
92.0 \\
92.0 \\
83.5 \\
87.8 \\
87.8 \\
87.8 \\
87.8 \\
87.8 \\
93.0 \\
90.0 \\
88.0 \\
88.0 \\
90.3 \\
90.3 \\
92.5 \\
97.0 \\
90.5 \\
91.5 \\
92.5 \\
90.0 \\
90.0 \\
87.4 \\
86.5 \\
85.5\end{array}$ & $\begin{array}{l}69.8 \\
70.5 \\
70.0 \\
69.5 \\
69.5 \\
69.5 \\
69.5 \\
69.5 \\
69.0 \\
72.0 \\
68.8 \\
68.8 \\
68.8 \\
68.8 \\
68.8 \\
65.5 \\
64.0 \\
68.0 \\
69.0 \\
69.5 \\
69.5 \\
70.0 \\
69.0 \\
68.0 \\
69.5 \\
71.0 \\
71.5 \\
71.5 \\
72.0 \\
69.8 \\
67.5\end{array}$ & $\begin{array}{l}0.00 \\
0.71 \\
0.28 \\
0.28 \\
0.28 \\
0.28 \\
0.28 \\
0.28 \\
0.02 \\
0.60 \\
0.60 \\
0.60 \\
0.50 \\
0.60 \\
0.60 \\
0.60 \\
0.43 \\
0.64 \\
0.77 \\
0.77 \\
0.77 \\
0.57 \\
0.09 \\
0.48 \\
0.48 \\
1.09 \\
1.09 \\
1.09 \\
0.21 \\
0.44 \\
0.16\end{array}$ & $\begin{array}{r}0.0 \\
17.1 \\
6.9 \\
6.9 \\
6.9 \\
6.9 \\
6.9 \\
6.9 \\
0.5 \\
14.3 \\
14.3 \\
14.3 \\
14.3 \\
14.3 \\
14.3 \\
14.3 \\
10.2 \\
15.3 \\
18.5 \\
18.5 \\
18.5 \\
13.7 \\
2.1 \\
10.2 \\
10.2 \\
26.3 \\
26.3 \\
26.3 \\
5.1 \\
10.6 \\
3.9\end{array}$ & $\begin{array}{l}0.00 \\
0.00 \\
0.36 \\
0.36 \\
0.36 \\
0.36 \\
0.37 \\
0.38 \\
0.01 \\
0.01 \\
0.01 \\
0.01 \\
0.00 \\
0.00 \\
0.00 \\
0.01 \\
0.05 \\
0.03 \\
0.31 \\
0.32 \\
0.32 \\
0.33 \\
0.20 \\
0.00 \\
0.00 \\
0.29 \\
0.30 \\
0.30 \\
0.25 \\
0.04 \\
0.00\end{array}$ & $\begin{array}{l}0.166 \\
0.188 \\
0.104 \\
0.104 \\
0.104 \\
0.104 \\
0.104 \\
0.104 \\
0.184 \\
0.264 \\
0.264 \\
0.264 \\
0.264 \\
0.264 \\
0.264 \\
0.157 \\
0.130 \\
0.183 \\
0.176 \\
0.176 \\
0.176 \\
0.186 \\
0.338 \\
0.095 \\
0.095 \\
0.166 \\
0.166 \\
0.166 \\
0.186 \\
0.182 \\
0.131\end{array}$ \\
\hline $\begin{array}{l}\text { MEAN } \\
\text { DAILY } \\
\text { VALUE }\end{array}$ & 79.8 & 90.2 & 69.3 & 0.51 & 12.1 & 0.15 & 0.176 \\
\hline $\begin{array}{l}\text { STANDARD } \\
\text { DEVIATION }\end{array}$ & 1.48 & 3.51 & 1.61 & 0.271 & 6.53 & 0.159 & 0.063 \\
\hline $\begin{array}{l}\text { EXTREME } \\
\text { DAILY } \\
\text { VALUE }\end{array}$ & NA & 93.0 & 64.0 & NA & $\begin{array}{c}\text { MAX } \\
26.3 \\
\text { MIN } \\
0.5\end{array}$ & $\begin{array}{l}\text { MAY } \\
0.38 \\
\text { MIN } \\
0.00\end{array}$ & $\begin{array}{l}\text { MAX } \\
0.338 \\
\text { MIN } \\
0.095\end{array}$ \\
\hline $\begin{array}{l}\text { TOTAL } \\
\text { FOR THE } \\
\text { MONTH }\end{array}$ & $\mathrm{NA}$ & NA & $\mathrm{NA}$ & $\mathrm{NA}$ & 382.5 & 4.75 & 5.460 \\
\hline
\end{tabular}


Table 1. (Continued)

VEGA BAJA NOVEMBER 1984

\begin{tabular}{|c|c|c|c|c|c|c|c|}
\hline DAY & $\begin{array}{l}\text { MEAN } \\
\left({ }^{\circ} \mathrm{F}\right)\end{array}$ & $\begin{array}{c}\text { TEMPERA } \\
\text { MAX } \\
\left({ }^{\circ} \mathrm{F}\right)\end{array}$ & $\begin{array}{l}\text { MIN } \\
(\text { OF })\end{array}$ & \multicolumn{2}{|c|}{$\begin{array}{c}\text { WIND } \\
\text { VELOCITY }\end{array}$} & $\begin{array}{c}\text { DAILY } \\
\text { RAINFALI } \\
\text { (IN/DAY) }\end{array}$ & $\begin{array}{c}\text { PAN } \\
\text { EVAPORATION } \\
\text { (IN/DAY) }\end{array}$ \\
\hline $\begin{array}{l}1 \\
2 \\
3 \\
4 \\
5 \\
6 \\
7 \\
8 \\
9 \\
10 \\
11 \\
12 \\
13 \\
14 \\
15 \\
16 \\
17 \\
18 \\
19 \\
20 \\
21 \\
22 \\
23 \\
24 \\
25 \\
26 \\
27 \\
28 \\
29 \\
30\end{array}$ & $\begin{array}{l}78.3 \\
79.5 \\
79.1 \\
79.1 \\
78.6 \\
78.6 \\
78.6 \\
78.5 \\
80.4 \\
77.9 \\
77.9 \\
77.9 \\
75.3 \\
76.0 \\
77.0 \\
74.3 \\
75.1 \\
75.1 \\
75.8 \\
77.3 \\
76.5 \\
75.9 \\
75.3 \\
77.1 \\
77.1 \\
78.8 \\
77.3 \\
75.3 \\
76.5 \\
76.5\end{array}$ & $\begin{array}{l}85.5 \\
86.5 \\
86.0 \\
86.0 \\
85.5 \\
84.8 \\
84.8 \\
84.0 \\
85.8 \\
85.7 \\
85.7 \\
85.7 \\
85.5 \\
81.0 \\
83.0 \\
83.5 \\
84.5 \\
84.5 \\
85.5 \\
85.0 \\
86.0 \\
85.0 \\
84.0 \\
88.3 \\
88.3 \\
92.5 \\
85.0 \\
84.0 \\
84.0 \\
84.0\end{array}$ & $\begin{array}{l}71.0 \\
72.5 \\
72.1 \\
72.1 \\
71.7 \\
72.4 \\
72.4 \\
73.0 \\
75.0 \\
70.0 \\
70.0 \\
70.0 \\
65.0 \\
71.0 \\
71.0 \\
65.0 \\
65.5 \\
65.5 \\
66.0 \\
69.5 \\
67.0 \\
66.8 \\
66.5 \\
65.8 \\
65.8 \\
65.0 \\
69.5 \\
66.5 \\
65.0 \\
65.0\end{array}$ & $\begin{array}{l}0.72 \\
0.25 \\
0.25 \\
0.25 \\
1.07 \\
1.07 \\
1.02 \\
1.46 \\
0.73 \\
0.73 \\
0.73 \\
0.73 \\
0.58 \\
1.91 \\
1.94 \\
0.99 \\
0.99 \\
0.99 \\
1.00 \\
1.30 \\
0.54 \\
0.54 \\
0.45 \\
0.45 \\
0.45 \\
0.23 \\
0.41 \\
0.45 \\
0.68 \\
0.75\end{array}$ & $\begin{array}{r}17.3 \\
6.1 \\
6.1 \\
6.1 \\
25.7 \\
25.7 \\
25.7 \\
35.1 \\
17.6 \\
17.6 \\
17.6 \\
17.6 \\
13.9 \\
45.9 \\
46.5 \\
23.7 \\
23.7 \\
23.7 \\
24.1 \\
31.2 \\
12.3 \\
12.3 \\
10.7 \\
10.7 \\
10.7 \\
5.7 \\
9.8 \\
10.7 \\
16.3 \\
18.0\end{array}$ & $\begin{array}{l}0.08 \\
0.54 \\
0.54 \\
0.55 \\
0.33 \\
0.33 \\
0.32 \\
0.13 \\
0.05 \\
0.04 \\
0.04 \\
0.04 \\
1.10 \\
0.27 \\
0.10 \\
0.25 \\
0.26 \\
0.26 \\
0.00 \\
0.03 \\
0.08 \\
0.07 \\
0.06 \\
0.06 \\
0.06 \\
0.64 \\
0.01 \\
0.01 \\
0.01 \\
0.22\end{array}$ & $\begin{array}{l}0.102 \\
0.108 \\
0.108 \\
0.108 \\
0.057 \\
0.057 \\
0.057 \\
0.176 \\
0.175 \\
0.175 \\
0.175 \\
0.175 \\
0.075 \\
0.146 \\
0.375 \\
0.160 \\
0.160 \\
0.160 \\
0.144 \\
0.203 \\
0.113 \\
0.113 \\
0.102 \\
0.102 \\
0.102 \\
0.112 \\
0.153 \\
0.159 \\
0.146 \\
0.108\end{array}$ \\
\hline $\begin{array}{l}\text { MEAN } \\
\text { DAILY } \\
\text { VALUE }\end{array}$ & 77.2 & 85.3 & 68.8 & 0.79 & 18.9 & 0.22 & 0.137 \\
\hline $\begin{array}{l}\text { STANDARD } \\
\text { DEVIATION }\end{array}$ & 1.53 & 1.92 & 3.07 & 0.434 & 10.5 & 0.245 & 0.059 \\
\hline $\begin{array}{l}\text { EXTREME } \\
\text { DAILY } \\
\text { VALUE }\end{array}$ & $\mathrm{NA}$ & 92.5 & 65.0 & NA & $\begin{array}{l}\text { MAX } \\
46.5 \\
\text { MIN } \\
5.7\end{array}$ & $\begin{array}{l}\text { MAX } \\
1.10 \\
\text { MIN } \\
0.00\end{array}$ & $\begin{array}{l}\text { MAX } \\
0.375 \\
\text { MIN } \\
0.057\end{array}$ \\
\hline $\begin{array}{l}\text { TOTAL } \\
\text { FOR THE } \\
\text { MONTH }\end{array}$ & NA & NA & NA & NA & 550.0 & 6.60 & 4.106 \\
\hline
\end{tabular}


Table 1. (Continued)

VEGA BAJA DECEMBER 1984

\begin{tabular}{|c|c|c|c|c|c|c|c|}
\hline$D A Y$ & $\begin{array}{l}\text { MEAN } \\
(\mathrm{OF})\end{array}$ & $\begin{array}{c}\text { TEMPEFATISE } \\
\text { MAX } \\
(O F)\end{array}$ & $\begin{array}{l}\text { MIN } \\
\text { (OF) }\end{array}$ & $\begin{array}{r}\text { WI } \\
\text { VELC } \\
\text { (MPH) }\end{array}$ & $\begin{array}{l}\text { ND } \\
\text { CITY } \\
\text { (MPD: }\end{array}$ & $\begin{array}{l}\text { DAILY } \\
\text { RAINFALI } \\
\text { (IN/DAY) }\end{array}$ & $\begin{array}{l}\text { PAN } \\
\text { EVAPORATION } \\
\text { (IN/DAY) }\end{array}$ \\
\hline $\begin{array}{l}1 \\
2 \\
3 \\
4 \\
5 \\
5 \\
7 \\
8 \\
9 \\
10 \\
11 \\
12 \\
13 \\
14 \\
15 \\
16 \\
17 \\
18 \\
19 \\
20 \\
21 \\
22 \\
23 \\
24 \\
25 \\
26 \\
27 \\
28 \\
29 \\
30 \\
31\end{array}$ & $\begin{array}{l}75.3 \\
76.3 \\
76.0 \\
76.5 \\
76.5 \\
77.0 \\
74.3 \\
74.7 \\
74.7 \\
74.7 \\
75.0 \\
75.8 \\
74.3 \\
74.6 \\
74.6 \\
74.6 \\
74.6 \\
74.8 \\
73.2 \\
73.3 \\
71.8 \\
72.9 \\
72.9 \\
72.9 \\
72.9 \\
74.0 \\
74.0 \\
73.8 \\
73.8 \\
73.8 \\
73.5\end{array}$ & $\begin{array}{l}84.5 \\
84.5 \\
85.0 \\
84.5 \\
84.5 \\
84.0 \\
82.5 \\
83.3 \\
83.3 \\
83.3 \\
84.0 \\
84.5 \\
84.0 \\
83.8 \\
83.8 \\
83.8 \\
83.8 \\
83.5 \\
83.0 \\
83.0 \\
82.5 \\
83.3 \\
83.3 \\
83.3 \\
83.3 \\
84.0 \\
84.0 \\
84.0 \\
84.0 \\
84.0 \\
84.0\end{array}$ & $\begin{array}{l}56.0 \\
65.0 \\
67.0 \\
68.0 \\
68.0 \\
69.0 \\
66.0 \\
56.0 \\
66.0 \\
66.0 \\
56.0 \\
67.0 \\
64.5 \\
65.3 \\
65.3 \\
65.3 \\
65.3 \\
66.0 \\
63.5 \\
63.5 \\
61.0 \\
62.5 \\
62.5 \\
62.5 \\
52.5 \\
64.0 \\
64.0 \\
63.5 \\
63.5 \\
63.5 \\
63.0\end{array}$ & $\begin{array}{l}0.75 \\
0.75 \\
1.22 \\
1.22 \\
1.22 \\
0.54 \\
0.64 \\
0.64 \\
0.64 \\
0.64 \\
0.10 \\
0.42 \\
1.70 \\
1.70 \\
1.70 \\
1.70 \\
1.70 \\
0.91 \\
0.91 \\
0.91 \\
1.85 \\
1.85 \\
1.85 \\
1.85 \\
1.85 \\
1.77 \\
2.34 \\
2.34 \\
2.34 \\
2.34 \\
2.02\end{array}$ & $\begin{array}{l}18.0 \\
18.0 \\
29.4 \\
29.4 \\
29.4 \\
15.3 \\
15.3 \\
15.3 \\
15.3 \\
15.3 \\
2.3 \\
10.1 \\
40.7 \\
40.7 \\
40.7 \\
40.7 \\
40.7 \\
21.7 \\
21.7 \\
21.7 \\
44.4 \\
44.4 \\
44.4 \\
44.4 \\
44.4 \\
42.6 \\
56.2 \\
56.2 \\
56.2 \\
56.2 \\
48.6\end{array}$ & $\begin{array}{l}0.22 \\
0.21 \\
0.17 \\
0.18 \\
0.18 \\
0.01 \\
0.50 \\
0.49 \\
0.49 \\
0.49 \\
0.01 \\
0.01 \\
0.16 \\
0.16 \\
0.16 \\
0.16 \\
0.16 \\
0.07 \\
0.07 \\
0.07 \\
0.18 \\
0.18 \\
0.18 \\
0.18 \\
0.18 \\
0.26 \\
0.45 \\
0.46 \\
0.46 \\
0.46 \\
0.39\end{array}$ & $\begin{array}{l}0.108 \\
0.108 \\
0.167 \\
0.167 \\
0.167 \\
0.122 \\
0.152 \\
0.152 \\
0.152 \\
0.152 \\
0.153 \\
0.183 \\
0.146 \\
0.146 \\
0.146 \\
0.146 \\
0.146 \\
0.070 \\
0.070 \\
0.070 \\
0.151 \\
0.151 \\
0.151 \\
0.151 \\
0.151 \\
0.168 \\
0.176 \\
0.176 \\
0.176 \\
0.176 \\
0.192\end{array}$ \\
\hline $\begin{array}{l}\text { MEAN } \\
\text { DAILY } \\
\text { VALUE }\end{array}$ & 74.5 & 83.8 & 64.9 & 1.37 & 32.9 & 0.22 & 0.151 \\
\hline $\begin{array}{l}\text { STANDARD } \\
\text { DEVIATION }\end{array}$ & 1.25 & 0.580 & 1.87 & 0.639 & 15.4 & 0.159 & 0.031 \\
\hline $\begin{array}{l}\text { EXTREME } \\
\text { DAILY } \\
\text { VALUE }\end{array}$ & NA & 84.5 & 61.0 & NA & $\begin{array}{l}\text { MAX } \\
56.2 \\
\text { MIN } \\
2.3\end{array}$ & $\begin{array}{l}\text { MAX } \\
0.50 \\
\text { MIN } \\
0.01\end{array}$ & $\begin{array}{c}\text { MAX } \\
0.192 \\
\text { MIN } \\
0.070\end{array}$ \\
\hline $\begin{array}{l}\text { TOTAL } \\
\text { FOR THE } \\
\text { MONTH }\end{array}$ & $\mathrm{NA}$ & NA & $\mathrm{NA}$ & $\mathrm{NA}$ & 019.7 & 6.47 & 4.681 \\
\hline
\end{tabular}


Table 1. (Continued)

VEGA BAJA JANUARY 1985

\begin{tabular}{|c|c|c|c|c|c|c|c|}
\hline DAY & $\begin{array}{l}\text { MEAN } \\
\left({ }^{\circ} \mathrm{F}\right)\end{array}$ & $\begin{array}{l}\text { TEMPERATUKE } \\
\text { MAX } \\
(\circ \mathrm{F})\end{array}$ & $\begin{array}{l}\text { MIN } \\
(O F)\end{array}$ & $\begin{array}{l}\text { WII } \\
\text { VELO } \\
(\mathrm{MPH})\end{array}$ & $\begin{array}{l}\text { ND } \\
\text { CITY } \\
\text { (MPD) }\end{array}$ & $\begin{array}{c}\text { DAILY } \\
\text { RAINFALL } \\
\text { (IN/DAY) }\end{array}$ & $\begin{array}{c}\text { PAN } \\
\text { EVAPORATION } \\
\text { (IN/DAY) }\end{array}$ \\
\hline $\begin{array}{l}1 \\
2 \\
3 \\
4 \\
5 \\
6 \\
7 \\
8 \\
9 \\
10 \\
11 \\
12 \\
13 \\
14 \\
15 \\
16 \\
17 \\
18 \\
19 \\
20 \\
21 \\
22 \\
23 \\
24 \\
25 \\
26 \\
27 \\
28 \\
29 \\
30 \\
31\end{array}$ & $\begin{array}{l}73.6 \\
73.6 \\
73.7 \\
75.3 \\
74.2 \\
74.2 \\
73.0 \\
71.5 \\
72.0 \\
73.5 \\
72.3 \\
72.3 \\
72.3 \\
71.8 \\
71.5 \\
71.0 \\
71.3 \\
72.8 \\
73.4 \\
73.4 \\
73.4 \\
74.0 \\
72.3 \\
72.0 \\
71.8 \\
72.4 \\
72.4 \\
73.0 \\
73.0 \\
73.0 \\
73.3\end{array}$ & $\begin{array}{l}83.7 \\
83.7 \\
83.4 \\
81.5 \\
82.3 \\
82.3 \\
83.0 \\
82.0 \\
82.0 \\
80.0 \\
80.8 \\
80.8 \\
80.8 \\
81.5 \\
81.5 \\
81.0 \\
80.5 \\
83.0 \\
86.3 \\
86.3 \\
86.3 \\
89.5 \\
83.0 \\
83.5 \\
84.0 \\
85.3 \\
85.3 \\
86.5 \\
86.5 \\
83.5 \\
84.0\end{array}$ & $\begin{array}{l}63.5 \\
63.5 \\
64.0 \\
69.0 \\
66.0 \\
66.0 \\
63.0 \\
61.0 \\
62.0 \\
67.0 \\
64.5 \\
64.5 \\
64.5 \\
62.0 \\
61.5 \\
61.0 \\
62.0 \\
62.5 \\
60.5 \\
60.5 \\
60.5 \\
58.5 \\
61.5 \\
60.5 \\
59.5 \\
59.5 \\
59.5 \\
59.5 \\
59.5 \\
62.5 \\
62.5\end{array}$ & $\begin{array}{l}2.02 \\
2.02 \\
1.38 \\
0.86 \\
0.86 \\
0.86 \\
0.84 \\
0.80 \\
0.51 \\
0.38 \\
0.38 \\
0.38 \\
0.38 \\
0.77 \\
0.14 \\
0.38 \\
0.46 \\
0.69 \\
0.69 \\
0.69 \\
0.69 \\
0.61 \\
0.86 \\
0.86 \\
0.88 \\
0.88 \\
0.88 \\
0.91 \\
1.04 \\
0.46 \\
1.95\end{array}$ & $\begin{array}{r}48.6 \\
48.6 \\
33.0 \\
20.6 \\
20.6 \\
20.6 \\
20.3 \\
19.2 \\
12.2 \\
9.2 \\
9.2 \\
9.2 \\
9.2 \\
18.5 \\
3.3 \\
9.2 \\
11.0 \\
16.4 \\
16.4 \\
16.4 \\
16.4 \\
14.6 \\
20.5 \\
20.5 \\
21.1 \\
21.1 \\
21.1 \\
21.9 \\
24.9 \\
11.2 \\
46.8\end{array}$ & $\begin{array}{l}0.00 \\
0.00 \\
0.20 \\
0.03 \\
0.03 \\
0.03 \\
0.01 \\
0.02 \\
0.60 \\
0.19 \\
0.18 \\
0.18 \\
0.18 \\
0.01 \\
0.01 \\
0.01 \\
0.01 \\
0.01 \\
0.01 \\
0.01 \\
0.00 \\
0.02 \\
0.01 \\
0.01 \\
0.05 \\
0.04 \\
0.04 \\
0.01 \\
0.08 \\
0.01 \\
0.08\end{array}$ & $\begin{array}{l}0.192 \\
0.192 \\
0.128 \\
0.134 \\
0.134 \\
0.134 \\
0.073 \\
0.273 \\
0.109 \\
0.128 \\
0.128 \\
0.128 \\
0.128 \\
0.109 \\
0.153 \\
0.125 \\
0.134 \\
0.163 \\
0.163 \\
0.163 \\
0.163 \\
0.165 \\
0.171 \\
0.171 \\
0.158 \\
0.158 \\
0.158 \\
0.185 \\
0.170 \\
0.170 \\
0.203\end{array}$ \\
\hline $\begin{array}{l}\text { MEAN } \\
\text { DAILY } \\
\text { VALUE }\end{array}$ & 72.8 & 83.3 & 62.3 & 0.82 & 19.7 & 0.09 & 0.154 \\
\hline $\begin{array}{l}\text { STANDARD } \\
\text { DEVIATION }\end{array}$ & 0.960 & 2.22 & 2.45 & 0.456 & 11.0 & 0.118 & 0.035 \\
\hline $\begin{array}{l}\text { EXTREME } \\
\text { DAILY } \\
\text { VALUE }\end{array}$ & NA & 89.5 & 58.5 & NA & $\begin{array}{l}\text { MAX } \\
48.5 \\
\text { MIN } \\
3.3\end{array}$ & $\begin{array}{l}\text { MAX } \\
0.60 \\
\text { MIN } \\
0.0\end{array}$ & $\begin{array}{c}\text { MAX } \\
0.273 \\
\text { MIN } \\
0.073\end{array}$ \\
\hline $\begin{array}{l}\text { TOTAL } \\
\text { FOR THE } \\
\text { MONTH }\end{array}$ & NA & NA & NA & NA & 590.7 & 2.84 & 4.763 \\
\hline
\end{tabular}




\begin{tabular}{|c|c|c|c|c|c|c|c|}
\hline DAY & $\begin{array}{l}\text { MEAN } \\
\left({ }^{\circ} \mathrm{F}\right)\end{array}$ & $\begin{array}{l}\text { TEMPERATURE } \\
\text { MAX } \\
\left({ }^{\circ} \mathrm{F}\right)\end{array}$ & $\begin{array}{l}\text { MIN } \\
\left({ }^{\circ} \mathrm{F}\right)\end{array}$ & $\begin{array}{l}\text { NII } \\
\text { VELOO } \\
\text { (MEH) }\end{array}$ & $\begin{array}{l}\text { ND } \\
\text { CITY } \\
\text { (MED) }\end{array}$ & $\begin{array}{l}\text { DAILY } \\
\text { RAINFALL } \\
\text { (IN/DAY }\end{array}$ & $\begin{array}{c}\text { EAN } \\
\text { EVAFORATION } \\
\text { I IN } / \text { DAY }\end{array}$ \\
\hline $\begin{array}{l}1 \\
2 \\
3 \\
4 \\
5 \\
6 \\
7 \\
8 \\
9 \\
10 \\
11 \\
12 \\
13 \\
14 \\
15 \\
16 \\
17 \\
18 \\
19 \\
20 \\
21 \\
22 \\
23 \\
24 \\
25 \\
26 \\
27 \\
28\end{array}$ & $\begin{array}{l}73.3 \\
73.3 \\
73.3 \\
73.3 \\
73.3 \\
73.3 \\
73.3 \\
73.4 \\
73.4 \\
73.4 \\
73.4 \\
73.4 \\
73.4 \\
73.5 \\
74.7 \\
74.7 \\
74.7 \\
74.7 \\
75.8 \\
75.2 \\
74.5 \\
74.3 \\
74.3 \\
74.3 \\
74.0 \\
72.8 \\
73.2 \\
73.5\end{array}$ & $\begin{array}{l}85.5 \\
85.5 \\
85.5 \\
85.5 \\
85.5 \\
85.5 \\
87.0 \\
85.5 \\
85.5 \\
86.5 \\
86.5 \\
86.5 \\
86.5 \\
86.0 \\
87.3 \\
87.3 \\
87.3 \\
87.3 \\
88.5 \\
85.8 \\
83.0 \\
83.3 \\
83.3 \\
83.3 \\
83.5 \\
80.5 \\
81.3 \\
82.0\end{array}$ & $\begin{array}{l}61.0 \\
61.0 \\
61.0 \\
61.0 \\
61.0 \\
61.0 \\
59.5 \\
60.3 \\
60.3 \\
50.3 \\
60.3 \\
60.3 \\
60.3 \\
61.0 \\
62.0 \\
62.0 \\
62.0 \\
62.0 \\
63.0 \\
64.5 \\
66.0 \\
65.3 \\
65.3 \\
65.3 \\
64.5 \\
65.0 \\
65.0 \\
65.0\end{array}$ & $\begin{array}{l}1.95 \\
1.95 \\
1.95 \\
1.95 \\
1.95 \\
1.95 \\
1.24 \\
1.24 \\
1.24 \\
1.24 \\
1.24 \\
1.24 \\
1.24 \\
1.39 \\
1.39 \\
1.39 \\
1.39 \\
1.39 \\
1.03 \\
1.03 \\
1.64 \\
1.64 \\
1.64 \\
1.64 \\
0.51 \\
0.98 \\
0.98 \\
1.22\end{array}$ & $\begin{array}{l}46.8 \\
46.8 \\
46.8 \\
46.8 \\
46.8 \\
46.8 \\
29.7 \\
29.7 \\
29.7 \\
29.7 \\
29.7 \\
29.7 \\
29.7 \\
33.4 \\
33.4 \\
33.4 \\
33.4 \\
33.4 \\
24.7 \\
24.7 \\
39.4 \\
39.4 \\
39.4 \\
39.4 \\
12.2 \\
23.4 \\
23.4 \\
29.4\end{array}$ & $\begin{array}{l}0.06 \\
0.06 \\
0.05 \\
0.06 \\
0.06 \\
0.06 \\
0.02 \\
0.01 \\
0.01 \\
0.01 \\
0.01 \\
0.01 \\
0.01 \\
0.32 \\
0.32 \\
0.31 \\
0.31 \\
0.31 \\
0.08 \\
0.07 \\
0.22 \\
0.22 \\
0.21 \\
0.21 \\
0.15 \\
0.86 \\
0.85 \\
0.03\end{array}$ & $\begin{array}{l}0.202 \\
0.203 \\
0.202 \\
0.203 \\
0.203 \\
0.203 \\
0.202 \\
0.203 \\
0.203 \\
0.203 \\
0.203 \\
0.203 \\
0.203 \\
0.246 \\
0.246 \\
0.245 \\
0.246 \\
0.246 \\
0.118 \\
0.118 \\
0.202 \\
0.202 \\
0.202 \\
0.202 \\
0.176 \\
0.122 \\
0.122 \\
0.194\end{array}$ \\
\hline $\begin{array}{l}\text { MEAN } \\
\text { DAILY } \\
\text { VALUE }\end{array}$ & 73.8 & 85.3 & 62.3 & 1.42 & 34.0 & 0.19 & 0.197 \\
\hline $\begin{array}{l}\text { STANDARD } \\
\text { DEVIATION }\end{array}$ & 0.720 & 1.97 & 2.04 & 0.360 & 8.71 & 0.220 & 0.036 \\
\hline $\begin{array}{l}\text { EXTREME } \\
\text { DAILY } \\
\text { VALUE }\end{array}$ & NA & 88.5 & 59.5 & NA & $\begin{array}{l}\text { MAX } \\
46.8 \\
\text { MIN } \\
12.20\end{array}$ & $\begin{array}{l}\text { MAX } \\
0.86 \\
\text { MIN } \\
0.01\end{array}$ & $\begin{array}{c}\text { MAX } \\
0.246 \\
\text { MIN } \\
0.118\end{array}$ \\
\hline $\begin{array}{l}\text { TOTAL } \\
\text { FOR THE } \\
\text { MONTH }\end{array}$ & NA & $\mathrm{NA}$ & NA & $\mathrm{NA}$ & 951.1 & 5.36 & 5.527 \\
\hline
\end{tabular}


Table 1. (Continued)

VEGA BAJA MARCH 1985

\begin{tabular}{|c|c|c|c|c|c|c|c|}
\hline DAY & $\begin{array}{l}\text { MEAN } \\
\left({ }^{\circ} \mathrm{F}\right)\end{array}$ & $\begin{array}{l}\text { EMPERAT } \\
\text { MAX } \\
(O F)\end{array}$ & $\begin{array}{l}\text { MIN } \\
\left({ }^{\circ} \mathrm{F}\right)\end{array}$ & $\begin{array}{l}\text { WII } \\
\text { VELO } \\
(\mathrm{MPH})\end{array}$ & $\begin{array}{l}\text { VD } \\
\text { CITY } \\
(\text { MPD) }\end{array}$ & $\begin{array}{l}\text { DAILY } \\
\text { RAINFALL } \\
\text { (IN/DAY) }\end{array}$ & $\begin{array}{c}\text { PAN } \\
\text { EVAPORATION } \\
(\text { IN/DAY })\end{array}$ \\
\hline $\begin{array}{l}1 \\
2 \\
3 \\
4 \\
5 \\
6 \\
7 \\
8 \\
9 \\
10 \\
11 \\
12 \\
13 \\
14 \\
15 \\
16 \\
17 \\
18 \\
19 \\
20 \\
21 \\
22 \\
23 \\
24 \\
25 \\
26 \\
27 \\
28 \\
29 \\
30 \\
31\end{array}$ & $\begin{array}{l}73.5 \\
73.5 \\
73.5 \\
73.5 \\
73.3 \\
73.0 \\
72.9 \\
72.8 \\
72.9 \\
72.9 \\
73.0 \\
72.8 \\
72.8 \\
72.8 \\
72.5 \\
73.3 \\
73.3 \\
74.0 \\
74.3 \\
74.3 \\
74.5 \\
74.4 \\
74.4 \\
74.4 \\
74.4 \\
74.3 \\
74.7 \\
74.7 \\
75.0 \\
73.8 \\
73.8\end{array}$ & $\begin{array}{l}82.8 \\
82.8 \\
82.8 \\
83.5 \\
83.3 \\
83.0 \\
83.0 \\
83.0 \\
83.0 \\
83.0 \\
83.0 \\
83.3 \\
83.3 \\
83.3 \\
83.5 \\
85.3 \\
85.3 \\
87.0 \\
86.8 \\
86.8 \\
86.5 \\
86.5 \\
86.5 \\
86.5 \\
86.5 \\
86.5 \\
85.3 \\
85.3 \\
84.0 \\
84.0 \\
84.0\end{array}$ & $\begin{array}{l}64.3 \\
64.3 \\
64.3 \\
63.5 \\
63.3 \\
63.0 \\
62.8 \\
62.5 \\
62.8 \\
62.8 \\
63.0 \\
62.3 \\
62.3 \\
62.3 \\
61.5 \\
61.3 \\
61.3 \\
61.0 \\
61.8 \\
61.8 \\
62.5 \\
62.3 \\
62.3 \\
62.3 \\
62.3 \\
62.0 \\
64.0 \\
64.0 \\
66.0 \\
64.0 \\
64.0\end{array}$ & $\begin{array}{l}1.22 \\
1.22 \\
1.22 \\
1.19 \\
1.19 \\
1.15 \\
1.15 \\
1.15 \\
1.15 \\
1.15 \\
1.06 \\
1.06 \\
1.06 \\
1.06 \\
1.14 \\
1.14 \\
1.14 \\
0.75 \\
0.75 \\
0.75 \\
1.09 \\
1.09 \\
1.09 \\
1.09 \\
1.09 \\
2.24 \\
2.24 \\
2.24 \\
1.08 \\
1.08 \\
1.08\end{array}$ & $\begin{array}{l}29.4 \\
29.4 \\
29.4 \\
28.6 \\
28.6 \\
27.5 \\
27.5 \\
27.5 \\
27.5 \\
27.5 \\
25.5 \\
25.5 \\
25.5 \\
25.5 \\
27.4 \\
27.4 \\
27.4 \\
18.1 \\
18.1 \\
18.1 \\
26.2 \\
26.2 \\
26.2 \\
26.2 \\
26.2 \\
53.8 \\
53.8 \\
53.8 \\
25.9 \\
25.9 \\
25.9\end{array}$ & $\begin{array}{l}0.02 \\
0.02 \\
0.02 \\
0.46 \\
0.46 \\
0.44 \\
0.43 \\
0.06 \\
0.06 \\
0.05 \\
0.02 \\
0.02 \\
0.01 \\
0.01 \\
0.06 \\
0.06 \\
0.06 \\
0.02 \\
0.02 \\
0.01 \\
0.01 \\
0.01 \\
0.00 \\
0.00 \\
0.00 \\
0.28 \\
0.28 \\
0.28 \\
0.20 \\
0.20 \\
0.20\end{array}$ & $\begin{array}{l}0.194 \\
0.194 \\
0.194 \\
0.185 \\
0.185 \\
0.076 \\
0.076 \\
0.194 \\
0.194 \\
0.194 \\
0.194 \\
0.194 \\
0.194 \\
0.194 \\
0.178 \\
0.178 \\
0.178 \\
0.178 \\
0.178 \\
0.178 \\
0.195 \\
0.195 \\
0.195 \\
0.195 \\
0.195 \\
0.230 \\
0.230 \\
0.230 \\
0.192 \\
0.192 \\
0.192\end{array}$ \\
\hline $\begin{array}{l}\text { MEAN } \\
\text { DAILY } \\
\text { VALUE }\end{array}$ & 73.6 & 84.5 & 62.8 & 1.19 & 28.8 & 0.12 & 0.188 \\
\hline $\begin{array}{l}\text { STANDARD } \\
\text { DEVIATION }\end{array}$ & 0.710 & 1.54 & 1.10 & 0.361 & 8.68 & 0.152 & 0.032 \\
\hline $\begin{array}{l}\text { EXTREME } \\
\text { DAILY } \\
\text { VALUE }\end{array}$ & NA & 87.0 & 61.0 & NA & $\begin{array}{l}\operatorname{MAX} \\
53.8 \\
\text { MIN } \\
18.1\end{array}$ & $\begin{array}{l}\text { MAX } \\
0.46 \\
\text { MIN } \\
0.0\end{array}$ & $\begin{array}{l}\text { MAX } \\
0.230 \\
\text { MIN } \\
0.076\end{array}$ \\
\hline $\begin{array}{l}\text { TOTAL } \\
\text { FOR THE } \\
\text { MONTH }\end{array}$ & $\mathrm{NA}$ & NA & $\mathrm{NA}$ & $\mathrm{NA}$ & 891.5 & 3.77 & 5.816 \\
\hline
\end{tabular}


Table 1. (Continued)

VEGA BAJA APRIL 1985

\begin{tabular}{|c|c|c|c|c|c|c|c|}
\hline DAY & $\begin{array}{l}\text { MEAN } \\
(O F)\end{array}$ & $\begin{array}{c}\text { TEMFEA } \\
\text { MAX } \\
(0 \vec{\nabla})\end{array}$ & $\begin{array}{l}\text { MIN } \\
(\text { OF) }\end{array}$ & $\begin{array}{c}\text { NLI } \\
\text { VELO } \\
\text { MEHI }\end{array}$ & $\begin{array}{l}\text { ID } \\
\text { ITI } \\
\text { (MPD) }\end{array}$ & $\begin{array}{l}\text { DAILY } \\
\text { RAINFALL } \\
\text { (IN/DAY) }\end{array}$ & $\begin{array}{c}\text { PAN } \\
\text { EVAPORATION } \\
(\text { IN / DAY })\end{array}$ \\
\hline $\begin{array}{l}1 \\
2 \\
3 \\
4 \\
5 \\
6 \\
7 \\
8 \\
9 \\
10 \\
11 \\
12 \\
13 \\
14 \\
15 \\
16 \\
17 \\
18 \\
19 \\
20 \\
21 \\
22 \\
23 \\
24 \\
25 \\
26 \\
27 \\
28 \\
29 \\
30\end{array}$ & $\begin{array}{l}73.8 \\
74.6 \\
72.3 \\
73.4 \\
73.4 \\
73.4 \\
77.3 \\
75.5 \\
75.7 \\
75.8 \\
75.8 \\
75.4 \\
75.4 \\
75.4 \\
75.0 \\
75.7 \\
75.7 \\
75.7 \\
76.3 \\
77.3 \\
77.3 \\
78.3 \\
76.4 \\
74.5 \\
74.5 \\
74.4 \\
74.4 \\
74.4 \\
74.3 \\
74.0\end{array}$ & $\begin{array}{l}84.0 \\
84.3 \\
84.3 \\
84.5 \\
94.5 \\
84.5 \\
88.4 \\
84.5 \\
85.5 \\
86.5 \\
35.5 \\
85.3 \\
85.3 \\
85.3 \\
85.0 \\
87.0 \\
87.0 \\
87.0 \\
89.0 \\
89.0 \\
89.0 \\
89.0 \\
87.5 \\
84.0 \\
84.0 \\
84.3 \\
84.3 \\
84.3 \\
84.5 \\
85.0\end{array}$ & $\begin{array}{l}63.5 \\
63.3 \\
62.3 \\
53.8 \\
53.8 \\
63.8 \\
56.3 \\
56.5 \\
65.8 \\
55.0 \\
65.0 \\
65.0 \\
65.0 \\
65.0 \\
65.0 \\
64.3 \\
64.3 \\
54.3 \\
63.5 \\
65.5 \\
65.5 \\
67.5 \\
66.3 \\
65.0 \\
65.0 \\
64.5 \\
64.5 \\
64.5 \\
64.0 \\
63.0\end{array}$ & $\begin{array}{l}1.95 \\
0.95 \\
1.14 \\
1.14 \\
1.14 \\
1.14 \\
1.14 \\
1.22 \\
1.22 \\
1.27 \\
1.22 \\
1.22 \\
1.22 \\
1.22 \\
1.35 \\
1.35 \\
1.35 \\
1.35 \\
1.19 \\
1.19 \\
1.19 \\
0.54 \\
0.54 \\
0.16 \\
1.07 \\
1.07 \\
1.07 \\
1.07 \\
1.13 \\
1.78\end{array}$ & $\begin{array}{l}22.7 \\
22.7 \\
27.4 \\
27.4 \\
27.4 \\
27.4 \\
27.4 \\
29.3 \\
29.3 \\
30.4 \\
29.3 \\
29.3 \\
29.3 \\
29.3 \\
32.4 \\
32.4 \\
32.4 \\
32.4 \\
28.5 \\
28.5 \\
28.5 \\
13.0 \\
13.0 \\
4.0 \\
25.6 \\
25.6 \\
25.6 \\
25.6 \\
27.0 \\
42.6\end{array}$ & $\begin{array}{l}0.01 \\
0.00 \\
0.06 \\
0.60 \\
0.06 \\
0.06 \\
0.00 \\
0.01 \\
0.00 \\
0.01 \\
0.26 \\
0.26 \\
0.26 \\
0.26 \\
0.01 \\
0.00 \\
0.00 \\
0.00 \\
0.05 \\
0.05 \\
0.04 \\
1.16 \\
1.16 \\
0.02 \\
0.02 \\
0.01 \\
0.01 \\
0.01 \\
0.10 \\
0.03\end{array}$ & $\begin{array}{l}0.235 \\
0.235 \\
0.203 \\
0.203 \\
0.203 \\
0.203 \\
0.200 \\
0.216 \\
0.216 \\
0.201 \\
0.229 \\
0.229 \\
0.229 \\
0.229 \\
0.234 \\
0.234 \\
0.234 \\
0.234 \\
0.237 \\
0.237 \\
0.237 \\
0.124 \\
0.124 \\
0.192 \\
0.238 \\
0.238 \\
0.238 \\
0.238 \\
0.238 \\
0.271\end{array}$ \\
\hline $\begin{array}{l}\text { MEAN } \\
\text { DAILY } \\
\text { VALUE }\end{array}$ & 75.2 & 85.5 & 64.5 & 1.12 & 25.8 & 0.13 & 0.217 \\
\hline $\begin{array}{l}\text { STANDARD } \\
\text { DEVIATION }\end{array}$ & 1.32 & 1.71 & 1.87 & 0.283 & 6.77 & 0.299 & 0.031 \\
\hline $\begin{array}{l}\text { EXTREME } \\
\text { DAILY } \\
\text { VALUE }\end{array}$ & NA & 89.0 & 56.3 & NA & $\begin{array}{l}\text { MAX } \\
42.6 \\
\text { MIN } \\
4.0\end{array}$ & $\begin{array}{l}\text { MAX } \\
1.16 \\
\text { MIN } \\
0.0\end{array}$ & $\begin{array}{c}\text { MAX } \\
0.271 \\
\text { MIN } \\
0.124\end{array}$ \\
\hline $\begin{array}{l}\text { TOTAL } \\
\text { FOR THE } \\
\text { MONTH }\end{array}$ & NA & $\mathrm{NA}$ & NA & NA & 805.7 & 4.02 & 6.509 \\
\hline
\end{tabular}


Table 1. (Continued)

VEGA BAJA MAY 1985

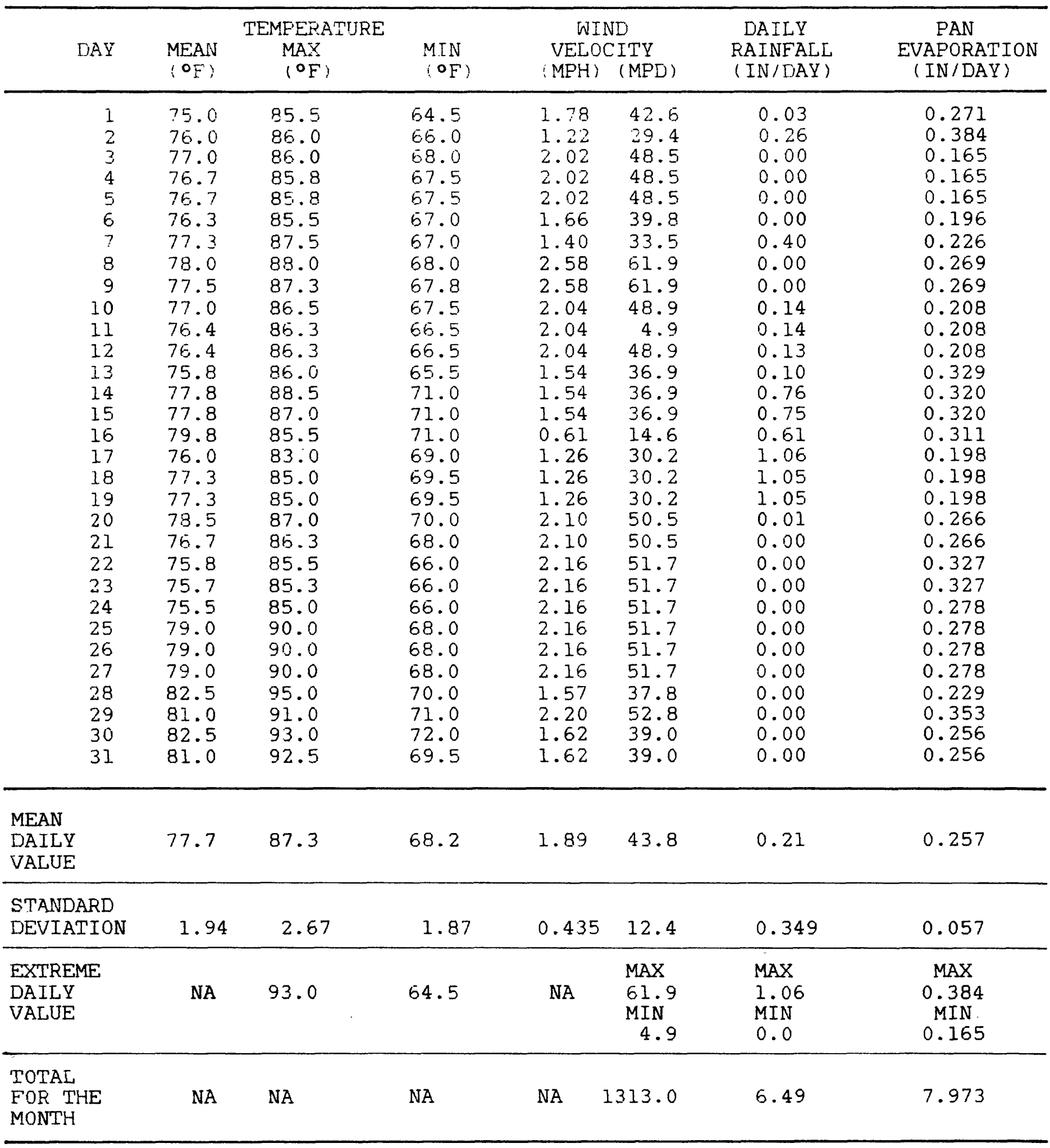


Table 1. (Continued)

VEGA BAJA JUNE 1985

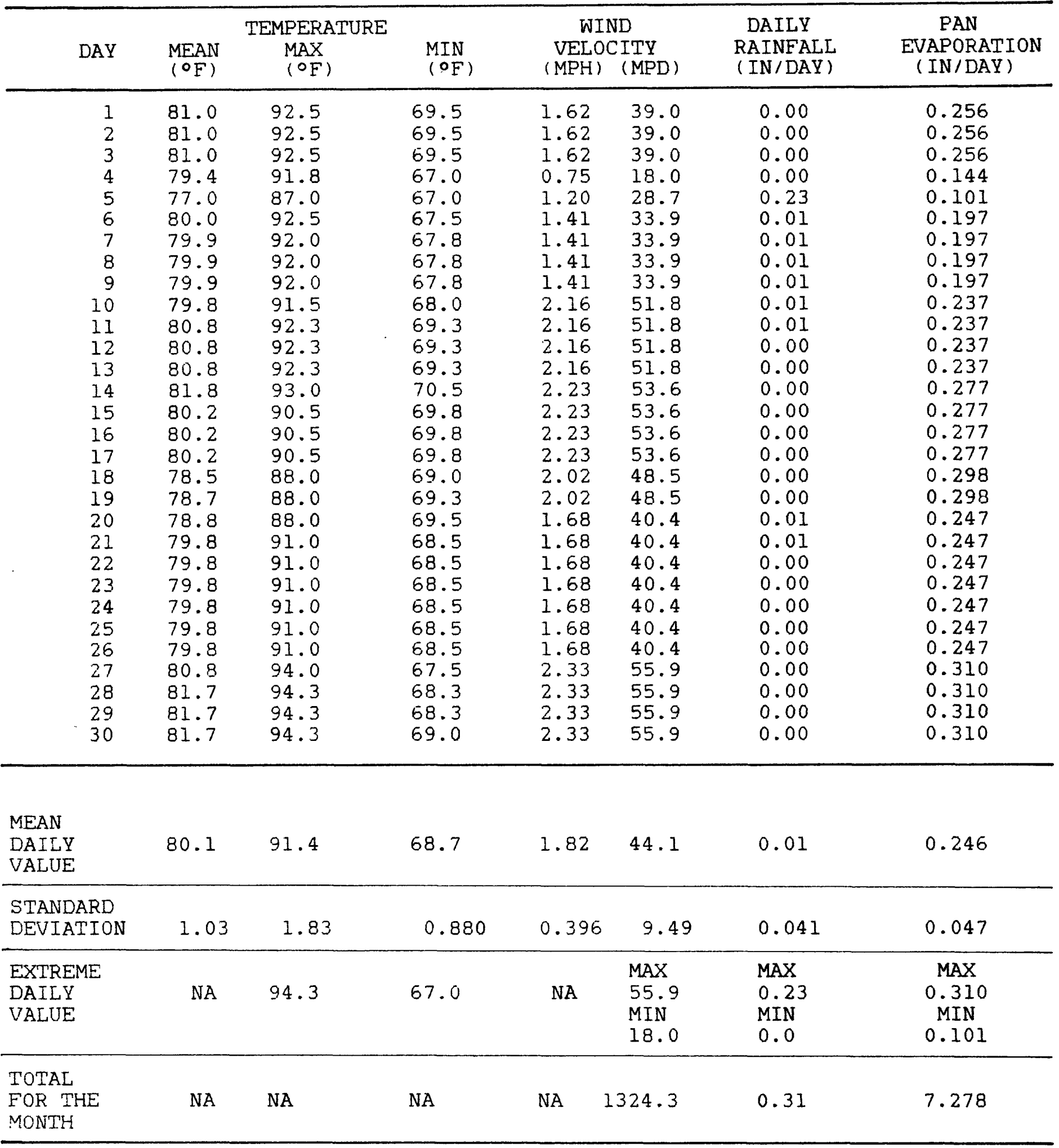


Table 1. (Continued)

VEGA BAJA JULY 1985

\begin{tabular}{|c|c|c|c|c|c|c|c|}
\hline DAY & $\begin{array}{l}\text { MEAN } \\
\left({ }^{\circ} \mathrm{F}\right)\end{array}$ & $\begin{array}{l}\text { TEMPERATURE } \\
\text { MAX } \\
\left({ }^{\circ} \mathrm{F}\right)\end{array}$ & $\begin{array}{l}\text { MIN } \\
\left({ }^{\circ} \mathrm{F}\right)\end{array}$ & $\begin{array}{l}\text { WI } \\
\text { VELO } \\
\text { (MPH) }\end{array}$ & $\begin{array}{l}\text { ND } \\
\text { CITY } \\
\quad(M P D)\end{array}$ & $\begin{array}{c}\text { DAILY } \\
\text { RAINFALI } \\
\text { (IN/DAY) }\end{array}$ & $\begin{array}{c}\text { PAN } \\
\text { EVAPORATION } \\
(\text { IN / DAY })\end{array}$ \\
\hline $\begin{array}{l}1 \\
2 \\
3 \\
4 \\
5 \\
6 \\
7 \\
8 \\
9 \\
10 \\
11 \\
12 \\
13 \\
14 \\
15 \\
16 \\
17 \\
18 \\
19 \\
20 \\
21 \\
22 \\
23 \\
24 \\
25 \\
26 \\
27 \\
28 \\
29 \\
30 \\
31\end{array}$ & $\begin{array}{l}82.5 \\
82.5 \\
82.5 \\
82.5 \\
82.5 \\
82.0 \\
82.0 \\
81.5 \\
81.3 \\
81.3 \\
81.3 \\
81.0 \\
81.0 \\
81.0 \\
81.0 \\
81.0 \\
81.0 \\
81.0 \\
81.0 \\
81.0 \\
81.0 \\
81.0 \\
81.0 \\
80.0 \\
80.0 \\
79.0 \\
80.0 \\
80.0 \\
81.0 \\
85.5 \\
80.5\end{array}$ & $\begin{array}{l}94.5 \\
94.8 \\
94.8 \\
94.8 \\
95.0 \\
92.3 \\
92.3 \\
89.5 \\
91.3 \\
91.3 \\
91.3 \\
93.0 \\
91.5 \\
91.5 \\
91.5 \\
90.0 \\
90.0 \\
90.0 \\
90.0 \\
91.0 \\
91.0 \\
91.0 \\
92.0 \\
90.0 \\
90.0 \\
88.0 \\
88.5 \\
88.5 \\
89.0 \\
89.5 \\
89.5\end{array}$ & $\begin{array}{l}70.5 \\
70.3 \\
70.3 \\
70.3 \\
70.0 \\
71.8 \\
71.8 \\
73.5 \\
71.3 \\
71.3 \\
71.3 \\
69.0 \\
70.5 \\
70.5 \\
70.5 \\
72.0 \\
72.0 \\
72.0 \\
72.0 \\
71.0 \\
71.0 \\
71.0 \\
70.0 \\
70.0 \\
70.0 \\
70.0 \\
71.5 \\
71.5 \\
73.0 \\
71.5 \\
71.5\end{array}$ & $\begin{array}{l}2.04 \\
2.04 \\
2.04 \\
2.04 \\
2.18 \\
2.18 \\
2.18 \\
1.61 \\
1.61 \\
1.61 \\
1.61 \\
1.53 \\
1.53 \\
1.53 \\
1.53 \\
1.21 \\
1.21 \\
1.21 \\
1.73 \\
1.73 \\
1.73 \\
1.73 \\
2.02 \\
2.02 \\
2.02 \\
1.22 \\
1.22 \\
1.22 \\
1.47 \\
1.47 \\
1.47\end{array}$ & $\begin{array}{l}49.1 \\
49.1 \\
49.1 \\
49.1 \\
52.4 \\
52.4 \\
52.4 \\
38.6 \\
38.6 \\
38.6 \\
38.6 \\
36.8 \\
36.8 \\
36.8 \\
36.8 \\
29.2 \\
29.2 \\
29.2 \\
41.6 \\
41.5 \\
41.6 \\
41.6 \\
48.6 \\
48.6 \\
48.6 \\
29.2 \\
29.2 \\
29.2 \\
35.4 \\
35.4 \\
35.4\end{array}$ & $\begin{array}{l}0.03 \\
0.03 \\
0.03 \\
0.02 \\
0.00 \\
0.00 \\
0.00 \\
0.02 \\
0.02 \\
0.02 \\
0.01 \\
0.12 \\
0.12 \\
0.12 \\
0.11 \\
0.06 \\
0.06 \\
0.06 \\
0.41 \\
0.41 \\
0.41 \\
0.41 \\
0.17 \\
0.17 \\
0.17 \\
0.05 \\
0.05 \\
0.05 \\
0.01 \\
0.00 \\
0.00\end{array}$ & $\begin{array}{l}0.245 \\
0.245 \\
0.245 \\
0.245 \\
0.280 \\
0.280 \\
0.280 \\
0.217 \\
0.217 \\
0.217 \\
0.217 \\
0.224 \\
0.224 \\
0.224 \\
0.224 \\
0.168 \\
0.168 \\
0.168 \\
0.306 \\
0.306 \\
0.306 \\
0.306 \\
0.285 \\
0.285 \\
0.285 \\
0.206 \\
0.206 \\
0.206 \\
0.287 \\
0.287 \\
0.287\end{array}$ \\
\hline $\begin{array}{l}\text { MEAN } \\
\text { DAILY } \\
\text { VALUE }\end{array}$ & 81.3 & 91.2 & 71.1 & 1.73 & 40.3 & 0.10 & 0.247 \\
\hline $\begin{array}{l}\text { STANDARD } \\
\text { DEVIATION }\end{array}$ & 1.13 & 1.95 & 0.950 & 0.324 & 7.72 & 0.129 & 0.042 \\
\hline $\begin{array}{l}\text { EXTREME } \\
\text { DAILY } \\
\text { VALUE }\end{array}$ & NA & 94.8 & 69.0 & NA & $\begin{array}{l}\text { MAX } \\
52.4 \\
\text { MIN } \\
29.2\end{array}$ & $\begin{array}{l}\text { MAX } \\
0.41 \\
\text { MIN } \\
0.0\end{array}$ & $\begin{array}{c}\text { MAX } \\
0.306 \\
\text { MIN } \\
0.168\end{array}$ \\
\hline $\begin{array}{l}\text { TOTAL } \\
\text { FOR THE } \\
\text { MONTH }\end{array}$ & NA & NA & NA & NA & 1248.8 & 3.14 & 7.646 \\
\hline
\end{tabular}


Table 1. (Continued)

VEGA BAJA AUGUST 1985

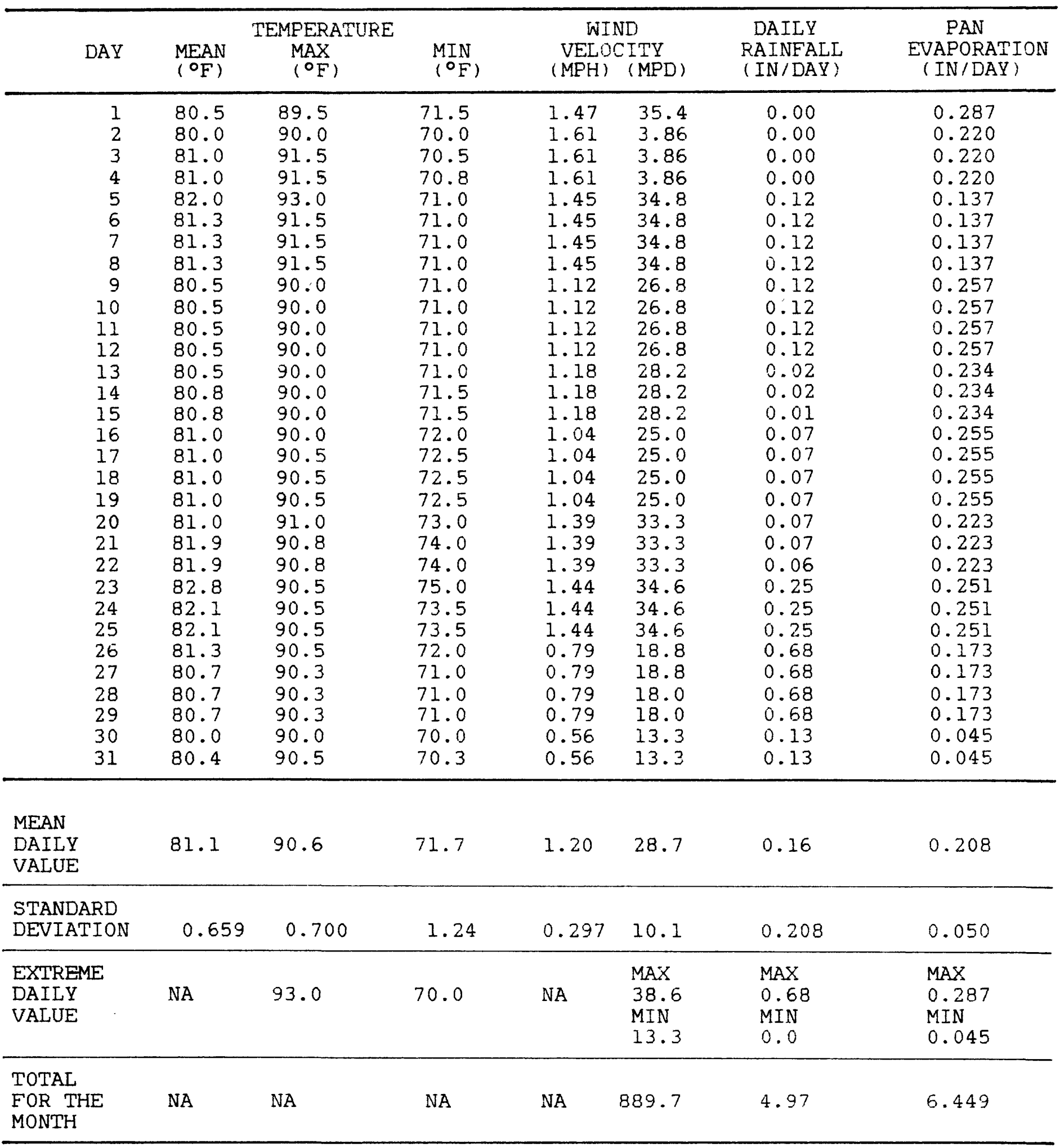


Tabie 1. (Continued)

VEGA BAJA SEPTEMBER 1985

\begin{tabular}{|c|c|c|c|c|c|c|c|}
\hline DAY & $\begin{array}{l}\text { MEAN } \\
\left({ }^{\circ} \mathrm{F}\right)\end{array}$ & $\begin{array}{c}\text { EMPERA } \\
\text { MAX } \\
\left({ }^{\circ} \mathrm{F}\right)\end{array}$ & $\begin{array}{l}\text { MIN } \\
(O F)\end{array}$ & $\begin{array}{r}\text { INI } \\
\text { VELO } \\
(M P H)\end{array}$ & $\begin{array}{l}\text { ND } \\
\text { (MPD) }\end{array}$ & $\begin{array}{c}\text { DAILY } \\
\text { RAINFALE } \\
\text { (IN/DAY) }\end{array}$ & $\begin{array}{c}\text { PAN } \\
\text { EVAPORATION } \\
\text { (IN/DAY) }\end{array}$ \\
\hline $\begin{array}{l}1 \\
2 \\
3 \\
4 \\
5 \\
6 \\
7 \\
8 \\
9 \\
10 \\
11 \\
12 \\
13 \\
14 \\
15 \\
16 \\
17 \\
18 \\
19 \\
20 \\
21 \\
22 \\
23 \\
24 \\
25 \\
26 \\
27 \\
28 \\
29 \\
30\end{array}$ & $\begin{array}{l}80.4 \\
80.4 \\
80.8 \\
80.9 \\
80.9 \\
81.0 \\
80.3 \\
80.3 \\
79.5 \\
80.0 \\
80.0 \\
80.0 \\
80.0 \\
80.0 \\
80.0 \\
80.0 \\
80.5 \\
80.4 \\
80.4 \\
80.3 \\
80.9 \\
80.4 \\
80.5 \\
80.3 \\
80.5 \\
80.5 \\
80.5 \\
80.5 \\
80.5 \\
80.5\end{array}$ & $\begin{array}{l}90.5 \\
90.5 \\
91.0 \\
90.5 \\
90.5 \\
90.0 \\
89.3 \\
89.3 \\
88.5 \\
89.8 \\
89.8 \\
89.8 \\
89.8 \\
89.8 \\
89.8 \\
89.8 \\
91.0 \\
91.0 \\
91.0 \\
91.0 \\
91.5 \\
91.5 \\
92.0 \\
87.5 \\
89.0 \\
89.0 \\
89.0 \\
89.0 \\
89.0 \\
89.0\end{array}$ & $\begin{array}{l}70.3 \\
70.3 \\
70.5 \\
71.3 \\
71.3 \\
72.0 \\
71.3 \\
71.3 \\
70.5 \\
70.3 \\
70.3 \\
70.3 \\
70.3 \\
70.3 \\
70.3 \\
70.3 \\
70.0 \\
59.8 \\
69.8 \\
69.5 \\
69.3 \\
59.3 \\
69.0 \\
73.0 \\
70.3 \\
70.3 \\
70.3 \\
70.3 \\
70.3 \\
70.3\end{array}$ & $\begin{array}{l}0.56 \\
0.56 \\
0.50 \\
0.50 \\
0.50 \\
0.46 \\
0.46 \\
0.46 \\
0.38 \\
0.38 \\
0.38 \\
0.38 \\
0.38 \\
0.38 \\
0.38 \\
0.38 \\
0.15 \\
0.15 \\
0.15 \\
0.21 \\
0.21 \\
0.21 \\
0.12 \\
0.23 \\
0.23 \\
0.23 \\
0.23 \\
0.23 \\
0.23 \\
0.23\end{array}$ & $\begin{array}{l}13.3 \\
13.3 \\
11.9 \\
11.9 \\
11.9 \\
10.9 \\
10.9 \\
10.9 \\
9.2 \\
9.2 \\
9.2 \\
9.2 \\
9.2 \\
9.2 \\
9.2 \\
9.2 \\
3.6 \\
3.6 \\
3.6 \\
5.1 \\
5.1 \\
5.1 \\
2.9 \\
5.5 \\
5.5 \\
5.5 \\
5.5 \\
5.5 \\
5.5 \\
5.5\end{array}$ & $\begin{array}{l}0.13 \\
0.13 \\
0.09 \\
0.08 \\
0.08 \\
0.17 \\
0.17 \\
0.16 \\
0.20 \\
0.19 \\
0.19 \\
0.19 \\
0.19 \\
0.19 \\
0.19 \\
0.19 \\
0.26 \\
0.26 \\
0.26 \\
0.23 \\
0.23 \\
0.23 \\
0.09 \\
0.20 \\
0.20 \\
0.20 \\
0.20 \\
0.20 \\
0.20 \\
0.20\end{array}$ & $\begin{array}{l}0.045 \\
0.045 \\
0.201 \\
0.201 \\
0.201 \\
0.169 \\
0.169 \\
0.169 \\
0.148 \\
0.148 \\
0.148 \\
0.148 \\
0.148 \\
0.148 \\
0.148 \\
0.148 \\
0.179 \\
0.179 \\
0.179 \\
0.174 \\
0.174 \\
0.174 \\
0.084 \\
0.134 \\
0.134 \\
0.134 \\
0.134 \\
0.134 \\
0.134 \\
0.134\end{array}$ \\
\hline $\begin{array}{l}\text { MEAN } \\
\text { DAILY } \\
\text { VALUE }\end{array}$ & 80.4 & 89.9 & 70.4 & 0.33 & 7.9 & 0.20 & 0.149 \\
\hline $\begin{array}{l}\text { STANDARD } \\
\text { DEVIATION }\end{array}$ & 0.333 & 1.01 & 0.792 & 0.131 & 3.12 & 0.052 & 0.037 \\
\hline $\begin{array}{l}\text { EXTREME } \\
\text { DAILY } \\
\text { VALUE }\end{array}$ & NA & 92.0 & 69.0 & NA & $\begin{array}{r}\text { MAX } \\
13.3 \\
\text { MIN } \\
2.9\end{array}$ & $\begin{array}{l}\text { MAX } \\
0.26 \\
\text { MIN } \\
0.09\end{array}$ & $\begin{array}{l}\text { MAX } \\
0.20 I \\
\text { MIN } \\
0.045\end{array}$ \\
\hline $\begin{array}{l}\text { TOTAL } \\
\text { FOR THE } \\
\text { MONTH }\end{array}$ & NA & NA & $\mathrm{NA}$ & NA & 237.0 & 5.00 & 4.460 \\
\hline
\end{tabular}


Table 1. (Continued)

VEGA BAJA OCTOBER 1985

\begin{tabular}{|c|c|c|c|c|c|c|c|}
\hline DAY & $\begin{array}{l}\text { MEAN } \\
(\circ \mathrm{F})\end{array}$ & $\begin{array}{l}\text { TEMPERATURE } \\
\text { MAX } \\
(\circ \mathrm{F})\end{array}$ & $\begin{array}{l}\text { MIN } \\
(\circ \mathrm{F})\end{array}$ & $\begin{array}{l}\text { WII } \\
\text { VELOC } \\
(M P H)\end{array}$ & $\begin{array}{l}\text { ND } \\
\text { CITY } \\
\text { (MPD) }\end{array}$ & $\begin{array}{l}\text { DAILY } \\
\text { RAINFALL } \\
\text { (IN/DAY) }\end{array}$ & $\begin{array}{c}\text { PAN } \\
\text { EVAPORATION } \\
\text { (IN/DAY) }\end{array}$ \\
\hline $\begin{array}{l}1 \\
2 \\
3 \\
4 \\
5 \\
6 \\
7 \\
8 \\
9 \\
10 \\
11 \\
12 \\
13 \\
14 \\
15 \\
16 \\
17 \\
18 \\
19 \\
20 \\
21 \\
22 \\
23 \\
24 \\
25 \\
26 \\
27 \\
28 \\
29 \\
30 \\
31\end{array}$ & $\begin{array}{l}80.5 \\
80.3 \\
80.0 \\
79.0 \\
79.0 \\
79.0 \\
79.0 \\
79.0 \\
79.0 \\
79.0 \\
79.0 \\
79.0 \\
79.0 \\
79.0 \\
79.0 \\
78.0 \\
78.5 \\
78.5 \\
78.5 \\
78.5 \\
78.5 \\
78.5 \\
78.5 \\
78.0 \\
79.2 \\
79.2 \\
79.2 \\
79.2 \\
80.3 \\
81.5 \\
81.0\end{array}$ & $\begin{array}{l}93.5 \\
90.8 \\
88.0 \\
89.0 \\
89.0 \\
89.0 \\
89.0 \\
89.0 \\
89.0 \\
89.0 \\
89.0 \\
89.0 \\
89.0 \\
89.0 \\
89.0 \\
90.0 \\
90.0 \\
90.0 \\
90.0 \\
90.0 \\
90.0 \\
90.0 \\
90.0 \\
90.0 \\
90.3 \\
90.3 \\
90.3 \\
90.3 \\
90.5 \\
91.0 \\
91.5\end{array}$ & $\begin{array}{l}67.5 \\
69.8 \\
72.0 \\
68.0 \\
68.0 \\
68.0 \\
68.0 \\
68.0 \\
68.0 \\
68.0 \\
68.0 \\
68.0 \\
68.0 \\
68.0 \\
68.0 \\
66.0 \\
67.0 \\
67.0 \\
67.0 \\
67.0 \\
67.0 \\
67.0 \\
67.0 \\
66.0 \\
68.0 \\
68.0 \\
68.0 \\
68.0 \\
70.0 \\
72.0 \\
70.5\end{array}$ & $\begin{array}{l}0.19 \\
0.19 \\
0.27 \\
0.27 \\
0.27 \\
0.27 \\
0.27 \\
0.27 \\
0.27 \\
0.27 \\
0.27 \\
0.27 \\
0.27 \\
0.27 \\
0.27 \\
0.28 \\
0.28 \\
0.28 \\
0.28 \\
0.28 \\
0.28 \\
0.28 \\
0.28 \\
0.08 \\
0.08 \\
0.08 \\
0.08 \\
0.08 \\
0.17 \\
0.17 \\
0.17\end{array}$ & $\begin{array}{l}4.6 \\
4.6 \\
6.5 \\
5.5 \\
6.5 \\
6.5 \\
6.5 \\
6.5 \\
6.5 \\
6.5 \\
6.5 \\
6.5 \\
5.5 \\
6.5 \\
6.5 \\
6.7 \\
6.7 \\
6.7 \\
6.7 \\
6.7 \\
6.7 \\
6.7 \\
6.7 \\
2.0 \\
2.0 \\
2.0 \\
2.0 \\
2.0 \\
4.0 \\
4.0 \\
4.0\end{array}$ & $\begin{array}{l}0.47 \\
0.47 \\
0.72 \\
0.71 \\
0.71 \\
0.71 \\
0.71 \\
0.71 \\
0.71 \\
0.71 \\
0.71 \\
0.71 \\
0.71 \\
0.71 \\
0.71 \\
0.23 \\
0.23 \\
0.37 \\
0.36 \\
0.36 \\
0.36 \\
0.36 \\
0.36 \\
0.48 \\
0.48 \\
0.47 \\
0.47 \\
0.47 \\
0.10 \\
0.28 \\
0.28\end{array}$ & $\begin{array}{l}0.189 \\
0.189 \\
0.176 \\
0.176 \\
0.176 \\
0.176 \\
0.176 \\
0.176 \\
0.176 \\
0.176 \\
0.176 \\
0.176 \\
0.176 \\
0.176 \\
0.176 \\
0.162 \\
0.162 \\
0.175 \\
0.175 \\
0.175 \\
0.175 \\
0.175 \\
0.175 \\
0.150 \\
0.150 \\
0.150 \\
0.150 \\
0.150 \\
0.114 \\
0.147 \\
0.147\end{array}$ \\
\hline $\begin{array}{l}\text { MEAN } \\
\text { DAILY } \\
\text { VALUE }\end{array}$ & 79.2 & 89.8 & 68.1 & 0.22 & 5.4 & 0.51 & 0.157 \\
\hline $\begin{array}{l}\text { STANDARD } \\
\text { DEVIATION }\end{array}$ & 0.805 & 1.01 & 1.40 & 0.074 & 1.74 & 0.188 & 0.019 \\
\hline $\begin{array}{l}\text { EXTREME } \\
\text { DAILY } \\
\text { VALUE }\end{array}$ & NA & 93.5 & 66.0 & NA & $\begin{array}{l}\text { MAX } \\
7.4 \\
\text { MIN } \\
2.0\end{array}$ & $\begin{array}{l}\text { MAX } \\
0.71 \\
\text { MIN } \\
0.10\end{array}$ & $\begin{array}{l}\text { MAX } \\
0.189 \\
\text { MIN } \\
0.114\end{array}$ \\
\hline $\begin{array}{l}\text { TOTAL } \\
\text { FOR THE } \\
\text { MONTH }\end{array}$ & NA & NA & NA & NA & 157.4 & 15.74 & 4.872 \\
\hline
\end{tabular}


Tabie 2. Weather data and statisticai analyses for the Manati weather station May 1983

\begin{tabular}{|c|c|c|c|c|c|c|c|}
\hline DAY & $\begin{array}{l}\text { MEAN } \\
(\mathrm{OF})\end{array}$ & $\begin{array}{l}\text { BMPERAI } \\
\text { MAX } \\
(\mathrm{OF})\end{array}$ & $\begin{array}{l}\text { MIN } \\
(O F)\end{array}$ & $\begin{array}{l}\text { WII } \\
\text { VELOC } \\
(\mathrm{MPH})\end{array}$ & $\begin{array}{l}\text { ND } \\
\text { CITY } \\
\text { (MPD) }\end{array}$ & $\begin{array}{l}\text { DAILY } \\
\text { RAINFALL } \\
\text { (IN/DAY) }\end{array}$ & $\begin{array}{c}\text { PAN } \\
\text { EVAPORATION } \\
\text { (IN/DAY) }\end{array}$ \\
\hline $\begin{array}{l}1 \\
2 \\
3 \\
4 \\
5 \\
6 \\
7 \\
8 \\
9 \\
10 \\
11 \\
12 \\
13 \\
14 \\
15 \\
16 \\
17 \\
18 \\
19 \\
20 \\
21 \\
22 \\
23 \\
24 \\
25 \\
26 \\
27 \\
28 \\
29 \\
30 \\
31\end{array}$ & $\begin{array}{l}78.0 \\
78.0 \\
78.0 \\
78.0 \\
78.0 \\
78.0 \\
78.0 \\
78.0 \\
78.0 \\
78.5 \\
78.0 \\
77.5 \\
80.0 \\
78.8 \\
78.8 \\
78.8 \\
77.5 \\
76.5 \\
75.5 \\
77.0 \\
78.3 \\
78.3 \\
77.5 \\
79.0 \\
77.5 \\
77.8 \\
78.0 \\
78.8 \\
78.8 \\
78.8 \\
79.5\end{array}$ & $\begin{array}{l}86.0 \\
86.0 \\
86.0 \\
86.0 \\
86.0 \\
86.0 \\
86.0 \\
86.0 \\
86.0 \\
87.0 \\
87.0 \\
85.0 \\
86.0 \\
84.0 \\
84.0 \\
84.0 \\
82.0 \\
85.0 \\
84.0 \\
84.0 \\
84.5 \\
84.5 \\
85.0 \\
86.0 \\
85.0 \\
85.5 \\
86.0 \\
87.5 \\
87.5 \\
87.5 \\
89.0\end{array}$ & $\begin{array}{l}70.0 \\
70.0 \\
70.0 \\
70.0 \\
70.0 \\
70.0 \\
70.0 \\
70.0 \\
70.0 \\
70.0 \\
69.0 \\
70.0 \\
74.0 \\
73.5 \\
73.5 \\
73.5 \\
73.0 \\
68.0 \\
67.0 \\
70.0 \\
70.0 \\
70.0 \\
70.0 \\
72.0 \\
70.0 \\
70.0 \\
70.0 \\
70.0 \\
70.0 \\
70.0 \\
70.0\end{array}$ & $\begin{array}{l}1.86 \\
1.86 \\
1.86 \\
1.86 \\
1.86 \\
1.86 \\
1.86 \\
1.86 \\
1.86 \\
2.00 \\
1.89 \\
1.19 \\
1.22 \\
1.22 \\
1.22 \\
1.22 \\
0.98 \\
1.13 \\
1.80 \\
1.70 \\
1.70 \\
1.70 \\
1.77 \\
1.52 \\
0.88 \\
0.88 \\
0.94 \\
0.94 \\
0.94 \\
0.94 \\
1.69\end{array}$ & $\begin{array}{l}44.6 \\
44.6 \\
44.6 \\
44.6 \\
44.6 \\
44.6 \\
44.6 \\
44.6 \\
44.6 \\
48.0 \\
45.4 \\
28.5 \\
29.2 \\
29.2 \\
29.2 \\
29.2 \\
23.5 \\
27.0 \\
43.3 \\
40.8 \\
40.8 \\
40.8 \\
42.5 \\
36.4 \\
21.0 \\
21.0 \\
22.5 \\
22.5 \\
22.5 \\
22.5 \\
39.3\end{array}$ & $\begin{array}{l}0.08 \\
0.08 \\
0.08 \\
0.08 \\
0.08 \\
0.08 \\
0.08 \\
0.10 \\
0.10 \\
0.00 \\
0.00 \\
0.38 \\
0.01 \\
0.01 \\
0.01 \\
0.02 \\
0.02 \\
0.05 \\
0.09 \\
0.14 \\
0.14 \\
0.14 \\
0.00 \\
0.00 \\
0.50 \\
0.50 \\
0.08 \\
0.05 \\
0.08 \\
0.08 \\
0.00\end{array}$ & $\begin{array}{l}0.024 \\
0.024 \\
0.024 \\
0.024 \\
0.024 \\
0.024 \\
0.024 \\
0.024 \\
0.024 \\
0.264 \\
0.264 \\
0.192 \\
0.120 \\
0.120 \\
0.120 \\
0.120 \\
0.048 \\
0.168 \\
0.264 \\
0.240 \\
0.240 \\
0.240 \\
0.288 \\
0.168 \\
0.168 \\
0.168 \\
0.192 \\
0.192 \\
0.192 \\
0.192 \\
0.312\end{array}$ \\
\hline $\begin{array}{l}\text { MEAN } \\
\text { DAILY } \\
\text { VALUE }\end{array}$ & 78.1 & 85.6 & 70.4 & 1.49 & 34.8 & 0.10 & 0.145 \\
\hline $\begin{array}{l}\text { STANDARD } \\
\text { DEVIATION }\end{array}$ & 0.837 & 1.37 & 1.54 & 0.390 & 9.42 & 0.127 & 0.094 \\
\hline $\begin{array}{l}\text { EXTREME } \\
\text { DAILY } \\
\text { VALUE }\end{array}$ & NA & 89.0 & 67.0 & $\mathrm{NA}$ & $\begin{array}{l}\text { MAX } \\
48.0 \\
\text { MIN } \\
21.0\end{array}$ & $\begin{array}{l}\text { MAX } \\
0.50 \\
\text { MIN } \\
0.00\end{array}$ & $\begin{array}{c}\text { MAX } \\
0.312 \\
\text { MIN } \\
0.024\end{array}$ \\
\hline $\begin{array}{l}\text { TOTAL } \\
\text { FOR THE } \\
\text { MONTH }\end{array}$ & NA & $\mathrm{NA}$ & NA & $\mathrm{NA}$ & 1106.5 & 3.19 & 4.488 \\
\hline
\end{tabular}


Table 2. (Continued)

MANATI JUNE 1983

\begin{tabular}{|c|c|c|c|c|c|c|c|}
\hline DAY & $\begin{array}{l}\text { MEAN } \\
(\mathrm{OF})\end{array}$ & $\begin{array}{l}\text { TEMPERATUFE } \\
\text { MAX } \\
(\circ \mathrm{O})\end{array}$ & $\begin{array}{l}\text { MIN } \\
(O F)\end{array}$ & $\begin{array}{l}\text { WII } \\
\text { VELOC } \\
(\mathrm{MPH})\end{array}$ & $\begin{array}{l}\text { ND } \\
\text { CITY } \\
\text { (MPD) }\end{array}$ & $\begin{array}{l}\text { DAILY } \\
\text { RAINFALL } \\
(\text { IN/DAY })\end{array}$ & $\begin{array}{c}\text { PAN } \\
\text { EVAPORATION } \\
(\text { IN/DAY })\end{array}$ \\
\hline $\begin{array}{l}1 \\
2 \\
3 \\
4 \\
5 \\
6 \\
7 \\
8 \\
9 \\
10 \\
11 \\
12 \\
13 \\
14 \\
15 \\
16 \\
17 \\
18 \\
19 \\
20 \\
21 \\
22 \\
23 \\
24 \\
25 \\
26 \\
27 \\
28 \\
29 \\
30\end{array}$ & $\begin{array}{l}79.0 \\
79.0 \\
79.0 \\
79.0 \\
79.0 \\
79.0 \\
80.5 \\
78.5 \\
77.5 \\
79.0 \\
79.8 \\
79.8 \\
80.5 \\
79.5 \\
82.0 \\
82.0 \\
81.5 \\
81.0 \\
81.0 \\
80.5 \\
82.5 \\
82.5 \\
83.0 \\
77.5 \\
78.3 \\
78.3 \\
79.0 \\
82.0 \\
80.5 \\
79.0\end{array}$ & $\begin{array}{l}87.0 \\
88.0 \\
88.5 \\
88.5 \\
88.5 \\
89.0 \\
89.0 \\
87.0 \\
85.0 \\
88.0 \\
89.0 \\
89.0 \\
90.0 \\
88.0 \\
92.0 \\
91.0 \\
90.0 \\
91.5 \\
91.5 \\
93.0 \\
93.0 \\
94.0 \\
92.0 \\
88.0 \\
90.0 \\
90.0 \\
92.0 \\
92.0 \\
91.0 \\
87.0\end{array}$ & $\begin{array}{l}71.0 \\
70.0 \\
69.5 \\
69.5 \\
69.5 \\
69.0 \\
72.0 \\
69.0 \\
69.0 \\
70.0 \\
70.5 \\
70.5 \\
71.0 \\
71.0 \\
72.0 \\
73.0 \\
73.0 \\
70.5 \\
70.5 \\
68.0 \\
72.0 \\
71.0 \\
74.0 \\
67.0 \\
66.5 \\
66.5 \\
66.0 \\
72.0 \\
70.0 \\
71.0\end{array}$ & $\begin{array}{l}1.62 \\
1.67 \\
1.67 \\
1.67 \\
1.67 \\
1.59 \\
1.78 \\
1.60 \\
1.69 \\
1.44 \\
1.44 \\
1.44 \\
0.69 \\
0.71 \\
0.39 \\
0.31 \\
0.84 \\
0.84 \\
0.84 \\
0.42 \\
0.73 \\
0.66 \\
0.47 \\
0.87 \\
0.87 \\
0.87 \\
0.55 \\
1.42 \\
1.58 \\
1.49\end{array}$ & $\begin{array}{l}38.9 \\
40.1 \\
40.1 \\
40.1 \\
40.1 \\
38.2 \\
42.8 \\
38.4 \\
40.6 \\
34.5 \\
34.5 \\
34.5 \\
16.5 \\
17.6 \\
9.2 \\
7.4 \\
20.2 \\
20.2 \\
20.2 \\
10.2 \\
17.4 \\
15.8 \\
11.2 \\
20.9 \\
20.9 \\
20.9 \\
13.3 \\
34.2 \\
38.0 \\
35.7\end{array}$ & $\begin{array}{l}0.00 \\
0.00 \\
0.00 \\
0.00 \\
0.00 \\
0.02 \\
0.01 \\
0.07 \\
0.02 \\
0.03 \\
0.03 \\
0.03 \\
0.62 \\
0.02 \\
0.21 \\
0.48 \\
0.17 \\
0.18 \\
0.18 \\
0.07 \\
0.00 \\
0.00 \\
0.00 \\
0.02 \\
0.02 \\
0.02 \\
0.81 \\
0.00 \\
0.00 \\
0.03\end{array}$ & $\begin{array}{l}0.288 \\
0.192 \\
0.192 \\
0.192 \\
0.192 \\
0.192 \\
0.264 \\
0.192 \\
0.264 \\
0.096 \\
0.096 \\
0.096 \\
0.768 \\
0.240 \\
0.360 \\
0.216 \\
0.1444 \\
0.144 \\
0.144 \\
0.096 \\
0.240 \\
0.672 \\
0.216 \\
0.264 \\
0.264 \\
0.264 \\
0.888 \\
0.192 \\
0.360 \\
0.024\end{array}$ \\
\hline $\begin{array}{l}\text { MEAN } \\
\text { DAILY } \\
\text { VALUE }\end{array}$ & 80.0 & 89.8 & 70.2 & 1.77 & 27.1 & 0.10 & 0.258 \\
\hline $\begin{array}{l}\text { STANDARD } \\
\text { DEVIATION }\end{array}$ & 1.51 & 2.10 & 1.93 & 0.803 & 11.6 & 0.193 & 0.189 \\
\hline $\begin{array}{l}\text { EXTREME } \\
\text { DAILY } \\
\text { VALUE }\end{array}$ & $\mathrm{NA}$ & 94.0 & 66.0 & $\mathrm{NA}$ & $\begin{array}{l}\text { MAX } \\
42.8 \\
\text { MIN } \\
7.4\end{array}$ & $\begin{array}{l}\text { MAX } \\
0.81 \\
\text { MIN } \\
0.00\end{array}$ & $\begin{array}{l}\text { MAX } \\
0.888 \\
\text { MIN } \\
0.024\end{array}$ \\
\hline $\begin{array}{l}\text { TOTAL } \\
\text { FOR THE } \\
\text { MONTH }\end{array}$ & NA & NA & NA & $\mathrm{NA}$ & 813.0 & 3.04 & 7.800 \\
\hline
\end{tabular}


Table 2. (Continued)

MANATÍ JULY 1983

\begin{tabular}{|c|c|c|c|c|c|c|c|}
\hline DAY & $\begin{array}{l}\text { MEAN } \\
(O F)\end{array}$ & $\begin{array}{l}\text { TEMPERATURE } \\
\text { MAX } \\
\left({ }^{\circ} \mathrm{F}\right)\end{array}$ & $\begin{array}{l}\text { MIN } \\
(O F)\end{array}$ & $\begin{array}{l}\text { WII } \\
\text { VELOC } \\
(\mathrm{MPH})\end{array}$ & $\begin{array}{l}\mathrm{ND} \\
\text { CITY } \\
(\mathrm{MPD})\end{array}$ & $\begin{array}{l}\text { DAILY } \\
\text { RAINFALL } \\
(\mathrm{IN} / \mathrm{DAY})\end{array}$ & $\begin{array}{c}\text { PAN } \\
\text { EVAPORATION } \\
(\text { IN / DAY })\end{array}$ \\
\hline $\begin{array}{l}1 \\
2 \\
3 \\
4 \\
5 \\
6 \\
7 \\
8 \\
9 \\
10 \\
11 \\
12 \\
13 \\
14 \\
15 \\
16 \\
17 \\
18 \\
19 \\
20 \\
21 \\
22 \\
23 \\
24 \\
25 \\
26 \\
27 \\
28 \\
29 \\
30 \\
31\end{array}$ & $\begin{array}{l}80.5 \\
80.0 \\
80.0 \\
80.0 \\
80.0 \\
79.5 \\
80.0 \\
80.5 \\
80.0 \\
80.0 \\
79.5 \\
79.5 \\
80.0 \\
81.5 \\
80.0 \\
79.8 \\
79.8 \\
79.5 \\
80.0 \\
80.5 \\
81.0 \\
81.0 \\
81.0 \\
81.0 \\
81.5 \\
80.0 \\
79.5 \\
79.5 \\
82.0 \\
80.8 \\
80.8\end{array}$ & $\begin{array}{l}87.0 \\
87.5 \\
87.5 \\
87.5 \\
87.5 \\
88.0 \\
89.0 \\
90.0 \\
90.0 \\
90.0 \\
90.0 \\
88.0 \\
89.0 \\
88.0 \\
88.0 \\
88.0 \\
88.0 \\
88.0 \\
88.0 \\
88.0 \\
90.0 \\
90.0 \\
90.0 \\
90.0 \\
92.0 \\
91.0 \\
89.0 \\
89.0 \\
91.0 \\
90.0 \\
90.0\end{array}$ & $\begin{array}{l}74.0 \\
72.5 \\
72.5 \\
72.5 \\
72.5 \\
71.0 \\
71.0 \\
71.0 \\
70.0 \\
70.0 \\
69.0 \\
71.0 \\
71.0 \\
75.0 \\
72.0 \\
71.5 \\
71.5 \\
71.0 \\
72.0 \\
73.0 \\
72.0 \\
72.0 \\
72.0 \\
72.0 \\
71.0 \\
71.0 \\
70.0 \\
71.0 \\
73.0 \\
71.5 \\
71.5\end{array}$ & $\begin{array}{l}0.94 \\
0.94 \\
0.94 \\
0.94 \\
0.94 \\
0.98 \\
0.98 \\
0.91 \\
0.91 \\
0.91 \\
0.61 \\
0.69 \\
1.45 \\
0.54 \\
0.93 \\
0.93 \\
0.93 \\
1.04 \\
1.20 \\
1.15 \\
1.15 \\
1.15 \\
1.15 \\
1.15 \\
0.85 \\
1.10 \\
1.12 \\
0.43 \\
0.75 \\
0.75 \\
0.75\end{array}$ & $\begin{array}{l}22.6 \\
22.6 \\
22.6 \\
22.6 \\
22.6 \\
23.6 \\
23.6 \\
21.9 \\
21.9 \\
21.9 \\
14.7 \\
16.6 \\
34.7 \\
12.9 \\
22.3 \\
22.3 \\
22.3 \\
24.9 \\
28.7 \\
27.5 \\
27.5 \\
27.5 \\
27.5 \\
27.5 \\
20.3 \\
26.4 \\
26.9 \\
10.3 \\
17.9 \\
17.9 \\
17.9\end{array}$ & $\begin{array}{l}0.50 \\
0.50 \\
0.50 \\
0.51 \\
0.51 \\
0.03 \\
0.03 \\
0.00 \\
0.00 \\
0.00 \\
0.13 \\
0.94 \\
0.00 \\
0.00 \\
0.11 \\
0.11 \\
0.12 \\
0.07 \\
0.00 \\
0.06 \\
0.06 \\
0.06 \\
0.06 \\
0.06 \\
0.00 \\
0.00 \\
0.00 \\
0.05 \\
0.21 \\
0.21 \\
0.22\end{array}$ & $\begin{array}{l}0.192 \\
0.192 \\
0.192 \\
0.192 \\
0.192 \\
0.168 \\
0.168 \\
0.264 \\
0.264 \\
0.264 \\
0.168 \\
0.192 \\
0.264 \\
0.240 \\
0.288 \\
0.288 \\
0.288 \\
0.072 \\
0.096 \\
0.240 \\
0.240 \\
0.240 \\
0.240 \\
0.240 \\
0.576 \\
0.240 \\
0.240 \\
0.240 \\
0.168 \\
0.168 \\
0.168\end{array}$ \\
\hline $\begin{array}{l}\text { MEAN } \\
\text { DAILY } \\
\text { VALUE }\end{array}$ & 80.3 & 89.0 & 71.6 & 0.97 & 22.6 & 0.16 & 0.213 \\
\hline $\begin{array}{l}\text { STANDARD } \\
\text { DEVIATION }\end{array}$ & 0.641 & 1.26 & 1.18 & 0.209 & 4.96 & 0.224 & 0.083 \\
\hline $\begin{array}{l}\text { EXTREME } \\
\text { DAILY } \\
\text { VALUE }\end{array}$ & NA & 92.0 & 59.0 & $\mathrm{NA}$ & $\begin{array}{l}\text { MAX } \\
34.7 \\
\text { MIN } \\
10.3\end{array}$ & $\begin{array}{l}\text { MAX } \\
0.94 \\
\text { MIN } \\
0.00\end{array}$ & $\begin{array}{c}\text { MAX } \\
0.960 \\
\text { MIN } \\
0.288\end{array}$ \\
\hline $\begin{array}{l}\text { TOTAL } \\
\text { FOR THE } \\
\text { MONTH }\end{array}$ & NA & NA & NA & $\mathrm{NA}$ & 700.4 & 5.05 & 6.600 \\
\hline
\end{tabular}


Table 2. (Continued)

MANATI AUGUST 1983

\begin{tabular}{|c|c|c|c|c|c|c|c|}
\hline DAY & $\begin{array}{l}\text { MEAN } \\
(O F)\end{array}$ & $\begin{array}{c}\text { TEMPERA } \\
\text { MAX } \\
(O F)\end{array}$ & $\begin{array}{l}\text { MIN } \\
(O \mathrm{~F})\end{array}$ & $\begin{array}{c}\text { WII } \\
\text { VELO } \\
(\mathrm{MPH})\end{array}$ & $\begin{array}{l}\text { ND } \\
\text { CITY } \\
\text { (MPD) }\end{array}$ & $\begin{array}{c}\text { DAILY } \\
\text { RAINFALL } \\
\text { (IN/UAY }\end{array}$ & $\begin{array}{l}\text { PAN } \\
\text { EVAPORATION } \\
\text { (IN/DAY) }\end{array}$ \\
\hline $\begin{array}{r}1 \\
2 \\
3 \\
4 \\
5 \\
6 \\
7 \\
8 \\
9 \\
10 \\
11 \\
12 \\
13 \\
14 \\
15 \\
16 \\
17 \\
18 \\
19 \\
20 \\
21 \\
22 \\
23 \\
24 \\
25 \\
26 \\
27 \\
28 \\
29 \\
30 \\
31\end{array}$ & $\begin{array}{l}79.5 \\
80.5 \\
80.5 \\
81.0 \\
80.5 \\
81.3 \\
81.3 \\
81.3 \\
82.0 \\
82.5 \\
82.5 \\
79.5 \\
80.3 \\
80.3 \\
81.0 \\
81.5 \\
81.5 \\
80.0 \\
82.0 \\
81.0 \\
81.0 \\
80.0 \\
80.0 \\
81.5 \\
80.0 \\
77.5 \\
78.3 \\
78.3 \\
79.0 \\
80.0 \\
79.0\end{array}$ & $\begin{array}{l}89.0 \\
90.0 \\
87.0 \\
90.0 \\
90.0 \\
91.0 \\
91.0 \\
91.0 \\
92.0 \\
94.0 \\
94.0 \\
89.0 \\
90.0 \\
90.0 \\
91.0 \\
91.0 \\
90.0 \\
90.0 \\
93.0 \\
91.5 \\
91.5 \\
90.0 \\
89.0 \\
89.0 \\
88.0 \\
84.0 \\
86.0 \\
86.0 \\
88.0 \\
90.0 \\
88.0\end{array}$ & $\begin{array}{l}70.0 \\
71.0 \\
74.0 \\
72.0 \\
71.0 \\
71.5 \\
71.5 \\
71.5 \\
72.0 \\
71.0 \\
71.0 \\
70.0 \\
70.5 \\
70.5 \\
71.0 \\
72.0 \\
73.0 \\
70.0 \\
71.0 \\
70.5 \\
70.5 \\
70.0 \\
71.0 \\
72.0 \\
72.0 \\
71.0 \\
70.5 \\
70.5 \\
70.0 \\
70.0 \\
70.0\end{array}$ & $\begin{array}{l}1.08 \\
0.59 \\
1.53 \\
1.59 \\
1.55 \\
1.55 \\
1.55 \\
1.55 \\
1.85 \\
1.75 \\
1.23 \\
1.34 \\
1.34 \\
1.34 \\
1.68 \\
0.44 \\
1.01 \\
1.79 \\
1.50 \\
1.50 \\
1.50 \\
1.96 \\
1.98 \\
1.92 \\
1.24 \\
1.69 \\
1.69 \\
1.69 \\
2.44 \\
1.78 \\
2.31\end{array}$ & $\begin{array}{l}25.9 \\
13.2 \\
36.6 \\
38.1 \\
37.1 \\
37.1 \\
37.1 \\
37.1 \\
44.4 \\
41.9 \\
29.5 \\
32.2 \\
32.2 \\
32.2 \\
40.2 \\
10.6 \\
24.1 \\
43.1 \\
36.0 \\
36.0 \\
36.0 \\
47.0 \\
47.5 \\
46.0 \\
29.7 \\
40.5 \\
40.5 \\
40.5 \\
58.5 \\
42.8 \\
55.4\end{array}$ & $\begin{array}{l}0.35 \\
0.00 \\
0.00 \\
0.12 \\
0.00 \\
0.00 \\
0.00 \\
0.00 \\
0.00 \\
0.18 \\
0.07 \\
0.26 \\
0.26 \\
0.27 \\
0.02 \\
0.39 \\
0.15 \\
0.00 \\
0.50 \\
0.50 \\
0.50 \\
0.00 \\
0.11 \\
0.06 \\
0.47 \\
0.32 \\
0.33 \\
0.33 \\
0.00 \\
0.00 \\
0.00\end{array}$ & $\begin{array}{l}0.168 \\
0.120 \\
0.254 \\
0.240 \\
0.216 \\
0.216 \\
0.216 \\
0.216 \\
0.312 \\
0.384 \\
0.240 \\
0.216 \\
0.216 \\
0.216 \\
0.312 \\
0.024 \\
0.144 \\
0.288 \\
0.192 \\
0.192 \\
0.192 \\
0.288 \\
0.168 \\
0.288 \\
0.192 \\
0.168 \\
0.168 \\
0.168 \\
0.240 \\
0.216 \\
0.264\end{array}$ \\
\hline $\begin{array}{l}\text { MEAN } \\
\text { DAILY } \\
\text { VALUE }\end{array}$ & 80.5 & 89.8 & 71.0 & 1.55 & 37.1 & 0.17 & 0.218 \\
\hline $\begin{array}{l}\text { STANDARD } \\
\text { DEVIATION }\end{array}$ & 1.20 & 2.17 & 0.938 & 0.409 & 9.88 & 0.179 & 0.066 \\
\hline $\begin{array}{l}\text { EXTREME } \\
\text { DAILY } \\
\text { VALUE }\end{array}$ & NA & 94.0 & 70.0 & NA & $\begin{array}{l}\text { MAX } \\
58.5 \\
\text { MIN } \\
10.6\end{array}$ & $\begin{array}{l}\text { MAX } \\
0.47 \\
\text { MIN } \\
0.00\end{array}$ & $\begin{array}{c}\text { MAX } \\
0.384 \\
\text { MIN } \\
0.024\end{array}$ \\
\hline $\begin{array}{l}\text { TOTAL } \\
\text { FOR THE } \\
\text { MONTH }\end{array}$ & $\mathrm{NA}$ & $\mathrm{NA}$ & $\mathrm{NA}$ & $\mathrm{NA}$ & 1150.1 & 5.23 & 6.744 \\
\hline
\end{tabular}


Table 2. (Continued)

MANATI SEPTEMBER 1983

\begin{tabular}{|c|c|c|c|c|c|c|c|}
\hline DAY & $\begin{array}{l}\text { MEAN } \\
\left({ }^{\circ} \mathrm{F}\right)\end{array}$ & $\begin{array}{c}\text { TEMPERA } \\
\text { MAX } \\
\left({ }^{\circ} \mathrm{F}\right)\end{array}$ & $\begin{array}{l}\text { MIN } \\
\left({ }^{\circ} \mathrm{F}\right)\end{array}$ & $\begin{array}{r}\text { WI } \\
\text { VELO } \\
(\mathrm{MPH})\end{array}$ & $\begin{array}{l}\text { ND } \\
\text { CITY } \\
\text { (MPD) }\end{array}$ & $\begin{array}{l}\text { DAILY } \\
\text { RAINFALL } \\
\text { ( IN/DAY) }\end{array}$ & $\begin{array}{c}\text { PAN } \\
\text { EVAPORATION } \\
(\text { IN/DAY })\end{array}$ \\
\hline $\begin{array}{l}1 \\
2 \\
3 \\
4 \\
5 \\
6 \\
7 \\
8 \\
9 \\
10 \\
11 \\
12 \\
13 \\
14 \\
15 \\
16 \\
17 \\
18 \\
19 \\
20 \\
21 \\
22 \\
23 \\
24 \\
25 \\
26 \\
27 \\
28 \\
29 \\
30\end{array}$ & $\begin{array}{l}82.0 \\
85.0 \\
82.8 \\
82.8 \\
82.8 \\
80.5 \\
79.0 \\
79.0 \\
80.0 \\
80.3 \\
80.3 \\
80.5 \\
80.5 \\
80.5 \\
80.5 \\
79.5 \\
79.3 \\
79.3 \\
79.0 \\
81.0 \\
78.5 \\
78.0 \\
78.5 \\
79.8 \\
79.8 \\
81.0 \\
80.5 \\
80.5 \\
80.5 \\
80.5\end{array}$ & $\begin{array}{l}91.0 \\
94.0 \\
92.5 \\
92.5 \\
92.5 \\
91.0 \\
88.0 \\
88.0 \\
91.0 \\
91.0 \\
91.0 \\
91.0 \\
90.0 \\
89.5 \\
89.0 \\
88.0 \\
88.0 \\
88.0 \\
88.0 \\
88.0 \\
86.0 \\
85.0 \\
87.0 \\
89.5 \\
89.5 \\
92.0 \\
91.0 \\
91.0 \\
91.0 \\
90.0\end{array}$ & $\begin{array}{l}73.0 \\
76.0 \\
73.0 \\
73.0 \\
73.0 \\
70.0 \\
70.0 \\
70.0 \\
71.0 \\
70.5 \\
70.5 \\
70.0 \\
71.0 \\
71.5 \\
72.0 \\
71.0 \\
70.5 \\
70.5 \\
70.0 \\
74.0 \\
71.0 \\
71.0 \\
70.0 \\
70.0 \\
70.0 \\
70.0 \\
70.0 \\
70.0 \\
70.0 \\
71.0\end{array}$ & $\begin{array}{l}2.56 \\
2.54 \\
2.54 \\
2.54 \\
2.54 \\
1.95 \\
1.63 \\
1.76 \\
2.72 \\
2.72 \\
2.72 \\
2.63 \\
4.67 \\
4.67 \\
1.88 \\
0.48 \\
0.49 \\
0.49 \\
2.47 \\
2.72 \\
1.06 \\
1.39 \\
1.14 \\
1.14 \\
1.14 \\
0.97 \\
1.31 \\
1.31 \\
1.47 \\
1.34\end{array}$ & $\begin{array}{r}61.4 \\
61.0 \\
61.0 \\
61.0 \\
61.0 \\
46.8 \\
39.1 \\
42.2 \\
65.3 \\
65.3 \\
65.3 \\
63.1 \\
112.1 \\
112.1 \\
45.1 \\
11.8 \\
11.8 \\
11.8 \\
59.3 \\
65.3 \\
25.4 \\
33.4 \\
27.4 \\
27.4 \\
27.4 \\
23.2 \\
31.4 \\
31.4 \\
35.3 \\
32.2\end{array}$ & $\begin{array}{l}0.02 \\
0.46 \\
0.00 \\
0.00 \\
0.00 \\
0.54 \\
0.00 \\
0.54 \\
0.08 \\
0.00 \\
0.00 \\
0.00 \\
0.47 \\
0.00 \\
0.13 \\
0.57 \\
0.00 \\
0.00 \\
0.42 \\
0.33 \\
0.05 \\
0.00 \\
0.28 \\
0.00 \\
0.00 \\
0.02 \\
0.00 \\
0.00 \\
0.00 \\
0.00\end{array}$ & $\begin{array}{l}0.384 \\
0.264 \\
0.264 \\
0.264 \\
0.264 \\
0.264 \\
0.312 \\
0.240 \\
0.240 \\
0.240 \\
0.240 \\
0.288 \\
0.192 \\
0.192 \\
0.192 \\
0.192 \\
0.192 \\
0.192 \\
0.192 \\
0.264 \\
0.096 \\
0.216 \\
0.168 \\
0.168 \\
0.168 \\
0.240 \\
0.168 \\
0.168 \\
0.264 \\
0.192\end{array}$ \\
\hline $\begin{array}{l}\text { MEAN } \\
\text { DAILY } \\
\text { VALUE }\end{array}$ & 80.5 & 89.8 & 71.1 & 1.97 & 47.2 & 0.13 & 0.202 \\
\hline $\begin{array}{l}\text { STANDARD } \\
\text { DEVIATION }\end{array}$ & 1.47 & 2.05 & 1.46 & 1.03 & 24.7 & 0.202 & 0.059 \\
\hline $\begin{array}{l}\text { EXTREME } \\
\text { DAILY } \\
\text { VALUE }\end{array}$ & $\mathrm{NA}$ & 94.0 & 70.0 & NA & $\begin{array}{l}\text { MAX } \\
112.1 \\
\text { MIN } \\
11.8\end{array}$ & $\begin{array}{l}\text { MAX } \\
0.57 \\
\text { MIN } \\
0.00\end{array}$ & $\begin{array}{c}\text { MAX } \\
0.384 \\
\text { MIN } \\
0.096\end{array}$ \\
\hline $\begin{array}{l}\text { TOTAL } \\
\text { FOR THE } \\
\text { MONTH }\end{array}$ & $\mathrm{NA}$ & NA & NA & NA & 1416.0 & 3.91 & 6.060 \\
\hline
\end{tabular}


Table 2. (Continued)

MANATÍ OCTOBER 1983

\begin{tabular}{|c|c|c|c|c|c|c|c|}
\hline$D A Y$ & $\begin{array}{l}\text { MEAN } \\
(O F)\end{array}$ & $\begin{array}{c}\text { TEMPEFA' } \\
\text { MAX } \\
\text { (OF) }\end{array}$ & $\begin{array}{l}\text { MIN } \\
\text { OF })\end{array}$ & $\begin{array}{l}\text { WII } \\
\text { VELO: } \\
\text { (MEH) }\end{array}$ & $\begin{array}{l}\text { ND } \\
\text { (MTY } \\
\text { (MED) }\end{array}$ & $\begin{array}{l}\text { DAILY } \\
\text { RAINFALL } \\
\text { (IN/DAY) }\end{array}$ & $\begin{array}{c}\text { PAN } \\
\text { EVAPORATION } \\
(\text { IN/DAY })\end{array}$ \\
\hline $\begin{array}{l}1 \\
2 \\
3 \\
4 \\
5 \\
5 \\
7 \\
8 \\
9 \\
10 \\
11 \\
12 \\
13 \\
14 \\
15 \\
16 \\
17 \\
18 \\
19 \\
20 \\
21 \\
22 \\
23 \\
24 \\
25 \\
26 \\
27 \\
28 \\
29 \\
30 \\
31\end{array}$ & $\begin{array}{l}80.3 \\
80.3 \\
80.0 \\
78.0 \\
78.0 \\
78.5 \\
80.8 \\
79.9 \\
79.9 \\
79.9 \\
79.0 \\
79.0 \\
81.5 \\
79.5 \\
78.8 \\
78.8 \\
78.0 \\
77.5 \\
77.8 \\
78.5 \\
80.0 \\
78.9 \\
78.9 \\
77.8 \\
78.0 \\
80.5 \\
80.0 \\
79.9 \\
79.9 \\
79.9 \\
79.8\end{array}$ & $\begin{array}{l}91.0 \\
91.0 \\
92.0 \\
86.0 \\
88.0 \\
89.0 \\
91.5 \\
90.0 \\
90.0 \\
90.0 \\
88.5 \\
88.0 \\
91.0 \\
88.0 \\
87.0 \\
87.0 \\
86.0 \\
86.0 \\
86.5 \\
87.0 \\
90.0 \\
89.3 \\
89.3 \\
88.5 \\
89.0 \\
91.0 \\
94.0 \\
93.3 \\
93.3 \\
93.3 \\
92.5\end{array}$ & $\begin{array}{l}69.5 \\
59.5 \\
68.0 \\
70.0 \\
68.0 \\
58.0 \\
69.0 \\
69.3 \\
69.3 \\
69.3 \\
69.5 \\
70.0 \\
72.0 \\
71.0 \\
70.5 \\
70.5 \\
70.0 \\
69.0 \\
69.0 \\
70.0 \\
70.0 \\
68.5 \\
68.5 \\
57.0 \\
67.0 \\
70.0 \\
66.0 \\
66.5 \\
66.5 \\
66.5 \\
67.0\end{array}$ & $\begin{array}{l}1.34 \\
1.34 \\
1.04 \\
1.42 \\
1.18 \\
1.32 \\
1.01 \\
1.01 \\
1.01 \\
1.01 \\
1.18 \\
1.14 \\
0.96 \\
0.69 \\
0.69 \\
0.69 \\
0.70 \\
0.70 \\
0.66 \\
0.53 \\
0.59 \\
0.59 \\
0.59 \\
0.89 \\
0.74 \\
0.30 \\
0.78 \\
0.78 \\
0.78 \\
0.78 \\
0.75\end{array}$ & $\begin{array}{l}32.2 \\
32.2 \\
25.1 \\
34.1 \\
28.3 \\
31.7 \\
24.2 \\
24.2 \\
24.2 \\
24.2 \\
28.3 \\
27.4 \\
23.0 \\
16.6 \\
16.6 \\
16.6 \\
16.8 \\
16.8 \\
15.8 \\
12.7 \\
14.2 \\
14.2 \\
14.2 \\
21.4 \\
17.8 \\
7.2 \\
18.7 \\
18.7 \\
18.7 \\
18.7 \\
18.0\end{array}$ & $\begin{array}{l}0.00 \\
0.00 \\
0.00 \\
0.00 \\
0.02 \\
0.00 \\
0.01 \\
0.01 \\
0.01 \\
0.01 \\
0.00 \\
0.28 \\
0.28 \\
0.13 \\
0.14 \\
0.14 \\
0.38 \\
0.40 \\
0.07 \\
0.12 \\
0.28 \\
0.28 \\
0.28 \\
0.02 \\
0.00 \\
0.03 \\
0.32 \\
0.32 \\
0.32 \\
0.32 \\
0.20\end{array}$ & $\begin{array}{l}0.192 \\
0.192 \\
0.216 \\
0.240 \\
0.192 \\
0.600 \\
0.192 \\
0.192 \\
0.192 \\
0.192 \\
0.048 \\
0.024 \\
0.024 \\
0.192 \\
0.192 \\
0.192 \\
0.336 \\
0.624 \\
0.024 \\
0.120 \\
0.240 \\
0.240 \\
0.240 \\
0.216 \\
0.168 \\
0.216 \\
0.168 \\
0.168 \\
0.168 \\
0.168 \\
0.312\end{array}$ \\
\hline $\begin{array}{l}\text { MEAN } \\
\text { DAILY } \\
\text { VALUE }\end{array}$ & 77.0 & 89.6 & 68.9 & 0.88 & 21.6 & 0.13 & 0.199 \\
\hline $\begin{array}{l}\text { STANDARD } \\
\text { DEVIATION }\end{array}$ & 2.49 & 2.32 & 1.47 & 0.270 & 6.51 & 0.140 & 0.128 \\
\hline $\begin{array}{l}\text { EXTREME } \\
\text { DAILY } \\
\text { VALUE }\end{array}$ & NA & 94.0 & 66.5 & $\mathrm{NA}$ & $\begin{array}{l}\text { MAX } \\
34.1 \\
\text { MIN } \\
7.2\end{array}$ & $\begin{array}{l}\text { MAX } \\
0.40 \\
\text { MIN } \\
0.00\end{array}$ & $\begin{array}{c}\text { MAX } \\
0.624 \\
\text { MIN } \\
0.024\end{array}$ \\
\hline $\begin{array}{l}\text { TOTAL } \\
\text { FOR THE } \\
\text { MONTH }\end{array}$ & NA & $\mathrm{NA}$ & NA & NA & 669.6 & 3.99 & 6.045 \\
\hline
\end{tabular}


Table 2. (Continued)

MANATI NOVEMBER 1983

\begin{tabular}{|c|c|c|c|c|c|c|c|}
\hline DAY & $\begin{array}{l}\text { MEAN } \\
\left({ }^{\circ} \mathrm{F}\right)\end{array}$ & $\begin{array}{c}\text { TEMPERA } \\
\text { MAX } \\
\left({ }^{\circ} \mathrm{F}\right)\end{array}$ & $\begin{array}{l}\text { MIN } \\
(O F)\end{array}$ & $\begin{array}{l}\text { WII } \\
\text { VELO } \\
\text { (MPH) }\end{array}$ & $\begin{array}{l}\text { ND } \\
\text { ITY } \\
\text { (MPD) }\end{array}$ & $\begin{array}{l}\text { DAILY } \\
\text { RAINFALL } \\
\text { (IN/DAY) }\end{array}$ & $\begin{array}{c}\text { PAN } \\
\text { EVAPORATION } \\
(\text { IN/DAY })\end{array}$ \\
\hline $\begin{array}{l}1 \\
2 \\
3 \\
4 \\
5 \\
6 \\
7 \\
8 \\
9 \\
10 \\
11 \\
12 \\
13 \\
14 \\
15 \\
16 \\
17 \\
18 \\
19 \\
20 \\
21 \\
22 \\
23 \\
24 \\
25 \\
26 \\
27 \\
28 \\
29 \\
30\end{array}$ & $\begin{array}{l}79.4 \\
79.0 \\
76.5 \\
77.0 \\
77.5 \\
77.5 \\
77.0 \\
77.0 \\
77.5 \\
77.5 \\
78.3 \\
78.3 \\
78.3 \\
78.3 \\
79.0 \\
81.0 \\
81.0 \\
83.0 \\
76.5 \\
76.8 \\
76.8 \\
77.0 \\
76.8 \\
76.5 \\
76.5 \\
76.5 \\
76.5 \\
76.5 \\
76.5 \\
76.5 \\
75.0 \\
76.0\end{array}$ & $\begin{array}{l}89.3 \\
86.0 \\
85.0 \\
85.0 \\
88.0 \\
88.0 \\
91.0 \\
86.0 \\
86.0 \\
89.0 \\
89.0 \\
89.0 \\
89.0 \\
92.0 \\
91.0 \\
91.0 \\
91.0 \\
87.0 \\
87.5 \\
87.5 \\
88.0 \\
88.0 \\
88.0 \\
88.0 \\
88.0 \\
88.0 \\
88.0 \\
88.0 \\
85.0 \\
84.0\end{array}$ & $\begin{array}{l}69.5 \\
72.0 \\
68.0 \\
69.0 \\
67.0 \\
67.0 \\
65.0 \\
69.0 \\
69.0 \\
67.5 \\
67.5 \\
67.5 \\
67.5 \\
66.0 \\
70.5 \\
70.5 \\
75.0 \\
65.0 \\
65.0 \\
66.0 \\
66.0 \\
65.5 \\
65.0 \\
65.0 \\
65.0 \\
65.0 \\
65.0 \\
65.0 \\
65.0 \\
68.0\end{array}$ & $\begin{array}{l}0.75 \\
0.59 \\
0.32 \\
0.57 \\
0.57 \\
0.57 \\
0.97 \\
1.09 \\
0.76 \\
0.76 \\
0.76 \\
0.76 \\
0.76 \\
0.86 \\
0.86 \\
0.86 \\
0.61 \\
1.15 \\
1.15 \\
1.15 \\
0.90 \\
0.90 \\
0.91 \\
0.91 \\
0.91 \\
0.91 \\
0.91 \\
1.14 \\
0.56 \\
0.67\end{array}$ & $\begin{array}{l}18.0 \\
14.2 \\
7.7 \\
13.7 \\
13.7 \\
13.7 \\
23.8 \\
26.2 \\
18.2 \\
18.2 \\
18.2 \\
18.2 \\
18.2 \\
20.6 \\
20.6 \\
20.6 \\
14.6 \\
27.6 \\
27.6 \\
27.6 \\
21.6 \\
21.6 \\
21.8 \\
21.8 \\
21.8 \\
21.8 \\
21.8 \\
27.4 \\
13.4 \\
20.9\end{array}$ & $\begin{array}{l}0.21 \\
0.02 \\
0.93 \\
0.38 \\
0.38 \\
0.39 \\
0.02 \\
0.01 \\
0.39 \\
0.39 \\
0.39 \\
0.39 \\
0.39 \\
0.00 \\
0.00 \\
0.01 \\
0.00 \\
0.02 \\
0.02 \\
0.03 \\
0.01 \\
0.01 \\
0.12 \\
0.12 \\
0.12 \\
0.12 \\
0.13 \\
0.03 \\
0.14 \\
0.01\end{array}$ & $\begin{array}{l}0.312 \\
0.216 \\
0.600 \\
0.528 \\
0.528 \\
0.528 \\
0.216 \\
0.192 \\
0.288 \\
0.288 \\
0.288 \\
0.288 \\
0.288 \\
0.192 \\
0.192 \\
0.192 \\
0.192 \\
0.192 \\
0.192 \\
0.192 \\
0.192 \\
0.192 \\
0.168 \\
0.168 \\
0.168 \\
0.168 \\
0.168 \\
0.192 \\
0.096 \\
0.168\end{array}$ \\
\hline $\begin{array}{l}\text { MEAN } \\
\text { DAILY } \\
\text { VALUE }\end{array}$ & 77.7 & 88.0 & 67.3 & 0.82 & 19.8 & 0.17 & 0.253 \\
\hline $\begin{array}{l}\text { STANDARD } \\
\text { DEVIATION }\end{array}$ & 1.66 & 1.95 & 2.38 & 0.202 & 4.83 & 0.209 & 0.125 \\
\hline $\begin{array}{l}\text { EXTREME } \\
\text { DAILY } \\
\text { VALUE }\end{array}$ & $\mathrm{NA}$ & 92.0 & 65.0 & NA & $\begin{array}{l}\text { MAX } \\
27.6 \\
\text { MIN } \\
7.7\end{array}$ & $\begin{array}{l}\text { MAX } \\
0.93 \\
\text { MIN } \\
0.00\end{array}$ & $\begin{array}{c}\text { MAX } \\
0.600 \\
\text { MIN } \\
0.096\end{array}$ \\
\hline $\begin{array}{l}\text { TOTAL } \\
\text { FOR THE } \\
\text { MONTH }\end{array}$ & NA & NA & $\mathrm{NA}$ & NA & 594.0 & 5.20 & 7.584 \\
\hline
\end{tabular}


Table 2. (Continued)

MANATI DECEMBER 1983

\begin{tabular}{|c|c|c|c|c|c|c|c|}
\hline DAY & $\begin{array}{l}\text { MEAN } \\
(\mathrm{OF})\end{array}$ & $\begin{array}{l}\text { TEMPERATURE } \\
\text { MAX } \\
(\circ \mathrm{F})\end{array}$ & $\begin{array}{l}\text { MIN } \\
(O F)\end{array}$ & $\begin{array}{r}\text { WI } \\
\text { VELO } \\
\text { (MPH) }\end{array}$ & $\begin{array}{l}\text { ND } \\
\text { CITY } \\
\text { (MPD) }\end{array}$ & $\begin{array}{l}\text { DAILY } \\
\text { RAINEALL } \\
\text { (IN/DAY) } \\
\end{array}$ & $\begin{array}{l}\text { PAN } \\
\text { EVAFORATION } \\
\text { (IN/DAY) }\end{array}$ \\
\hline $\begin{array}{l}1 \\
2 \\
3 \\
4 \\
5 \\
6 \\
7 \\
8 \\
9 \\
10 \\
11 \\
12 \\
13 \\
14 \\
15 \\
16 \\
17 \\
18 \\
19 \\
20 \\
21 \\
22 \\
23 \\
24 \\
25 \\
26 \\
27 \\
28 \\
29 \\
30 \\
31\end{array}$ & $\begin{array}{l}76.0 \\
77.0 \\
78.5 \\
77.0 \\
77.0 \\
77.0 \\
75.5 \\
75.0 \\
75.0 \\
74.5 \\
74.5 \\
74.5 \\
74.0 \\
74.0 \\
76.5 \\
76.0 \\
76.0 \\
76.0 \\
76.0 \\
75.0 \\
75.5 \\
76.0 \\
76.3 \\
76.3 \\
76.3 \\
76.3 \\
76.5 \\
78.0 \\
77.0 \\
72.3 \\
72.3\end{array}$ & $\begin{array}{l}85.0 \\
85.0 \\
84.0 \\
84.5 \\
84.5 \\
84.5 \\
85.0 \\
85.0 \\
85.0 \\
85.5 \\
85.5 \\
85.5 \\
86.0 \\
86.0 \\
86.0 \\
85.0 \\
85.0 \\
85.0 \\
85.0 \\
84.0 \\
85.0 \\
84.0 \\
87.0 \\
87.0 \\
87.0 \\
87.0 \\
90.0 \\
89.0 \\
86.0 \\
85.5 \\
85.5\end{array}$ & $\begin{array}{l}67.0 \\
69.0 \\
73.0 \\
69.5 \\
69.5 \\
69.5 \\
66.0 \\
65.0 \\
65.0 \\
63.5 \\
63.5 \\
63.5 \\
62.0 \\
62.0 \\
67.0 \\
67.0 \\
67.0 \\
67.0 \\
67.0 \\
66.0 \\
65.0 \\
68.0 \\
65.5 \\
65.5 \\
65.5 \\
65.5 \\
53.0 \\
67.0 \\
68.0 \\
64.0 \\
64.0\end{array}$ & $\begin{array}{l}1.03 \\
1.123 \\
0.38 \\
0.38 \\
0.38 \\
0.38 \\
0.90 \\
1.02 \\
0.95 \\
0.95 \\
0.95 \\
0.95 \\
0.95 \\
0.64 \\
1.21 \\
0.75 \\
1.06 \\
1.06 \\
1.06 \\
0.92 \\
0.74 \\
0.86 \\
0.77 \\
0.77 \\
0.77 \\
0.77 \\
0.77 \\
0.77 \\
1.18 \\
1.15 \\
0.90 \\
0.90 \\
0.90\end{array}$ & $\begin{array}{r}24.7 \\
29.5 \\
9.1 \\
9.1 \\
9.1 \\
9.1 \\
21.6 \\
24.5 \\
22.8 \\
22.8 \\
22.8 \\
22.8 \\
15.4 \\
29.0 \\
18.0 \\
25.4 \\
25.4 \\
25.4 \\
22.1 \\
17.7 \\
20.6 \\
18.5 \\
18.5 \\
18.5 \\
18.5 \\
18.5 \\
28.3 \\
27.6 \\
21.6 \\
21.6 \\
21.6\end{array}$ & $\begin{array}{l}0.10 \\
0.23 \\
0.04 \\
0.04 \\
0.04 \\
0.05 \\
0.02 \\
0.01 \\
0.04 \\
0.04 \\
0.04 \\
0.04 \\
0.01 \\
0.02 \\
0.24 \\
0.14 \\
0.15 \\
0.15 \\
0.01 \\
0.01 \\
0.04 \\
0.00 \\
0.00 \\
0.01 \\
0.01 \\
0.01 \\
0.03 \\
0.07 \\
0.29 \\
0.30 \\
0.30\end{array}$ & $\begin{array}{l}0.144 \\
0.336 \\
0.072 \\
0.072 \\
0.072 \\
0.072 \\
0.168 \\
0.144 \\
0.168 \\
0.168 \\
0.168 \\
0.168 \\
0.168 \\
0.168 \\
0.384 \\
0.048 \\
0.000 \\
0.000 \\
0.1444 \\
0.168 \\
0.168 \\
0.1444 \\
0.144 \\
0.144 \\
0.1444 \\
0.144 \\
0.144 \\
0.192 \\
0.144 \\
0.144 \\
0.144\end{array}$ \\
\hline $\begin{array}{l}\text { MEAN } \\
\text { DAILY } \\
\text { VALUE }\end{array}$ & 75.7 & 85.6 & 68.3 & 0.86 & 20.6 & 0.08 & 0.139 \\
\hline $\begin{array}{l}\text { STANDARD } \\
\text { DEVIATION }\end{array}$ & 1.39 & 1.32 & 3.22 & 0.234 & 5.62 & 0.094 & 0.075 \\
\hline $\begin{array}{l}\text { EXTREME } \\
\text { DAILY } \\
\text { VALUE }\end{array}$ & NA & 90.0 & 62.0 & NA & $\begin{array}{l}\text { MAX } \\
29.5 \\
\text { MIN } \\
9.1 \\
\end{array}$ & $\begin{array}{l}\text { MAX } \\
0.29 \\
\text { MIN } \\
0.00 \\
\end{array}$ & $\begin{array}{c}\text { MAX } \\
0.384 \\
\text { MIN } \\
0.000\end{array}$ \\
\hline $\begin{array}{l}\text { TOTAL } \\
\text { FOR THE } \\
\text { MONTH }\end{array}$ & NA & NA & NA & NA & 638.6 & 2.47 & 4.296 \\
\hline
\end{tabular}


Table 2. (Continued)

MANATÍ JANUARY 1984

\begin{tabular}{|c|c|c|c|c|c|c|c|}
\hline DAY & $\begin{array}{l}\text { MEAN } \\
\left({ }^{\circ} \mathrm{F}\right)\end{array}$ & $\begin{array}{c}\text { EMPERA } \\
\text { MAX } \\
\left({ }^{\circ} \mathrm{F}\right)\end{array}$ & $\begin{array}{l}\text { MIN } \\
\left({ }^{\circ} F\right)\end{array}$ & \multicolumn{2}{|c|}{$\begin{array}{c}\text { WIND } \\
\text { VELOCITY }\end{array}$} & $\begin{array}{c}\text { DAILY } \\
\text { RAINFALL } \\
\text { (IN/DAY) }\end{array}$ & $\begin{array}{c}\text { PAN } \\
\text { EVAPORATION } \\
\text { (IN/DAY) }\end{array}$ \\
\hline $\begin{array}{l}1 \\
2 \\
3 \\
4 \\
5 \\
6 \\
7 \\
8 \\
9 \\
10 \\
11 \\
12 \\
13 \\
14 \\
15 \\
16 \\
17 \\
18 \\
19 \\
20 \\
21 \\
22 \\
23 \\
24 \\
25 \\
26 \\
27 \\
28 \\
29 \\
30 \\
31\end{array}$ & $\begin{array}{l}72.3 \\
72.3 \\
67.5 \\
74.0 \\
74.5 \\
70.0 \\
70.0 \\
70.0 \\
65.5 \\
75.0 \\
74.0 \\
74.0 \\
74.0 \\
75.0 \\
75.0 \\
76.0 \\
76.3 \\
76.5 \\
75.0 \\
73.5 \\
74.0 \\
74.0 \\
74.5 \\
74.4 \\
74.4 \\
74.4 \\
74.4 \\
74.4 \\
74.4 \\
74.4 \\
74.4\end{array}$ & $\begin{array}{l}85.5 \\
85.5 \\
85.0 \\
84.0 \\
86.0 \\
87.5 \\
87.5 \\
87.5 \\
89.0 \\
83.0 \\
83.0 \\
83.0 \\
83.0 \\
85.0 \\
85.0 \\
87.0 \\
87.5 \\
88.0 \\
86.0 \\
84.0 \\
85.0 \\
85.0 \\
86.0 \\
86.0 \\
86.0 \\
86.0 \\
86.0 \\
86.0 \\
86.0 \\
86.0 \\
86.0\end{array}$ & $\begin{array}{l}64.0 \\
64.0 \\
60.0 \\
64.0 \\
63.0 \\
62.5 \\
62.5 \\
62.5 \\
62.0 \\
67.0 \\
65.0 \\
65.0 \\
65.0 \\
65.0 \\
65.0 \\
65.0 \\
65.0 \\
65.0 \\
64.0 \\
63.0 \\
63.0 \\
63.0 \\
63.0 \\
62.8 \\
62.8 \\
62.8 \\
62.8 \\
62.8 \\
62.8 \\
62.8 \\
62.8\end{array}$ & $\begin{array}{l}0.90 \\
0.90 \\
0.54 \\
0.56 \\
0.70 \\
0.70 \\
0.70 \\
0.70 \\
1.05 \\
1.24 \\
0.93 \\
0.93 \\
1.10 \\
1.10 \\
1.10 \\
1.10 \\
1.10 \\
1.33 \\
1.33 \\
1.27 \\
1.27 \\
1.27 \\
1.36 \\
1.36 \\
1.36 \\
1.36 \\
1.36 \\
1.36 \\
1.36 \\
1.36 \\
1.36\end{array}$ & $\begin{array}{l}21.6 \\
21.6 \\
12.9 \\
13.5 \\
16.7 \\
16.7 \\
16.7 \\
16.7 \\
25.2 \\
29.8 \\
22.3 \\
22.3 \\
26.4 \\
26.4 \\
26.4 \\
26.4 \\
26.4 \\
31.9 \\
31.9 \\
30.5 \\
30.5 \\
30.5 \\
32.6 \\
32.6 \\
32.6 \\
32.6 \\
32.6 \\
32.6 \\
32.6 \\
32.6 \\
32.6\end{array}$ & $\begin{array}{l}0.30 \\
0.30 \\
0.04 \\
0.01 \\
0.06 \\
0.06 \\
0.06 \\
0.07 \\
0.45 \\
0.03 \\
0.05 \\
0.05 \\
0.04 \\
0.04 \\
0.04 \\
0.02 \\
0.03 \\
0.07 \\
0.07 \\
0.04 \\
0.04 \\
0.04 \\
0.00 \\
0.00 \\
0.00 \\
0.00 \\
0.00 \\
0.00 \\
0.00 \\
0.00 \\
0.00\end{array}$ & $\begin{array}{l}0.144 \\
0.144 \\
0.144 \\
0.192 \\
0.144 \\
0.144 \\
0.144 \\
0.144 \\
0.168 \\
0.216 \\
0.168 \\
0.168 \\
0.168 \\
0.168 \\
0.168 \\
0.216 \\
0.216 \\
0.168 \\
0.168 \\
0.192 \\
0.192 \\
0.192 \\
0.000 \\
0.000 \\
0.000 \\
0.000 \\
0.000 \\
0.000 \\
0.000 \\
0.000 \\
0.000\end{array}$ \\
\hline $\begin{array}{l}\text { MEAN } \\
\text { DAILY } \\
\text { VALUE }\end{array}$ & 73.5 & 85.7 & 67.6 & 1.10 & 26.3 & 0.06 & 0.122 \\
\hline $\begin{array}{l}\text { STANDARD } \\
\text { DEVIATION }\end{array}$ & 2.42 & 1.49 & 4.26 & 0.267 & 6.48 & 0.100 & 0.080 \\
\hline $\begin{array}{l}\text { EXTREME } \\
\text { DAILY } \\
\text { VALUE }\end{array}$ & $\mathrm{NA}$ & 89.0 & 60.0 & $\mathrm{NA}$ & $\begin{array}{l}\text { MAX } \\
32.6 \\
\text { MIN } \\
12.9\end{array}$ & $\begin{array}{l}\text { MAX } \\
0.45 \\
\text { MIN } \\
0.00\end{array}$ & $\begin{array}{c}\text { MAX } \\
0.216 \\
\text { MIN } \\
0.144\end{array}$ \\
\hline $\begin{array}{l}\text { TOTAL } \\
\text { FOR THE } \\
\text { MONTH }\end{array}$ & NA & NA & NA & NA & 815.3 & 1.91 & 3.768 \\
\hline
\end{tabular}


Table 2. (Continued)

MANATI FEBRUARY 1984

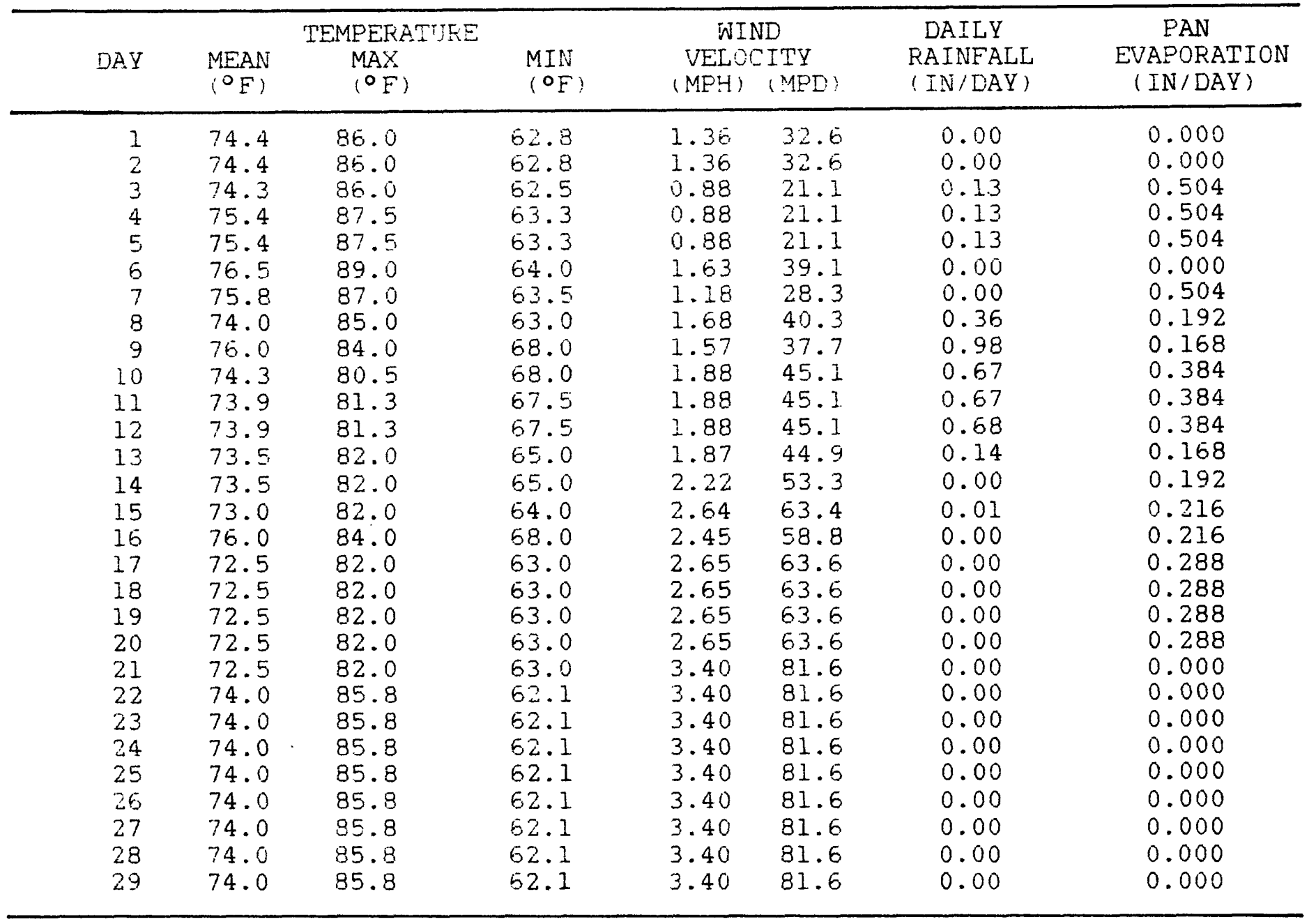

MEAN

DAILY

VALUE

$74.1 \quad 84.4$

63.8

$2.27 \quad 55.8$

0.13

0.320

STANDARD

DEVIATION

1.09

2.29

1.99

$0.886 \quad 21.2$

0.262

0.093

EXTREME

DAILY

NA $\quad 89.0$

62.1

MAX

NA

81.6

MAX

MIN

0.98

MIN

MAX

VALUE

21.1

0.00

MIN

TOTAL

FOR THE

NA NA

NA

NA 1618.2

3.91

9.287

MONTH 
Table 2. (Continued)

MANATI

MARCH 1984

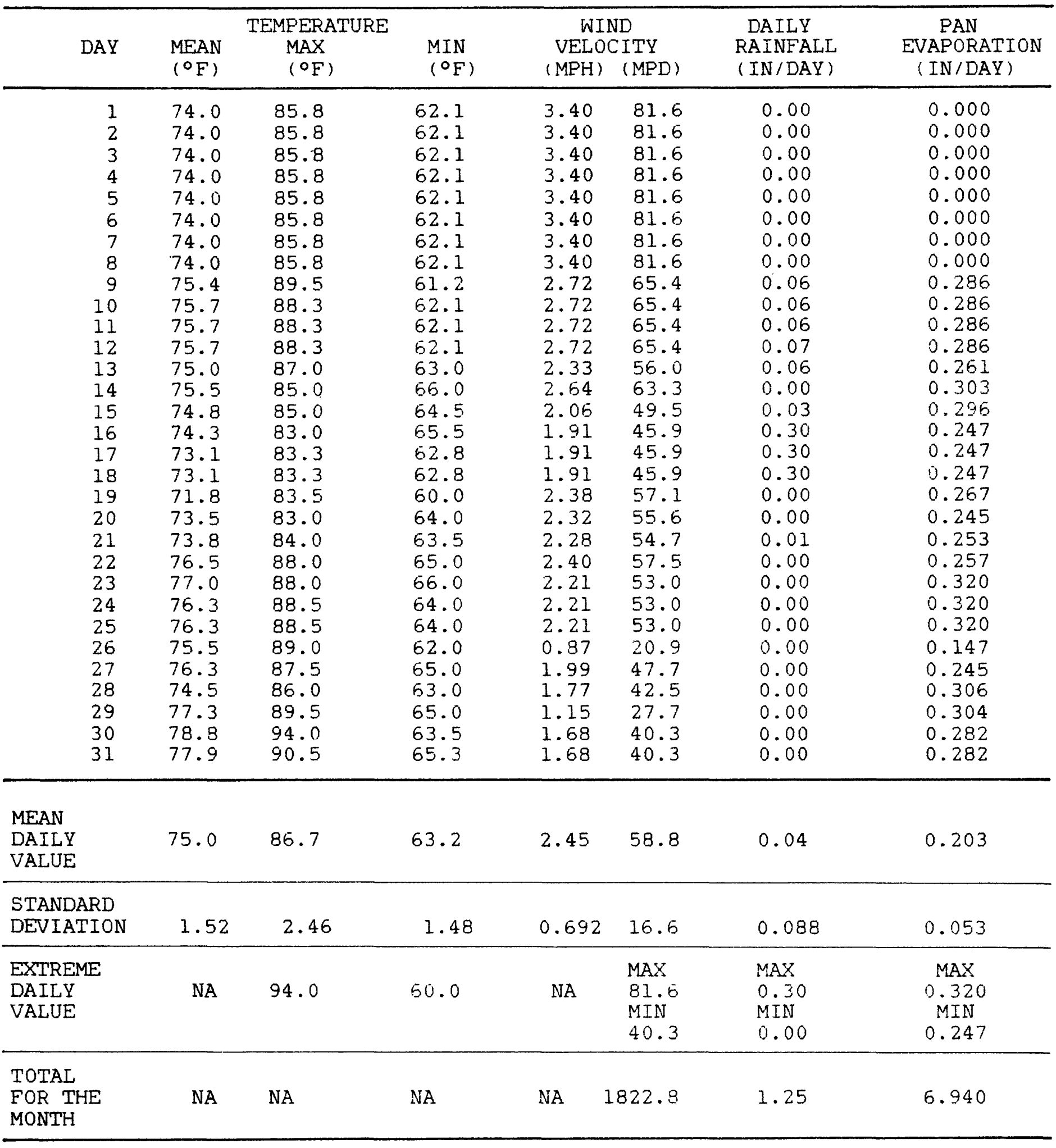


Table 2. (Continued)

MANATI APRIL 1984

\begin{tabular}{|c|c|c|c|c|c|c|c|}
\hline DAY & $\begin{array}{l}\text { MEAN } \\
(O F)\end{array}$ & $\begin{array}{l}\text { TEMPERATURE } \\
\text { MAX } \\
\left({ }^{\circ} \mathrm{F}\right)\end{array}$ & $\begin{array}{l}\text { MIN } \\
\left({ }^{\circ} \mathrm{F}\right)\end{array}$ & $\begin{array}{r}\text { WI } \\
V E L O \\
(M P H)\end{array}$ & $\begin{array}{l}\text { ND } \\
\text { OITY } \\
\text { (MPD) }\end{array}$ & $\begin{array}{l}\text { DAILY } \\
\text { FAINFALL } \\
\text { (IN/DAI) }\end{array}$ & $\begin{array}{c}\text { PAN } \\
\text { EVAPORATION } \\
\text { (IN/DAY) }\end{array}$ \\
\hline $\begin{array}{l}1 \\
2 \\
3 \\
4 \\
5 \\
6 \\
7 \\
8 \\
9 \\
10 \\
11 \\
12 \\
13 \\
14 \\
15 \\
16 \\
17 \\
18 \\
19 \\
20 \\
21 \\
22 \\
23 \\
24 \\
25 \\
26 \\
27 \\
28 \\
29 \\
30\end{array}$ & $\begin{array}{l}77.9 \\
77.0 \\
76.5 \\
75.0 \\
75.0 \\
76.5 \\
78.5 \\
81.8 \\
74.5 \\
74.0 \\
80.0 \\
76.5 \\
78.5 \\
77.0 \\
79.0 \\
75.5 \\
78.0 \\
75.5 \\
76.0 \\
76.0 \\
76.5 \\
76.3 \\
76.0 \\
75.0 \\
82.5 \\
77.0 \\
77.5 \\
73.0 \\
73.0 \\
71.5\end{array}$ & $\begin{array}{r}90.5 \\
87.0 \\
84.0 \\
82.0 \\
86.0 \\
91.0 \\
93.0 \\
100.5 \\
86.0 \\
87.0 \\
94.0 \\
88.0 \\
89.0 \\
87.0 \\
93.0 \\
87.0 \\
91.0 \\
87.0 \\
86.0 \\
85.0 \\
85.0 \\
85.0 \\
85.0 \\
88.0 \\
90.0 \\
88.0 \\
87.0 \\
85.0 \\
82.0 \\
81.0\end{array}$ & $\begin{array}{l}65.3 \\
67.0 \\
69.0 \\
68.0 \\
54.0 \\
62.0 \\
64.0 \\
63.0 \\
65.0 \\
61.0 \\
66.0 \\
65.0 \\
68.0 \\
67.0 \\
65.0 \\
64.0 \\
65.0 \\
64.0 \\
66.0 \\
67.0 \\
68.0 \\
67.5 \\
67.0 \\
62.0 \\
75.0 \\
66.0 \\
68.0 \\
61.0 \\
64.0 \\
62.0\end{array}$ & $\begin{array}{l}1.68 \\
1.55 \\
1.80 \\
2.42 \\
1.33 \\
1.57 \\
1.57 \\
1.50 \\
2.63 \\
1.04 \\
1.30 \\
1.39 \\
2.61 \\
2.52 \\
2.52 \\
0.68 \\
1.90 \\
2.24 \\
1.57 \\
1.57 \\
1.57 \\
1.57 \\
1.24 \\
1.24 \\
0.91 \\
1.06 \\
2.40 \\
1.30 \\
1.30 \\
0.85\end{array}$ & $\begin{array}{l}40.3 \\
37.2 \\
43.2 \\
59.3 \\
31.9 \\
37.7 \\
37.7 \\
37.7 \\
63.1 \\
25.0 \\
31.2 \\
33.4 \\
62.6 \\
60.5 \\
60.5 \\
16.3 \\
45.6 \\
53.8 \\
37.7 \\
37.7 \\
37.7 \\
37.7 \\
29.8 \\
29.8 \\
21.8 \\
25.4 \\
57.6 \\
31.2 \\
31.2 \\
20.4\end{array}$ & $\begin{array}{l}0.00 \\
0.07 \\
0.02 \\
0.00 \\
0.00 \\
0.00 \\
0.00 \\
0.00 \\
0.00 \\
0.00 \\
0.00 \\
0.00 \\
0.40 \\
0.40 \\
0.40 \\
0.00 \\
0.00 \\
0.00 \\
0.00 \\
0.00 \\
0.00 \\
0.00 \\
0.00 \\
0.00 \\
0.02 \\
0.00 \\
0.02 \\
0.02 \\
0.02 \\
0.00\end{array}$ & $\begin{array}{l}0.282 \\
0.267 \\
0.170 \\
0.287 \\
0.287 \\
0.286 \\
0.286 \\
0.286 \\
0.326 \\
0.350 \\
0.304 \\
0.300 \\
0.323 \\
0.323 \\
0.323 \\
0.224 \\
0.227 \\
0.266 \\
0.316 \\
0.316 \\
0.316 \\
0.316 \\
0.227 \\
0.197 \\
0.289 \\
0.253 \\
0.283 \\
0.283 \\
0.283 \\
0.013\end{array}$ \\
\hline $\begin{array}{l}\text { MEAN } \\
\text { DAILY } \\
\text { VALUE }\end{array}$ & 76.6 & 87.7 & 65.5 & 1.63 & 39.2 & 0.05 & 0.274 \\
\hline $\begin{array}{l}\text { STANDARD } \\
\text { DEVIATION }\end{array}$ & 2.35 & 3.95 & 2.81 & 0.54 & 13.0 & 0.119 & 0.063 \\
\hline $\begin{array}{l}\text { EXTREME } \\
\text { DAILY } \\
\text { VALUE }\end{array}$ & NA & 100.5 & 61.0 & $\mathrm{NA}$ & $\begin{array}{l}\text { MAX } \\
62.6 \\
\text { MIN } \\
16.3\end{array}$ & $\begin{array}{l}\text { MAX } \\
0.40 \\
\text { MIN } \\
0.00\end{array}$ & $\begin{array}{c}\text { MAX } \\
0.350 \\
\text { MIN } \\
0.013\end{array}$ \\
\hline $\begin{array}{l}\text { TOTAL } \\
\text { FOR THE } \\
\text { MONTH }\end{array}$ & NA & NA & $\mathrm{NA}$ & NA & 1176.0 & 1.35 & 8.209 \\
\hline
\end{tabular}


Table 2. (Continued)

MANATÍ MAY 1984

\begin{tabular}{|c|c|c|c|c|c|c|c|}
\hline DAY & $\begin{array}{l}\text { MEAN } \\
\left({ }^{\circ} \mathrm{F}\right)\end{array}$ & $\begin{array}{l}\text { EMPERAT } \\
\text { MAX } \\
\left({ }^{\circ} \mathrm{F}\right)\end{array}$ & $\begin{array}{l}\text { MIN } \\
(O F)\end{array}$ & \multicolumn{2}{|c|}{$\begin{array}{c}\text { WIND } \\
\text { VELOCITY }\end{array}$} & $\begin{array}{l}\text { DAILY } \\
\text { RAINFALL } \\
\text { (IN/DAY) }\end{array}$ & $\begin{array}{c}\text { PAN } \\
\text { EVAPORATION } \\
\text { (IN/DAY) }\end{array}$ \\
\hline $\begin{array}{l}1 \\
2 \\
3 \\
4 \\
5 \\
6 \\
7 \\
8 \\
9 \\
10 \\
11 \\
12 \\
13 \\
14 \\
15 \\
16 \\
17 \\
18 \\
19 \\
20 \\
21 \\
22 \\
23 \\
24 \\
25 \\
26 \\
27 \\
28 \\
29 \\
30 \\
31\end{array}$ & $\begin{array}{l}78.5 \\
75.0 \\
77.0 \\
77.5 \\
77.0 \\
77.5 \\
79.0 \\
76.5 \\
76.5 \\
77.0 \\
79.0 \\
78.0 \\
76.5 \\
75.0 \\
76.0 \\
76.0 \\
76.3 \\
77.0 \\
75.5 \\
78.0 \\
78.3 \\
78.8 \\
76.8 \\
76.0 \\
75.0 \\
76.0 \\
77.0 \\
78.0 \\
77.0 \\
77.5 \\
79.3\end{array}$ & $\begin{array}{l}81.0 \\
81.5 \\
84.0 \\
85.0 \\
85.0 \\
86.0 \\
88.0 \\
85.0 \\
84.0 \\
86.0 \\
89.0 \\
87.0 \\
85.0 \\
82.0 \\
83.0 \\
83.0 \\
85.5 \\
85.0 \\
83.0 \\
85.0 \\
86.5 \\
85.5 \\
84.0 \\
84.0 \\
82.0 \\
84.0 \\
85.0 \\
86.0 \\
85.0 \\
85.0 \\
87.0\end{array}$ & $\begin{array}{l}66.0 \\
68.5 \\
70.0 \\
70.0 \\
69.0 \\
69.0 \\
70.0 \\
68.0 \\
69.0 \\
68.0 \\
69.0 \\
69.0 \\
68.0 \\
68.0 \\
69.0 \\
69.0 \\
67.0 \\
69.0 \\
68.0 \\
71.0 \\
70.0 \\
70.0 \\
69.5 \\
68.0 \\
68.0 \\
68.0 \\
69.0 \\
70.0 \\
69.0 \\
70.0 \\
70.5\end{array}$ & $\begin{array}{l}0.98 \\
2.04 \\
2.14 \\
2.24 \\
1.88 \\
2.45 \\
1.90 \\
2.13 \\
2.07 \\
1.44 \\
2.10 \\
1.31 \\
1.31 \\
1.55 \\
1.40 \\
1.35 \\
0.77 \\
0.55 \\
1.23 \\
1.66 \\
1.12 \\
1.78 \\
1.07 \\
0.79 \\
0.55 \\
0.41 \\
0.98 \\
1.21 \\
0.95 \\
0.49 \\
0.37\end{array}$ & $\begin{array}{r}23.6 \\
49.0 \\
51.4 \\
53.8 \\
45.1 \\
58.8 \\
45.6 \\
51.1 \\
49.6 \\
34.6 \\
50.4 \\
31.4 \\
31.4 \\
37.2 \\
33.6 \\
32.4 \\
18.5 \\
13.2 \\
29.5 \\
39.8 \\
26.9 \\
42.7 \\
25.7 \\
19.0 \\
13.2 \\
9.8 \\
23.5 \\
29.0 \\
22.8 \\
11.8 \\
8.9\end{array}$ & $\begin{array}{l}0.01 \\
0.04 \\
0.00 \\
0.00 \\
0.00 \\
0.00 \\
0.00 \\
0.05 \\
0.00 \\
0.00 \\
0.03 \\
0.03 \\
0.04 \\
0.00 \\
0.06 \\
1.93 \\
0.09 \\
0.18 \\
0.19 \\
0.03 \\
0.05 \\
0.16 \\
0.46 \\
0.00 \\
1.02 \\
0.00 \\
0.00 \\
0.00 \\
0.06 \\
0.00 \\
0.01\end{array}$ & $\begin{array}{l}0.144 \\
0.336 \\
0.408 \\
0.312 \\
0.312 \\
0.312 \\
0.312 \\
0.192 \\
0.432 \\
0.312 \\
0.288 \\
0.288 \\
0.288 \\
0.288 \\
0.336 \\
0.528 \\
0.306 \\
0.552 \\
0.552 \\
0.552 \\
0.192 \\
0.312 \\
0.216 \\
0.240 \\
0.317 \\
0.317 \\
0.317 \\
0.317 \\
0.317 \\
0.192 \\
0.264\end{array}$ \\
\hline $\begin{array}{l}\text { MEAN } \\
\text { DAILY } \\
\text { VALUE }\end{array}$ & 77.0 & 84.7 & 68.9 & 1.36 & 32.7 & 0.14 & 0.324 \\
\hline $\begin{array}{l}\text { STANDARD } \\
\text { DEVIATION }\end{array}$ & 1.19 & 1.80 & 1.06 & 0.591 & 14.2 & 0.379 & 0.103 \\
\hline $\begin{array}{l}\text { EXTREME } \\
\text { DAILY } \\
\text { VALUE }\end{array}$ & NA & 89.0 & 66.0 & $\mathrm{NA}$ & $\begin{array}{l}\text { MAX } \\
58.8 \\
\text { MIN } \\
8.9\end{array}$ & $\begin{array}{l}\text { MAX } \\
1.93 \\
\text { MIN } \\
0.00\end{array}$ & $\begin{array}{c}\text { MAX } \\
0.552 \\
\text { MIN } \\
0.144\end{array}$ \\
\hline $\begin{array}{l}\text { TOTAL } \\
\text { FOR THE } \\
\text { MONTH }\end{array}$ & NA & NA & NA & NA & 1013.7 & 4.33 & 10.051 \\
\hline
\end{tabular}


Table 2. (Continued)

MANATI JUNE 1984

\begin{tabular}{|c|c|c|c|c|c|c|c|}
\hline EAY & $\begin{array}{l}\text { MEAN } \\
(O F)\end{array}$ & $\begin{array}{l}\text { TEMPERATURE } \\
\text { MAX } \\
(O \mathrm{~F})\end{array}$ & $\begin{array}{l}M I N \\
(O F)\end{array}$ & $\begin{array}{r}\text { WII } \\
V E L O U \\
(M P H)\end{array}$ & $\begin{array}{l}\text { VD } \\
\text { ITY } \\
\text { (MPD) }\end{array}$ & $\begin{array}{l}\text { DAILY } \\
\text { RAINFALL } \\
\text { (IN/DAY) }\end{array}$ & $\begin{array}{c}\text { PAN } \\
\text { EVAPORATION } \\
\text { (IN/DAY) }\end{array}$ \\
\hline $\begin{array}{l}1 \\
2 \\
3 \\
4 \\
5 \\
6 \\
7 \\
8 \\
9 \\
10 \\
11 \\
12 \\
13 \\
14 \\
15 \\
16 \\
17 \\
18 \\
19 \\
20 \\
21 \\
22 \\
23 \\
24 \\
25 \\
26 \\
27 \\
28 \\
29 \\
30\end{array}$ & $\begin{array}{l}79.4 \\
79.4 \\
79.4 \\
79.4 \\
79.5 \\
79.3 \\
79.0 \\
78.0 \\
78.0 \\
78.0 \\
80.0 \\
77.8 \\
76.5 \\
79.3 \\
79.7 \\
79.7 \\
79.7 \\
80.0 \\
76.5 \\
76.5 \\
78.0 \\
77.5 \\
78.3 \\
78.3 \\
79.0 \\
79.0 \\
79.0 \\
78.5 \\
78.5 \\
79.0\end{array}$ & $\begin{array}{l}87.0 \\
87.0 \\
87.0 \\
87.0 \\
87.0 \\
87.0 \\
87.0 \\
86.0 \\
86.0 \\
85.0 \\
88.0 \\
85.5 \\
81.5 \\
89.0 \\
89.0 \\
89.0 \\
89.0 \\
89.0 \\
86.0 \\
85.0 \\
87.0 \\
86.0 \\
87.5 \\
87.5 \\
89.0 \\
88.5 \\
88.0 \\
87.0 \\
87.0 \\
89.0\end{array}$ & $\begin{array}{l}71.3 \\
71.3 \\
71.3 \\
71.3 \\
72.0 \\
71.5 \\
71.0 \\
70.0 \\
70.0 \\
71.0 \\
72.0 \\
70.0 \\
71.5 \\
69.5 \\
70.3 \\
70.3 \\
70.3 \\
71.0 \\
69.0 \\
68.0 \\
69.0 \\
69.0 \\
59.0 \\
69.0 \\
69.0 \\
69.5 \\
70.0 \\
70.0 \\
68.0 \\
69.0\end{array}$ & $\begin{array}{l}0.37 \\
0.37 \\
0.37 \\
0.37 \\
0.13 \\
0.13 \\
0.10 \\
0.23 \\
0.23 \\
0.56 \\
0.28 \\
0.96 \\
0.56 \\
0.56 \\
0.56 \\
0.56 \\
0.56 \\
0.56 \\
0.56 \\
0.56 \\
0.56 \\
0.56 \\
0.56 \\
0.56 \\
0.56 \\
0.56 \\
0.56 \\
0.56 \\
0.56 \\
0.56\end{array}$ & $\begin{array}{r}8.9 \\
8.9 \\
8.9 \\
8.9 \\
3.2 \\
3.2 \\
2.3 \\
5.7 \\
5.7 \\
13.5 \\
5.8 \\
22.9 \\
13.4 \\
13.4 \\
13.4 \\
13.4 \\
13.4 \\
13.4 \\
13.4 \\
13.4 \\
13.4 \\
13.4 \\
13.4 \\
13.4 \\
13.4 \\
13.4 \\
13.4 \\
13.4 \\
13.4 \\
13.4\end{array}$ & $\begin{array}{l}0.01 \\
0.00 \\
0.00 \\
0.00 \\
0.95 \\
0.95 \\
0.95 \\
0.27 \\
0.01 \\
0.02 \\
0.01 \\
0.95 \\
0.05 \\
0.05 \\
0.05 \\
0.05 \\
0.05 \\
0.21 \\
0.53 \\
0.00 \\
0.00 \\
0.00 \\
0.00 \\
0.00 \\
0.00 \\
0.00 \\
0.00 \\
0.00 \\
0.09 \\
0.10\end{array}$ & $\begin{array}{l}0.264 \\
0.264 \\
0.264 \\
0.264 \\
0.293 \\
0.293 \\
0.233 \\
0.152 \\
0.152 \\
0.152 \\
0.172 \\
0.332 \\
0.349 \\
0.154 \\
0.154 \\
0.154 \\
0.154 \\
0.125 \\
0.214 \\
0.205 \\
0.193 \\
0.281 \\
0.281 \\
0.281 \\
0.279 \\
0.279 \\
0.280 \\
0.207 \\
0.254 \\
0.254\end{array}$ \\
\hline $\begin{array}{l}\text { MEAN } \\
\text { DAILY } \\
\text { VALUE }\end{array}$ & 78.7 & 87.1 & 70.1 & 0.47 & 11.3 & 0.18 & 0.231 \\
\hline $\begin{array}{l}\text { STANDARD } \\
\text { DEVIATION }\end{array}$ & 0.988 & 1.60 & 1.12 & 0.178 & 4.23 & 0.322 & 0.061 \\
\hline $\begin{array}{l}\text { EXTREME } \\
\text { DAILY } \\
\text { VALUE }\end{array}$ & NA & 89.0 & 68.0 & NA & $\begin{array}{l}\operatorname{MAX} \\
22.9 \\
\operatorname{MIN} \\
2.3\end{array}$ & $\begin{array}{l}\text { MAX } \\
0.95 \\
\text { MIN } \\
0.00\end{array}$ & $\begin{array}{c}\text { MAX } \\
0.349 \\
\text { MIN } \\
0.125\end{array}$ \\
\hline $\begin{array}{l}\text { TOTAL } \\
\text { FOR THE } \\
\text { MONTH }\end{array}$ & NA & NA & NA & NA & 339.0 & 5.31 & 6.930 \\
\hline
\end{tabular}


Table 2. (Continued)

MANATI JULY 1984

\begin{tabular}{|c|c|c|c|c|c|c|c|}
\hline DAY & $\begin{array}{l}\text { MEAN } \\
\left({ }^{\circ} \mathrm{F}\right)\end{array}$ & $\begin{array}{c}\text { MEMPERA } \\
\text { MAX } \\
\left({ }^{\circ} F\right)\end{array}$ & $\begin{array}{l}\text { MIN } \\
\left({ }^{\circ} \mathrm{F}\right)\end{array}$ & \multicolumn{2}{|c|}{$\begin{array}{c}\text { WIND } \\
\text { VELOCITY }\end{array}$} & $\begin{array}{l}\text { DAILY } \\
\text { RAINFALL } \\
\text { (IN/DAY) }\end{array}$ & $\begin{array}{c}\text { PAN } \\
\text { EVAPORATION } \\
\text { (IN/DAY) }\end{array}$ \\
\hline $\begin{array}{l}1 \\
2 \\
3 \\
4 \\
5 \\
6 \\
7 \\
8 \\
9 \\
10 \\
11 \\
12 \\
13 \\
14 \\
15 \\
16 \\
17 \\
18 \\
19 \\
20 \\
21 \\
22 \\
23 \\
24 \\
25 \\
26 \\
27 \\
28 \\
29 \\
30 \\
31\end{array}$ & $\begin{array}{l}79.0 \\
79.5 \\
78.8 \\
78.8 \\
78.0 \\
78.5 \\
78.8 \\
78.8 \\
79.0 \\
77.0 \\
77.8 \\
78.5 \\
80.0 \\
79.8 \\
79.8 \\
79.5 \\
79.0 \\
78.5 \\
78.0 \\
77.5 \\
78.3 \\
78.3 \\
79.0 \\
80.0 \\
81.3 \\
82.5 \\
82.8 \\
81.8 \\
81.8 \\
80.8 \\
81.5\end{array}$ & $\begin{array}{l}89.0 \\
89.0 \\
87.5 \\
87.5 \\
86.0 \\
87.0 \\
87.5 \\
87.5 \\
88.0 \\
85.0 \\
86.0 \\
87.0 \\
88.0 \\
88.5 \\
88.5 \\
89.0 \\
88.5 \\
88.0 \\
87.5 \\
87.0 \\
87.5 \\
87.5 \\
88.0 \\
88.0 \\
87.5 \\
89.0 \\
93.0 \\
92.5 \\
92.5 \\
92.0 \\
92.0\end{array}$ & $\begin{array}{l}69.5 \\
70.0 \\
70.0 \\
70.0 \\
70.0 \\
71.0 \\
70.5 \\
70.5 \\
70.0 \\
69.0 \\
69.5 \\
70.0 \\
72.0 \\
71.0 \\
71.0 \\
70.0 \\
69.5 \\
69.0 \\
68.5 \\
68.0 \\
69.0 \\
69.0 \\
70.0 \\
72.0 \\
75.0 \\
76.0 \\
72.5 \\
71.0 \\
71.0 \\
69.5 \\
71.0\end{array}$ & $\begin{array}{l}0.56 \\
0.56 \\
0.56 \\
0.56 \\
0.56 \\
0.56 \\
0.56 \\
0.56 \\
0.56 \\
0.56 \\
0.56 \\
0.56 \\
0.56 \\
0.56 \\
0.56 \\
0.56 \\
0.56 \\
0.56 \\
0.56 \\
0.56 \\
0.56 \\
0.56 \\
1.75 \\
2.62 \\
2.59 \\
2.86 \\
1.60 \\
1.60 \\
1.60 \\
2.51 \\
1.87\end{array}$ & $\begin{array}{l}13.4 \\
13.4 \\
13.4 \\
13.4 \\
13.4 \\
13.4 \\
13.4 \\
13.4 \\
13.4 \\
13.4 \\
13.4 \\
13.4 \\
13.4 \\
13.4 \\
13.4 \\
13.4 \\
13.4 \\
13.4 \\
13.4 \\
13.4 \\
13.4 \\
13.4 \\
42.0 \\
62.8 \\
62.1 \\
68.7 \\
38.3 \\
38.3 \\
38.3 \\
60.3 \\
44.8\end{array}$ & $\begin{array}{l}0.10 \\
0.20 \\
0.20 \\
0.19 \\
0.00 \\
0.03 \\
0.02 \\
0.02 \\
0.00 \\
0.10 \\
0.09 \\
0.00 \\
0.17 \\
0.17 \\
0.16 \\
0.00 \\
0.00 \\
0.01 \\
0.00 \\
0.15 \\
0.15 \\
0.14 \\
0.12 \\
0.01 \\
0.04 \\
0.00 \\
0.08 \\
0.07 \\
0.07 \\
0.00 \\
0.00\end{array}$ & $\begin{array}{l}0.254 \\
0.193 \\
0.193 \\
0.193 \\
0.175 \\
0.263 \\
0.263 \\
0.263 \\
0.301 \\
0.171 \\
0.171 \\
0.280 \\
0.223 \\
0.223 \\
0.223 \\
0.272 \\
0.272 \\
0.206 \\
0.206 \\
0.150 \\
0.150 \\
0.150 \\
0.150 \\
0.095 \\
0.340 \\
0.418 \\
0.213 \\
0.213 \\
0.213 \\
0.318 \\
0.286\end{array}$ \\
\hline $\begin{array}{l}\text { MEAN } \\
\text { DAILY } \\
\text { VALUE }\end{array}$ & 77.2 & 88.4 & 70.5 & 1.01 & 24.2 & 0.07 & 0.227 \\
\hline $\begin{array}{l}\text { STANDARD } \\
\text { DEVIATION }\end{array}$ & 2.68 & 1.95 & 11.2 & 0.752 & 18.1 & 0.071 & 0.066 \\
\hline $\begin{array}{l}\text { EXTREME } \\
\text { DAILY } \\
\text { VALUE }\end{array}$ & NA & 93.0 & 68.0 & NA & $\begin{array}{l}\text { MAX } \\
68.7 \\
\text { MIN } \\
13.4\end{array}$ & $\begin{array}{l}\text { MAX } \\
0.20 \\
\text { MIN } \\
0.00\end{array}$ & $\begin{array}{c}\text { MAX } \\
0.418 \\
\text { MIN } \\
0.095\end{array}$ \\
\hline $\begin{array}{l}\text { TOTAL } \\
\text { FOR THE } \\
\text { MONTH }\end{array}$ & NA & NA & NA & NA & 750.2 & 2.29 & 7.041 \\
\hline
\end{tabular}


Table 2. (Continued)

MANATÍ AUGUST 1984

\begin{tabular}{|c|c|c|c|c|c|c|c|}
\hline$D A Y$ & $\begin{array}{l}\text { MEAN } \\
(\circ F)\end{array}$ & $\begin{array}{c}\text { TEMPEFATURE } \\
\text { MAX } \\
\left({ }^{\circ} \mathrm{F}\right)\end{array}$ & $\begin{array}{l}\text { MIN } \\
\text { OOF }\end{array}$ & $\begin{array}{r}\text { NI } \\
\text { VELO } \\
(M P H)\end{array}$ & $\begin{array}{l}\text { JD } \\
\text { ITY } \\
\text { (MPD) }\end{array}$ & $\begin{array}{l}\text { DAILY } \\
\text { RAINFALL } \\
\text { (IN/DAY) }\end{array}$ & $\begin{array}{c}\text { PAN } \\
\text { EVAPORATION } \\
(\text { IN / DAY })\end{array}$ \\
\hline $\begin{array}{l}1 \\
2 \\
3 \\
4 \\
5 \\
6 \\
7 \\
8 \\
9 \\
10 \\
11 \\
12 \\
13 \\
14 \\
15 \\
16 \\
17 \\
18 \\
19 \\
20 \\
21 \\
22 \\
23 \\
24 \\
25 \\
26 \\
27 \\
28 \\
29 \\
30 \\
31\end{array}$ & $\begin{array}{l}80.5 \\
80.0 \\
79.5 \\
79.8 \\
79.8 \\
79.0 \\
78.5 \\
79.0 \\
83.8 \\
81.8 \\
81.2 \\
81.2 \\
80.5 \\
80.0 \\
82.8 \\
81.0 \\
80.0 \\
79.3 \\
79.3 \\
78.5 \\
82.3 \\
81.4 \\
80.5 \\
80.8 \\
80.7 \\
80.7 \\
80.6 \\
80.5 \\
81.8 \\
80.8 \\
79.0\end{array}$ & $\begin{array}{l}90.0 \\
88.0 \\
88.0 \\
88.5 \\
88.5 \\
89.0 \\
89.0 \\
90.0 \\
93.0 \\
93.5 \\
92.5 \\
92.5 \\
91.5 \\
91.0 \\
93.5 \\
91.0 \\
90.0 \\
90.5 \\
90.5 \\
91.0 \\
93.0 \\
92.0 \\
91.0 \\
90.0 \\
90.5 \\
90.5 \\
90.5 \\
91.0 \\
93.5 \\
90.0 \\
89.0\end{array}$ & $\begin{array}{l}71.0 \\
72.0 \\
71.0 \\
70.0 \\
70.0 \\
69.0 \\
68.0 \\
68.0 \\
74.5 \\
70.0 \\
59.8 \\
69.8 \\
69.5 \\
69.0 \\
72.0 \\
71.0 \\
70.0 \\
68.0 \\
68.0 \\
66.0 \\
71.5 \\
70.8 \\
70.0 \\
71.5 \\
70.8 \\
70.8 \\
70.8 \\
70.0 \\
70.0 \\
71.5 \\
69.0\end{array}$ & $\begin{array}{l}1.62 \\
1.68 \\
1.07 \\
1.07 \\
1.07 \\
1.24 \\
1.15 \\
1.51 \\
1.42 \\
1.42 \\
1.42 \\
1.42 \\
1.46 \\
1.05 \\
0.61 \\
0.62 \\
1.12 \\
1.12 \\
1.12 \\
1.68 \\
0.95 \\
0.95 \\
1.49 \\
1.61 \\
1.61 \\
1.61 \\
1.61 \\
1.84 \\
1.58 \\
1.61 \\
1.23\end{array}$ & $\begin{array}{l}38.8 \\
40.4 \\
25.8 \\
25.8 \\
25.8 \\
29.8 \\
27.6 \\
36.1 \\
34.1 \\
34.0 \\
34.0 \\
34.0 \\
35.0 \\
25.3 \\
14.6 \\
14.9 \\
26.9 \\
26.9 \\
26.9 \\
40.4 \\
22.9 \\
22.9 \\
35.8 \\
38.6 \\
38.6 \\
38.6 \\
38.6 \\
44.2 \\
38.0 \\
38.5 \\
29.6\end{array}$ & $\begin{array}{l}0.80 \\
0.00 \\
0.25 \\
0.24 \\
0.24 \\
0.00 \\
0.00 \\
0.00 \\
0.00 \\
0.01 \\
0.00 \\
0.00 \\
0.01 \\
0.17 \\
0.40 \\
0.68 \\
0.00 \\
0.00 \\
0.00 \\
0.00 \\
0.26 \\
0.26 \\
0.00 \\
0.09 \\
0.09 \\
0.09 \\
0.09 \\
0.00 \\
0.01 \\
0.02 \\
0.06\end{array}$ & $\begin{array}{l}0.222 \\
0.206 \\
0.236 \\
0.236 \\
0.236 \\
0.270 \\
0.271 \\
0.273 \\
0.282 \\
0.251 \\
0.251 \\
0.251 \\
0.241 \\
0.183 \\
0.570 \\
0.167 \\
0.229 \\
0.229 \\
0.229 \\
0.301 \\
0.196 \\
0.196 \\
0.269 \\
0.171 \\
0.171 \\
0.171 \\
0.171 \\
0.282 \\
0.241 \\
0.295 \\
0.203\end{array}$ \\
\hline $\begin{array}{l}\text { MEAN } \\
\text { DAILY } \\
\text { VALUE }\end{array}$ & 80.5 & 90.7 & 70.1 & 1.37 & 31.7 & 0.12 & 0.242 \\
\hline $\begin{array}{l}\text { STANDARD } \\
\text { DEVIATION }\end{array}$ & 1.21 & 1.60 & 1.56 & 0.312 & 7.34 & 0.195 & 0.071 \\
\hline $\begin{array}{l}\text { EXTREME } \\
\text { DAILY } \\
\text { VALUE }\end{array}$ & $\mathrm{NA}$ & 93.5 & 66.0 & NA & $\begin{array}{l}\text { MAX } \\
44.4 \\
\text { MIN } \\
22.9\end{array}$ & $\begin{array}{l}\text { MAX } \\
0.80 \\
\text { MIN } \\
0.00\end{array}$ & $\begin{array}{c}\text { MAX } \\
0.570 \\
\text { MIN } \\
0.171\end{array}$ \\
\hline $\begin{array}{l}\text { TOTAL } \\
\text { FOR THE } \\
\text { MONTH }\end{array}$ & NA & NA & $\mathrm{NA}$ & NA & 982.7 & 3.71 & 7.502 \\
\hline
\end{tabular}


Table 2. (Continued)

MANATI SEPTEMBER 1984

\begin{tabular}{|c|c|c|c|c|c|c|c|}
\hline DAY & $\begin{array}{l}\text { MEAN } \\
(O F)\end{array}$ & $\begin{array}{c}\text { EMPERA } \\
\text { MAX } \\
\left({ }^{\circ} \mathrm{F}\right)\end{array}$ & $\begin{array}{l}\text { MIN } \\
(\circ \mathrm{F})\end{array}$ & $\begin{array}{r}\text { WII } \\
\text { VELO } \\
\text { (MPH) }\end{array}$ & $\begin{array}{l}\text { ND } \\
\text { CITY } \\
\text { (MPD) }\end{array}$ & $\begin{array}{c}\text { DAILY } \\
\text { RAINFALL } \\
(\text { IN/DAY })\end{array}$ & $\begin{array}{c}\text { PAN } \\
\text { EVAPORATION } \\
\text { (IN/DAY) }\end{array}$ \\
\hline $\begin{array}{r}1 \\
2 \\
3 \\
4 \\
5 \\
6 \\
7 \\
8 \\
9 \\
10 \\
11 \\
12 \\
13 \\
14 \\
15 \\
16 \\
17 \\
18 \\
19 \\
20 \\
21 \\
22 \\
23 \\
24 \\
25 \\
26 \\
27 \\
28 \\
29 \\
30\end{array}$ & $\begin{array}{l}78.8 \\
78.8 \\
78.8 \\
78.5 \\
79.8 \\
79.8 \\
81.0 \\
80.3 \\
80.3 \\
79.5 \\
78.5 \\
79.3 \\
80.0 \\
79.5 \\
79.8 \\
79.8 \\
80.0 \\
79.3 \\
78.5 \\
78.5 \\
80.3 \\
79.8 \\
79.8 \\
79.3 \\
80.3 \\
79.6 \\
79.6 \\
78.8 \\
79.3 \\
79.3\end{array}$ & $\begin{array}{l}88.5 \\
88.5 \\
88.5 \\
88.0 \\
90.0 \\
90.0 \\
92.0 \\
91.0 \\
91.0 \\
90.0 \\
90.0 \\
90.0 \\
90.0 \\
88.0 \\
89.0 \\
89.0 \\
90.0 \\
89.0 \\
88.0 \\
87.0 \\
89.5 \\
89.5 \\
89.5 \\
89.5 \\
91.5 \\
90.5 \\
90.5 \\
89.5 \\
90.0 \\
90.0\end{array}$ & $\begin{array}{l}69.0 \\
69.0 \\
69.0 \\
69.0 \\
69.5 \\
69.5 \\
70.0 \\
69.5 \\
69.5 \\
69.0 \\
67.0 \\
68.5 \\
70.0 \\
71.0 \\
70.5 \\
70.5 \\
70.0 \\
69.5 \\
69.0 \\
70.0 \\
71.0 \\
68.0 \\
68.0 \\
65.0 \\
69.0 \\
68.5 \\
68.5 \\
68.0 \\
68.5 \\
68.5\end{array}$ & $\begin{array}{l}1.23 \\
1.23 \\
1.23 \\
1.39 \\
1.39 \\
1.39 \\
1.68 \\
1.68 \\
1.68 \\
1.40 \\
0.90 \\
0.90 \\
1.01 \\
0.98 \\
0.98 \\
0.98 \\
0.44 \\
0.44 \\
0.48 \\
0.42 \\
0.48 \\
0.48 \\
0.48 \\
0.49 \\
0.84 \\
0.84 \\
0.84 \\
0.43 \\
0.43 \\
0.43\end{array}$ & $\begin{array}{l}29.6 \\
29.6 \\
29.6 \\
33.6 \\
33.6 \\
33.6 \\
40.3 \\
40.3 \\
40.3 \\
33.6 \\
21.7 \\
21.7 \\
24.3 \\
23.6 \\
23.6 \\
23.6 \\
10.5 \\
10.5 \\
11.5 \\
10.0 \\
11.5 \\
11.5 \\
11.5 \\
11.6 \\
20.2 \\
20.2 \\
20.2 \\
10.4 \\
10.4 \\
10.4\end{array}$ & $\begin{array}{l}0.05 \\
0.05 \\
0.05 \\
0.13 \\
0.13 \\
0.12 \\
0.00 \\
0.00 \\
0.00 \\
0.12 \\
0.40 \\
0.39 \\
0.20 \\
0.21 \\
0.21 \\
0.20 \\
0.97 \\
0.96 \\
0.14 \\
0.00 \\
0.02 \\
0.02 \\
0.01 \\
0.00 \\
0.06 \\
0.05 \\
0.05 \\
0.33 \\
0.33 \\
0.33\end{array}$ & $\begin{array}{l}0.203 \\
0.203 \\
0.203 \\
0.202 \\
0.202 \\
0.202 \\
0.266 \\
0.266 \\
0.266 \\
0.173 \\
0.127 \\
0.127 \\
0.112 \\
0.179 \\
0.179 \\
0.179 \\
0.128 \\
0.128 \\
0.135 \\
0.112 \\
0.152 \\
0.152 \\
0.152 \\
0.184 \\
0.183 \\
0.183 \\
0.183 \\
0.292 \\
0.292 \\
0.292\end{array}$ \\
\hline $\begin{array}{l}\text { MEAN } \\
\text { DAILY } \\
\text { VALUE }\end{array}$ & 79.5 & 89.6 & 69.1 & 0.93 & 22.1 & 0.18 & 0.189 \\
\hline $\begin{array}{l}\text { STANDARD } \\
\text { DEVIATION }\end{array}$ & 0.633 & 1.09 & 1.18 & 0.422 & 10.1 & 0.242 & 0.053 \\
\hline $\begin{array}{l}\text { EXTREME } \\
\text { DAILY } \\
\text { VALUE }\end{array}$ & NA & 92.0 & 65.0 & NA & $\begin{array}{l}\text { MAX } \\
40.3 \\
\text { MIN } \\
10.4\end{array}$ & $\begin{array}{l}\text { MAX } \\
0.97 \\
\text { MIN } \\
0.00\end{array}$ & $\begin{array}{c}\text { MAX } \\
0.292 \\
\text { MIN } \\
0.127\end{array}$ \\
\hline $\begin{array}{l}\text { TOTAL } \\
\text { FOR THE } \\
\text { MONTH }\end{array}$ & NA & $\mathrm{NA}$ & $\mathrm{NA}$ & NA & 663.0 & 5.53 & 5.657 \\
\hline
\end{tabular}


Table 2. (Continued)

MANATI OCTOBER 1984

\begin{tabular}{|c|c|c|c|c|c|c|c|}
\hline DAY & $\begin{array}{l}\text { MEAN } \\
(O F)\end{array}$ & $\begin{array}{l}\text { TEMPERATURE } \\
\text { MAX } \\
(O \mathrm{~F})\end{array}$ & $\begin{array}{l}\text { MIN } \\
(O F)\end{array}$ & $\begin{array}{l}\text { WII } \\
\text { VELOC } \\
\text { (MPH) }\end{array}$ & $\begin{array}{l}\text { ND } \\
\text { (MPD) }\end{array}$ & $\begin{array}{l}\text { DAILY } \\
\text { RAINFALL } \\
(\text { IN/DAY })\end{array}$ & $\begin{array}{l}\text { PAN } \\
\text { EVAPORATION } \\
\text { (IN/DAY) }\end{array}$ \\
\hline $\begin{array}{l}1 \\
2 \\
3 \\
4 \\
5 \\
5 \\
7 \\
8 \\
9 \\
10 \\
11 \\
12 \\
13 \\
14 \\
15 \\
16 \\
17 \\
18 \\
19 \\
20 \\
21 \\
22 \\
23 \\
24 \\
25 \\
26 \\
27 \\
28 \\
29 \\
30 \\
31\end{array}$ & $\begin{array}{l}79.3 \\
79.8 \\
79.5 \\
78.9 \\
78.9 \\
78.9 \\
78.9 \\
78.9 \\
78.3 \\
80.0 \\
79.5 \\
79.5 \\
79.5 \\
79.5 \\
79.5 \\
79.0 \\
77.8 \\
78.2 \\
78.2 \\
78.2 \\
78.2 \\
78.5 \\
79.2 \\
79.2 \\
79.2 \\
79.8 \\
79.6 \\
79.6 \\
79.3 \\
77.9 \\
76.5\end{array}$ & $\begin{array}{l}90.0 \\
90.5 \\
90.0 \\
89.8 \\
89.8 \\
89.8 \\
89.8 \\
89.8 \\
89.5 \\
89.5 \\
90.3 \\
90.3 \\
90.3 \\
90.3 \\
90.3 \\
91.0 \\
87.5 \\
89.3 \\
89.3 \\
89.3 \\
89.3 \\
91.0 \\
91.8 \\
91.8 \\
91.8 \\
92.5 \\
90.3 \\
90.3 \\
88.0 \\
87.0 \\
86.0\end{array}$ & $\begin{array}{l}68.5 \\
69.0 \\
69.0 \\
68.0 \\
68.0 \\
68.0 \\
68.0 \\
68.0 \\
67.0 \\
70.5 \\
68.8 \\
68.8 \\
68.8 \\
68.8 \\
68.8 \\
67.0 \\
68.0 \\
67.0 \\
67.0 \\
67.0 \\
67.0 \\
66.0 \\
66.5 \\
66.5 \\
66.5 \\
67.0 \\
68.8 \\
68.8 \\
70.5 \\
68.8 \\
67.0\end{array}$ & $\begin{array}{l}0.43 \\
2.23 \\
0.41 \\
0.41 \\
0.41 \\
0.41 \\
0.41 \\
0.41 \\
0.32 \\
0.65 \\
0.65 \\
0.65 \\
0.65 \\
0.65 \\
0.65 \\
0.69 \\
1.10 \\
1.10 \\
1.10 \\
1.10 \\
1.10 \\
0.66 \\
0.66 \\
0.66 \\
0.66 \\
1.05 \\
1.05 \\
1.05 \\
0.94 \\
0.94 \\
1.09\end{array}$ & $\begin{array}{r}10.4 \\
53.7 \\
9.7 \\
9.7 \\
9.7 \\
9.7 \\
9.7 \\
9.7 \\
7.6 \\
15.7 \\
15.7 \\
15.7 \\
15.7 \\
15.7 \\
15.7 \\
16.7 \\
26.4 \\
26.4 \\
26.4 \\
26.4 \\
26.4 \\
15.9 \\
15.9 \\
15.9 \\
15.9 \\
25.2 \\
25.2 \\
25.2 \\
22.6 \\
22.6 \\
26.1\end{array}$ & $\begin{array}{l}0.33 \\
0.03 \\
0.46 \\
0.46 \\
0.46 \\
0.46 \\
0.46 \\
0.46 \\
0.05 \\
0.01 \\
0.01 \\
0.01 \\
0.00 \\
0.00 \\
0.00 \\
0.00 \\
0.28 \\
0.28 \\
0.28 \\
0.27 \\
0.27 \\
0.00 \\
0.00 \\
0.00 \\
0.00 \\
0.10 \\
0.10 \\
0.10 \\
0.03 \\
0.02 \\
0.00\end{array}$ & $\begin{array}{l}0.292 \\
0.077 \\
0.260 \\
0.260 \\
0.260 \\
0.260 \\
0.260 \\
0.260 \\
0.070 \\
0.168 \\
0.168 \\
0.168 \\
0.168 \\
0.168 \\
0.168 \\
0.163 \\
0.163 \\
0.163 \\
0.163 \\
0.163 \\
0.163 \\
0.158 \\
0.158 \\
0.158 \\
0.158 \\
0.144 \\
0.144 \\
0.144 \\
0.158 \\
0.158 \\
0.132\end{array}$ \\
\hline $\begin{array}{l}\text { MEAN } \\
\text { DAILY } \\
\text { VAIUE }\end{array}$ & 78.9 & 89.9 & 68.0 & 0.78 & 18.8 & 0.16 & 0.177 \\
\hline $\begin{array}{l}\text { STANDARD } \\
\text { DEVIATION }\end{array}$ & 0.741 & 1.35 & 1.12 & 0.373 & 8.98 & 0.181 & 0.052 \\
\hline $\begin{array}{l}\text { EXTREME } \\
\text { DAILY } \\
\text { VALUE }\end{array}$ & $\mathrm{NA}$ & 91.8 & 66.0 & $\mathrm{NA}$ & $\begin{array}{l}\text { MAX } \\
53.7 \\
\text { MIN } \\
7.6\end{array}$ & $\begin{array}{l}\text { MAX } \\
0.46 \\
\text { MIN } \\
0.00\end{array}$ & $\begin{array}{c}\text { MAX } \\
0.292 \\
\text { MIN } \\
0.070\end{array}$ \\
\hline $\begin{array}{l}\text { TOTAL } \\
\text { FOR THE } \\
\text { MONTH }\end{array}$ & $\mathrm{NA}$ & NA & NA & $\mathrm{NA}$ & 583.3 & 4.93 & 5.497 \\
\hline
\end{tabular}


Table 2. (Continued)

MANATI NOVEMBER 1984

\begin{tabular}{|c|c|c|c|c|c|c|c|}
\hline DAY & $\begin{array}{l}\text { MEAN } \\
(O F)\end{array}$ & $\begin{array}{l}\text { TEMPERATURE } \\
\text { MAX } \\
(\circ \mathrm{F})\end{array}$ & $\begin{array}{l}\text { MIN } \\
\left({ }^{\circ} \mathrm{F}\right)\end{array}$ & $\begin{array}{r}W I \\
\text { VELO } \\
(\mathrm{MPH})\end{array}$ & $\begin{array}{l}\text { ND } \\
\text { CITY } \\
\text { (MPD) }\end{array}$ & $\begin{array}{c}\text { DAILY } \\
\text { RAINFALL } \\
\text { (IN/DAY })\end{array}$ & $\begin{array}{c}\text { PAN } \\
\text { EVAPORATION } \\
\text { (IN/DAY) }\end{array}$ \\
\hline $\begin{array}{l}1 \\
2 \\
3 \\
4 \\
5 \\
6 \\
7 \\
8 \\
9 \\
10 \\
11 \\
12 \\
13 \\
14 \\
15 \\
16 \\
17 \\
18 \\
19 \\
20 \\
21 \\
22 \\
23 \\
24 \\
25 \\
26 \\
27 \\
28 \\
29 \\
30\end{array}$ & $\begin{array}{l}78.0 \\
79.0 \\
78.4 \\
78.4 \\
77.8 \\
78.1 \\
78.1 \\
78.3 \\
79.5 \\
76.3 \\
76.3 \\
76.3 \\
74.0 \\
70.8 \\
78.0 \\
73.8 \\
74.7 \\
74.7 \\
75.5 \\
76.3 \\
76.8 \\
77.3 \\
77.8 \\
77.7 \\
77.7 \\
77.5 \\
75.5 \\
73.3 \\
72.0 \\
72.8\end{array}$ & $\begin{array}{l}86.5 \\
87.0 \\
86.5 \\
86.5 \\
86.0 \\
84.8 \\
84.8 \\
83.5 \\
84.0 \\
83.5 \\
83.5 \\
83.5 \\
83.0 \\
80.5 \\
81.0 \\
83.5 \\
84.3 \\
84.3 \\
85.0 \\
84.5 \\
84.5 \\
86.8 \\
89.0 \\
89.5 \\
89.5 \\
90.0 \\
82.0 \\
81.0 \\
80.5 \\
82.0\end{array}$ & $\begin{array}{l}69.5 \\
71.0 \\
70.3 \\
70.3 \\
69.5 \\
71.3 \\
71.3 \\
73.0 \\
75.0 \\
70.0 \\
70.0 \\
70.0 \\
65.0 \\
70.0 \\
75.0 \\
64.0 \\
65.0 \\
65.0 \\
66.0 \\
68.0 \\
69.0 \\
67.8 \\
66.5 \\
65.8 \\
65.8 \\
65.0 \\
69.0 \\
65.5 \\
63.5 \\
63.5\end{array}$ & $\begin{array}{l}1.10 \\
0.62 \\
0.62 \\
0.62 \\
2.18 \\
2.18 \\
2.18 \\
2.13 \\
2.13 \\
2.13 \\
2.13 \\
2.13 \\
1.14 \\
2.75 \\
2.84 \\
1.79 \\
1.79 \\
1.79 \\
1.53 \\
1.97 \\
1.95 \\
1.95 \\
1.08 \\
1.08 \\
1.08 \\
1.28 \\
1.50 \\
1.17 \\
1.79 \\
1.26\end{array}$ & $\begin{array}{l}26.5 \\
14.9 \\
14.9 \\
14.9 \\
52.3 \\
52.3 \\
52.3 \\
51.2 \\
51.2 \\
51.2 \\
51.2 \\
51.2 \\
27.3 \\
66.0 \\
68.0 \\
43.0 \\
43.0 \\
43.0 \\
36.8 \\
47.3 \\
46.7 \\
46.7 \\
26.0 \\
26.0 \\
26.0 \\
30.8 \\
36.1 \\
28.1 \\
42.9 \\
30.3\end{array}$ & $\begin{array}{l}0.01 \\
0.39 \\
0.39 \\
0.38 \\
0.25 \\
0.25 \\
0.24 \\
0.08 \\
0.02 \\
0.02 \\
0.02 \\
0.02 \\
1.13 \\
0.40 \\
0.00 \\
0.23 \\
0.22 \\
0.22 \\
0.01 \\
0.00 \\
0.03 \\
0.03 \\
0.00 \\
0.00 \\
0.00 \\
0.20 \\
0.01 \\
0.01 \\
0.01 \\
0.31\end{array}$ & $\begin{array}{l}0.109 \\
0.064 \\
0.064 \\
0.064 \\
0.071 \\
0.071 \\
0.071 \\
0.182 \\
0.194 \\
0.194 \\
0.194 \\
0.194 \\
0.052 \\
0.476 \\
0.259 \\
0.166 \\
0.166 \\
0.166 \\
0.119 \\
0.178 \\
0.178 \\
0.178 \\
0.131 \\
0.131 \\
0.131 \\
0.146 \\
0.114 \\
0.140 \\
0.146 \\
0.079\end{array}$ \\
\hline $\begin{array}{l}\text { MEAN } \\
\text { DAILY } \\
\text { VALUE }\end{array}$ & 74.0 & 84.7 & 68.4 & 1.66 & 39.9 & 0.15 & 0.148 \\
\hline $\begin{array}{l}\text { STANDARD } \\
\text { DEVIATION }\end{array}$ & 3.21 & 2.60 & 7.33 & 0.585 & 14.0 & 0.229 & 0.080 \\
\hline $\begin{array}{l}\text { EXTREME } \\
\text { DAILY } \\
\text { VALUE }\end{array}$ & NA & 90.0 & 63.5 & $\mathrm{NA}$ & $\begin{array}{l}\text { MAX } \\
52.3 \\
\text { MIN } \\
14.9\end{array}$ & $\begin{array}{l}\text { MAX } \\
1.13 \\
\text { MIN } \\
0.00\end{array}$ & $\begin{array}{c}\text { MAX } \\
0.259 \\
\text { MIN } \\
0.052\end{array}$ \\
\hline $\begin{array}{l}\text { TOTAL } \\
\text { FOR THE } \\
\text { MONTH }\end{array}$ & $\mathrm{NA}$ & NA & $\mathrm{NA}$ & $\mathrm{NA}$ & 1197.0 & 4.57 & 4.428 \\
\hline
\end{tabular}


Table 2. (Continued)

MANATI DECEMBER 1984

\begin{tabular}{|c|c|c|c|c|c|c|c|}
\hline DAY & $\begin{array}{l}\text { MEAN } \\
(O F)\end{array}$ & $\begin{array}{l}\text { TEMPERATURE } \\
\text { MAX } \\
\text { (OF) }\end{array}$ & $\begin{array}{l}\text { MIN } \\
(O F)\end{array}$ & $\begin{array}{l}\text { WII } \\
\text { VELOC } \\
(M P H)\end{array}$ & $\begin{array}{l}\text { ND } \\
\text { CITY } \\
\text { (MPD) }\end{array}$ & $\begin{array}{l}\text { DAIIY } \\
\text { RAINFALL } \\
(\text { IN/DAY })\end{array}$ & $\begin{array}{c}\text { PAN } \\
\text { EJAPORATION } \\
: \text { IN/ DAY) }\end{array}$ \\
\hline $\begin{array}{l}1 \\
2 \\
3 \\
4 \\
5 \\
6 \\
7 \\
8 \\
9 \\
10 \\
11 \\
12 \\
13 \\
14 \\
15 \\
16 \\
17 \\
18 \\
19 \\
20 \\
21 \\
22 \\
23 \\
24 \\
25 \\
26 \\
27 \\
28 \\
29 \\
30 \\
31\end{array}$ & $\begin{array}{l}72.8 \\
72.8 \\
72.8 \\
73.7 \\
74.5 \\
76.3 \\
74.5 \\
74.7 \\
74.7 \\
74.7 \\
74.8 \\
73.3 \\
74.3 \\
73.8 \\
73.8 \\
73.8 \\
73.8 \\
73.3 \\
72.3 \\
72.3 \\
75.5 \\
74.7 \\
74.7 \\
74.7 \\
74.7 \\
74.7 \\
73.8 \\
72.7 \\
72.7 \\
72.7 \\
72.7\end{array}$ & $\begin{array}{l}82.0 \\
82.0 \\
82.0 \\
82.3 \\
82.5 \\
83.0 \\
83.0 \\
83.0 \\
83.0 \\
83.0 \\
83.0 \\
80.5 \\
84.0 \\
81.0 \\
81.0 \\
81.0 \\
81.0 \\
78.0 \\
78.5 \\
78.0 \\
79.0 \\
80.3 \\
80.3 \\
80.3 \\
80.3 \\
80.3 \\
81.5 \\
80.8 \\
80.8 \\
80.8 \\
80.8\end{array}$ & $\begin{array}{l}63.5 \\
63.5 \\
63.5 \\
64.0 \\
66.5 \\
69.5 \\
66.0 \\
66.3 \\
66.3 \\
66.3 \\
66.5 \\
66.0 \\
64.5 \\
64.0 \\
64.0 \\
64.0 \\
64.0 \\
63.5 \\
66.0 \\
66.5 \\
72.0 \\
69.0 \\
69.0 \\
69.0 \\
69.0 \\
69.0 \\
66.0 \\
64.5 \\
64.5 \\
64.5 \\
64.5\end{array}$ & $\begin{array}{l}1.25 \\
1.26 \\
1.69 \\
1.69 \\
1.40 \\
1.65 \\
1.39 \\
1.39 \\
1.39 \\
1.39 \\
0.84 \\
0.88 \\
2.39 \\
2.39 \\
2.39 \\
2.39 \\
2.39 \\
2.33 \\
0.91 \\
1.47 \\
1.74 \\
1.74 \\
1.74 \\
1.74 \\
1.74 \\
1.74 \\
1.79 \\
1.79 \\
1.79 \\
1.79 \\
1.79\end{array}$ & $\begin{array}{l}30.3 \\
30.3 \\
40.7 \\
40.7 \\
33.6 \\
39.7 \\
33.8 \\
33.8 \\
33.8 \\
33.8 \\
20.2 \\
21.0 \\
57.4 \\
57.4 \\
57.4 \\
57.4 \\
57.4 \\
55.8 \\
21.8 \\
35.2 \\
41.7 \\
41.7 \\
41.7 \\
41.7 \\
41.7 \\
41.7 \\
43.0 \\
43.0 \\
43.0 \\
43.0 \\
43.0\end{array}$ & $\begin{array}{l}0.30 \\
0.30 \\
0.11 \\
0.10 \\
0.06 \\
0.01 \\
0.22 \\
0.22 \\
0.22 \\
0.22 \\
0.07 \\
0.00 \\
0.19 \\
0.19 \\
0.19 \\
0.18 \\
0.18 \\
0.01 \\
0.06 \\
0.00 \\
0.14 \\
0.14 \\
0.13 \\
0.13 \\
0.13 \\
0.13 \\
0.49 \\
0.48 \\
0.48 \\
0.48 \\
0.48\end{array}$ & $\begin{array}{l}0.079 \\
0.079 \\
0.163 \\
0.163 \\
0.155 \\
0.145 \\
0.147 \\
0.1477 \\
0.147 \\
0.147 \\
0.055 \\
0.111 \\
0.154 \\
0.154 \\
0.154 \\
0.154 \\
0.154 \\
0.175 \\
0.090 \\
0.139 \\
0.125 \\
0.125 \\
0.125 \\
0.125 \\
0.125 \\
0.125 \\
0.110 \\
0.110 \\
0.110 \\
0.110 \\
0.110\end{array}$ \\
\hline $\begin{array}{l}\text { MEAN } \\
\text { DAILY } \\
\text { VALUE }\end{array}$ & 73.9 & 81.2 & 66.0 & 1.69 & 40.5 & 0.19 & 0.130 \\
\hline $\begin{array}{l}\text { STANDARD } \\
\text { DEVIATION }\end{array}$ & 0.991 & 1.50 & 2.20 & 0.432 & 10.3 & 0.147 & 0.028 \\
\hline $\begin{array}{l}\text { EXTREME } \\
\text { DAILY } \\
\text { VALUE }\end{array}$ & NA & 83.0 & 53.5 & NA & $\begin{array}{l}\text { MAX } \\
57.4 \\
M I N \\
20.2\end{array}$ & $\begin{array}{l}\text { MAX } \\
0.48 \\
\text { MIN } \\
0.00\end{array}$ & $\begin{array}{c}\text { MAX } \\
0.175 \\
\text { MIN } \\
0.055\end{array}$ \\
\hline $\begin{array}{l}\text { TOTAL } \\
\text { FOR THE } \\
\text { MONTH }\end{array}$ & NA & $\mathrm{NA}$ & NA & $\mathrm{NA}$ & 1255.5 & 6.03 & 4.022 \\
\hline
\end{tabular}


Table 2. (Contlnued)

MANATI JANUARY 1985

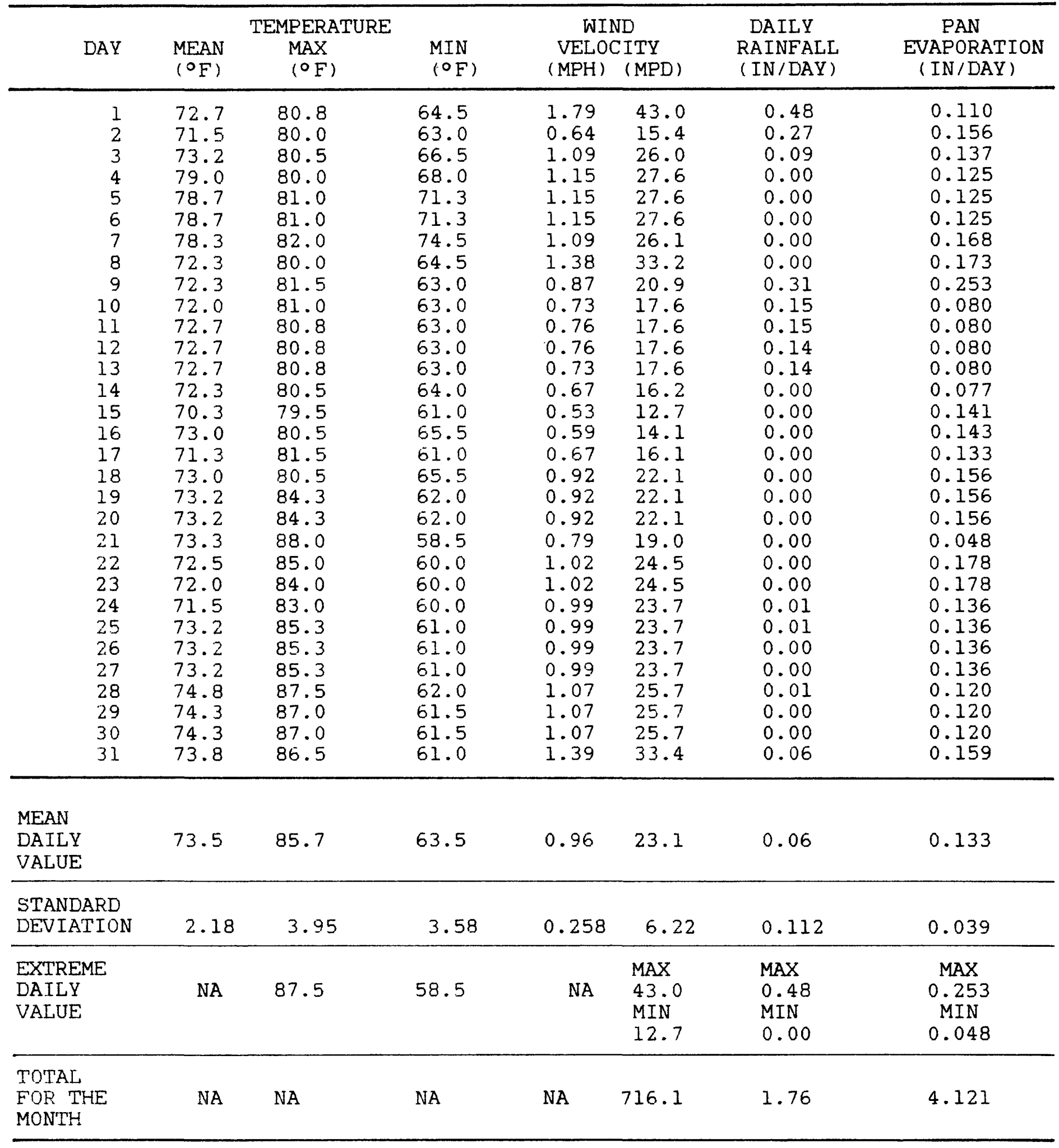


Table 2. (Continued)

MANATÍ

FEBRUARY 1985

\begin{tabular}{|c|c|c|c|c|c|c|c|}
\hline DAY & $\begin{array}{l}\text { MEAN } \\
\left({ }^{\circ} \mathrm{F}\right)\end{array}$ & $\begin{array}{l}\text { TEMPERATURE } \\
\text { MAX } \\
\left({ }^{\circ} \mathrm{F}\right)\end{array}$ & $\begin{array}{l}\text { MIN } \\
(O F)\end{array}$ & $\begin{array}{r}\text { WI } \\
\text { VELO } \\
\text { (MPH) }\end{array}$ & $\begin{array}{l}\text { ND } \\
\text { (MPD) }\end{array}$ & $\begin{array}{l}\text { DAILY } \\
\text { RAINFALL } \\
(\text { IN/DAY })\end{array}$ & $\begin{array}{c}\text { PAN } \\
\text { EVAPORATION } \\
(\text { IN /DAY })\end{array}$ \\
\hline $\begin{array}{l}1 \\
2 \\
3 \\
4 \\
5 \\
6 \\
7 \\
8 \\
9 \\
10 \\
11 \\
12 \\
13 \\
14 \\
15 \\
16 \\
17 \\
18 \\
19 \\
20 \\
21 \\
22 \\
23 \\
24 \\
25 \\
26 \\
27 \\
28\end{array}$ & $\begin{array}{l}73.7 \\
73.7 \\
73.7 \\
73.7 \\
73.7 \\
73.7 \\
73.5 \\
73.2 \\
73.2 \\
73.2 \\
73.2 \\
73.2 \\
73.2 \\
72.8 \\
74.4 \\
74.4 \\
74.4 \\
74.4 \\
74.4 \\
76.0 \\
72.8 \\
72.8 \\
72.8 \\
72.8 \\
72.8 \\
72.8 \\
75.4 \\
78.0\end{array}$ & $\begin{array}{l}86.8 \\
86.8 \\
86.8 \\
86.8 \\
86.8 \\
86.8 \\
87.0 \\
85.5 \\
85.5 \\
85.5 \\
85.5 \\
85.5 \\
85.5 \\
84.0 \\
87.3 \\
87.3 \\
87.3 \\
87.3 \\
87.3 \\
90.5 \\
84.0 \\
83.0 \\
83.0 \\
83.0 \\
83.0 \\
82.0 \\
81.5 \\
81.0\end{array}$ & $\begin{array}{l}60.5 \\
60.5 \\
60.5 \\
60.5 \\
60.5 \\
60.5 \\
60.0 \\
60.8 \\
60.8 \\
60.8 \\
60.8 \\
60.8 \\
60.8 \\
61.5 \\
61.5 \\
61.5 \\
61.5 \\
61.5 \\
61.5 \\
61.5 \\
61.5 \\
62.5 \\
62.5 \\
62.5 \\
62.5 \\
63.5 \\
64.3 \\
65.0\end{array}$ & $\begin{array}{l}1.39 \\
1.39 \\
1.39 \\
1.39 \\
1.39 \\
1.39 \\
1.05 \\
1.05 \\
1.05 \\
1.05 \\
1.05 \\
1.05 \\
1.05 \\
1.06 \\
1.06 \\
1.06 \\
1.06 \\
1.06 \\
1.06 \\
1.16 \\
1.24 \\
1.24 \\
1.24 \\
1.24 \\
1.24 \\
0.78 \\
0.78 \\
1.09\end{array}$ & $\begin{array}{l}33.4 \\
33.4 \\
33.4 \\
33.4 \\
33.4 \\
33.4 \\
25.2 \\
25.2 \\
25.2 \\
25.2 \\
25.2 \\
25.2 \\
25.2 \\
25.5 \\
25.5 \\
25.5 \\
25.5 \\
25.5 \\
25.5 \\
27.7 \\
29.7 \\
29.7 \\
29.7 \\
29.7 \\
29.7 \\
18.7 \\
18.7 \\
26.0\end{array}$ & $\begin{array}{l}0.06 \\
0.06 \\
0.06 \\
0.06 \\
0.05 \\
0.05 \\
0.02 \\
0.02 \\
0.01 \\
0.01 \\
0.01 \\
0.01 \\
0.01 \\
0.11 \\
0.11 \\
0.11 \\
0.11 \\
0.10 \\
0.10 \\
0.00 \\
0.31 \\
0.31 \\
0.31 \\
0.31 \\
0.31 \\
0.37 \\
0.36 \\
0.01\end{array}$ & $\begin{array}{l}0.159 \\
0.159 \\
0.159 \\
0.159 \\
0.159 \\
0.159 \\
0.143 \\
0.143 \\
0.143 \\
0.143 \\
0.143 \\
0.143 \\
0.143 \\
0.161 \\
0.161 \\
0.161 \\
0.161 \\
0.161 \\
0.161 \\
0.144 \\
0.187 \\
0.187 \\
0.187 \\
0.187 \\
0.187 \\
0.114 \\
0.114 \\
0.184\end{array}$ \\
\hline $\begin{array}{l}\text { MEAN } \\
\text { DAILY } \\
\text { VALUE }\end{array}$ & 73.8 & 85.4 & 61.5 & 1.15 & 27.5 & 0.12 & 0.156 \\
\hline $\begin{array}{l}\text { STANDARD } \\
\text { DEVIATION }\end{array}$ & 1.14 & 2.29 & 1.18 & 0.167 & 4.02 & 0.124 & 0.020 \\
\hline $\begin{array}{l}\text { EXTREME } \\
\text { DAILY } \\
\text { VALUE }\end{array}$ & $\mathrm{NA}$ & 87.3 & 60.0 & NA & $\begin{array}{l}\text { MAX } \\
33.4 \\
\text { MIN } \\
18.7\end{array}$ & $\begin{array}{l}\text { MAX } \\
0.37 \\
\text { MIN } \\
0.00\end{array}$ & $\begin{array}{c}\text { MAX } \\
0.187 \\
\text { MIN } \\
0.114\end{array}$ \\
\hline $\begin{array}{l}\text { TOTAL } \\
\text { FOR THE } \\
\text { MONTH }\end{array}$ & NA & NA & $\mathrm{NA}$ & $\mathrm{NA}$ & 770.0 & 3.36 & 4.412 \\
\hline
\end{tabular}


Tabie 2. (Continued)

MANATÍ

MARCH 1985

\begin{tabular}{|c|c|c|c|c|c|c|c|}
\hline DAY & $\begin{array}{l}\text { MEAN } \\
\left({ }^{\circ} \mathrm{F}\right)\end{array}$ & $\begin{array}{l}\text { MEPERA } \\
\text { (OF) }\end{array}$ & $\begin{array}{l}\text { MIN } \\
(\circ \mathrm{F})\end{array}$ & $\begin{array}{l}\text { WII } \\
\text { VELOC } \\
(\mathrm{MPH})\end{array}$ & $\begin{array}{l}\text { ID } \\
\text { ITY } \\
\text { (MPD) }\end{array}$ & $\begin{array}{l}\text { DAILY } \\
\text { RAINFALL } \\
\text { (IN/DAY) }\end{array}$ & $\begin{array}{c}\text { PAN } \\
\text { EVAPORATION } \\
\text { (IN/DAY) }\end{array}$ \\
\hline $\begin{array}{l}1 \\
2 \\
3 \\
4 \\
5 \\
6 \\
7 \\
8 \\
9 \\
10 \\
11 \\
12 \\
13 \\
14 \\
15 \\
16 \\
17 \\
18 \\
19 \\
20 \\
21 \\
22 \\
23 \\
24 \\
25 \\
26 \\
27 \\
28 \\
29 \\
30 \\
31\end{array}$ & $\begin{array}{l}75.9 \\
75.9 \\
75.9 \\
73.8 \\
72.4 \\
71.0 \\
71.2 \\
73.3 \\
73.8 \\
73.8 \\
73.8 \\
74.3 \\
73.7 \\
73.7 \\
73.0 \\
74.2 \\
74.2 \\
75.3 \\
73.7 \\
73.7 \\
72.0 \\
73.4 \\
73.4 \\
73.4 \\
73.4 \\
74.8 \\
73.6 \\
73.6 \\
72.3 \\
75.0 \\
75.0\end{array}$ & $\begin{array}{l}82.3 \\
82.3 \\
82.3 \\
83.5 \\
81.3 \\
79.0 \\
80.3 \\
81.5 \\
82.5 \\
82.5 \\
82.5 \\
83.5 \\
83.8 \\
83.8 \\
84.0 \\
85.8 \\
85.8 \\
87.5 \\
83.8 \\
83.8 \\
80.0 \\
82.0 \\
82.0 \\
82.0 \\
82.0 \\
84.0 \\
80.8 \\
80.8 \\
77.5 \\
80.3 \\
80.3\end{array}$ & $\begin{array}{l}64.5 \\
64.5 \\
64.5 \\
64.0 \\
63.5 \\
63.0 \\
64.0 \\
65.0 \\
65.0 \\
65.0 \\
65.0 \\
65.0 \\
63.5 \\
63.5 \\
62.0 \\
62.5 \\
62.5 \\
63.0 \\
63.5 \\
63.5 \\
64.0 \\
64.8 \\
64.8 \\
64.8 \\
64.8 \\
65.5 \\
66.3 \\
66.3 \\
67.0 \\
69.7 \\
69.7\end{array}$ & $\begin{array}{l}1.09 \\
1.09 \\
1.09 \\
0.99 \\
0.99 \\
0.80 \\
0.80 \\
1.02 \\
1.02 \\
1.02 \\
1.02 \\
1.01 \\
1.01 \\
1.01 \\
1.11 \\
1.11 \\
1.11 \\
0.79 \\
0.79 \\
0.79 \\
0.96 \\
0.96 \\
0.96 \\
0.90 \\
0.96 \\
1.64 \\
1.64 \\
1.64 \\
0.96 \\
0.96 \\
0.96\end{array}$ & $\begin{array}{l}26.0 \\
26.0 \\
26.0 \\
23.6 \\
23.6 \\
19.1 \\
19.1 \\
24.4 \\
24.4 \\
24.4 \\
24.4 \\
24.3 \\
24.3 \\
24.3 \\
26.6 \\
26.5 \\
26.6 \\
18.9 \\
18.9 \\
18.9 \\
23.0 \\
23.0 \\
23.0 \\
23.0 \\
23.0 \\
39.3 \\
39.3 \\
39.3 \\
23.0 \\
23.0 \\
23.0\end{array}$ & $\begin{array}{l}0.00 \\
0.00 \\
0.00 \\
0.08 \\
0.07 \\
0.75 \\
0.74 \\
0.04 \\
0.04 \\
0.04 \\
0.03 \\
0.00 \\
0.00 \\
0.00 \\
0.02 \\
0.01 \\
0.01 \\
0.32 \\
0.32 \\
0.31 \\
0.00 \\
0.00 \\
0.00 \\
0.00 \\
0.00 \\
1.20 \\
1.20 \\
1.20 \\
0.30 \\
0.29 \\
0.29\end{array}$ & $\begin{array}{l}0.184 \\
0.184 \\
0.184 \\
0.173 \\
0.173 \\
0.150 \\
0.150 \\
0.157 \\
0.157 \\
0.157 \\
0.157 \\
0.182 \\
0.182 \\
0.182 \\
0.172 \\
0.172 \\
0.172 \\
0.194 \\
0.194 \\
0.194 \\
0.185 \\
0.185 \\
0.185 \\
0.185 \\
0.185 \\
0.301 \\
0.301 \\
0.301 \\
0.171 \\
0.171 \\
0.171\end{array}$ \\
\hline $\begin{array}{l}\text { MEAN } \\
\text { DAILY } \\
\text { VALUE }\end{array}$ & 73.7 & 82.3 & 64.7 & 1.04 & 25.0 & 0.23 & 0.187 \\
\hline $\begin{array}{l}\text { STANDARD } \\
\text { DEVIATION }\end{array}$ & 1.19 & 2.02 & 1.73 & 0.218 & 13.2 & 0.373 & 0.039 \\
\hline $\begin{array}{l}\text { EXTREME } \\
\text { DAILY } \\
\text { VALUE }\end{array}$ & NA & 87.5 & 62.0 & NA & $\begin{array}{l}\text { MAX } \\
39.3 \\
\text { MIN } \\
18.9\end{array}$ & $\begin{array}{l}\text { MAX } \\
1.20 \\
\text { MIN } \\
0.00\end{array}$ & $\begin{array}{c}\text { MAX } \\
0.301 \\
\text { MIN } \\
0.150\end{array}$ \\
\hline $\begin{array}{l}\text { TOTAL } \\
\text { FOR THE } \\
\text { MONTH }\end{array}$ & NA & NA & NA & NA & 775.0 & 7.26 & 5.811 \\
\hline
\end{tabular}


Table 2. (Continued)

MANATI

APRIL 1985

\begin{tabular}{|c|c|c|c|c|c|c|c|}
\hline DAY & $\begin{array}{l}\text { MEAN } \\
\left({ }^{\circ} \mathrm{F}\right)\end{array}$ & $\begin{array}{l}\text { TEMPERATURE } \\
\text { MAX } \\
\left({ }^{\circ} \mathrm{F}\right)\end{array}$ & $\begin{array}{l}\text { MIN } \\
\left({ }^{\circ} \mathrm{F}\right)\end{array}$ & $\begin{array}{c}\text { WI } \\
\text { VELO } \\
(M P H)\end{array}$ & $\begin{array}{l}\text { ND } \\
\text { ITY } \\
\text { (MPD) }\end{array}$ & $\begin{array}{l}\text { DAILY } \\
\text { RAINFALL } \\
\text { (IN/DAY) }\end{array}$ & $\begin{array}{c}\text { PAN } \\
\text { EVAPORATION } \\
\text { (IN/DAY) }\end{array}$ \\
\hline $\begin{array}{l}1 \\
2 \\
3 \\
4 \\
5 \\
6 \\
7 \\
8 \\
9 \\
10 \\
11 \\
12 \\
13 \\
14 \\
15 \\
16 \\
17 \\
18 \\
19 \\
20 \\
21 \\
22 \\
23 \\
24 \\
25 \\
26 \\
27 \\
28 \\
29 \\
30\end{array}$ & $\begin{array}{l}73.3 \\
73.1 \\
72.8 \\
72.4 \\
72.4 \\
72.4 \\
72.4 \\
72.4 \\
72.4 \\
72.0 \\
75.0 \\
75.0 \\
75.0 \\
75.0 \\
75.0 \\
76.0 \\
76.0 \\
76.0 \\
77.0 \\
75.5 \\
75.5 \\
74.0 \\
74.0 \\
74.0 \\
74.0 \\
74.8 \\
74.8 \\
74.8 \\
74.8 \\
74.8\end{array}$ & $\begin{array}{l}83.0 \\
83.5 \\
84.0 \\
82.5 \\
82.5 \\
82.5 \\
82.5 \\
82.5 \\
82.5 \\
81.0 \\
85.5 \\
85.8 \\
85.8 \\
85.8 \\
86.0 \\
86.0 \\
86.0 \\
86.0 \\
86.0 \\
85.0 \\
85.0 \\
84.0 \\
84.0 \\
84.0 \\
83.0 \\
84.0 \\
84.0 \\
84.0 \\
84.0 \\
84.0\end{array}$ & $\begin{array}{l}63.5 \\
62.5 \\
61.5 \\
62.3 \\
62.3 \\
62.3 \\
62.3 \\
62.3 \\
62.3 \\
63.0 \\
64.5 \\
64.3 \\
64.3 \\
64.3 \\
64.0 \\
66.0 \\
66.0 \\
66.0 \\
68.0 \\
66.0 \\
66.0 \\
64.0 \\
64.0 \\
64.0 \\
65.0 \\
65.5 \\
65.5 \\
65.5 \\
65.5 \\
65.5\end{array}$ & $\begin{array}{l}0.85 \\
0.85 \\
0.96 \\
0.96 \\
0.96 \\
0.96 \\
0.96 \\
0.96 \\
0.96 \\
1.34 \\
1.33 \\
1.33 \\
1.33 \\
1.33 \\
1.32 \\
1.32 \\
1.32 \\
1.32 \\
1.23 \\
1.23 \\
1.23 \\
0.90 \\
0.90 \\
0.52 \\
1.46 \\
1.46 \\
1.46 \\
1.46 \\
1.46 \\
1.46\end{array}$ & $\begin{array}{l}20.5 \\
20.5 \\
23.1 \\
23.1 \\
23.1 \\
23.1 \\
23.1 \\
23.1 \\
23.1 \\
32.6 \\
32.0 \\
32.0 \\
32.0 \\
32.0 \\
31.8 \\
31.8 \\
31.8 \\
31.8 \\
29.5 \\
29.5 \\
29.5 \\
21.5 \\
21.5 \\
12.7 \\
35.1 \\
35.1 \\
35.1 \\
35.1 \\
35.1 \\
35.1\end{array}$ & $\begin{array}{l}0.00 \\
0.00 \\
0.05 \\
0.04 \\
0.04 \\
0.04 \\
0.04 \\
0.04 \\
0.04 \\
0.00 \\
0.05 \\
0.05 \\
0.05 \\
0.04 \\
0.00 \\
0.00 \\
0.00 \\
0.00 \\
0.55 \\
0.54 \\
0.54 \\
0.33 \\
0.33 \\
0.00 \\
0.04 \\
0.05 \\
0.05 \\
0.05 \\
0.05 \\
0.05\end{array}$ & $\begin{array}{l}0.219 \\
0.219 \\
0.209 \\
0.209 \\
0.209 \\
0.209 \\
0.209 \\
0.209 \\
0.209 \\
0.296 \\
0.228 \\
0.228 \\
0.228 \\
0.228 \\
0.235 \\
0.235 \\
0.235 \\
0.235 \\
0.243 \\
0.243 \\
0.243 \\
0.152 \\
0.152 \\
0.108 \\
0.223 \\
0.223 \\
0.223 \\
0.223 \\
0.223 \\
0.223\end{array}$ \\
\hline $\begin{array}{l}\text { MEAN } \\
\text { DAILY } \\
\text { VALUE }\end{array}$ & 74.2 & 84.1 & 66.5 & 1.21 & 29.1 & 0.10 & 0.225 \\
\hline $\begin{array}{l}\text { STANDARD } \\
\text { DEVIATION }\end{array}$ & 1.35 & 1.39 & 2.72 & 0.250 & 5.99 & 0.166 & 0.033 \\
\hline $\begin{array}{l}\text { EXTREME } \\
\text { DAILY } \\
\text { VALUE }\end{array}$ & NA & 86.0 & 51.5 & $\mathrm{NA}$ & $\begin{array}{l}\operatorname{MAX} \\
35.1 \\
\text { MIN } \\
12.7\end{array}$ & $\begin{array}{l}\text { MAX } \\
0.55 \\
\text { MIN } \\
0.00\end{array}$ & $\begin{array}{c}\text { MAX } \\
0.243 \\
\text { MIN } \\
0.108\end{array}$ \\
\hline $\begin{array}{l}\text { TOTAL } \\
\text { FOR THE } \\
\text { MONTH }\end{array}$ & NA & NA & NA & NA & 873.0 & 3.06 & 6.75 \\
\hline
\end{tabular}


Tabie 2. (Continued)

MANATÍ MAY 1985

\begin{tabular}{|c|c|c|c|c|c|c|c|}
\hline \multirow{2}{*}{ DAY } & \multicolumn{3}{|c|}{ TEMPERATURE } & \multicolumn{2}{|c|}{$\begin{array}{c}\text { WIND } \\
\text { VELOCITY }\end{array}$} & \multirow{2}{*}{$\begin{array}{c}\text { DAILY } \\
\text { RAINFALL } \\
\text { (IN/DAY) }\end{array}$} & \multirow{2}{*}{$\begin{array}{c}\text { PAN } \\
\text { EVAPORATION } \\
(\text { IN / DAY })\end{array}$} \\
\hline & $\begin{array}{l}\text { MEAN } \\
\left({ }^{\circ} \mathrm{F}\right)\end{array}$ & $\begin{array}{l}\text { MAX } \\
(O F)\end{array}$ & $\begin{array}{l}\text { MIN } \\
\left({ }^{\circ} \mathrm{F}\right)\end{array}$ & $\begin{array}{l}\text { VELO } \\
\text { (MPH) }\end{array}$ & (MPD) & & \\
\hline $\begin{array}{l}1 \\
2 \\
3 \\
4 \\
5 \\
6 \\
7 \\
8 \\
9 \\
10 \\
11 \\
12 \\
13 \\
14 \\
15 \\
16 \\
17\end{array}$ & $\begin{array}{l}74.8 \\
75.3 \\
75.8 \\
76.1 \\
76.1 \\
76.1 \\
76.3 \\
75.0 \\
75.5 \\
76.0 \\
77.0 \\
77.0 \\
77.0 \\
78.0 \\
77.8 \\
77.8 \\
77.5\end{array}$ & $\begin{array}{l}84.0 \\
85.0 \\
84.5 \\
85.5 \\
85.5 \\
85.5 \\
86.5 \\
82.5 \\
84.0 \\
85.5 \\
85.3 \\
85.3 \\
85.3 \\
85.0 \\
86.0 \\
86.0 \\
87.0\end{array}$ & $\begin{array}{l}65.5 \\
66.0 \\
67.0 \\
66.5 \\
66.5 \\
66.5 \\
66.0 \\
67.5 \\
66.0 \\
66.5 \\
68.8 \\
68.8 \\
68.8 \\
71.0 \\
69.5 \\
69.5 \\
68.0\end{array}$ & $\begin{array}{l}1.46 \\
1.40 \\
1.58 \\
1.58 \\
1.58 \\
1.58 \\
1.20 \\
1.94 \\
1.94 \\
1.72 \\
1.72 \\
1.72 \\
1.72 \\
0.95 \\
0.95 \\
0.95 \\
0.95\end{array}$ & $\begin{array}{l}35.1 \\
33.7 \\
38.0 \\
38.0 \\
38.0 \\
38.0 \\
28.8 \\
46.7 \\
46.7 \\
41.3 \\
41.3 \\
41.3 \\
41.3 \\
22.7 \\
22.7 \\
22.7 \\
22.7\end{array}$ & $\begin{array}{l}0.05 \\
0.92 \\
0.04 \\
0.04 \\
0.04 \\
0.03 \\
0.63 \\
0.02 \\
0.01 \\
0.04 \\
0.04 \\
0.04 \\
0.03 \\
0.58 \\
0.58 \\
0.57 \\
-.-.\end{array}$ & $\begin{array}{l}0.223 \\
0.223 \\
0.222 \\
0.222 \\
0.222 \\
0.222 \\
0.172 \\
0.155 \\
0.155 \\
0.231 \\
0.231 \\
0.231 \\
0.231 \\
0.162 \\
0.162 \\
0.162 \\
-.-1\end{array}$ \\
\hline $\begin{array}{l}\text { MEAN } \\
\text { DAILY } \\
\text { VALUE }\end{array}$ & --- & --- & --- & -- & --- & -- & --- \\
\hline $\begin{array}{l}\text { STANDARD } \\
\text { DEVIATION }\end{array}$ & --- & $-\cdots$ & --- & --- & --- & --- & --- \\
\hline $\begin{array}{l}\text { EXTREME } \\
\text { DAILY } \\
\text { VALUE }\end{array}$ & NA & -- & $-\cdots$ & NA & $\begin{array}{c}\text { MAX } \\
--- \\
\text { MIN } \\
\ldots-\end{array}$ & $\begin{array}{c}\text { MAX } \\
--- \\
\text { MIN } \\
---\end{array}$ & \begin{tabular}{c} 
MAX \\
\hdashline-- \\
MIN \\
---
\end{tabular} \\
\hline $\begin{array}{l}\text { TOTAL } \\
\text { FOR THE } \\
\text { MONTH }\end{array}$ & $\mathrm{NA}$ & $\mathrm{NA}$ & $\mathrm{NA}$ & NA & --- & --- & $\cdots$ \\
\hline
\end{tabular}


Table 3. Weather data and statistical analyses for the Arecibo weather statlon AUGUST 1983

\begin{tabular}{|c|c|c|c|c|c|c|c|}
\hline DAY & $\begin{array}{l}\text { MEAN } \\
(O F)\end{array}$ & $\begin{array}{c}\text { TEMPERA: } \\
\text { MAX } \\
(O F)\end{array}$ & $\begin{array}{l}\text { MIN } \\
(O F)\end{array}$ & $\begin{array}{c}\text { WII } \\
\text { VELOC } \\
\text { (MPH) }\end{array}$ & $\begin{array}{l}\text { ND } \\
\text { ITY } \\
\text { (MPD) }\end{array}$ & $\begin{array}{c}\text { DAILY } \\
\text { RAINFALL } \\
(\text { IN/DAY) }\end{array}$ & $\begin{array}{c}\text { PAN } \\
\text { EVAPORATION } \\
(\text { IN / DAY })\end{array}$ \\
\hline $\begin{array}{l}1 \\
2 \\
3 \\
4 \\
5 \\
6 \\
7 \\
8 \\
9 \\
10 \\
11 \\
12 \\
13 \\
14 \\
15 \\
16 \\
17 \\
18 \\
19 \\
20 \\
21 \\
22 \\
23 \\
24 \\
25 \\
26 \\
27 \\
28 \\
29 \\
30 \\
31\end{array}$ & $\begin{array}{l}--- \\
--- \\
--- \\
--- \\
78.0 \\
77.0 \\
77.0 \\
76.0 \\
76.0 \\
79.0 \\
76.5 \\
77.0 \\
77.0 \\
77.0 \\
77.0 \\
76.8 \\
76.5 \\
76.5 \\
79.0 \\
77.5 \\
77.5 \\
76.0 \\
74.5 \\
78.0 \\
76.0 \\
76.0 \\
76.0 \\
76.0 \\
76.0 \\
76.5 \\
76.0\end{array}$ & $\begin{array}{l}1-- \\
--- \\
-- \\
--- \\
91.0 \\
91.0 \\
91.0 \\
91.0 \\
90.0 \\
94.0 \\
91.0 \\
90.0 \\
91.5 \\
91.5 \\
93.0 \\
90.5 \\
88.0 \\
89.0 \\
94.0 \\
92.0 \\
92.0 \\
90.0 \\
89.0 \\
90.0 \\
89.0 \\
88.0 \\
88.5 \\
88.5 \\
89.0 \\
90.0 \\
89.0\end{array}$ & $\begin{array}{l}\ldots-. \\
-.- \\
-.- \\
65.0 \\
63.0 \\
63.0 \\
61.0 \\
62.0 \\
64.0 \\
62.0 \\
64.0 \\
62.5 \\
62.5 \\
61.0 \\
63.0 \\
65.0 \\
64.0 \\
64.0 \\
63.0 \\
63.0 \\
62.0 \\
65.0 \\
66.0 \\
63.0 \\
64.0 \\
63.5 \\
63.5 \\
63.0 \\
63.0 \\
63.0\end{array}$ & $\begin{array}{l}-.- \\
--- \\
--. \\
-.- \\
--- \\
--- \\
--- \\
0.41 \\
0.25 \\
0.25 \\
0.20 \\
0.20 \\
0.20 \\
0.20 \\
0.30 \\
0.30 \\
0.30 \\
2.11 \\
1.44 \\
1.44 \\
1.44 \\
2.36 \\
2.67 \\
2.22 \\
0.79 \\
0.53 \\
0.53 \\
0.53 \\
1.15 \\
0.66 \\
1.88\end{array}$ & $\begin{array}{c}--- \\
--- \\
--- \\
--- \\
--- \\
--- \\
--- \\
10.0 \\
6.1 \\
6.1 \\
4.8 \\
7.2 \\
7.2 \\
7.2 \\
7.2 \\
7.2 \\
7.2 \\
50.8 \\
34.6 \\
34.6 \\
34.6 \\
56.7 \\
64.0 \\
53.2 \\
19.1 \\
12.8 \\
12.8 \\
12.8 \\
27.5 \\
15.8 \\
45.1\end{array}$ & $\begin{array}{l}--- \\
--- \\
--- \\
--- \\
--- \\
--- \\
--- \\
--- \\
0.08 \\
0.07 \\
0.39 \\
0.38 \\
0.38 \\
0.38 \\
0.36 \\
0.36 \\
0.04 \\
0.00 \\
0.26 \\
0.26 \\
0.26 \\
0.00 \\
0.00 \\
0.30 \\
0.26 \\
0.31 \\
0.31 \\
0.30 \\
0.00 \\
0.04 \\
0.00\end{array}$ & 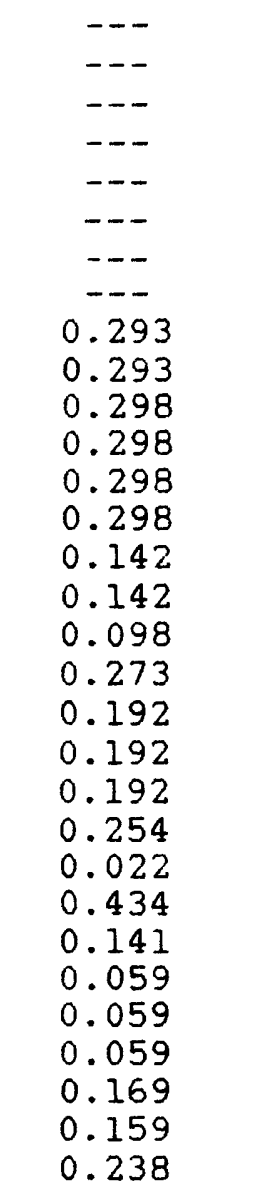 \\
\hline $\begin{array}{l}\text { MEAN } \\
\text { DAILY } \\
\text { VALUE }\end{array}$ & $\cdots$ & --- & --- & -- & $-\cdots$ & -- & -- \\
\hline $\begin{array}{l}\text { STANDARD } \\
\text { DEVIATION }\end{array}$ & $\cdots$ & --- & -- & --- & --- & --- & $-\cdots$ \\
\hline $\begin{array}{l}\text { EXTREME } \\
\text { DAILY } \\
\text { VALUE }\end{array}$ & NA & --- & -- & NA & $\begin{array}{l}\text { MAX } \\
--- \\
\text { MIN } \\
---\end{array}$ & $\begin{array}{l}\text { MAX } \\
--- \\
\text { MIN } \\
---\end{array}$ & $\begin{array}{l}\text { MAX } \\
--- \\
\text { MIN } \\
---\end{array}$ \\
\hline $\begin{array}{l}\text { TOTAL } \\
\text { FOR THE } \\
\text { MONTH }\end{array}$ & NA & NA & NA & NA & $-\cdots$ & --- & -- \\
\hline
\end{tabular}


Table 3. (Continued) ARECIBO SEPTEMBER 1983

\begin{tabular}{|c|c|c|c|c|c|c|c|}
\hline DAY & $\begin{array}{l}\text { MEAN } \\
(\mathrm{OF})\end{array}$ & $\begin{array}{l}\text { EMPERA } \\
\text { MAX } \\
(O \mathrm{~F})\end{array}$ & $\begin{array}{l}\text { MIN } \\
(\text { OF) }\end{array}$ & \multicolumn{2}{|c|}{$\begin{array}{c}\text { WIND } \\
\text { VELOCITY }\end{array}$} & $\begin{array}{l}\text { DAILY } \\
\text { RAINFALL } \\
\text { (IN/DAY) }\end{array}$ & $\begin{array}{c}\text { PAN } \\
\text { EVAPORATION } \\
(\text { IN /DAY })\end{array}$ \\
\hline $\begin{array}{r}1 \\
2 \\
3 \\
4 \\
5 \\
6 \\
7 \\
8 \\
9 \\
10 \\
11 \\
12 \\
13 \\
14 \\
15 \\
16 \\
17 \\
18 \\
19 \\
20 \\
21 \\
22 \\
23 \\
24 \\
25 \\
26 \\
27 \\
28 \\
29 \\
30\end{array}$ & $\begin{array}{l}77.5 \\
79.5 \\
77.8 \\
77.8 \\
77.8 \\
76.0 \\
76.5 \\
76.5 \\
74.0 \\
75.5 \\
75.5 \\
77.0 \\
77.5 \\
78.5 \\
79.0 \\
77.0 \\
78.3 \\
78.3 \\
79.5 \\
78.0 \\
77.0 \\
76.3 \\
75.5 \\
75.5 \\
75.5 \\
75.5 \\
77.5 \\
76.5 \\
76.5 \\
76.0\end{array}$ & $\begin{array}{l}89.0 \\
91.0 \\
90.5 \\
90.5 \\
90.5 \\
90.0 \\
89.0 \\
90.0 \\
92.0 \\
91.5 \\
91.5 \\
91.0 \\
91.0 \\
91.0 \\
92.0 \\
90.0 \\
90.5 \\
90.5 \\
91.0 \\
89.0 \\
88.0 \\
88.0 \\
88.0 \\
88.5 \\
88.5 \\
89.0 \\
93.0 \\
91.0 \\
90.0 \\
90.0\end{array}$ & $\begin{array}{l}66.0 \\
68.0 \\
65.0 \\
65.0 \\
65.0 \\
62.0 \\
62.0 \\
63.0 \\
60.0 \\
61.5 \\
61.5 \\
63.0 \\
64.0 \\
66.0 \\
66.0 \\
64.0 \\
66.0 \\
66.0 \\
68.0 \\
67.0 \\
66.0 \\
64.5 \\
63.0 \\
62.5 \\
62.5 \\
62.0 \\
62.0 \\
62.0 \\
63.0 \\
62.0\end{array}$ & $\begin{array}{l}1.96 \\
1.59 \\
1.59 \\
1.59 \\
1.59 \\
0.79 \\
0.33 \\
0.33 \\
1.51 \\
1.51 \\
1.51 \\
2.12 \\
2.37 \\
2.37 \\
0.62 \\
1.23 \\
1.23 \\
1.23 \\
1.65 \\
1.68 \\
0.27 \\
0.53 \\
0.59 \\
0.59 \\
0.59 \\
0.28 \\
0.64 \\
0.64 \\
1.32 \\
0.73\end{array}$ & $\begin{array}{r}47.0 \\
38.3 \\
38.3 \\
38.3 \\
38.3 \\
19.1 \\
8.0 \\
8.0 \\
36.2 \\
36.2 \\
36.2 \\
51.0 \\
57.0 \\
57.0 \\
14.9 \\
29.4 \\
29.4 \\
29.4 \\
39.7 \\
40.4 \\
6.5 \\
12.7 \\
14.2 \\
14.2 \\
14.2 \\
6.7 \\
15.4 \\
15.4 \\
31.6 \\
17.5\end{array}$ & $\begin{array}{l}0.00 \\
0.07 \\
0.06 \\
0.06 \\
0.06 \\
0.04 \\
0.07 \\
0.07 \\
0.04 \\
0.03 \\
0.03 \\
0.00 \\
0.04 \\
0.04 \\
0.00 \\
0.68 \\
0.68 \\
0.67 \\
0.21 \\
0.23 \\
0.00 \\
0.00 \\
0.14 \\
0.13 \\
0.13 \\
0.00 \\
0.05 \\
0.04 \\
0.00 \\
0.29\end{array}$ & $\begin{array}{l}0.257 \\
0.221 \\
0.221 \\
0.221 \\
0.221 \\
0.237 \\
0.193 \\
0.193 \\
0.251 \\
0.251 \\
0.251 \\
0.244 \\
0.275 \\
0.275 \\
0.142 \\
0.138 \\
0.138 \\
0.138 \\
0.071 \\
0.266 \\
0.251 \\
0.239 \\
0.153 \\
0.153 \\
0.153 \\
0.153 \\
0.106 \\
0.106 \\
0.357 \\
0.126\end{array}$ \\
\hline $\begin{array}{l}\text { MEAN } \\
\text { DAILY } \\
\text { VALUE }\end{array}$ & 76.9 & 90.2 & 63.9 & 1.17 & 28.0 & 0.13 & 0.200 \\
\hline $\begin{array}{l}\text { STANDARD } \\
\text { DEVIATION }\end{array}$ & 1.32 & 1.30 & 2.08 & 0.62 & 15.0 & 0.196 & 0.064 \\
\hline $\begin{array}{l}\text { EXTREME } \\
\text { DAILY } \\
\text { VALUE }\end{array}$ & NA & 93.0 & 60.0 & NA & $\begin{array}{l}\text { MAX } \\
57.0 \\
\text { MIN } \\
6.5\end{array}$ & $\begin{array}{l}\text { MAX } \\
0.68 \\
\text { MIN } \\
0.00\end{array}$ & $\begin{array}{c}\text { MAX } \\
0.357 \\
\text { MIN } \\
0.071\end{array}$ \\
\hline $\begin{array}{l}\text { TOTAL } \\
\text { FOR THE } \\
\text { MONTH }\end{array}$ & $\mathrm{NA}$ & NA & NA & NA & 840.0 & 3.86 & 6.013 \\
\hline
\end{tabular}


Table 3. (Continued)

ARECIBO OCTOBER 1983

\begin{tabular}{|c|c|c|c|c|c|c|c|}
\hline DAY & $\begin{array}{l}\text { MEAN } \\
\left({ }^{\circ} \mathrm{F}\right)\end{array}$ & $\begin{array}{c}\text { EMPERA } \\
\text { MAX } \\
(O \mathrm{~F})\end{array}$ & $\begin{array}{l}\text { MIN } \\
(O F)\end{array}$ & $\begin{array}{r}\text { WII } \\
\text { VELO } \\
(\mathrm{MPH})\end{array}$ & $\begin{array}{l}\text { JD } \\
\text { ITY } \\
\text { (MPD) }\end{array}$ & $\begin{array}{c}\text { DAILY } \\
\text { RAINFALL } \\
(\text { IN/DAY })\end{array}$ & $\begin{array}{c}\text { PAN } \\
\text { EVAPORATION } \\
\text { (IN / DAY) }\end{array}$ \\
\hline $\begin{array}{l}1 \\
2 \\
3 \\
4 \\
5 \\
6 \\
7 \\
8 \\
9 \\
10 \\
11 \\
12 \\
13 \\
14 \\
15 \\
16 \\
17 \\
18 \\
19 \\
20 \\
21 \\
22 \\
23 \\
24 \\
25 \\
26 \\
27 \\
28 \\
29 \\
30 \\
31\end{array}$ & $\begin{array}{l}76.0 \\
76.0 \\
76.0 \\
74.5 \\
75.0 \\
75.0 \\
75.5 \\
75.5 \\
75.5 \\
75.5 \\
75.5 \\
75.5 \\
75.5 \\
76.0 \\
76.0 \\
76.0 \\
76.0 \\
75.5 \\
75.0 \\
75.0 \\
75.5 \\
75.5 \\
75.5 \\
75.5 \\
76.5 \\
76.5 \\
76.5 \\
76.0 \\
75.0 \\
75.0 \\
74.0\end{array}$ & $\begin{array}{l}90.5 \\
90.5 \\
91.0 \\
87.0 \\
89.0 \\
89.0 \\
90.0 \\
89.5 \\
89.5 \\
89.5 \\
89.0 \\
89.5 \\
90.0 \\
89.0 \\
89.0 \\
89.0 \\
89.0 \\
89.0 \\
88.0 \\
88.0 \\
88.0 \\
88.5 \\
88.5 \\
89.0 \\
89.0 \\
91.0 \\
93.0 \\
92.0 \\
90.0 \\
90.0 \\
88.0\end{array}$ & $\begin{array}{l}61.5 \\
61.5 \\
61.0 \\
62.0 \\
61.0 \\
61.0 \\
61.0 \\
61.5 \\
61.5 \\
61.5 \\
62.0 \\
61.5 \\
61.0 \\
63.0 \\
63.0 \\
63.0 \\
63.0 \\
62.0 \\
62.0 \\
62.0 \\
63.0 \\
62.5 \\
62.5 \\
62.0 \\
64.0 \\
62.0 \\
60.0 \\
60.0 \\
60.0 \\
60.0 \\
60.0\end{array}$ & $\begin{array}{l}0.73 \\
0.73 \\
0.31 \\
0.86 \\
0.27 \\
1.09 \\
1.21 \\
1.21 \\
1.21 \\
1.21 \\
1.43 \\
1.32 \\
1.26 \\
0.68 \\
0.68 \\
0.68 \\
0.65 \\
0.21 \\
0.21 \\
0.44 \\
0.59 \\
0.59 \\
0.59 \\
0.59 \\
0.69 \\
0.52 \\
0.75 \\
0.75 \\
0.75 \\
0.75 \\
0.72\end{array}$ & $\begin{array}{r}17.5 \\
17.5 \\
7.6 \\
20.7 \\
6.6 \\
26.3 \\
29.0 \\
29.0 \\
29.0 \\
29.0 \\
34.3 \\
31.8 \\
30.2 \\
16.3 \\
16.3 \\
16.3 \\
15.6 \\
4.9 \\
4.9 \\
10.5 \\
14.2 \\
14.2 \\
14.2 \\
14.2 \\
16.6 \\
12.7 \\
18.0 \\
18.0 \\
18.0 \\
18.0 \\
17.4\end{array}$ & $\begin{array}{l}0.29 \\
0.29 \\
0.00 \\
0.00 \\
0.00 \\
0.00 \\
0.00 \\
0.00 \\
0.00 \\
0.00 \\
0.00 \\
0.46 \\
0.05 \\
0.15 \\
0.15 \\
0.14 \\
0.00 \\
0.30 \\
2.00 \\
0.70 \\
0.00 \\
0.00 \\
0.00 \\
0.00 \\
0.00 \\
0.00 \\
0.18 \\
0.18 \\
0.18 \\
0.17 \\
0.02\end{array}$ & $\begin{array}{l}0.126 \\
0.126 \\
0.164 \\
0.263 \\
0.230 \\
0.223 \\
0.088 \\
0.088 \\
0.088 \\
0.088 \\
0.126 \\
0.285 \\
0.096 \\
0.213 \\
0.213 \\
0.213 \\
0.202 \\
0.252 \\
0.252 \\
0.176 \\
0.199 \\
0.199 \\
0.199 \\
0.199 \\
0.170 \\
0.180 \\
0.161 \\
0.161 \\
0.161 \\
0.161 \\
0.184\end{array}$ \\
\hline $\begin{array}{l}\text { MEAN } \\
\text { DAILY } \\
\text { VALUE }\end{array}$ & 75.5 & 89.6 & 61.7 & 0.79 & 18.3 & 0.17 & 0.177 \\
\hline $\begin{array}{l}\text { STANDARD } \\
\text { DEVIATION }\end{array}$ & 0.560 & 1.23 & 1.03 & 0.328 & 7.83 & 0.371 & 0.054 \\
\hline $\begin{array}{l}\text { EXTREME } \\
\text { DAILY } \\
\text { VALUE }\end{array}$ & NA & 93.0 & 60.0 & $\mathrm{NA}$ & $\begin{array}{l}\text { MAX } \\
34.3 \\
\text { MIN } \\
4.9\end{array}$ & $\begin{array}{l}\text { MAX } \\
2.00 \\
\text { MIN } \\
0.00\end{array}$ & $\begin{array}{c}\text { MAX } \\
0.285 \\
\text { MIN } \\
0.088\end{array}$ \\
\hline $\begin{array}{l}\text { TOTAL } \\
\text { FOR THE } \\
\text { MONTH }\end{array}$ & NA & $\mathrm{NA}$ & NA & $\mathrm{NA}$ & 586.8 & 5.26 & 5.476 \\
\hline
\end{tabular}


Table 3. (Continued)

ARECIBO NOVEMBER 1983

\begin{tabular}{|c|c|c|c|c|c|c|c|}
\hline DAY & $\begin{array}{l}\text { MEAN } \\
\left({ }^{\circ} \mathrm{F}\right)\end{array}$ & $\begin{array}{l}\text { TEMPERATURE } \\
\text { MAX } \\
(\circ \mathrm{F})\end{array}$ & $\begin{array}{l}\text { MIN } \\
(\text { OF })\end{array}$ & $\begin{array}{r}\text { WII } \\
\text { VELOC } \\
(\text { MPH })\end{array}$ & $\begin{array}{l}\text { ND } \\
\text { (MPD) }\end{array}$ & $\begin{array}{l}\text { DAILY } \\
\text { RAINFALL } \\
\text { (IN/DAY) }\end{array}$ & $\begin{array}{c}\text { PAN } \\
\text { EVAPORATION } \\
\text { (IN/DAY) }\end{array}$ \\
\hline $\begin{array}{r}1 \\
2 \\
3 \\
4 \\
5 \\
6 \\
7 \\
8 \\
9 \\
10 \\
11 \\
12 \\
13 \\
14 \\
15 \\
16 \\
17 \\
18 \\
19 \\
20 \\
21 \\
22 \\
23 \\
24 \\
25 \\
26 \\
27 \\
28 \\
29 \\
30\end{array}$ & $\begin{array}{l}74.5 \\
74.5 \\
74.0 \\
70.0 \\
71.8 \\
71.8 \\
73.5 \\
73.5 \\
74.0 \\
74.0 \\
74.0 \\
74.5 \\
74.5 \\
75.0 \\
75.0 \\
74.5 \\
74.5 \\
74.5 \\
73.8 \\
73.8 \\
73.0 \\
73.0 \\
73.0 \\
72.3 \\
72.3 \\
72.3 \\
72.3 \\
71.5 \\
73.5 \\
71.0\end{array}$ & $\begin{array}{l}86.0 \\
87.0 \\
86.0 \\
77.0 \\
83.0 \\
83.0 \\
89.0 \\
89.0 \\
87.0 \\
87.0 \\
87.0 \\
88.5 \\
88.5 \\
90.0 \\
88.0 \\
89.0 \\
90.0 \\
90.0 \\
89.0 \\
89.0 \\
88.0 \\
88.5 \\
89.0 \\
87.5 \\
87.5 \\
87.5 \\
87.5 \\
86.0 \\
87.0 \\
81.0\end{array}$ & $\begin{array}{l}63.0 \\
62.0 \\
62.0 \\
63.0 \\
60.5 \\
60.5 \\
58.0 \\
58.0 \\
61.0 \\
61.0 \\
61.0 \\
60.5 \\
60.5 \\
60.0 \\
62.0 \\
60.0 \\
59.0 \\
59.0 \\
58.5 \\
58.5 \\
58.0 \\
58.5 \\
59.0 \\
58.0 \\
58.0 \\
58.0 \\
58.0 \\
57.0 \\
60.0 \\
61.0\end{array}$ & $\begin{array}{l}1.38 \\
0.00 \\
0.00 \\
0.40 \\
0.40 \\
0.40 \\
0.40 \\
0.40 \\
0.51 \\
0.51 \\
0.51 \\
0.51 \\
0.51 \\
0.51 \\
0.51 \\
0.51 \\
0.51 \\
1.35 \\
1.35 \\
1.35 \\
1.03 \\
1.03 \\
1.16 \\
1.16 \\
1.16 \\
1.16 \\
1.16 \\
1.90 \\
0.64 \\
1.29\end{array}$ & $\begin{array}{r}33.0 \\
0.0 \\
0.0 \\
9.6 \\
9.6 \\
9.6 \\
9.6 \\
9.6 \\
12.2 \\
12.2 \\
12.2 \\
12.2 \\
12.2 \\
12.2 \\
12.2 \\
12.2 \\
12.2 \\
32.3 \\
32.3 \\
32.3 \\
24.8 \\
24.8 \\
27.9 \\
27.9 \\
27.9 \\
27.9 \\
27.9 \\
45.7 \\
15.2 \\
31.0\end{array}$ & $\begin{array}{l}0.14 \\
0.00 \\
0.86 \\
0.20 \\
0.20 \\
0.20 \\
0.20 \\
0.00 \\
0.00 \\
0.00 \\
0.00 \\
0.00 \\
0.00 \\
0.00 \\
0.00 \\
0.00 \\
0.00 \\
0.01 \\
0.00 \\
0.00 \\
0.00 \\
0.00 \\
0.03 \\
0.03 \\
0.03 \\
0.02 \\
0.02 \\
0.10 \\
0.06 \\
0.00\end{array}$ & $\begin{array}{l}0.184 \\
0.192 \\
0.031 \\
0.225 \\
0.225 \\
0.225 \\
0.225 \\
0.225 \\
0.166 \\
0.166 \\
0.166 \\
0.166 \\
0.166 \\
0.166 \\
0.166 \\
0.166 \\
0.166 \\
0.167 \\
0.167 \\
0.167 \\
0.158 \\
0.158 \\
0.202 \\
0.202 \\
0.202 \\
0.202 \\
0.202 \\
0.202 \\
0.084 \\
0.166\end{array}$ \\
\hline $\begin{array}{l}\text { MEAN } \\
\text { DAILY } \\
\text { VALUE }\end{array}$ & 73.3 & 87.1 & 59.8 & 0.79 & 18.9 & 0.07 & 0.177 \\
\hline $\begin{array}{l}\text { STANDARD } \\
\text { DEVIATION }\end{array}$ & 1.25 & 2.78 & 1.64 & 0.450 & 11.0 & 0.160 & 0.040 \\
\hline $\begin{array}{l}\text { EXTREME } \\
\text { DAILY } \\
\text { VALUE }\end{array}$ & NA & 90.0 & 57.0 & NA & $\begin{array}{l}\text { MAX } \\
45.7 \\
\text { MIN } \\
0.0\end{array}$ & $\begin{array}{l}\text { MAX } \\
0.86 \\
\text { MIN } \\
0.00\end{array}$ & $\begin{array}{c}\text { MAX } \\
0.225 \\
\text { MIN } \\
0.031\end{array}$ \\
\hline $\begin{array}{l}\text { TOTAL } \\
\text { FOR THE } \\
\text { MONTH }\end{array}$ & $\mathrm{NA}$ & NA & NA & NA & 568.7 & 2.12 & 5.305 \\
\hline
\end{tabular}


Table 3. (Continued)

ARECIBO DECEMBER 1983

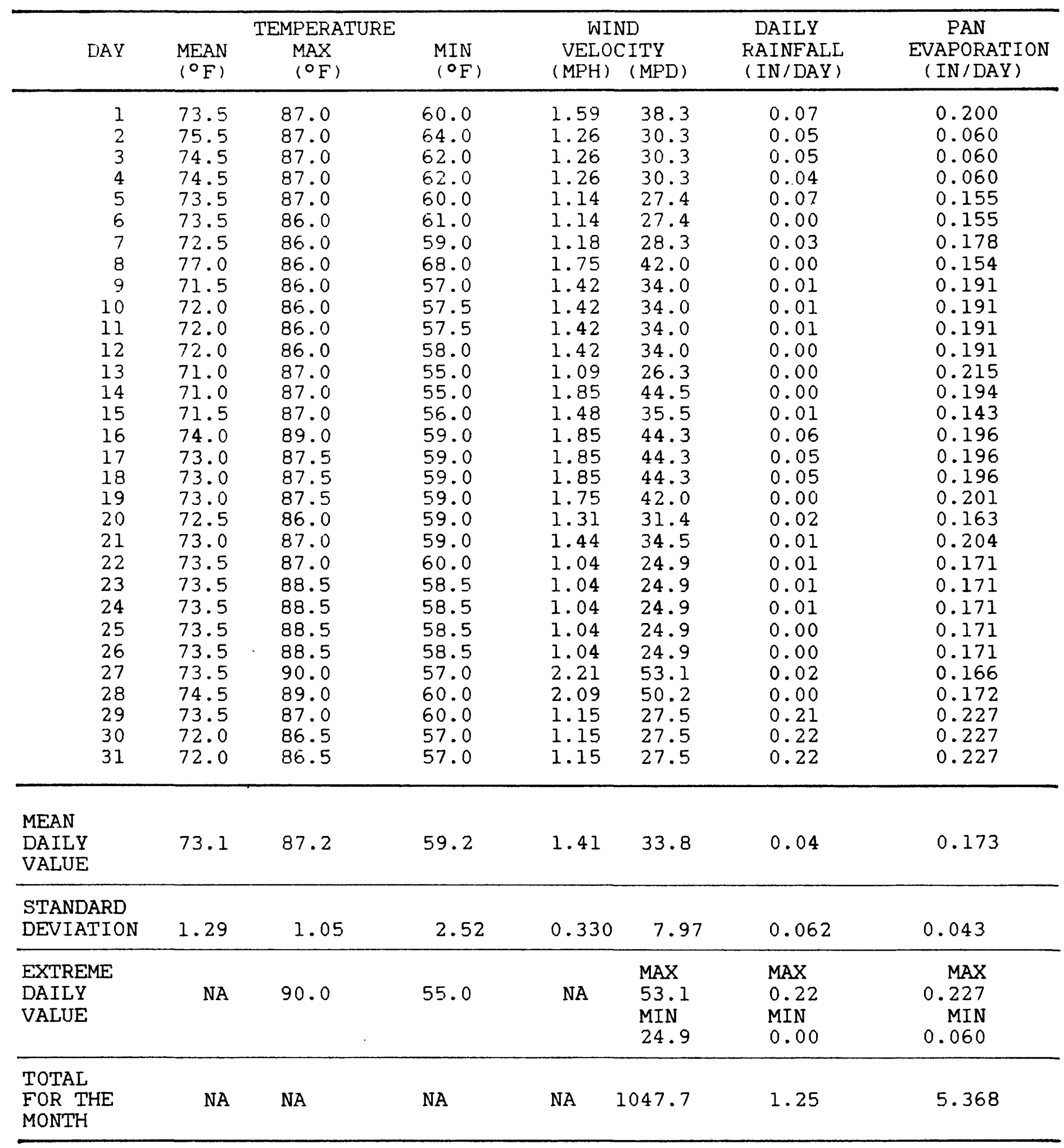


Table 3. (Continued)

ARECIBO JANUARY 1984

\begin{tabular}{|c|c|c|c|c|c|c|c|}
\hline DAY & $\begin{array}{l}\text { MEAN } \\
\left({ }^{\circ} \mathrm{F}\right)\end{array}$ & $\begin{array}{l}\text { EMPERA' } \\
\text { MAX } \\
\left({ }^{\circ} \mathrm{F}\right)\end{array}$ & $\begin{array}{l}\text { MIN } \\
(\circ \mathrm{F})\end{array}$ & \multicolumn{2}{|c|}{$\begin{array}{c}\text { WIND } \\
\text { VELOCITY } \\
\text { (MPH) (MPD) }\end{array}$} & $\begin{array}{l}\text { DAILY } \\
\text { RAINFALL } \\
\text { ( IN / DAY) }\end{array}$ & $\begin{array}{c}\text { PAN } \\
\text { EVAPORATION } \\
\text { (IN / DAY) }\end{array}$ \\
\hline $\begin{array}{l}1 \\
2 \\
3 \\
4 \\
5 \\
6 \\
7 \\
8 \\
9 \\
10 \\
11 \\
12 \\
13 \\
14 \\
15 \\
16 \\
17 \\
18 \\
19 \\
20 \\
21 \\
22 \\
23 \\
24 \\
25 \\
26 \\
27 \\
28 \\
29 \\
30 \\
31\end{array}$ & $\begin{array}{l}72.0 \\
72.0 \\
70.0 \\
70.5 \\
71.5 \\
71.5 \\
71.5 \\
71.5 \\
72.5 \\
72.0 \\
71.0 \\
71.0 \\
71.5 \\
72.0 \\
72.0 \\
72.0 \\
72.0 \\
72.0 \\
72.0 \\
72.0 \\
71.5 \\
71.5 \\
71.0 \\
71.5 \\
71.5 \\
71.5 \\
71.5 \\
71.5 \\
71.5 \\
71.5 \\
71.5\end{array}$ & $\begin{array}{l}86.5 \\
86.5 \\
86.0 \\
84.0 \\
86.0 \\
86.0 \\
86.0 \\
86.0 \\
88.0 \\
84.0 \\
84.0 \\
84.5 \\
85.0 \\
85.0 \\
85.0 \\
85.0 \\
85.0 \\
85.0 \\
85.0 \\
85.0 \\
85.5 \\
85.5 \\
86.0 \\
87.5 \\
87.5 \\
87.5 \\
87.5 \\
87.5 \\
87.5 \\
87.5 \\
87.5\end{array}$ & $\begin{array}{l}57.0 \\
57.0 \\
54.0 \\
57.0 \\
57.0 \\
57.0 \\
57.0 \\
57.0 \\
57.0 \\
60.0 \\
58.0 \\
58.0 \\
58.0 \\
58.5 \\
58.5 \\
58.5 \\
58.5 \\
58.5 \\
58.5 \\
59.0 \\
57.5 \\
57.5 \\
56.0 \\
55.5 \\
55.5 \\
55.5 \\
55.5 \\
55.5 \\
55.5 \\
55.5 \\
55.5\end{array}$ & $\begin{array}{l}1.15 \\
1.15 \\
0.80 \\
0.71 \\
0.83 \\
0.83 \\
0.83 \\
0.83 \\
1.25 \\
2.23 \\
1.53 \\
1.53 \\
1.62 \\
1.62 \\
1.62 \\
1.69 \\
1.69 \\
2.41 \\
2.41 \\
2.51 \\
2.51 \\
2.51 \\
0.54 \\
0.54 \\
0.54 \\
0.54 \\
0.54 \\
0.54 \\
0.54 \\
0.54 \\
0.54\end{array}$ & $\begin{array}{l}27.5 \\
27.5 \\
19.7 \\
17.1 \\
20.0 \\
20.0 \\
20.0 \\
20.0 \\
29.9 \\
53.4 \\
36.8 \\
36.8 \\
38.9 \\
38.9 \\
38.9 \\
40.5 \\
40.5 \\
57.9 \\
57.9 \\
60.4 \\
60.4 \\
60.4 \\
12.9 \\
12.9 \\
12.9 \\
12.9 \\
12.9 \\
12.9 \\
12.9 \\
12.9 \\
12.9\end{array}$ & $\begin{array}{l}0.22 \\
0.22 \\
0.00 \\
0.07 \\
0.07 \\
0.07 \\
0.06 \\
0.06 \\
0.16 \\
0.00 \\
0.00 \\
0.00 \\
0.00 \\
0.00 \\
0.00 \\
0.00 \\
0.00 \\
0.00 \\
0.00 \\
0.01 \\
0.01 \\
0.00 \\
0.02 \\
0.02 \\
0.02 \\
0.01 \\
0.01 \\
0.01 \\
0.01 \\
0.01 \\
0.01\end{array}$ & $\begin{array}{l}0.227 \\
0.227 \\
0.164 \\
0.125 \\
0.125 \\
0.125 \\
0.125 \\
0.125 \\
0.125 \\
0.125 \\
0.069 \\
0.069 \\
0.069 \\
0.069 \\
0.069 \\
0.069 \\
0.069 \\
0.144 \\
0.144 \\
0.277 \\
0.277 \\
0.277 \\
0.068 \\
0.068 \\
0.068 \\
0.068 \\
0.068 \\
0.068 \\
0.068 \\
0.008 \\
0.000\end{array}$ \\
\hline $\begin{array}{l}\text { MEAN } \\
\text { DAILY } \\
\text { VALUE }\end{array}$ & 71.6 & 85.9 & 57.1 & 1.26 & 30.3 & 0.07 & 0.115 \\
\hline $\begin{array}{l}\text { STANDARD } \\
\text { DEVIATION }\end{array}$ & 0.489 & 1.21 & 1.37 & 0.698 & 16.8 & 0.069 & 0.063 \\
\hline $\begin{array}{l}\text { EXTREME } \\
\text { DAILY } \\
\text { VALUE }\end{array}$ & $\mathrm{NA}$ & 87.5 & 54.0 & $\mathrm{NA}$ & $\begin{array}{l}\text { MAX } \\
60.4 \\
\text { MIN } \\
12.9\end{array}$ & $\begin{array}{l}\text { MAX } \\
0.22 \\
\text { MIN } \\
0.00\end{array}$ & $\begin{array}{c}\text { MAX } \\
0.277 \\
\text { MIN } \\
0.00\end{array}$ \\
\hline $\begin{array}{l}\text { TOTAL } \\
\text { FOR THE } \\
\text { MONTH }\end{array}$ & NA & NA & NA & NA & 939.0 & 2.13 & 3.565 \\
\hline
\end{tabular}


Table 3. (Continued)

ARECIBO

APRIL 1984

\begin{tabular}{|c|c|c|c|c|c|c|c|}
\hline DAY & $\begin{array}{l}\text { MEAN } \\
\left({ }^{\circ} \mathrm{F}\right)\end{array}$ & $\begin{array}{c}\text { CEMPERA } \\
\text { MAX } \\
(\circ \mathrm{F})\end{array}$ & $\begin{array}{l}\text { MIN } \\
(O F)\end{array}$ & \multicolumn{2}{|c|}{$\begin{array}{c}\text { WIND } \\
\text { VELOCITY }\end{array}$} & $\begin{array}{c}\text { DAILY } \\
\text { RAINFALL } \\
\text { ( IN/DAY) }\end{array}$ & $\begin{array}{c}\text { PAN } \\
\text { EVAPORATION } \\
(\text { IN/DAY })\end{array}$ \\
\hline $\begin{array}{r}1 \\
2 \\
3 \\
4 \\
5 \\
6 \\
7 \\
8 \\
9 \\
10 \\
11 \\
12 \\
13 \\
14 \\
15 \\
16 \\
17 \\
18 \\
19 \\
20 \\
21 \\
22 \\
23 \\
24 \\
25 \\
26 \\
27 \\
28 \\
29 \\
30\end{array}$ & $\begin{array}{l}---- \\
75.5 \\
76.0 \\
72.5 \\
72.5 \\
74.5 \\
74.0 \\
74.0 \\
74.0 \\
71.5 \\
73.5 \\
74.0 \\
76.0 \\
76.0 \\
76.0 \\
76.0 \\
74.0 \\
73.5 \\
73.0 \\
73.0 \\
73.0 \\
73.0 \\
73.0 \\
73.0 \\
73.5 \\
75.0 \\
75.5 \\
73.5 \\
73.5 \\
71.5\end{array}$ & $\begin{array}{l}---- \\
90.0 \\
89.0 \\
84.0 \\
88.0 \\
92.0 \\
91.5 \\
91.5 \\
91.0 \\
88.0 \\
90.0 \\
89.0 \\
91.0 \\
90.5 \\
90.5 \\
90.0 \\
89.0 \\
89.0 \\
89.0 \\
89.0 \\
89.0 \\
89.0 \\
89.0 \\
90.0 \\
90.0 \\
90.0 \\
90.0 \\
88.0 \\
88.0 \\
86.0\end{array}$ & $\begin{array}{l}---- \\
61.0 \\
63.0 \\
61.0 \\
57.0 \\
57.0 \\
57.0 \\
57.0 \\
57.0 \\
55.0 \\
57.0 \\
59.0 \\
61.0 \\
61.5 \\
61.5 \\
62.0 \\
59.0 \\
58.0 \\
57.5 \\
57.5 \\
57.5 \\
57.5 \\
57.0 \\
56.0 \\
58.0 \\
60.0 \\
61.0 \\
59.0 \\
59.0 \\
57.0\end{array}$ & $\begin{array}{l}2.35 \\
1.74 \\
1.27 \\
3.00 \\
1.94 \\
1.45 \\
1.45 \\
1.45 \\
3.06 \\
1.75 \\
1.49 \\
1.59 \\
3.04 \\
3.04 \\
3.04 \\
0.79 \\
2.45 \\
3.27 \\
4.69 \\
4.69 \\
4.69 \\
4.64 \\
3.21 \\
2.91 \\
2.79 \\
2.31 \\
4.42 \\
4.42 \\
4.42 \\
2.39\end{array}$ & $\begin{array}{r}56.5 \\
41.9 \\
30.5 \\
72.0 \\
46.6 \\
34.9 \\
34.9 \\
34.9 \\
73.5 \\
42.0 \\
35.7 \\
38.3 \\
72.8 \\
72.8 \\
72.8 \\
17.0 \\
58.7 \\
78.5 \\
112.6 \\
112.6 \\
112.6 \\
112.6 \\
77.2 \\
69.7 \\
67.0 \\
55.4 \\
106.1 \\
106.1 \\
106.1 \\
57.4\end{array}$ & $\begin{array}{l}0.00 \\
0.30 \\
0.00 \\
0.00 \\
0.00 \\
0.00 \\
0.00 \\
0.00 \\
0.00 \\
0.00 \\
0.00 \\
0.00 \\
0.10 \\
0.10 \\
0.10 \\
0.00 \\
0.00 \\
0.00 \\
0.00 \\
0.00 \\
0.00 \\
0.00 \\
0.00 \\
0.00 \\
0.00 \\
0.00 \\
0.00 \\
0.00 \\
0.00 \\
0.02\end{array}$ & $\begin{array}{l}0.428 \\
0.160 \\
0.293 \\
0.325 \\
0.252 \\
0.126 \\
0.126 \\
0.126 \\
0.126 \\
0.228 \\
0.290 \\
0.319 \\
0.326 \\
0.326 \\
0.326 \\
0.485 \\
0.274 \\
0.364 \\
0.503 \\
0.503 \\
0.503 \\
0.503 \\
0.296 \\
0.318 \\
0.335 \\
0.282 \\
0.136 \\
0.136 \\
0.136 \\
0.248\end{array}$ \\
\hline $\begin{array}{l}\text { MEAN } \\
\text { DAILY } \\
\text { VALUE }\end{array}$ & 74.0 & 89.3 & 58.6 & 2.79 & 67.0 & 0.02 & 0.293 \\
\hline $\begin{array}{l}\text { STANDARD } \\
\text { DEVIATION }\end{array}$ & 1.31 & 1.61 & 2.03 & 1.18 & 28.4 & 0.059 & 0.123 \\
\hline $\begin{array}{l}\text { EXTREME } \\
\text { DAILY } \\
\text { VALUE }\end{array}$ & $\mathrm{NA}$ & 92.0 & 56.0 & NA & $\begin{array}{c}\text { MAX } \\
112.6 \\
\text { MIN }\end{array}$ & $\begin{array}{l}\text { MAX } \\
0.30 \\
\text { MIN }\end{array}$ & $\begin{array}{c}\text { MAX } \\
0.503 \\
\text { MIN }\end{array}$ \\
\hline $\begin{array}{l}\text { TOTAL } \\
\text { FOR THE } \\
\text { MONTH }\end{array}$ & NA & $\mathrm{NA}$ & NA & NA & 2009.7 & 0.62 & 8.790 \\
\hline
\end{tabular}


Table 3. (Continued)

ARECIBO

MAY 1984

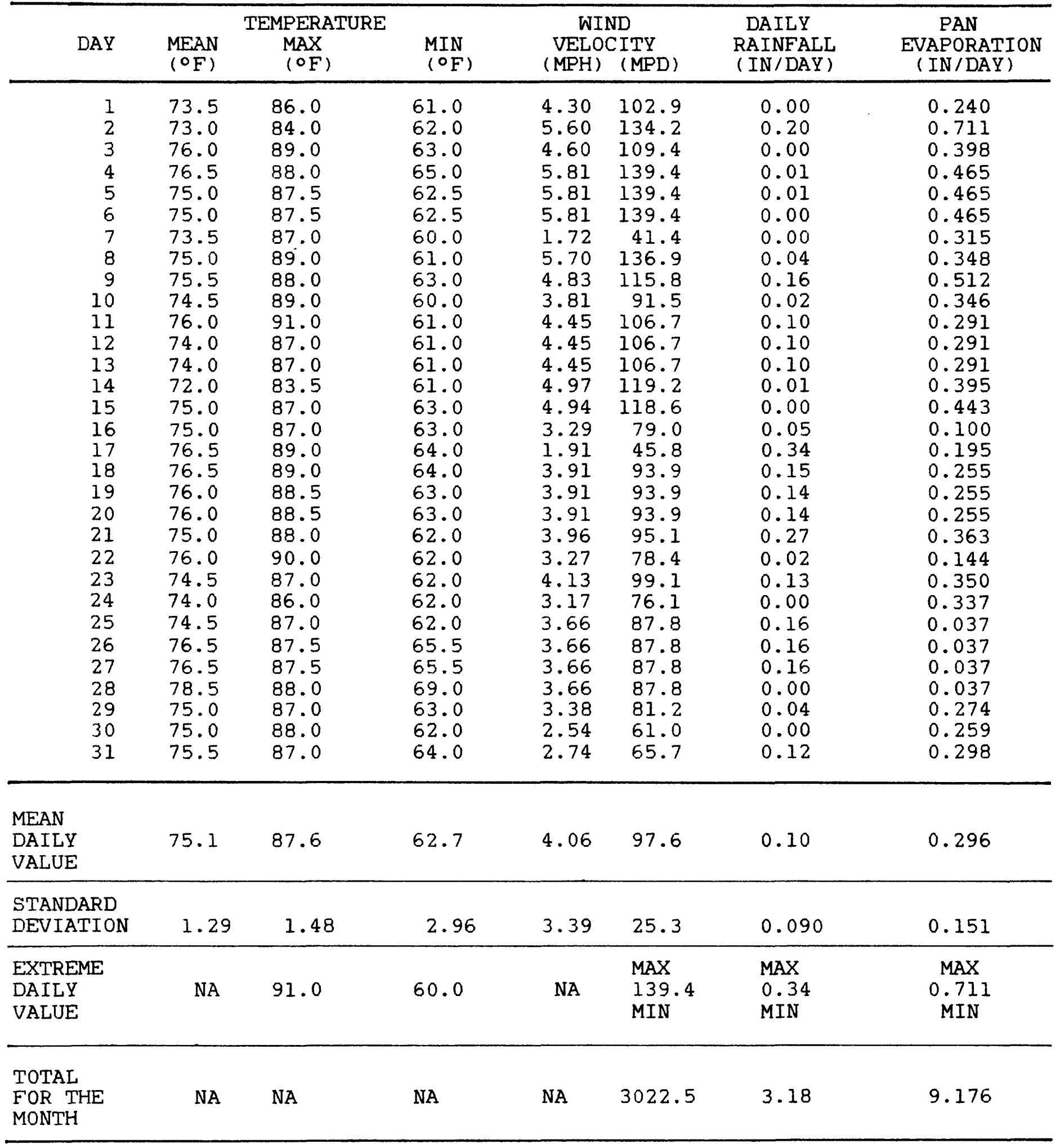


Table 3. (Continued)

ARECIBO JUNE 1984

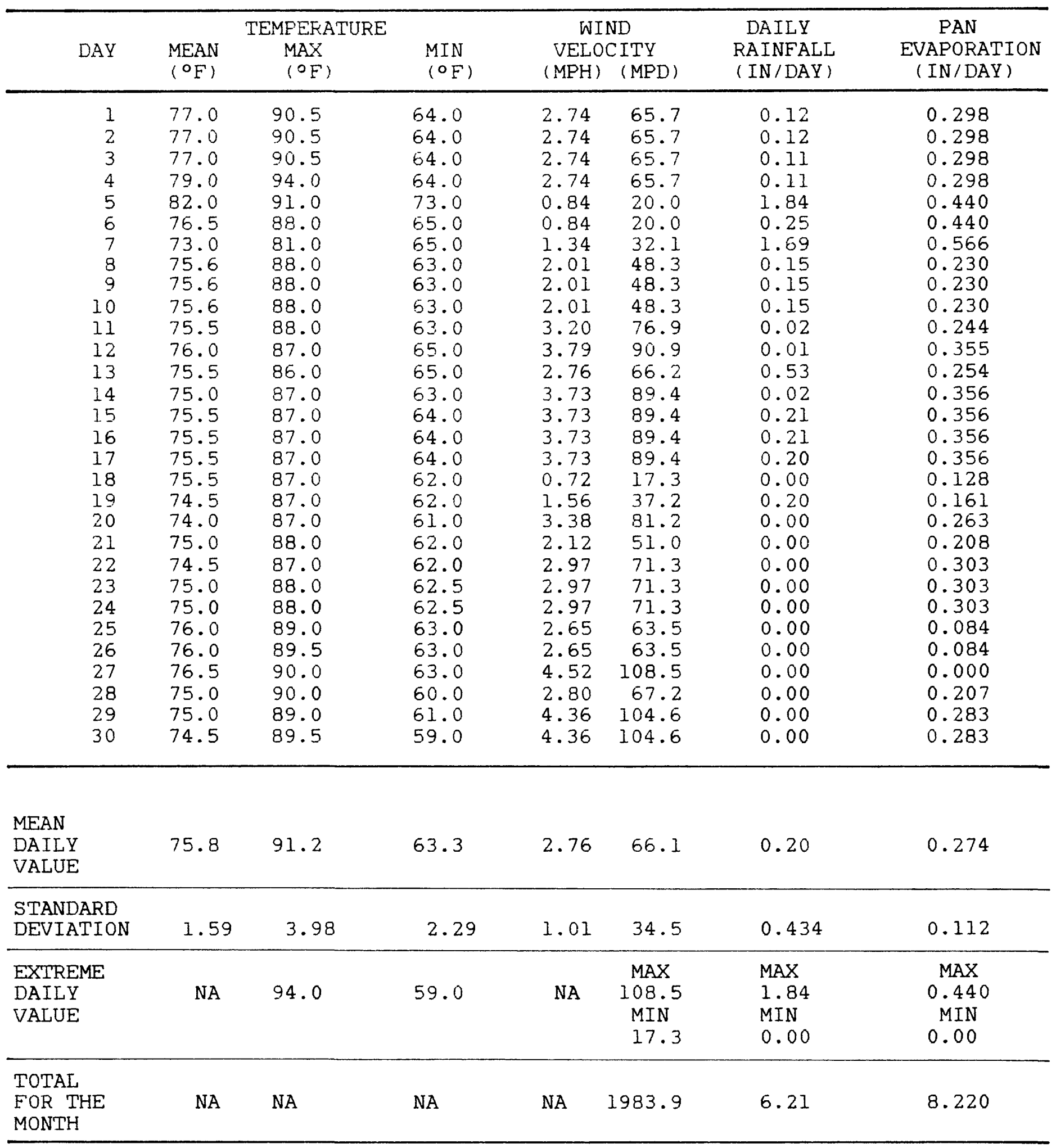


Table 3. (Continued)

ARECIBO JULY 1984

\begin{tabular}{|c|c|c|c|c|c|c|c|}
\hline DAY & $\begin{array}{l}\text { MEAN } \\
\left({ }^{\circ} \mathrm{F}\right)\end{array}$ & $\begin{array}{l}\text { TEMPERATURE } \\
\text { MAX } \\
\left({ }^{\circ} \mathrm{F}\right)\end{array}$ & $\begin{array}{l}\text { MIN } \\
(\circ \mathrm{F})\end{array}$ & $\begin{array}{r}\text { WII } \\
\text { VELOC } \\
(\mathrm{MPH})\end{array}$ & $\begin{array}{l}\text { ND } \\
\text { (MPD) }\end{array}$ & $\begin{array}{l}\text { DAILY } \\
\text { RAINFALL } \\
\text { (IN/DAY) }\end{array}$ & $\begin{array}{l}\text { PAN } \\
\text { EVAPORATION } \\
(\text { IN/DAY })\end{array}$ \\
\hline $\begin{array}{r}1 \\
2 \\
3 \\
4 \\
5 \\
6 \\
7 \\
8 \\
9 \\
10 \\
11 \\
12 \\
13 \\
14 \\
15 \\
16 \\
17 \\
18 \\
19 \\
20 \\
21 \\
22 \\
23 \\
24 \\
25 \\
26 \\
27 \\
28 \\
29 \\
30 \\
31\end{array}$ & $\begin{array}{l}74.5 \\
74.0 \\
74.5 \\
74.5 \\
75.0 \\
74.5 \\
76.0 \\
76.0 \\
77.5 \\
75.0 \\
75.5 \\
76.0 \\
76.5 \\
76.3 \\
76.3 \\
76.0 \\
75.8 \\
75.5 \\
75.0 \\
74.5 \\
75.0 \\
75.0 \\
75.5 \\
77.0 \\
78.5 \\
78.5 \\
75.0 \\
75.8 \\
75.8 \\
76.5 \\
78.0\end{array}$ & $\begin{array}{l}89.5 \\
90.0 \\
89.5 \\
89.5 \\
89.0 \\
88.0 \\
88.5 \\
88.5 \\
89.0 \\
88.0 \\
88.0 \\
88.0 \\
90.0 \\
90.0 \\
90.0 \\
90.0 \\
89.0 \\
88.0 \\
88.0 \\
88.0 \\
88.0 \\
88.0 \\
88.0 \\
88.0 \\
89.0 \\
89.0 \\
93.5 \\
92.3 \\
92.3 \\
91.0 \\
93.0\end{array}$ & $\begin{array}{l}59.0 \\
57.0 \\
59.0 \\
59.0 \\
61.0 \\
71.0 \\
68.5 \\
68.5 \\
66.0 \\
62.0 \\
63.0 \\
64.0 \\
63.0 \\
62.5 \\
62.5 \\
62.0 \\
62.5 \\
63.0 \\
62.0 \\
61.0 \\
62.0 \\
62.0 \\
63.0 \\
66.0 \\
68.0 \\
68.0 \\
64.5 \\
63.3 \\
63.3 \\
62.0 \\
63.0\end{array}$ & $\begin{array}{l}4.36 \\
2.15 \\
2.15 \\
2.15 \\
0.55 \\
3.59 \\
3.59 \\
3.59 \\
2.64 \\
2.77 \\
2.77 \\
4.11 \\
3.21 \\
3.21 \\
3.21 \\
3.74 \\
3.74 \\
2.71 \\
2.71 \\
2.45 \\
2.45 \\
2.45 \\
3.52 \\
6.24 \\
6.51 \\
3.68 \\
2.75 \\
2.75 \\
2.75 \\
2.55 \\
3.57\end{array}$ & $\begin{array}{r}104.6 \\
51.5 \\
51.5 \\
51.5 \\
13.1 \\
86.1 \\
86.1 \\
86.1 \\
63.3 \\
66.5 \\
66.5 \\
98.7 \\
76.9 \\
76.9 \\
76.9 \\
89.7 \\
89.7 \\
65.1 \\
65.1 \\
58.9 \\
58.9 \\
58.9 \\
84.4 \\
149.9 \\
156.1 \\
88.2 \\
66.0 \\
66.0 \\
66.0 \\
61.1 \\
85.9\end{array}$ & $\begin{array}{l}0.00 \\
0.43 \\
0.43 \\
0.42 \\
0.04 \\
0.00 \\
0.00 \\
0.00 \\
0.00 \\
0.01 \\
0.02 \\
0.00 \\
0.10 \\
0.09 \\
0.09 \\
0.00 \\
0.00 \\
0.00 \\
0.00 \\
0.02 \\
0.01 \\
0.01 \\
0.15 \\
0.01 \\
0.06 \\
0.00 \\
0.04 \\
0.03 \\
0.03 \\
0.00 \\
0.07\end{array}$ & $\begin{array}{l}0.283 \\
0.434 \\
0.434 \\
0.434 \\
0.203 \\
0.298 \\
0.298 \\
0.298 \\
0.340 \\
0.230 \\
0.230 \\
0.270 \\
0.270 \\
0.270 \\
0.270 \\
0.327 \\
0.327 \\
0.263 \\
0.263 \\
0.205 \\
0.205 \\
0.205 \\
0.225 \\
0.253 \\
0.398 \\
0.368 \\
0.270 \\
0.270 \\
0.270 \\
0.305 \\
0.347\end{array}$ \\
\hline $\begin{array}{l}\text { MEAN } \\
\text { DAILY } \\
\text { VALUE }\end{array}$ & 75.8 & 89.4 & 63.3 & 3.18 & 76.3 & 0.07 & 0.292 \\
\hline $\begin{array}{l}\text { STANDARD } \\
\text { DEVIATION }\end{array}$ & 1.16 & 1.55 & 3.07 & 3.26 & 26.6 & 0.124 & 0.066 \\
\hline $\begin{array}{l}\text { EXTREME } \\
\text { DAILY } \\
\text { VALUE }\end{array}$ & NA & 93.0 & 57.0 & NA & $\begin{array}{l}\text { MAX } \\
156.1 \\
\text { MIN } \\
13.1\end{array}$ & $\begin{array}{l}\text { MAX } \\
0.43 \\
\text { MIN } \\
0.00\end{array}$ & $\begin{array}{c}\text { MAX } \\
0.434 \\
\text { MIN } \\
0.205\end{array}$ \\
\hline $\begin{array}{l}\text { TOTAL } \\
\text { FOR THE } \\
\text { MONTH }\end{array}$ & $\mathrm{NA}$ & $\mathrm{NA}$ & NA & NA & 2366.1 & 2.11 & 9.063 \\
\hline
\end{tabular}


Table 3. (Continued)

ARECIBO AUGUST 1984

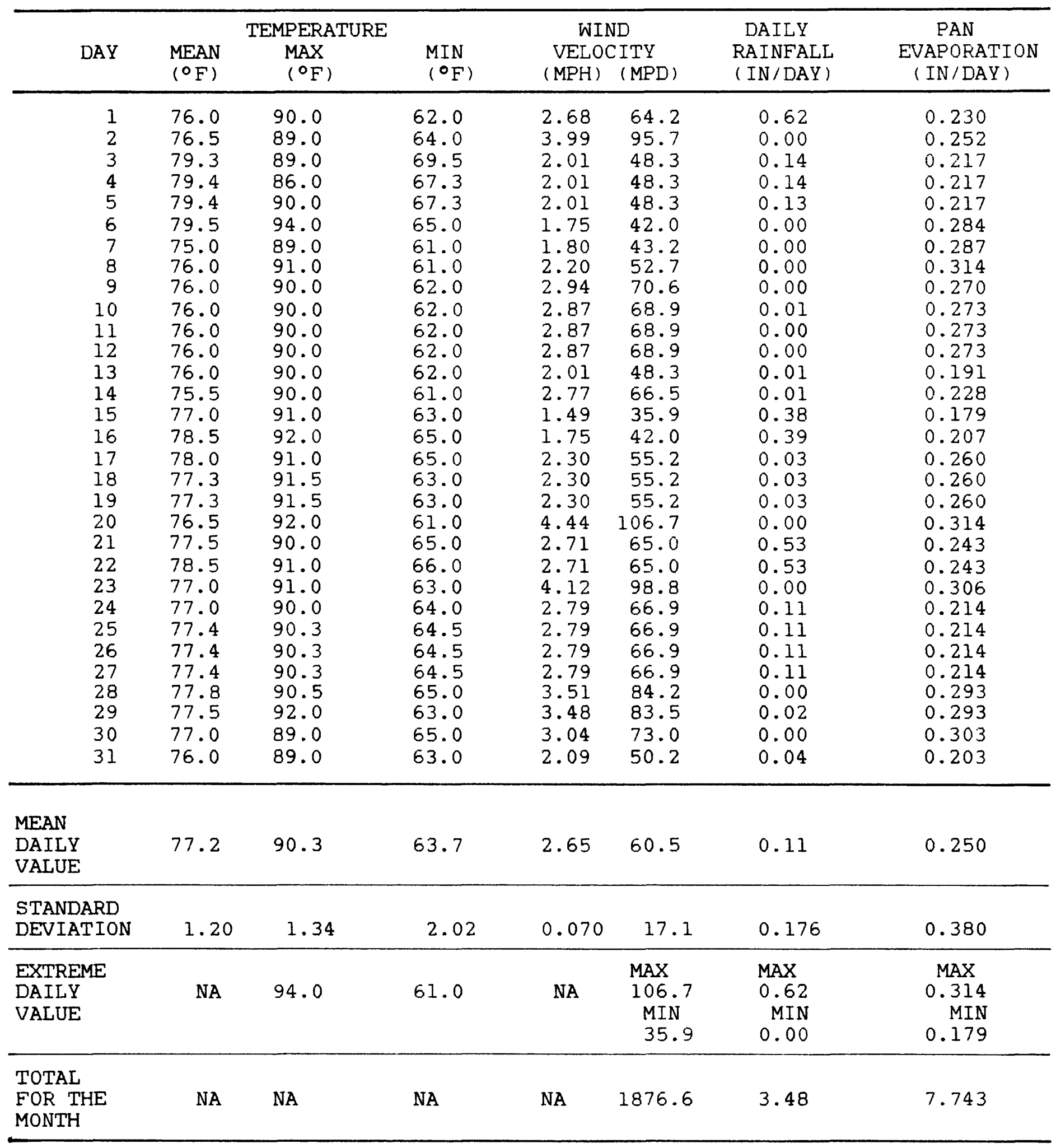


Table 3. (Contlnued)

ARECIBO SEPTEMBER 1984

\begin{tabular}{|c|c|c|c|c|c|c|c|}
\hline DAY & $\begin{array}{l}\text { MEAN } \\
\left(\mathrm{OF}^{\circ}\right)\end{array}$ & $\begin{array}{c}\text { MMPERAI } \\
\text { MAX } \\
\left({ }^{\circ} \mathrm{F}\right)\end{array}$ & $\begin{array}{l}\text { MIN } \\
\left({ }^{\circ} F\right)\end{array}$ & $\begin{array}{l}\text { WIN } \\
\text { VELOC } \\
(\mathrm{MPH})\end{array}$ & $\begin{array}{l}\text { ND } \\
\text { CITY } \\
\text { (MPD) }\end{array}$ & $\begin{array}{l}\text { DAILY } \\
\text { RAINFALL } \\
\text { (IN/DAY) }\end{array}$ & $\begin{array}{c}\text { PAN } \\
\text { EVAPORATION } \\
(\text { IN / DAY })\end{array}$ \\
\hline $\begin{array}{r}1 \\
2 \\
3 \\
4 \\
5 \\
6 \\
7 \\
8 \\
9 \\
10 \\
11 \\
12 \\
13 \\
14 \\
15 \\
16 \\
17 \\
18 \\
19 \\
20 \\
21 \\
22 \\
23 \\
24 \\
25 \\
26 \\
27 \\
28 \\
29 \\
30\end{array}$ & $\begin{array}{l}79.3 \\
79.3 \\
79.3 \\
79.5 \\
79.8 \\
79.8 \\
81.0 \\
80.3 \\
80.3 \\
79.5 \\
79.8 \\
79.8 \\
80.0 \\
79.5 \\
79.8 \\
79.8 \\
80.0 \\
79.3 \\
78.5 \\
78.5 \\
80.3 \\
78.8 \\
78.8 \\
77.3 \\
78.8 \\
78.8 \\
78.8 \\
80.3 \\
78.4 \\
78.4\end{array}$ & $\begin{array}{l}89.5 \\
89.5 \\
89.5 \\
90.0 \\
91.0 \\
91.0 \\
92.0 \\
91.0 \\
91.0 \\
90.0 \\
90.0 \\
90.0 \\
90.0 \\
88.0 \\
89.0 \\
89.0 \\
90.0 \\
89.0 \\
88.0 \\
87.0 \\
89.5 \\
89.5 \\
89.5 \\
89.5 \\
91.5 \\
90.5 \\
90.5 \\
88.5 \\
81.3 \\
81.3\end{array}$ & $\begin{array}{l}69.0 \\
69.0 \\
69.0 \\
69.0 \\
69.5 \\
69.5 \\
70.0 \\
69.5 \\
69.5 \\
69.0 \\
67.0 \\
68.5 \\
70.0 \\
71.0 \\
70.5 \\
70.5 \\
70.0 \\
69.5 \\
69.0 \\
70.0 \\
71.0 \\
68.0 \\
68.0 \\
65.0 \\
69.0 \\
68.5 \\
68.5 \\
68.0 \\
65.0 \\
65.0\end{array}$ & $\begin{array}{l}2.09 \\
2.09 \\
2.09 \\
2.21 \\
2.21 \\
2.21 \\
2.88 \\
2.88 \\
2.88 \\
2.31 \\
1.30 \\
1.30 \\
1.33 \\
1.70 \\
1.70 \\
1.70 \\
0.69 \\
0.69 \\
0.80 \\
1.36 \\
0.86 \\
0.86 \\
0.86 \\
0.74 \\
2.03 \\
2.03 \\
2.03 \\
0.92 \\
0.92 \\
0.92\end{array}$ & $\begin{array}{l}50.2 \\
50.2 \\
50.2 \\
53.1 \\
53.1 \\
53.1 \\
69.1 \\
69.1 \\
69.1 \\
55.4 \\
31.2 \\
31.2 \\
32.0 \\
40.9 \\
40.9 \\
40.9 \\
16.6 \\
16.6 \\
19.2 \\
32.6 \\
20.7 \\
20.7 \\
20.7 \\
17.7 \\
48.7 \\
48.7 \\
48.7 \\
22.2 \\
22.2 \\
22.2\end{array}$ & $\begin{array}{l}0.03 \\
0.03 \\
0.03 \\
0.23 \\
0.22 \\
0.22 \\
0.00 \\
0.00 \\
0.00 \\
0.01 \\
0.66 \\
0.65 \\
0.11 \\
0.20 \\
0.19 \\
0.19 \\
1.37 \\
1.36 \\
0.21 \\
0.00 \\
0.20 \\
0.20 \\
0.10 \\
0.00 \\
0.00 \\
0.00 \\
0.00 \\
0.01 \\
0.00 \\
0.00\end{array}$ & $\begin{array}{l}0.203 \\
0.203 \\
0.203 \\
0.203 \\
0.203 \\
0.203 \\
0.283 \\
0.283 \\
0.283 \\
0.145 \\
0.184 \\
0.184 \\
0.103 \\
0.069 \\
0.069 \\
0.069 \\
1.430 \\
1.430 \\
0.163 \\
0.153 \\
0.154 \\
0.154 \\
0.154 \\
0.119 \\
0.223 \\
0.223 \\
0.223 \\
0.243 \\
0.243 \\
0.243\end{array}$ \\
\hline $\begin{array}{l}\text { MEAN } \\
\text { DAILY } \\
\text { VALUE }\end{array}$ & 79.4 & 89.4 & 68.9 & 1.62 & 38.9 & 0.19 & 0.181 \\
\hline $\begin{array}{l}\text { STANDARD } \\
\text { DEVIATION }\end{array}$ & 0.761 & 2.37 & 1.56 & 0.688 & 16.5 & 0.352 & 0.328 \\
\hline $\begin{array}{l}\text { EXTREME } \\
\text { DAILY } \\
\text { VALUE }\end{array}$ & NA & 92.0 & 65.0 & $\mathrm{NA}$ & $\begin{array}{l}\operatorname{MAX} \\
69.1 \\
\text { MIN } \\
16.6\end{array}$ & $\begin{array}{l}\text { MAX } \\
1.37 \\
\text { MIN } \\
0.00\end{array}$ & $\begin{array}{c}\text { MAX } \\
1.43 \\
\text { MIN } \\
0.069\end{array}$ \\
\hline $\begin{array}{l}\text { TOTAL } \\
\text { FOR THE } \\
\text { MONTH }\end{array}$ & NA & $\mathrm{NA}$ & NA & $\mathrm{NA}$ & 1167.0 & 5.76 & 5.417 \\
\hline
\end{tabular}




\section{Table 3. (Continued) \\ ARECIBO OCTOBER 1984}

\begin{tabular}{|c|c|c|c|c|c|c|c|}
\hline DAY & $\begin{array}{l}\text { MEAN } \\
\left(O^{\circ} \mathrm{F}\right)\end{array}$ & $\begin{array}{c}\text { TEMFERATURE } \\
\text { MAX } \\
(\circ \mathrm{F})\end{array}$ & $\begin{array}{l}\text { MIN } \\
(O F)\end{array}$ & $\begin{array}{r}\text { WI } \\
\text { VELO } \\
(M P H)\end{array}$ & $\begin{array}{l}\text { JD } \\
\text { (MPD) }\end{array}$ & $\begin{array}{c}\text { DAILY } \\
\text { RAINFALL } \\
(\text { IN/DAY })\end{array}$ & $\begin{array}{c}\text { PAN } \\
\text { EVAPORATION } \\
\text { (IN/DAY) }\end{array}$ \\
\hline $\begin{array}{l}1 \\
2 \\
3 \\
4 \\
5 \\
6 \\
7 \\
8 \\
9 \\
10 \\
11 \\
12 \\
13 \\
14 \\
15 \\
16 \\
17 \\
18 \\
19 \\
20 \\
21 \\
22 \\
23 \\
24 \\
25 \\
26 \\
27 \\
28 \\
29 \\
30 \\
31\end{array}$ & $\begin{array}{l}78.4 \\
75.5 \\
76.5 \\
76.3 \\
76.3 \\
76.3 \\
76.3 \\
76.3 \\
76.0 \\
75.0 \\
74.5 \\
75.0 \\
75.0 \\
75.0 \\
75.5 \\
74.5 \\
74.5 \\
74.5 \\
73.5 \\
74.0 \\
74.0 \\
74.5 \\
74.0 \\
72.5 \\
75.0 \\
76.5 \\
75.8 \\
75.8 \\
75.0 \\
74.5 \\
74.0\end{array}$ & $\begin{array}{l}89.3 \\
88.0 \\
90.0 \\
89.0 \\
89.0 \\
89.0 \\
89.0 \\
89.0 \\
88.0 \\
86.0 \\
87.0 \\
88.0 \\
88.0 \\
88.0 \\
89.0 \\
85.0 \\
87.0 \\
87.0 \\
86.0 \\
86.5 \\
86.5 \\
87.0 \\
85.0 \\
84.0 \\
88.0 \\
89.0 \\
87.5 \\
87.5 \\
86.0 \\
85.5 \\
85.0\end{array}$ & $\begin{array}{l}65.5 \\
63.0 \\
63.0 \\
63.5 \\
63.5 \\
63.5 \\
63.5 \\
63.5 \\
64.0 \\
64.0 \\
62.0 \\
62.0 \\
62.0 \\
62.0 \\
62.0 \\
63.0 \\
62.0 \\
62.0 \\
61.0 \\
61.5 \\
61.5 \\
62.0 \\
63.0 \\
61.0 \\
62.0 \\
64.0 \\
64.0 \\
64.0 \\
64.0 \\
63.5 \\
63.0\end{array}$ & $\begin{array}{l}0.92 \\
0.86 \\
0.70 \\
0.70 \\
0.70 \\
0.70 \\
0.70 \\
0.70 \\
0.26 \\
1.09 \\
1.09 \\
1.09 \\
1.09 \\
1.09 \\
1.09 \\
0.50 \\
1.35 \\
1.35 \\
1.35 \\
1.35 \\
1.35 \\
1.70 \\
0.78 \\
0.78 \\
0.78 \\
1.73 \\
1.73 \\
1.73 \\
0.72 \\
0.72 \\
1.47\end{array}$ & $\begin{array}{l}22.2 \\
20.6 \\
16.7 \\
16.7 \\
16.7 \\
16.7 \\
16.7 \\
16.7 \\
6.3 \\
26.3 \\
26.3 \\
26.3 \\
26.3 \\
26.3 \\
26.3 \\
12.0 \\
32.4 \\
32.4 \\
32.4 \\
32.4 \\
32.4 \\
40.9 \\
18.7 \\
18.7 \\
18.7 \\
41.6 \\
41.6 \\
41.6 \\
17.2 \\
17.2 \\
35.4\end{array}$ & $\begin{array}{l}0.00 \\
0.57 \\
0.49 \\
0.49 \\
0.48 \\
0.48 \\
0.48 \\
0.48 \\
0.02 \\
0.09 \\
0.09 \\
0.08 \\
0.08 \\
0.08 \\
0.08 \\
0.01 \\
0.59 \\
0.59 \\
0.59 \\
0.59 \\
0.59 \\
0.08 \\
0.00 \\
0.00 \\
0.00 \\
0.50 \\
0.49 \\
0.49 \\
0.03 \\
0.03 \\
0.02\end{array}$ & $\begin{array}{l}0.243 \\
0.248 \\
0.456 \\
0.456 \\
0.456 \\
0.456 \\
0.456 \\
0.456 \\
0.149 \\
0.157 \\
0.157 \\
0.157 \\
0.157 \\
0.157 \\
0.157 \\
0.663 \\
0.133 \\
0.133 \\
0.133 \\
0.133 \\
0.133 \\
0.169 \\
0.141 \\
0.141 \\
0.141 \\
0.179 \\
0.179 \\
0.179 \\
0.201 \\
0.201 \\
0.052\end{array}$ \\
\hline $\begin{array}{l}\text { MEAN } \\
\text { DAILY } \\
\text { VALUE }\end{array}$ & 75.2 & 87.4 & 62.8 & 0.95 & 27.9 & 0.28 & 0.233 \\
\hline $\begin{array}{l}\text { STANDARD } \\
\text { DEVIATION }\end{array}$ & 1.14 & 1.47 & 1.06 & 0.395 & 9.75 & 0.246 & 0.145 \\
\hline $\begin{array}{l}\text { EXTREME } \\
\text { DAILY } \\
\text { VALUE }\end{array}$ & $\mathrm{NA}$ & 90.0 & 61.0 & NA & $\begin{array}{l}\text { MAX } \\
40.9 \\
\text { MIN } \\
6.3\end{array}$ & $\begin{array}{l}\text { MAX } \\
0.59 \\
\text { MIN } \\
0.00\end{array}$ & $\begin{array}{c}\text { MAX } \\
0.663 \\
\text { MIN } \\
0.052\end{array}$ \\
\hline $\begin{array}{l}\text { TOTAL } \\
\text { FOR THE } \\
\text { MONTH }\end{array}$ & NA & $\mathrm{NA}$ & NA & NA & 864.9 & 8.59 & 7.223 \\
\hline
\end{tabular}


Table 3. (Continued)

ARECIBO NOVEMBER 1984

\begin{tabular}{|c|c|c|c|c|c|c|c|}
\hline DAY & $\begin{array}{l}\text { MEAN } \\
(O F)\end{array}$ & $\begin{array}{c}\text { MEMPERA' } \\
\text { MAX } \\
(O F)\end{array}$ & $\begin{array}{l}\text { MIN } \\
(\circ \mathrm{F})\end{array}$ & \multicolumn{2}{|c|}{$\begin{array}{c}\text { WIND } \\
\text { VELOCITY }\end{array}$} & $\begin{array}{c}\text { DAILY } \\
\text { RAINFALL } \\
\text { (IN/DAY) }\end{array}$ & $\begin{array}{c}\text { PAN } \\
\text { EVAPORATION } \\
\text { (IN/DAY) }\end{array}$ \\
\hline $\begin{array}{l}1 \\
2 \\
3 \\
4 \\
5 \\
6 \\
7 \\
8 \\
9 \\
10 \\
11 \\
12 \\
13 \\
14 \\
15 \\
16 \\
17 \\
18 \\
19 \\
20 \\
21 \\
22 \\
23 \\
24 \\
25 \\
26 \\
27 \\
28 \\
29 \\
30\end{array}$ & $\begin{array}{l}73.0 \\
74.0 \\
74.3 \\
74.3 \\
74.5 \\
75.0 \\
75.0 \\
75.5 \\
75.0 \\
75.3 \\
75.3 \\
75.3 \\
75.5 \\
74.8 \\
74.0 \\
74.5 \\
74.7 \\
74.7 \\
74.8 \\
73.5 \\
75.0 \\
74.5 \\
74.0 \\
75.3 \\
75.3 \\
76.5 \\
76.5 \\
73.5 \\
72.5 \\
72.5\end{array}$ & $\begin{array}{l}85.0 \\
85.0 \\
85.5 \\
85.5 \\
86.0 \\
85.5 \\
85.5 \\
85.0 \\
86.0 \\
85.5 \\
85.5 \\
85.5 \\
85.0 \\
84.0 \\
83.0 \\
85.0 \\
85.3 \\
85.3 \\
85.5 \\
84.0 \\
85.0 \\
84.5 \\
84.0 \\
87.0 \\
87.0 \\
90.0 \\
85.0 \\
82.0 \\
82.5 \\
82.0\end{array}$ & $\begin{array}{l}61.0 \\
63.0 \\
63.0 \\
63.0 \\
63.0 \\
64.5 \\
64.5 \\
66.0 \\
68.0 \\
67.0 \\
67.0 \\
67.0 \\
66.0 \\
65.0 \\
65.0 \\
64.0 \\
64.0 \\
64.0 \\
64.0 \\
63.0 \\
65.0 \\
64.5 \\
64.0 \\
63.5 \\
63.5 \\
63.0 \\
68.0 \\
65.0 \\
63.0 \\
63.0\end{array}$ & $\begin{array}{l}1.17 \\
0.48 \\
0.48 \\
0.48 \\
1.31 \\
1.31 \\
1.31 \\
2.86 \\
1.35 \\
1.35 \\
1.35 \\
1.35 \\
1.34 \\
1.34 \\
1.34 \\
1.22 \\
1.22 \\
1.22 \\
1.69 \\
1.69 \\
1.27 \\
1.27 \\
0.25 \\
0.25 \\
0.25 \\
1.26 \\
0.76 \\
0.70 \\
1.09 \\
1.44\end{array}$ & $\begin{array}{r}28.0 \\
11.6 \\
11.6 \\
11.6 \\
31.5 \\
31.5 \\
31.5 \\
68.7 \\
32.5 \\
32.5 \\
32.5 \\
32.5 \\
32.3 \\
32.3 \\
32.3 \\
29.2 \\
29.2 \\
29.2 \\
40.6 \\
40.6 \\
30.6 \\
30.6 \\
6.0 \\
6.0 \\
6.0 \\
30.4 \\
18.4 \\
16.7 \\
24.2 \\
34.5\end{array}$ & $\begin{array}{l}0.01 \\
0.52 \\
0.51 \\
0.51 \\
0.41 \\
0.41 \\
0.40 \\
0.21 \\
0.31 \\
0.31 \\
0.30 \\
0.30 \\
0.41 \\
0.40 \\
0.01 \\
0.19 \\
0.18 \\
0.18 \\
0.01 \\
0.00 \\
0.01 \\
0.01 \\
0.02 \\
0.01 \\
0.01 \\
0.18 \\
0.14 \\
0.00 \\
0.07 \\
0.17\end{array}$ & $\begin{array}{l}0.121 \\
0.060 \\
0.060 \\
0.060 \\
0.065 \\
0.065 \\
0.065 \\
0.249 \\
0.397 \\
0.397 \\
0.397 \\
0.397 \\
0.049 \\
0.049 \\
0.325 \\
0.126 \\
0.126 \\
0.126 \\
0.259 \\
0.259 \\
0.128 \\
0.128 \\
0.103 \\
0.103 \\
0.103 \\
0.082 \\
0.167 \\
0.063 \\
0.181 \\
0.138\end{array}$ \\
\hline $\begin{array}{l}\text { MEAN } \\
\text { DAILY } \\
\text { VALUE }\end{array}$ & 74.6 & 85.0 & 64.5 & 1.15 & 27.5 & 0.19 & 0.162 \\
\hline $\begin{array}{l}\text { STANDARD } \\
\text { DEVIATION }\end{array}$ & 0.967 & 1.51 & 1.66 & 0.520 & 12.5 & 0.178 & 0.115 \\
\hline $\begin{array}{l}\text { EXTREME } \\
\text { DAILY } \\
\text { VALUE }\end{array}$ & NA & 90.0 & 61.0 & NA & $\begin{array}{l}\operatorname{MAX} \\
68.7 \\
\text { MIN } \\
6.0\end{array}$ & $\begin{array}{l}\text { MAX } \\
0.52 \\
\text { MIN } \\
0.00\end{array}$ & $\begin{array}{c}\text { MAX } \\
0.397 \\
\text { MIN } \\
0.049\end{array}$ \\
\hline $\begin{array}{l}\text { TOTAL } \\
\text { FOR THE } \\
\text { MONTH }\end{array}$ & $\mathrm{NA}$ & $\mathrm{NA}$ & NA & NA & 825.0 & 5.84 & 4.848 \\
\hline
\end{tabular}


Table 3. (Continued)

ARECIBO DECEMBER 1984

\begin{tabular}{|c|c|c|c|c|c|c|c|}
\hline DAY & $\begin{array}{l}\text { MEAN } \\
(\text { OF })\end{array}$ & $\begin{array}{l}\text { TEMPERATURE } \\
\text { MAX } \\
(\circ \mathrm{F})\end{array}$ & $\begin{array}{l}\text { MIN } \\
(\text { OF) }\end{array}$ & $\begin{array}{r}\text { WI } \\
\text { VELO } \\
\text { (MPH) }\end{array}$ & $\begin{array}{l}\text { ND } \\
\text { CITY } \\
\text { (MPD) }\end{array}$ & $\begin{array}{c}\text { DAILY } \\
\text { RAINFALL } \\
\text { (IN/DAY) }\end{array}$ & $\begin{array}{c}\text { PAN } \\
\text { EVAPORATION } \\
\text { (IN / DAY) }\end{array}$ \\
\hline $\begin{array}{l}1 \\
2 \\
3 \\
4 \\
5 \\
6 \\
7 \\
8 \\
9 \\
10 \\
11 \\
12 \\
13 \\
14 \\
15 \\
16 \\
17 \\
18 \\
19 \\
20 \\
21 \\
22 \\
23 \\
24 \\
25 \\
26 \\
27 \\
28 \\
29 \\
30 \\
31\end{array}$ & $\begin{array}{l}73.0 \\
73.0 \\
73.5 \\
73.0 \\
74.5 \\
75.5 \\
74.5 \\
74.5 \\
74.5 \\
73.5 \\
74.0 \\
74.5 \\
73.0 \\
72.5 \\
73.3 \\
73.3 \\
74.0 \\
71.5 \\
70.5 \\
69.5 \\
70.0 \\
70.5 \\
70.5 \\
70.5 \\
70.5 \\
71.0 \\
71.9 \\
71.9 \\
71.9 \\
71.9 \\
72.8\end{array}$ & $\begin{array}{l}83.0 \\
83.0 \\
84.0 \\
84.0 \\
84.0 \\
84.0 \\
83.5 \\
83.5 \\
83.5 \\
83.0 \\
83.0 \\
84.0 \\
83.0 \\
82.0 \\
82.0 \\
82.0 \\
82.0 \\
81.0 \\
79.5 \\
78.0 \\
81.0 \\
82.0 \\
82.0 \\
82.0 \\
82.0 \\
83.0 \\
82.8 \\
82.8 \\
82.8 \\
82.8 \\
82.5\end{array}$ & $\begin{array}{l}63.0 \\
63.0 \\
63.0 \\
64.0 \\
65.0 \\
67.0 \\
65.5 \\
65.5 \\
65.5 \\
64.0 \\
65.0 \\
65.0 \\
63.0 \\
63.0 \\
64.5 \\
64.5 \\
66.0 \\
62.0 \\
61.5 \\
61.0 \\
59.0 \\
59.0 \\
59.0 \\
59.0 \\
59.0 \\
59.0 \\
61.0 \\
61.0 \\
61.0 \\
61.0 \\
63.0\end{array}$ & $\begin{array}{l}1.44 \\
1.44 \\
1.99 \\
1.99 \\
1.99 \\
1.28 \\
1.28 \\
1.28 \\
1.28 \\
1.28 \\
0.04 \\
0.39 \\
2.84 \\
2.84 \\
2.84 \\
2.84 \\
2.84 \\
1.82 \\
1.82 \\
1.82 \\
1.82 \\
1.82 \\
1.82 \\
1.82 \\
1.82 \\
1.46 \\
1.46 \\
1.46 \\
1.46 \\
1.46 \\
0.24\end{array}$ & $\begin{array}{r}34.5 \\
34.5 \\
47.7 \\
47.7 \\
47.7 \\
30.6 \\
30.6 \\
30.6 \\
30.6 \\
30.6 \\
10.0 \\
9.5 \\
68.1 \\
68.1 \\
68.1 \\
68.1 \\
68.1 \\
43.7 \\
43.7 \\
43.7 \\
43.7 \\
43.7 \\
43.7 \\
43.7 \\
43.7 \\
35.0 \\
35.0 \\
35.0 \\
35.0 \\
35.0 \\
5.8\end{array}$ & $\begin{array}{l}0.17 \\
0.16 \\
0.11 \\
0.11 \\
0.11 \\
0.35 \\
0.35 \\
0.35 \\
0.35 \\
0.35 \\
0.02 \\
0.01 \\
0.14 \\
0.14 \\
0.13 \\
0.13 \\
0.13 \\
0.22 \\
0.22 \\
0.22 \\
0.22 \\
0.22 \\
0.22 \\
0.21 \\
0.21 \\
0.49 \\
0.49 \\
0.48 \\
0.48 \\
0.48 \\
0.33\end{array}$ & $\begin{array}{l}0.138 \\
0.138 \\
0.174 \\
0.174 \\
0.174 \\
0.149 \\
0.149 \\
0.149 \\
0.149 \\
0.149 \\
0.189 \\
0.107 \\
0.183 \\
0.183 \\
0.183 \\
0.183 \\
0.183 \\
0.126 \\
0.126 \\
0.126 \\
0.126 \\
0.126 \\
0.126 \\
0.126 \\
0.126 \\
0.106 \\
0.106 \\
0.106 \\
0.106 \\
0.106 \\
0.122\end{array}$ \\
\hline $\begin{array}{l}\text { MEAN } \\
\text { DAILY } \\
\text { VALUE }\end{array}$ & 72.5 & 82.5 & 62.6 & 1.68 & 40.5 & 0.25 & 0.142 \\
\hline $\begin{array}{l}\text { STANDARD } \\
\text { DEVIATION }\end{array}$ & 1.58 & 1.29 & 2.39 & 0.690 & 15.9 & 0.138 & 0.028 \\
\hline $\begin{array}{l}\text { EXTREME } \\
\text { DAILY } \\
\text { VALUE }\end{array}$ & NA & 84.0 & 59.0 & NA & $\begin{array}{l}\text { MAX } \\
68.1 \\
\text { MIN } \\
5.8\end{array}$ & $\begin{array}{l}\text { MAX } \\
0.49 \\
\text { MIN } \\
0.01\end{array}$ & $\begin{array}{c}\text { MAX } \\
0.189 \\
\text { MIN } \\
0.106\end{array}$ \\
\hline $\begin{array}{l}\text { TOTAL } \\
\text { FOR THE } \\
\text { MONTH }\end{array}$ & NA & NA & NA & NA & 1255.5 & 7.60 & 4.414 \\
\hline
\end{tabular}


Table 3. (Continued)

ARECIBO JANUARY 1985

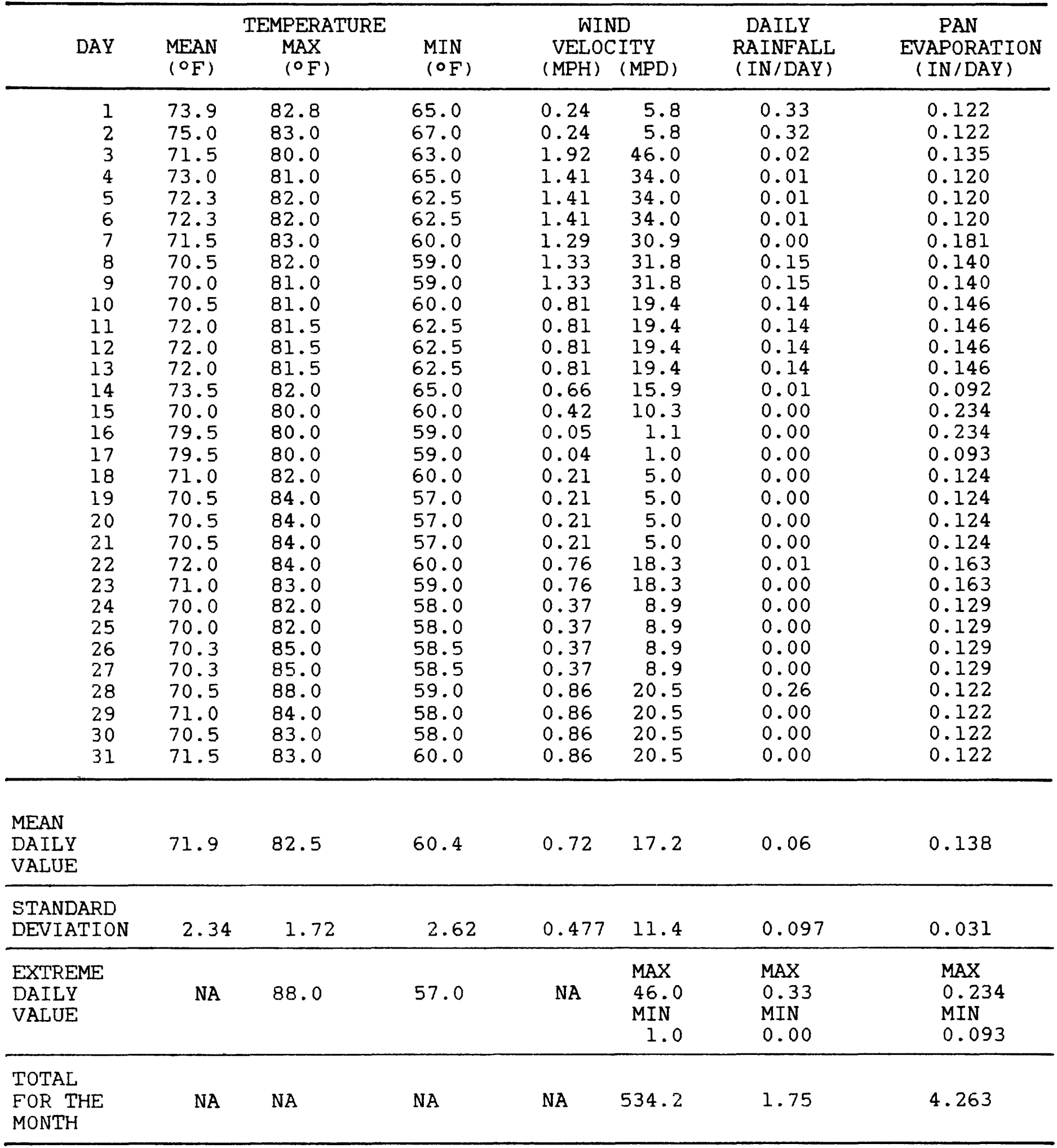


Table 3. (Contlnued)

ARECIBO FEBRUARY 1985

\begin{tabular}{cccccccc}
\hline DAY & MEAN & $\begin{array}{c}\text { TEMPERATURE } \\
\text { MAX } \\
(\text { OF })\end{array}$ & $\begin{array}{c}\text { MIN } \\
\text { (OF) }\end{array}$ & $\begin{array}{c}\text { WIND } \\
\text { VELOCITY } \\
(\text { MPH) }\end{array}$ & $\begin{array}{c}\text { DAILY } \\
\text { RAINFALL } \\
\text { (IN/DAY) }\end{array}$ & $\begin{array}{c}\text { PAN } \\
\text { EVAPORATION } \\
\text { (IN/DAY) }\end{array}$ \\
\hline 1 & 71.0 & 81.0 & 61.0 & 0.97 & 23.2 & 0.01 & 0.143 \\
2 & 73.5 & 82.0 & 63.0 & 0.97 & 23.2 & 0.01 & 0.143 \\
3 & 73.5 & 82.0 & 53.0 & 0.97 & 23.2 & 0.01 & 0.143 \\
4 & 73.5 & 83.0 & 65.0 & 0.97 & 23.2 & 0.01 & 0.143 \\
5 & 73.5 & 83.0 & 63.0 & 0.97 & 23.2 & 0.01 & 0.143 \\
6 & 73.5 & 82.0 & 57.0 & 0.97 & 23.2 & 0.01 & 0.143 \\
7 & 73.5 & 86.0 & 57.0 & 0.97 & 23.2 & 0.01 & 0.143 \\
8 & 76.0 & 85.0 & 57.0 & 1.07 & 25.5 & 0.06 & 0.140 \\
9 & 73.3 & 85.0 & 61.0 & 1.07 & 25.5 & 0.06 & 0.140 \\
10 & 73.3 & 85.0 & 61.0 & 1.07 & 25.5 & 0.06 & 0.140 \\
11 & 73.3 & 85.0 & 61.0 & 1.07 & 25.5 & 0.06 & 0.140 \\
12 & 73.3 & 85.0 & 65.0 & 1.07 & 25.5 & 0.05 & 0.140 \\
13 & 73.3 & 82.0 & 61.0 & 1.07 & 25.5 & 0.05 & 0.140 \\
14 & 73.3 & 83.0 & 58.0 & 1.16 & 27.8 & 0.04 & 0.134 \\
15 & 70.5 & 82.0 & 58.0 & 1.16 & 27.8 & 0.04 & 0.134 \\
16 & 70.8 & 85.5 & 58.5 & 1.16 & 27.8 & 0.04 & 0.134 \\
17 & 70.8 & 85.5 & 58.5 & 1.16 & 27.8 & 0.04 & 0.134 \\
18 & 70.8 & 85.5 & 58.5 & 1.16 & 27.8 & 0.03 & 0.134 \\
19 & 70.8 & 89.0 & 59.0 & 1.16 & 27.8 & 0.03 & 0.134 \\
20 & 71.0 & 83.0 & 59.0 & 1.63 & 39.0 & 0.01 & 0.175 \\
21 & 73.0 & 86.0 & 60.0 & 1.56 & 37.4 & 0.26 & 0.120 \\
22 & 72.5 & 86.0 & 60.0 & 1.56 & 37.4 & 0.26 & 0.120 \\
23 & 72.5 & 84.5 & 60.5 & 1.56 & 37.4 & 0.26 & 0.120 \\
24 & 72.5 & 84.5 & 60.5 & 1.56 & 37.4 & 0.25 & 0.120 \\
25 & 72.5 & 83.0 & 61.0 & 1.56 & 37.4 & 0.25 & 0.120 \\
26 & 72.5 & 82.0 & 60.0 & 1.56 & 37.4 & 0.25 & 0.120 \\
27 & 72.0 & 83.0 & 61.0 & 1.48 & 35.5 & 0.05 & 0.154 \\
28 & 73.0 & 83.0 & 63.0 & 1.48 & 35.5 & 0.04 & 0.154 \\
& & & & & & & \\
\end{tabular}

MEAN

DAILY

VALUE

$72.6 \quad 84.0$

60.4

$1.22 \quad 29.2$

0.08

0.137

STANDARD

DEVIATION

$1.23 \quad 1.79$

2.18

$0.238 \quad 5.75$

0.093

0.012

EXTREME

DAILY

$\mathrm{NA} \quad 89.0$

MAX
NA $\quad 39.0$
MIN

MAX

0.26

MAX

VALUE

23.2

MIN

0.154

MIN

0.120

TOTAL

FOR THE

MONTH

NA NA NA

NA

816.6

2.26

3.848 
Table 3. (Continued)

ARECIBO MARCH 1985

\begin{tabular}{|c|c|c|c|c|c|c|c|}
\hline DAY & $\begin{array}{l}\text { MEAN } \\
\left({ }^{\circ} \mathrm{F}\right)\end{array}$ & $\begin{array}{c}\text { TEMPERATURE } \\
\text { MAX } \\
\left({ }^{\circ} \mathrm{F}\right)\end{array}$ & $\begin{array}{l}\text { MIN } \\
(\circ \mathrm{F})\end{array}$ & $\begin{array}{l}\text { WII } \\
\text { VELOC } \\
\text { (MPH) }\end{array}$ & $\begin{array}{l}\text { ND } \\
\text { CITY } \\
\text { (MPD) }\end{array}$ & $\begin{array}{l}\text { DAILY } \\
\text { RAINFALL } \\
\text { ( IN/DAY) }\end{array}$ & $\begin{array}{c}\text { PAN } \\
\text { EVAPORATION } \\
\text { (IN/DAY) }\end{array}$ \\
\hline $\begin{array}{l}1 \\
2 \\
3 \\
4 \\
5 \\
6 \\
7 \\
8 \\
9 \\
10 \\
11 \\
12 \\
13 \\
14 \\
15 \\
16 \\
17 \\
18 \\
19 \\
20 \\
21 \\
22 \\
23 \\
24 \\
25 \\
26 \\
27 \\
28 \\
29 \\
30 \\
31\end{array}$ & $\begin{array}{l}72.0 \\
72.3 \\
72.3 \\
72.5 \\
72.5 \\
69.5 \\
71.0 \\
73.0 \\
72.5 \\
72.5 \\
72.0 \\
72.5 \\
73.0 \\
72.5 \\
70.5 \\
71.5 \\
71.5 \\
72.5 \\
71.5 \\
73.5 \\
73.5 \\
73.0 \\
73.0 \\
73.0 \\
72.5 \\
73.0 \\
74.5 \\
72.5 \\
73.5 \\
72.5 \\
72.5\end{array}$ & $\begin{array}{l}82.0 \\
83.0 \\
83.0 \\
84.0 \\
83.0 \\
80.0 \\
83.0 \\
82.0 \\
82.5 \\
82.5 \\
83.0 \\
84.0 \\
84.0 \\
84.0 \\
83.0 \\
85.0 \\
85.0 \\
87.0 \\
84.0 \\
86.0 \\
86.0 \\
85.5 \\
85.5 \\
85.5 \\
85.0 \\
84.0 \\
84.0 \\
80.0 \\
82.0 \\
82.0 \\
82.0\end{array}$ & $\begin{array}{l}62.0 \\
61.5 \\
61.5 \\
61.0 \\
62.0 \\
59.0 \\
59.0 \\
64.0 \\
62.5 \\
62.5 \\
61.0 \\
61.0 \\
62.0 \\
61.0 \\
58.0 \\
58.0 \\
58.0 \\
58.0 \\
59.0 \\
61.0 \\
61.0 \\
60.5 \\
60.5 \\
60.5 \\
60.0 \\
62.0 \\
65.0 \\
65.0 \\
65.0 \\
63.0 \\
63.0\end{array}$ & $\begin{array}{l}1.48 \\
1.48 \\
1.48 \\
1.48 \\
1.48 \\
1.33 \\
1.33 \\
1.57 \\
1.57 \\
1.57 \\
1.57 \\
1.64 \\
1.64 \\
1.64 \\
1.64 \\
1.64 \\
1.64 \\
0.58 \\
0.58 \\
0.58 \\
2.22 \\
2.22 \\
2.22 \\
2.22 \\
2.22 \\
4.56 \\
4.56 \\
4.56 \\
2.61 \\
2.61 \\
2.61\end{array}$ & $\begin{array}{r}35.5 \\
35.5 \\
35.5 \\
35.5 \\
35.5 \\
32.0 \\
32.0 \\
37.7 \\
37.7 \\
37.7 \\
37.7 \\
39.5 \\
39.5 \\
39.5 \\
39.5 \\
39.5 \\
39.5 \\
14.1 \\
14.1 \\
14.1 \\
53.1 \\
53.1 \\
53.1 \\
53.1 \\
53.1 \\
109.4 \\
109.4 \\
109.4 \\
62.7 \\
62.7 \\
62.7\end{array}$ & $\begin{array}{l}0.04 \\
0.04 \\
0.04 \\
0.04 \\
0.04 \\
0.51 \\
0.50 \\
0.09 \\
0.09 \\
0.09 \\
0.08 \\
0.01 \\
0.01 \\
0.01 \\
0.01 \\
0.00 \\
0.00 \\
0.96 \\
0.96 \\
0.95 \\
0.00 \\
0.00 \\
0.00 \\
0.00 \\
0.00 \\
0.80 \\
0.79 \\
0.79 \\
0.19 \\
0.19 \\
0.19\end{array}$ & $\begin{array}{l}0.154 \\
0.154 \\
0.154 \\
0.154 \\
0.154 \\
0.094 \\
0.094 \\
0.159 \\
0.159 \\
0.159 \\
0.159 \\
0.188 \\
0.188 \\
0.188 \\
0.188 \\
0.188 \\
0.188 \\
0.070 \\
0.070 \\
0.070 \\
0.215 \\
0.215 \\
0.215 \\
0.215 \\
0.215 \\
0.376 \\
0.376 \\
0.376 \\
0.212 \\
0.212 \\
0.212\end{array}$ \\
\hline $\begin{array}{l}\text { MEAN } \\
\text { DAILY } \\
\text { VALUE }\end{array}$ & 69.5 & 84.0 & 60.6 & 1.95 & 46.1 & 0.24 & 0.240 \\
\hline $\begin{array}{l}\text { STANDARD } \\
\text { DEVIATION }\end{array}$ & 3.05 & 1.70 & 2.08 & 0.996 & 23.9 & 0.337 & 0.093 \\
\hline $\begin{array}{l}\text { EXTREME } \\
\text { DAILY } \\
\text { VALUE }\end{array}$ & $\mathrm{NA}$ & 87.0 & 58.0 & NA & $\begin{array}{l}\text { MAX } \\
109.4 \\
\text { MIN } \\
32.0\end{array}$ & $\begin{array}{l}\text { MAX } \\
0.96 \\
\text { MIN } \\
0.00\end{array}$ & $\begin{array}{c}\text { MAX } \\
0.376 \\
\text { MIN } \\
0.07\end{array}$ \\
\hline $\begin{array}{l}\text { TOTAL } \\
\text { FOR THE } \\
\text { MONTH }\end{array}$ & NA & NA & NA & $\mathrm{NA}$ & 1453.4 & 7.42 & 7.460 \\
\hline
\end{tabular}


Table 3. (Continued)

ARECIBO APRIL 1985

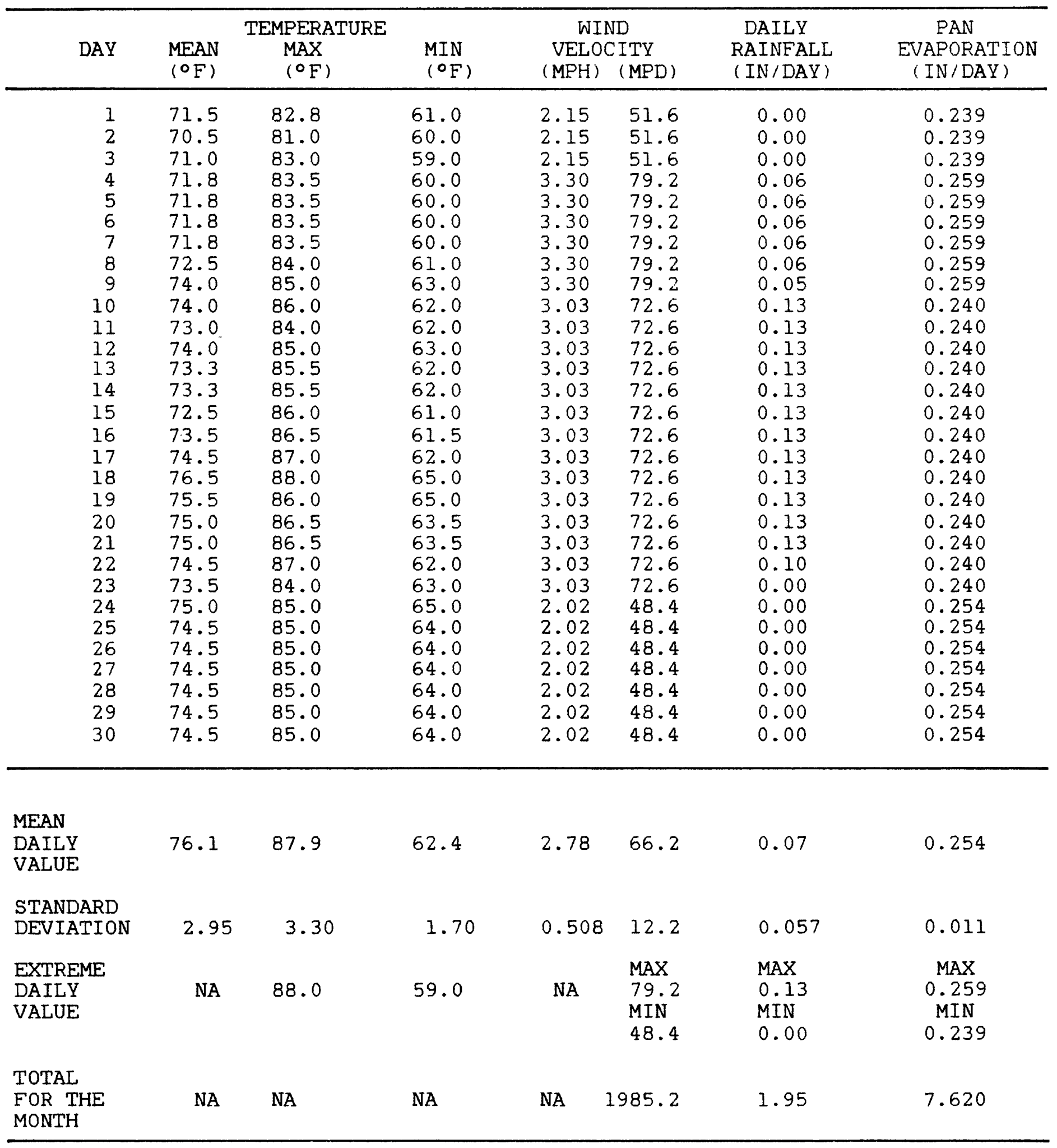


Table 3. (Continued)

ARECIBO MAY 1985

\begin{tabular}{|c|c|c|c|c|c|c|c|}
\hline DAY & $\begin{array}{l}\text { MEAN } \\
(O F)\end{array}$ & $\begin{array}{l}\text { MPPERAT } \\
\text { MAX } \\
\left({ }^{\circ} \mathrm{F}\right)\end{array}$ & $\begin{array}{l}\text { MIN } \\
(O F)\end{array}$ & \multicolumn{2}{|c|}{$\begin{array}{c}\text { WIND } \\
\text { VELOCITY }\end{array}$} & $\begin{array}{c}\text { DAILY } \\
\text { RAINFALL } \\
\text { ( IN/DAY) }\end{array}$ & $\begin{array}{c}\text { PAN } \\
\text { EVAPORATION } \\
\text { (IN/DAY) }\end{array}$ \\
\hline $\begin{array}{l}1 \\
2 \\
3 \\
4 \\
5 \\
6 \\
7 \\
8 \\
9 \\
10 \\
11 \\
12 \\
13 \\
14 \\
15 \\
16 \\
17 \\
18 \\
19 \\
20 \\
21 \\
22 \\
23 \\
24 \\
25 \\
26 \\
27 \\
28 \\
29 \\
30 \\
31\end{array}$ & $\begin{array}{l}74.0 \\
75.0 \\
75.5 \\
75.0 \\
75.0 \\
76.5 \\
76.0 \\
75.0 \\
75.5 \\
75.0 \\
74.3 \\
74.3 \\
73.5 \\
76.5 \\
76.5 \\
72.5 \\
77.5 \\
77.3 \\
77.3 \\
77.0 \\
77.0 \\
75.0 \\
75.0 \\
76.0 \\
78.3 \\
78.3 \\
78.3 \\
80.5 \\
81.0 \\
81.0 \\
80.3\end{array}$ & $\begin{array}{l}85.0 \\
86.0 \\
87.0 \\
88.0 \\
88.0 \\
89.0 \\
88.0 \\
85.0 \\
87.0 \\
86.0 \\
86.0 \\
86.0 \\
86.0 \\
87.0 \\
89.0 \\
80.0 \\
88.0 \\
88.0 \\
88.0 \\
88.0 \\
87.0 \\
86.0 \\
87.0 \\
87.0 \\
91.0 \\
91.0 \\
91.0 \\
95.5 \\
95.0 \\
95.0 \\
93.0\end{array}$ & $\begin{array}{l}63.0 \\
64.0 \\
64.0 \\
64.0 \\
64.0 \\
64.0 \\
64.0 \\
65.0 \\
64.0 \\
64.0 \\
62.5 \\
62.5 \\
61.0 \\
66.0 \\
64.0 \\
65.0 \\
67.0 \\
66.5 \\
66.5 \\
66.0 \\
67.0 \\
64.0 \\
63.0 \\
65.0 \\
65.5 \\
65.5 \\
65.5 \\
66.0 \\
67.0 \\
67.0 \\
66.5\end{array}$ & $\begin{array}{l}2.02 \\
2.23 \\
2.23 \\
2.23 \\
2.23 \\
2.23 \\
2.81 \\
2.81 \\
2.81 \\
3.49 \\
3.49 \\
3.49 \\
3.49 \\
1.89 \\
1.89 \\
1.89 \\
1.90 \\
1.90 \\
1.90 \\
3.47 \\
3.47 \\
3.47 \\
3.47 \\
1.82 \\
1.82 \\
1.82 \\
1.82 \\
1.82 \\
1.82 \\
3.60 \\
3.60\end{array}$ & $\begin{array}{l}48.4 \\
53.4 \\
53.4 \\
53.4 \\
53.4 \\
53.4 \\
67.4 \\
67.4 \\
67.4 \\
83.8 \\
83.8 \\
83.8 \\
83.8 \\
45.4 \\
45.4 \\
45.4 \\
45.6 \\
45.6 \\
45.6 \\
83.4 \\
83.4 \\
83.4 \\
83.4 \\
43.8 \\
43.8 \\
43.8 \\
43.8 \\
43.8 \\
43.8 \\
86.4 \\
86.4\end{array}$ & $\begin{array}{l}0.00 \\
0.04 \\
0.04 \\
0.04 \\
0.04 \\
0.04 \\
0.02 \\
0.02 \\
0.01 \\
0.03 \\
0.03 \\
0.02 \\
0.02 \\
0.10 \\
0.10 \\
0.10 \\
0.40 \\
0.40 \\
0.40 \\
0.00 \\
0.00 \\
0.00 \\
0.00 \\
0.00 \\
0.00 \\
0.00 \\
0.00 \\
0.00 \\
0.00 \\
0.00 \\
0.00\end{array}$ & $\begin{array}{l}0.254 \\
0.213 \\
0.213 \\
0.213 \\
0.213 \\
0.213 \\
0.231 \\
0.231 \\
0.231 \\
0.326 \\
0.326 \\
0.326 \\
0.326 \\
0.162 \\
0.162 \\
0.162 \\
0.134 \\
0.134 \\
0.134 \\
0.406 \\
0.406 \\
0.406 \\
0.406 \\
0.286 \\
0.286 \\
0.286 \\
0.286 \\
0.286 \\
0.286 \\
0.355 \\
0.355\end{array}$ \\
\hline $\begin{array}{l}\text { MEAN } \\
\text { DAILY } \\
\text { VALUE }\end{array}$ & 79.0 & 91.1 & 67.0 & 2.63 & 61.1 & 0.06 & 0.275 \\
\hline $\begin{array}{l}\text { STANDARD } \\
\text { DEVIATION }\end{array}$ & 3.33 & 4.34 & 2.67 & 0.721 & 17.2 & 0.115 & 0.083 \\
\hline $\begin{array}{l}\text { EXTREME } \\
\text { DAILY } \\
\text { VALUE }\end{array}$ & $\mathrm{NA}$ & 95.5 & 61.0 & NA & $\begin{array}{l}\text { MAX } \\
86.4 \\
\text { MIN } \\
43.8\end{array}$ & $\begin{array}{l}\text { MAX } \\
0.40 \\
\text { MIN } \\
0.00\end{array}$ & $\begin{array}{c}\text { MAX } \\
0.406 \\
\text { MIN } \\
0.134\end{array}$ \\
\hline $\begin{array}{l}\text { TOTAL } \\
\text { FOR THE } \\
\text { MONTH }\end{array}$ & NA & $\mathrm{NA}$ & NA & NA & 1895.0 & 1.85 & 8.525 \\
\hline
\end{tabular}


Table 3. (Continued)

ARECIBO JUNE 1985

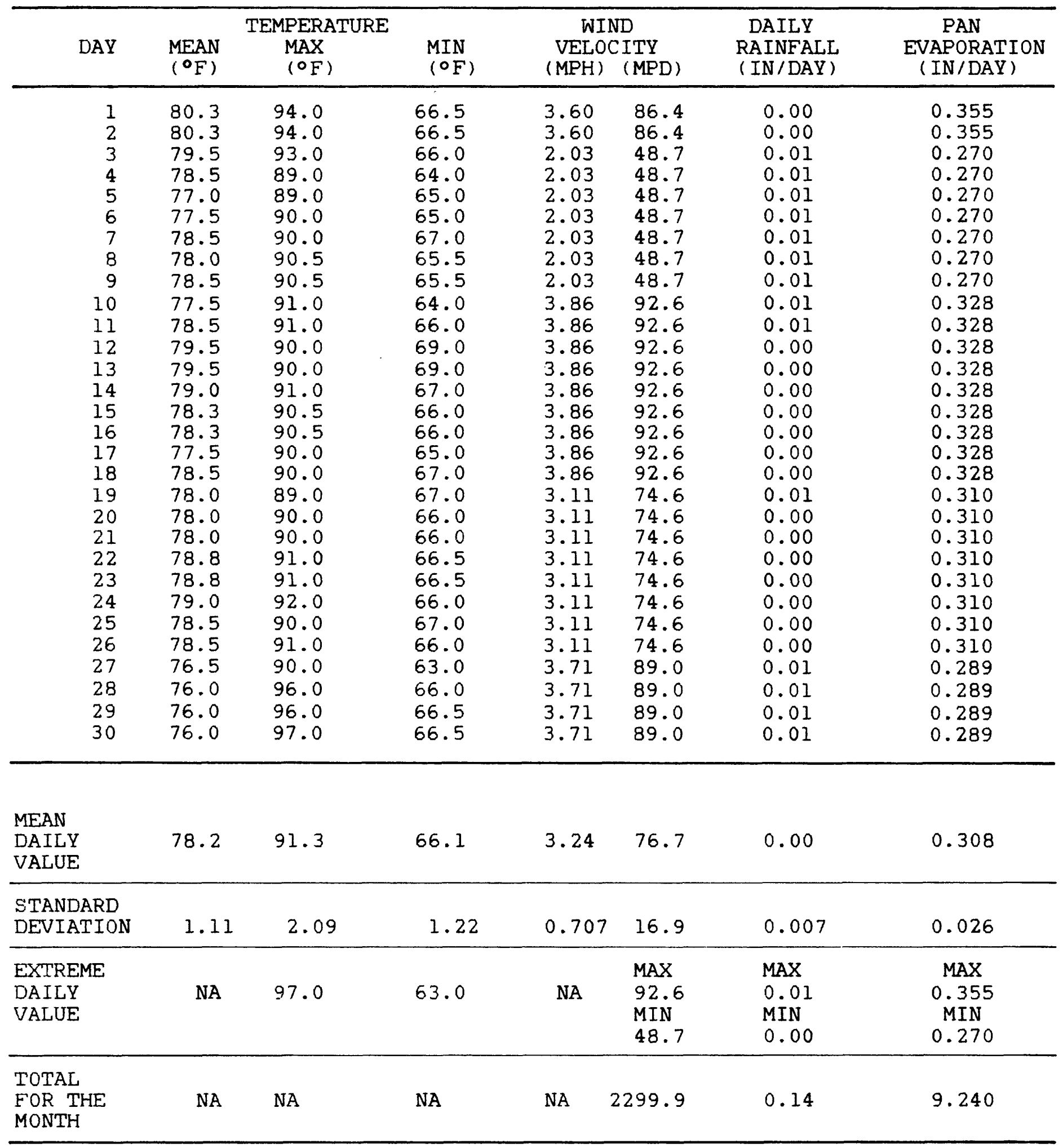




\section{REFERENCES}

Román-Más, A.J., 1985, Water use for rice irrigation: U.S. Geological Survey Progress Report No. 1, 19 p.

Silva, Servando and Vicente-Chandler, José, 1982, Water use by flooded rice in Puerto Rico: The Journal of Agriculture of the University of Puerto Rico, Vol LXVI, no. 3, July 1982, 7 p.

U.S. Department of Commerce, 1970, Weather bureau observing handbook No. 2, substation observations supersedes circular B: Weather Bureau, 1st edition, $77 \mathrm{p}$.

Vicente-Chandler, José, 1977, Cultivo intensidad y perspectivas del arroz en Puerto Rico - Boletín 250: Estación Experimental Agrícola, $71 \mathrm{p}$. 